OUNP-97-10

DESY 97-226

\title{
STRUCTURE FUNCTIONS OF THE NUCLEON AND THEIR INTERPRETATION
}

\author{
A M COOPER-SARKAR \& R C E DEVENISH \\ Physics Department, University of Oxford, \\ Nuclear $\& 3$ Astrophysics Laboratory, \\ Keble Road, Oxford OX1 3RH, England \\ A DE ROECK \\ DESY, \\ Notkestrasse 85, \\ 22607 Hamburg, Germany \\ Presently on leave at CERN Geneva, Switzerland
}

November 1997

Submitted to International Journal of Modern Physics A

\begin{abstract}
The current status of measurements of the nucleon structure functions and their understanding is reviewed. The fixed target experiments E665, CCFR and NMC and the HERA experiments $\mathrm{H} 1$ and ZEUS are discussed in some detail. The extraction of parton momentum distribution functions from global fits is described, with particular attention paid to much improved information on the gluon momentum distribution. The status of $\alpha_{s}$ measurements from deep inelastic data is reviewed. Models and non-perturbative approaches for the parton input distributions are outlined. The impact on the phenomenology of QCD of the data at very low values of the Bjorken $x$ variable is discussed in detail. Recent advances in the understanding of the transition from deep inelastic scattering to photoproduction are summarized. Some brief comments are made on the recent HERA measurements of the ep NC and CC cross-sections at very high $Q^{2}$.
\end{abstract}

\section{Introduction}

There have been two main strands of interest in experiments on deep inelastic scattering (DIS) since the initial observation of Bjorken scaling. Firstly, they are used to investigate the theory of the strong interaction and secondly, they are used to determine the momentum distributions of the partons within the nucleon.

The observation of Bjorken Scaling established that the quark-parton model 
(QPM) is a valid framework in which to interpret the data and thus that the deep inelastic structure functions can be used to measure the nucleon's parton distributions. The later observation of logarithmic scaling violations indicated that the non-Abelian gauge theory of Quantum Chromodynamics (QCD) might be the correct theory of the strong interactions. Parton distributions may still be measured, but one must account for their evolution with $Q^{2}$ a .

In the early 1980's arguments raged, as to whether the observed $Q^{2}$ dependence of structure functions was uniquely described by perturbative QCD (pQCD), or whether alternative theories (e.g. Abelian theories, fixed point theories) provide an equally good description of data. The consensus came down in favour of QCD as more and more accurate data, from a variety of physical processes, were able to establish some of the crucial features of QCD. For example, direct evidence for the existence of spin-1 gluons came from the observation and properties of three jet events in $e^{+} e^{-}$scattering at PETRA. Evidence for the running of the strong coupling constant, $\alpha_{s}$ with $Q^{2}$, came from measurements of production rates for high transverse momentum hadrons and jets, in many different processes at different scales. Evidence for the non-Abelian nature of the gluon-gluon coupling came from the observation that the fraction of the momentum of the nucleon carried by the quarks decreases as $Q^{2}$ increases and from studies of four jet events at LEP.

These observations made the theory of QCD part of the Standard Model (SM) of particle physics, and the variety of different processes which can be successfully described by pQCD is now detailed in many textbooks. Hence the interest in structure function measurements from the mid to late eighties lay in extracting accurate parton distributions taking the framework of pQCD for granted. These parton distributions are of interest since we still have only a limited understanding of the non-perturbative physics involved in confinement. Accurate parton distribution functions are also of vital importance as the input for calculations of high energy scattering processes which might involve physics beyond the Standard Model.

With the advent of DIS data from HERA we have entered into a new phase of interest in structure function measurements, where we are again using the data to investigate the properties of $\mathrm{pQCD}$, in a new kinematic regime where our (by now) conventional calculations may not be adequate. Firstly because the conventional treatment does not account for contributions to the cross-section which are leading in $\alpha_{s} \ln (1 / x)$ and we are now making measurements at very low $x$, and secondly because the parton densities, in particular the gluon, are becoming large and we may need to develop a high density formulation of QCD. It is an appropriate time to take stock of what has been achieved in the five years since HERA started operation in 1992. The first high statistics measurements of $F_{2}^{p}$ have been published by H1 and ZEUS and during the same period the fixed target experiments NMC, E665 and CCFR have also published their complete structure function data sets. Activity on the theoretical side has also increased and there is much to report on, even though we are still some way from a complete understanding of QCD at low $x$. We cover

a $Q^{2}$ and $x$ are defined in Sec. 2.1 
published data up to the end of June 1997 and we include some preliminary data shown at the Symposium on Lepton and Photon Interactions, Hamburg 1997 and at the EPS Conference, Jerusalem 1997日. Material on theoretical interpretations is covered up to the end of November 1997.

This review is concerned with the unpolarized proton and neutron structure functions and their interpretation. It does not deal with the interesting questions raised by DIS data on nuclear targets, such as the EMC effect and nuclear shadowing. We also do not not discuss the field of polarized structure functions and spin dependent effects. It has been established that some $10 \%$ of the cross-section for deep inelastic events consists of events with a significant rapidity gap. These are generally thought to be mediated by diffractive processes discussion of such processes is beyond the scope of the present review, see refs. 3 . for recent review talks. We note that such events are implicitly included in the inclusive measurements from which the structure functions and parton densities are derived. In more detail: section 2 contains a collection of kinematic definitions and standard formulae for deep inelastic neutral-current (NC) and charged-current (CC) scattering cross-sections; section 3 provides an pedagogical outline of the pQCD improved quark-parton model framework; section 4 gives an account of the fixed target and collider detectors relevant to this review and a brief discussion of experimental methods and event reconstruction; section 5 is a review of the structure function data from the NMC, E665, CCFR, H1 and ZEUS experiments; section 6 discusses the extraction of parton distribution functions from this data and the related extraction of the value of $\alpha_{s}$; section 7 provides a broad survey o of theoretical and phenomenological approaches to understanding structure function data at small $x$; section 8 considers the transition region from deep inelastic data to photoproduction: section 9 contains a brief account of the measurements by $\mathrm{H} 1$ and ZEUS of the NC and $\mathrm{CC}$ cross-sections at very large $Q^{2}$ and the observation of a possible excess of events above the Standard Model expectation; finally section 10 contains a summary and outlook.

\section{Formalism}

We give definitions of the commonly used Lorentz invariants and the formulae for the neutral and charged current cross-sections in terms of the structure functions. The latter are put in context by reference to the quark-parton model. The details of how the expressions are derived may be found in many books, for example Halzen and Martin 5 or Roberts 6 .

\subsection{Lorentz invariants}

The basic process is

$$
l N \rightarrow l^{\prime} X
$$

where $l, l^{\prime}$ represent leptons ${ }^{\mathrm{b}}, N$ represents a nucleon and $X$ represents the hadronic

\footnotetext{
$\mathrm{b}$ Lepton is taken to include antileptons, unless it is necessary to distinguish them.
} 
final state particles. Such processes were studied using a lepton beam and a fixed nucleon target until the advent of HERA and the terminology of lepton as probe and nucleon as target is still widely used.

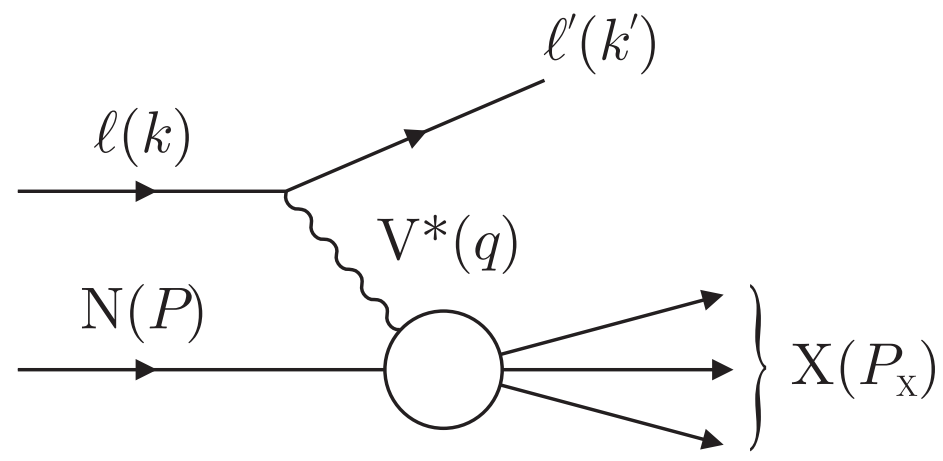

Fig. 1. Schematic diagram of lepton-hadron scattering via Vector-Boson Exchange

The associated four vectors are $k, k^{\prime}$ for the incoming and outgoing leptons respectively, and $P$ for the target (or incoming) nucleon. The process is mediated by the exchange of a virtual vector boson, $V^{*}(\gamma, W$ or $Z)$, see Fig. 1. This boson has four momentum given by

$$
q=k-k^{\prime}
$$

and the four vector $p_{X}$ of the hadronic final state system $X$ is given by

$$
P_{X}=P+q .
$$

Various Lorentz invariants are useful in the description of the kinematics of the process:

$$
s=(P+k)^{2}
$$

the centre of mass energy squared for the $l p$ interaction,

$$
Q^{2}=-q^{2}
$$

the (negative of) the invariant mass squared of the virtual exchanged boson,

$$
x=Q^{2} / 2 P . q
$$

the Bjorken $x$ variable, which is interpreted in the quark-parton model as the fraction of the momentum of the incoming nucleon taken by the struck quark,

$$
W^{2}=(P+q)^{2}
$$

the invariant mass squared of the hadronic system $X$, and

$$
y=P . q / P . k
$$

a measure of the amount of energy transferred between the lepton and the hadron systems.

Note that (ignoring masses),

$$
Q^{2}=s x y, \quad W^{2}=Q^{2}(1 / x-1),
$$


so that only three of these quantities are independent. We shall give formulae appropriate for $Q^{2} \gg M_{N}^{2}$, where $M_{N}$ is the nucleon mass, unless otherwise stated.

\subsection{Neutral current cross-sections}

The general form for the differential cross-section for charged lepton-nucleon scattering, mediated by the neutral current at high energy, is given in terms of three structure functions, $F_{2}, F_{L}, x F_{3}$, as,

$$
\frac{d^{2} \sigma\left(l^{ \pm} N\right)}{d x d Q^{2}}=\frac{2 \pi \alpha^{2}}{Q^{4} x}\left[Y_{+} F_{2}^{l N}\left(x, Q^{2}\right)-y^{2} F_{L}^{l N}\left(x, Q^{2}\right) \mp Y_{-} x F_{3}^{l N}\left(x, Q^{2}\right)\right],
$$

where $Y_{ \pm}=1 \pm(1-y)^{2}$ and we have ignored mass terms as appropriate at high $Q^{2}$. For $Q^{2}$ values much below that of the $Z^{0}$ mass squared, the parity violating structure function $x F_{3}$ is negligible and the structure functions $F_{2}, F_{L}$ are given purely by $\gamma^{*}$ exchange. We begin by considering this region in more detail. The differential cross-section is conveniently rewritten in terms of $R=\sigma_{L} / \sigma_{T}$, the ratio of the longitudinally to transversely polarized virtual photon absorption cross-sections, as follows

$$
\frac{d^{2} \sigma\left(l^{ \pm} N\right)}{d x d Q^{2}}=\frac{4 \pi \alpha^{2}}{Q^{4} x}\left[1-y-\frac{M_{N}^{2} x^{2} y^{2}}{Q^{2}}+\frac{y^{2}}{2} \frac{1+4 M_{N}^{2} x^{2} / Q^{2}}{1+R}\right] F_{2}^{l N}\left(x, Q^{2}\right),
$$

where we have specified the terms in $M_{N}^{2}$ which are important at low $Q^{2}$, and we have used the relationship $R=F_{L} / 2 x F_{1}$, where $2 x F_{1}=F_{2}\left(1+4 M_{N}^{2} x^{2} / Q^{2}\right)-F_{L}$ relates the structure functions $2 x F_{1}, F_{2}$ and $F_{L}$.

It will aid understanding of the significance of the structure functions if we specify their interpretation in terms of the partons within the nucleon as follows:

$$
F_{2}^{l N}\left(x, Q^{2}\right)=\Sigma_{i} e_{i}^{2} *\left(x q_{i}\left(x, Q^{2}\right)+x \bar{q}_{i}\left(x, Q^{2}\right)\right),
$$

a sum over the quark, $x q_{i}$, and antiquark, $x \bar{q}_{i}$, momentum distributions, contained in the nucleon, multiplied by the corresponding quark charge squared $e_{i}^{2}$ (where $e_{i}$ is conventionally understood to be the fraction (quark charge/positron charge)). In writing this formula we have extended the naive quark-parton model interpretation to include $Q^{2}$ dependence following the conventional manner of implementing first order $\mathrm{pQCD}{ }^{\mathrm{c}}$. The spin- $1 / 2$ nature of the quarks also implies that $\sigma_{L}=0$, i.e. there is no longitudinal absorption cross-section for virtual photon scattering on quarks, and thus that $R=0$ and $F_{L}=0$, from which the Callan-Gross relationship 6 , $2 x F_{1}=F_{2}$, follows provided that $Q^{2} \gg 4 M_{N}^{2} x^{2}$. These relationships are also preserved in first order pQCD.

The differential cross-section is then related directly to $F_{2}\left(x, Q^{2}\right)$ by the simple relationship,

$$
\frac{d^{2} \sigma(l N)}{d x d Q^{2}}=\frac{2 \pi \alpha^{2}}{Q^{4} x} Y_{+} F_{2}^{l N}\left(x, Q^{2}\right) .
$$

and thus the lepton-nucleon scattering process has been used extensively to measure quark distribution functions, and to investigate their $Q^{2}$ dependence.

\footnotetext{
${ }^{c}$ At second order the same definition of $F_{2}$ may be used, but only for the DIS renormalization scheme.
} 
Nucleon parton distribution functions (PDFs) are defined to be those for the proton. By default we shall mean the momentum distribution $x q\left(x, Q^{2}\right)$ (also called the momentum density) when we use this term. The number distribution (or density) is given by $q\left(x, Q^{2}\right)$. For ease of reference, we shall specify the flavours entering into the sum for lepton probes on proton, neutron and isoscalar targets. For charged lepton scattering on protons

$$
\begin{aligned}
F_{2}^{l p}\left(x, Q^{2}\right)= & \frac{4}{9} x\left(u\left(x, Q^{2}\right)+\bar{u}\left(x, Q^{2}\right)+c\left(x, Q^{2}\right)+\bar{c}\left(x, Q^{2}\right)\right) \\
& +\frac{1}{9} x\left(d\left(x, Q^{2}\right)+\bar{d}\left(x, Q^{2}\right)+s\left(x, Q^{2}\right)+\bar{s}\left(x, Q^{2}\right)\right)
\end{aligned}
$$

whereas for a neutron target strong isospin swapping gives

$$
\begin{aligned}
F_{2}^{l n}\left(x, Q^{2}\right)= & \frac{4}{9} x\left(d\left(x, Q^{2}\right)+\bar{d}\left(x, Q^{2}\right)+c\left(x, Q^{2}\right)+\bar{c}\left(x, Q^{2}\right)\right) \\
& +\frac{1}{9} x\left(u\left(x, Q^{2}\right)+\bar{u}\left(x, Q^{2}\right)+s\left(x, Q^{2}\right)+\bar{s}\left(x, Q^{2}\right)\right)
\end{aligned}
$$

and an isoscalar target is treated as an average of these two. We have assumed that there is no significant bottom or top quark content in the nucleon. (We note that not all authors define a parton distribution for a heavy quark such as the charm quark. The treatment of heavy quarks is discussed in Sec. 3.6)

At values of $Q^{2}$ comparable to the $M_{Z}^{2}$, the formula for $F_{2}$ must be extended to account for $Z^{0}$ exchange and $\gamma-Z^{0}$ interference as follows

$$
F_{2}^{l N}\left(x, Q^{2}\right)=\Sigma_{i} A_{i}^{L, R}\left(Q^{2}\right) *\left(x q_{i}\left(x, Q^{2}\right)+x \bar{q}_{i}\left(x, Q^{2}\right)\right),
$$

where, for lepton scattering,

$$
A_{i}^{L, R}\left(Q^{2}\right)=e_{i}^{2}-2 e_{i} e_{l}\left(v_{l} \pm a_{l}\right) v_{i} P_{Z}+\left(v_{l} \pm a_{l}\right)^{2}\left(v_{i}^{2}+a_{i}^{2}\right) P_{Z}^{2}
$$

The coupling of the fermions to the currents now depends on whether the polarization of the lepton beam is left $(L)$ or right $(R)$ handed. The notation $e_{l}$ specifies the incoming lepton's charge such that $e_{l}= \pm 1$. The vector and axial-vector couplings of the fermions are given by

$$
v_{f}=\left(T_{3 f}-2 e_{f} \sin ^{2} \theta_{W}\right), \quad a_{f}=T_{3 f}
$$

where the definition holds good for any fermion, whether lepton or quark; $T_{3 f}$ is the weak isospin, and $\theta_{W}$ is the Weinberg angle ${ }^{\mathrm{d}}$. The term $P_{Z}$ accounts for the $Z_{0}$ propagator

$$
P_{Z}=\frac{Q^{2}}{Q^{2}+M_{Z}^{2}} \frac{1}{\sin ^{2} 2 \theta_{W}} .
$$

Furthermore, the parity violating structure function $x F_{3}$ is no longer negligible, and is given by

$$
x F_{3}^{l N}\left(x, Q^{2}\right)=\Sigma_{i} B_{i}^{L, R}\left(Q^{2}\right) *\left(x q_{i}\left(x, Q^{2}\right)-x \bar{q}_{i}\left(x, Q^{2}\right)\right),
$$

$\mathrm{d}$ Neutrinos and charged leptons of the same family form weak isospin doublets with $T_{3}=$ $1 / 2,-1 / 2$ respectively; and the quarks form similar weak isospin doublets, within the families $(u, d),(c, s),(t, b)$, with $T_{3}=1 / 2,-1 / 2$ respectively. 
where, for lepton scattering,

$$
B_{i}^{L, R}\left(Q^{2}\right)=\mp 2 e_{i} e_{l}\left(v_{l} \pm a_{l}\right) a_{i} P_{Z} \pm 2\left(v_{l} \pm a_{l}\right)^{2} v_{i} a_{i} P_{Z}^{2} .
$$

The corresponding cross-sections for antilepton scattering are given by swapping $L \rightarrow R, R \rightarrow L$ in the expressions for $F_{2}$ and $x F_{3}$ given in Eq. 15 and Eq. 20 .

\subsection{Charged current cross-sections}

The charged lepton-nucleon differential cross-sections mediated by the charged current $W^{ \pm}$(where the final state lepton is a neutrino) are given by

$$
\frac{d^{2} \sigma^{C C}\left(l^{ \pm} N\right)}{d x d Q^{2}}=\frac{G_{F}^{2}}{4 \pi x} \frac{M_{W}^{4}}{\left(Q^{2}+M_{W}^{2}\right)^{2}}\left[Y_{+} F_{2}\left(x, Q^{2}\right)-y^{2} F_{L}\left(x, Q^{2}\right) \mp Y_{-} x F_{3}\left(x, Q^{2}\right)\right]
$$

and the correspondence to the neutral current case can be seen easily if we express the Fermi coupling constant $G_{F}$ as

$$
G_{F}=\frac{\pi \alpha}{\sqrt{2} \sin ^{2} \theta_{W} M_{W}^{2}}
$$

Then, using the predictions of first order $\mathrm{pQCD}$, we again have $F_{L}=0$, and the differential cross-section for lepton scattering becomes

$$
\frac{d^{2} \sigma^{C C}\left(l^{-} N\right)}{d x d Q^{2}}=(1-P) \frac{G_{F}^{2}}{2 \pi x} \frac{M_{W}^{4}}{\left(Q^{2}+M_{W}^{2}\right)^{2}} \Sigma_{i}\left[x q_{i}\left(x, Q^{2}\right)+(1-y)^{2} x \bar{q}_{i}\left(x, Q^{2}\right)\right]
$$

whereas for antilepton scattering we have

$$
\frac{d^{2} \sigma^{C C}\left(l^{+} N\right)}{d x d Q^{2}}=(1+P) \frac{G_{F}^{2}}{2 \pi x} \frac{M_{W}^{4}}{\left(Q^{2}+M_{W}^{2}\right)^{2}} \Sigma_{i}\left[(1-y)^{2} x q_{i}\left(x, Q^{2}\right)+x \bar{q}_{i}\left(x, Q^{2}\right)\right]
$$

where the sums contain only the appropriate quarks or antiquarks for the charge of the current and the polarization of the lepton beam, $P=\frac{N_{R}-N_{L}}{N_{R}+N_{L}}$.

We specify the flavours entering into the quark sums for ease of reference. For $l^{-} p \rightarrow \nu X$ and left handed polarization we have

$$
\begin{aligned}
F_{2} & =2 x\left(u\left(x, Q^{2}\right)+c\left(x, Q^{2}\right)+\bar{d}\left(x, Q^{2}\right)+\bar{s}\left(x, Q^{2}\right)\right), \\
x F_{3} & =2 x\left(u\left(x, Q^{2}\right)+c\left(x, Q^{2}\right)-\bar{d}\left(x, Q^{2}\right)-\bar{s}\left(x, Q^{2}\right)\right)
\end{aligned}
$$

and $F_{2}=x F_{3}=0$, for right handed polarization.

Whereas for $l^{+} p \rightarrow \bar{\nu} X$ and right handed polarization we have

$$
\begin{aligned}
F_{2} & =2 x\left(d\left(x, Q^{2}\right)+s\left(x, Q^{2}\right)+\bar{u}\left(x, Q^{2}\right)+\bar{c}\left(x, Q^{2}\right)\right), \\
x F_{3} & =2 x\left(d\left(x, Q^{2}\right)+s\left(x, Q^{2}\right)-\bar{u}\left(x, Q^{2}\right)-\bar{c}\left(x, Q^{2}\right)\right),
\end{aligned}
$$

and $F_{2}=x F_{3}=0$, for left handed polarization. Again we have assumed that there is no significant top or bottom quark content in the nucleon and we have also assumed that we are considering energies above threshold for the production of charmed quarks in the final state ${ }^{\mathrm{e}}$.

e Otherwise we need to multiply $\bar{d}$ by $\cos ^{2} \theta_{c}$ and $\bar{s}$ by $\sin ^{2} \theta_{c}$ in Eq. 26, and $d$ by $\cos ^{2} \theta_{c}$ and $s$ by $\sin ^{2} \theta_{c}$ in Eq. 27 . 
Structure functions ...

For charged lepton scattering on neutron targets these relationships undergo isospin swapping $u \rightarrow d, d \rightarrow u, \bar{u} \rightarrow \bar{d}, \bar{d} \rightarrow \bar{u}$, and if we are considering isoscalar targets we must take the average $(n+p) / 2$.

To date charged current reactions have been studied most extensively in the reverse neutrino and antineutrino scattering processes,

$$
\nu N \rightarrow \mu^{-} X, \quad \bar{\nu} N \rightarrow \mu^{+} X
$$

at $Q^{2}$ values far below the mass of the $W$ squared. The polarizations of the (anti)neutrino probes are automatically (right)left-handed, and these cross-sections have conventionally been written as

$$
\frac{d^{2} \sigma(\nu, \bar{\nu})}{d x d y}=\frac{G_{F}^{2} s}{4 \pi}\left[Y_{+} F_{2}\left(x, Q^{2}\right)-y^{2} F_{L}\left(x, Q^{2}\right) \pm Y_{-} x F_{3}\left(x, Q^{2}\right)\right]
$$

and using the results of first order pQCD we have for neutrino scattering

$$
\frac{d^{2} \sigma(\nu)}{d x d y}=\frac{G_{F}^{2} s}{\pi} \Sigma_{i}\left[x q_{i}\left(x, Q^{2}\right)+(1-y)^{2} x \bar{q}_{i}\left(x, Q^{2}\right)\right]
$$

and for antineutrino scattering,

$$
\frac{d^{2} \sigma(\bar{\nu})}{d x d y}=\frac{G_{F}^{2} s}{\pi} \Sigma_{i}\left[(1-y)^{2} x q_{i}\left(x, Q^{2}\right)+x \bar{q}_{i}\left(x, Q^{2}\right)\right]
$$

Hence antineutrino and neutrino data have been combined to extract quark and antiquark momentum distributions and data from proton and isoscalar targets have been combined to achieve flavour separation.

We specify here the flavours entering into these sums in terms of the structure functions for ease of reference.

$$
\begin{aligned}
F_{2}^{\nu p} & =2 x\left(d\left(x, Q^{2}\right)+s\left(x, Q^{2}\right)+\bar{u}\left(x, Q^{2}\right)+\bar{c}\left(x, Q^{2}\right)\right), \\
x F_{3}^{\nu p} & =2 x\left(d\left(x, Q^{2}\right)+s\left(x, Q^{2}\right)-\bar{u}\left(x, Q^{2}\right)-\bar{c}\left(x, Q^{2}\right)\right), \\
F_{2}^{\nu n} & =2 x\left(u\left(x, Q^{2}\right)+s\left(x, Q^{2}\right)+\bar{d}\left(x, Q^{2}\right)+\bar{c}\left(x, Q^{2}\right)\right), \\
x F_{3}^{\nu n} & =2 x\left(u\left(x, Q^{2}\right)+s\left(x, Q^{2}\right)-\bar{d}\left(x, Q^{2}\right)-\bar{c}\left(x, Q^{2}\right)\right), \\
F_{2}^{\bar{\nu} p} & =2 x\left(u\left(x, Q^{2}\right)+c\left(x, Q^{2}\right)+\bar{d}\left(x, Q^{2}\right)+\bar{s}\left(x, Q^{2}\right)\right), \\
x F_{3}^{\bar{\nu} p} & =2 x\left(u\left(x, Q^{2}\right)+c\left(x, Q^{2}\right)-\bar{d}\left(x, Q^{2}\right)-\bar{s}\left(x, Q^{2}\right)\right), \\
F_{2}^{\bar{\nu} n} & =2 x\left(d\left(x, Q^{2}\right)+c\left(x, Q^{2}\right)+\bar{u}\left(x, Q^{2}\right)+\bar{s}\left(x, Q^{2}\right)\right), \\
x F_{3}^{\bar{\nu} n} & =2 x\left(d\left(x, Q^{2}\right)+c\left(x, Q^{2}\right)-\bar{u}\left(x, Q^{2}\right)-\bar{s}\left(x, Q^{2}\right)\right)
\end{aligned}
$$

Again we are assuming no top or bottom quarks in the nucleon targets and that we are working above threshold for production of charmed quarks in the final state $^{\mathrm{f}}$.

\footnotetext{
${ }^{\mathrm{f}}$ Below the charm threshold, one has to multiply $d$ by $\cos ^{2} \theta_{c}$ and $s$ by $\sin ^{2} \theta_{c}$ in Eq. 32 and $\bar{d}$ by $\cos ^{2} \theta_{c}$ and $\bar{s}$ by $\sin ^{2} \theta_{c}$ in Eq. 34 , where $\theta_{c}$ is the Cabibbo mixing angle. Similar modifications would also be necessary for the neutron reactions.
} 
Having set up the formalism we now proceed to outline the theoretical framework of the pQCD improved quark-parton model.

\section{Theoretical framework}

\subsection{The naive quark-parton model}

The quark-parton model (QPM) grew out of the attempt by Feynman 1 to provide a simple physical picture of the scaling that had been predicted by Bjorken $\mathrm{E}$ and observed in the first high energy deep inelastic electron scattering experiments at SLAC 10 where $F_{2}$ was observed to be independent of $Q^{2}$ for $x$ values around $x \sim 0.3$. The model states that the nucleon is full of point-like non-interacting scattering centres known as partons. The lepton-hadron reaction cross-section is approximated by an incoherent sum of elastic lepton-parton scattering cross-sections, see Fig. 2. In the infinite momentum frame it is then easy to show that the variable $x$ is identified with the fraction of the nucleon's momentum involved in the hard scattering.

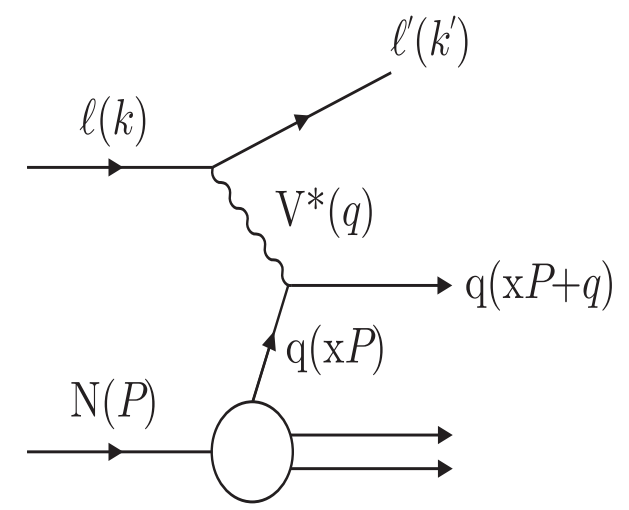

Fig. 2. Schematic diagram of lepton-hadron scattering in the quark-parton model

The parton model had to be reconciled with the static quark model which pictures a nucleon and other baryons as made of three constituent quarks which give them their flavour properties. The reconciliation was effected in the QPM by considering the nucleon as made up of valence quarks, which give it its flavour properties, and a 'sea' of quark antiquark pairs which have no overall flavour. Both the valence quarks and the sea quarks and antiquarks are identified as partons. Hence the antiquark distributions within a nucleon are purely sea distributions, whereas the quark distributions have both valence and sea contributions

$$
x q(x)=x q_{v}(x)+x q_{s}(x), x \bar{q}(x)=x \bar{q}_{s}(x),
$$

and clearly

$$
x q_{s}(x)=x \bar{q}_{s}(x),
$$

To substantiate this idea, early (anti)neutrino data 11 were used to take a first look at the shapes of the sea and valence quark momentum distributions in the 
nucleon. The structure functions for (anti)neutrino scattering on isoscalar targets are given by

and

$$
\begin{aligned}
& F_{2}^{\nu N}(x)=x(u(x)+d(x)+\bar{u}(x)+\bar{d}(x)+2 s(x)+2 \bar{c}(x)) \\
& F_{2}^{\bar{\nu} N}(x)=x(u(x)+d(x)+\bar{u}(x)+\bar{d}(x)+2 \bar{s}(x)+2 c(x))
\end{aligned}
$$

Hence such data may be combined to yield,

$$
\begin{aligned}
& x F_{3}^{\nu N}(x)=x(u(x)+d(x)-\bar{u}(x)-\bar{d}(x)+2 s(x)-2 \bar{c}(x)) \\
& x F_{3}^{\bar{\nu} N}(x)=x(u(x)+d(x)-\bar{u}(x)-\bar{d}(x)-2 \bar{s}(x)+2 c(x))
\end{aligned}
$$

where $x V(x)$ represents a purely valence, or non-singlet, distribution and

$$
F_{2}^{\nu N}(x)=F_{2}^{\bar{\nu} N}(x)=x\left(u_{v}(x)+d_{v}(x)+2 \bar{u}(x)+2 \bar{d}(x)+2 \bar{s}(x)\right)=x V(x)+x S(x)
$$

represents a combination of valence and sea ${ }^{g}$ which is a singlet distribution. The terminology non-singlet/singlet refers to flavour exchange/non-exhange and is explained in context in Sec. 3.2.

Experimental information on valence and sea distributions may also be obtained from charged lepton-nucleon scattering. $F_{2}$ on an isoscalar target may be written as

$$
F_{2}^{l N}=\frac{5}{18} x(u(x)+\bar{u}(x)+d(x)+\bar{d}(x))+\frac{1}{9} x(s(x)+\bar{s}(x))+\frac{4}{9} x(c(x)+\bar{c}(x))
$$

which is a combination of valence and sea quarks and, assuming $\bar{u}(x)=\bar{d}(x)$,

$$
F_{2}^{l p}-F_{2}^{l n}=\frac{1}{3} x\left(u_{v}(x)-d_{v}(x)\right)
$$

is a pure valence distribution. Thus the shapes of the valence and sea distributions may be extracted separately and furthermore, the neutrino and lepton structure functions may be related as follows. Assuming $s(x)=\bar{s}(x), c(x)=\bar{c}(x)$,

$$
F_{2}^{l N}=\frac{5}{18} F_{2}^{\nu N}-\frac{1}{3} x s(x)+\frac{1}{3} x c(x) \approx \frac{5}{18} F_{2}^{\nu N}
$$

The observation that the charged lepton scattering and the neutrino scattering structure functions are related by $\sim 5 / 18$, taken together with the approximate verification of the Callan-Gross relationship was a triumph for the QPM.

Further support came from considering sum rules. Since $x q(x)$ gives the quark momentum distribution, $q(x)$ must give the quark number distribution. Then if we take the quark-parton model seriously, we predict

$$
\int_{0}^{1} d x u_{v}(x)=2, \int_{0}^{1} d x d_{v}(x)=1
$$

Of course these relations cannot be directly verified but their consequences can be, for example the Gross Llewelyn-Smith sum rule 12

$$
\int_{0}^{1} d x F_{3}^{\nu N} \approx \int_{0}^{1} d x\left(u_{v}(x)+d_{v}(x)\right)=3
$$

g In early analyses one often assumed an $\mathrm{SU}(3)$ symmetric sea, i.e. $\bar{u}(x)=\bar{d}(x)=\bar{s}(x), \bar{c}(x)=0$, though it now appears that $\bar{s}(x) \approx 0.5 *(\bar{u}(x)+\bar{d}(x)) / 2$. 
was-rerified in early neutrino data on isoscalar targets. Similarly, the Adler sum rule 13

$$
\int_{0}^{1} \frac{d x}{x}\left(F_{2}^{\bar{\nu} p}-F_{2}^{\nu p}\right)=2 \int_{0}^{1} d x\left(\left(u_{v}(x)-d_{v}(x)\right)=2\right.
$$

was verified using neutrino data on hydrogen targets. The Gottfried sum rule 14

$$
\int_{0}^{1} \frac{d x}{x}\left(F_{2}^{l p}-F_{2}^{l n}\right) \approx 1 / 3 \int_{0}^{1} d x\left(u_{v}(x)-d_{v}(x)\right)=1 / 3
$$

was approximately verified in early data, but more recent data shows that it is actually violated since the approximation in its derivation requires the assumption of an $\mathrm{SU}(2)$ symmetric sea.

A sum rule can also be applied to the sum over the momenta of all types of quarks and antiquarks in the nucleon. Denoting the singlet distribution by

$$
x \Sigma(x)=x(u(x)+\bar{u}(x)+d(x)+\bar{d}(x)+s(x)+\bar{s}(x)+c(x)+\bar{c}(x))=F_{2}^{\nu N}
$$

we have the momentum sum rule (MSR),

$$
\int_{0}^{1} d x x \Sigma(x)=1
$$

if quarks and antiquarks carry all of the momentum of the nucleon. This was not confirmed, the experimental measurement of $\sim 0.5$ implied that there was more momentum in the nucleon than that carried by the quarks and antiquarks and gave impetus to the development of the theory of QCD, in which the deficit in momentum is carried by the gluons.

\subsection{Parton distributions at low and high $x$}

The shapes extracted for valence and sea quark distributions were roughly of the form

$$
x V(x)=A x^{\alpha}(1-x)^{\beta}
$$

where $\alpha \simeq 0.5, \beta \simeq 3$ for the valence distribution, and

$$
x S(x)=B(1-x)^{\gamma}
$$

where $\gamma \simeq 7$ for the sea distribution. Thus the simple picture of a separation between valence and sea quarks contributions was verified 15 .

There are theoretical guidelines for both the low $x$ and the high $x$ behaviour of these distributions. The high $x$ behaviour has been predicted from the dimensional counting rules 16 . As $x \rightarrow 1$ there can be no momentum left for any of the partons apart from the struck quark, thus they become 'spectators' and the prediction is that $x q(x) \rightarrow(1-x)^{2 n_{s}-1}$, where $n_{s}$ is the minimum number of spectators. Thus, for valence quarks one has $n_{s}=2, x V(x) \rightarrow(1-x)^{3}$, and for sea quarks one has $n_{s}=4, x S(x) \rightarrow(1-x)^{7}$, where it may be noted that the counting of spectators represents a very naive view of the interaction. Nevertheless these rules gave a first rough indication of the differing behaviour of valence and sea quarks at high $x^{\mathrm{h}}$.

\footnotetext{
$\mathrm{h}$ For completeness we also note for future reference that for gluons one has $n_{s}=3, x g(x) \rightarrow$ $(1-x)^{5}$.
} 
The low $x$ behaviour is predicted from Regge theory. At small $x$ we have large P. $q$ and hence large centre of mass energy, $W$, of the virtual boson-nucleon system. Thus we are in the Regge region for the virtual boson-nucleon subprocess. Regge phenomenology gives predictions for the scattering amplitudes in high energy hadron-hadron interactions based on considering the process $a b \rightarrow a b$ as mediated by the exchange of a 'trajectory' of virtual particles which have the correct quantum numbers to be exchanged. The prediction is that the imaginary part of the scattering amplitude, $\operatorname{ImA}(a b \rightarrow a b)$, depends on the centre of mass energy of the process squared, $s(a b)$ as (ignoring spin)

$$
\operatorname{Im} A(a b \rightarrow a b) \sim \Sigma_{i} \beta_{i} s(a b)^{\alpha_{i}}
$$

where $\alpha_{i}$ is the intercept of the Regge trajectory and $\beta_{i}$ is an (unknown) residue function.

To gain information on the behaviour of total cross-sections we use the Optical Theorem, which relates the inclusive cross-section to the imaginary part of the forward elastic scattering amplitude as follows

$$
\sigma(a b \rightarrow X)=\frac{1}{s(a b)} \operatorname{Im}(a b \rightarrow a b)
$$

If we apply the Optical Theorem and Regge theory to the virtual boson-nucleon total cross-section (as illustrated in Fig. 3) we predict an $s\left(V^{*} N\right)$ dependence

$$
\sigma\left(V^{*} N \rightarrow X\right) \sim \frac{1}{s\left(V^{*} N\right)} \Sigma_{i} s\left(V^{*} N\right)^{\alpha_{i}} \sim \Sigma_{i} s\left(V^{*} N\right)^{\alpha_{i}-1}
$$

where the sum is dominated by the appropriate trajectory with the largest $\alpha$. This dependence on $s\left(V^{*} N\right)$ translates back to an $x$ dependence of $x^{\left(1-\alpha_{i}\right)}$ for the deep inelastic scattering process. The appropriate Regge trajectory depends
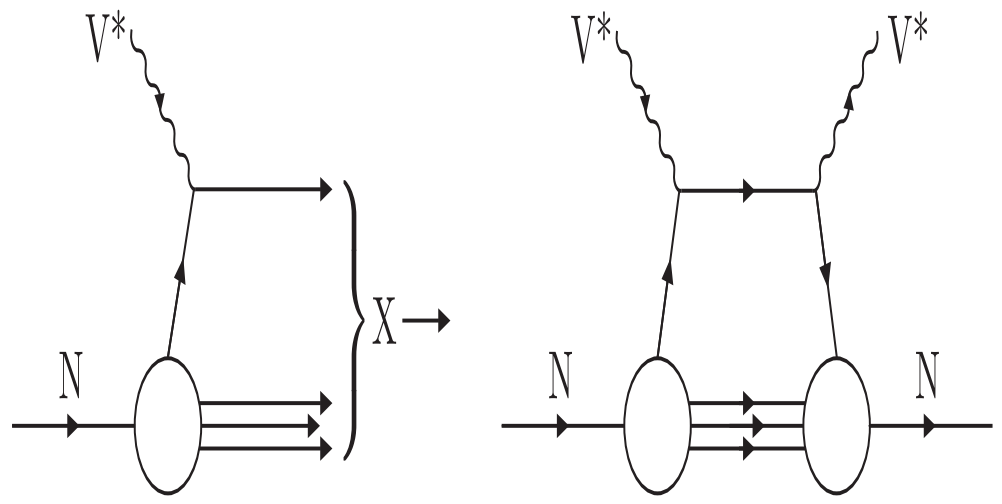

Fig. 3. Illustration of the Optical Theorem

on whether we consider the contribution of $F_{2}$ or $x F_{3}$ to the total cross-section, since these pick out different flavour exchanges. For $\nu, \bar{\nu}$ scattering the exchange in the $\left(V^{*} N \rightarrow V^{*} N\right)$ process has the possible flavour combinations $u \bar{u}+d \bar{d}$ and $u \bar{u}-d \bar{d}$, corresponding to $F_{2}$ and $x F_{3}$ respectively (see Fig. - 4 ). The latter is flavour non-singlet (i.e. flavour IS exchanged) and hence the leading Regge trajectory is 
the $\rho / A_{2}$ trajectory, with intercept $\alpha_{R} \sim 0.5$. The former is flavour singlet and the leading trajectory is called the Pomeron, with intercept $\alpha_{P} \sim 1.0+\lambda$, where $\lambda \sim 0.08$. The exchange of the Pomeron trajectory is considered responsible for the slowly increasing behaviour of hadron-hadron total cross-sections with increasing energy 17 . Hence we have the predictions that the shape of $x F_{3}$ as $x \rightarrow 0$ is given
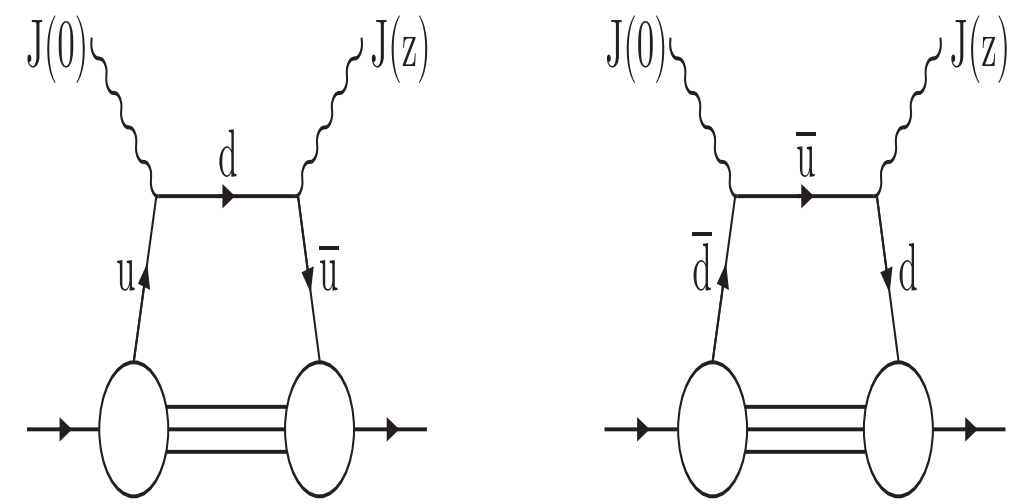

Fig. 4. Flavour structure of $\mathrm{t}$ channel exchanges for the handbag diagram in $\nu, \bar{\nu}$ scattering. The notations $J(z), J(0$ refer to the space-time structure of the vector boson currents.

by $x^{0.5}$, whereas the shape of $F_{2}$ as $x \rightarrow 0$ is given by $x^{-\lambda}$, i.e. it is flattish or very slowly increasing as $x$ decreases. These predictions were born out by the early data, which were mostly taken at moderate $Q^{2}\left(\sim 10 \mathrm{GeV}^{2}\right)$ and $x$ values of around $x \gtrsim 0.01$. It is also true that the real photon nucleon cross-section (for which $Q^{2}=0$ ) obeys the Regge prediction for the Pomeron. However since we now know that parton distributions do not exhibit exact Bjorken Scaling, but evolve with $Q^{2}$, one may ask in what region of $Q^{2}$ should these predictions be relevant? Since Regge phenomenology is an essentially non-perturbative approach we might expect it to be most relevant at low $Q^{2}$. However, the new HERA data allow us to probe very low $x$ values $\left(x \lesssim 10^{-4}\right)$ and we shall see that there are significant deviations from the Regge predictions for Pomeron exchange, even for $Q^{2}$ values as low as $Q^{2} \simeq 2 \mathrm{GeV}^{2}$. This has led to theoretical developments such that the conventional Pomeron referred to above is now called the 'soft' Pomeron to distinguish it from hard Pomeron-like behaviour which can be predicted in the framework of pQCDE. We discuss this further in Secs. 7, 8.

\subsection{Parton distributions with $Q^{2}$ dependence: $Q C D$ evolution}

The QPM must be modified since partons cannot be non-interacting. They are confined within nucleons by the strong interaction. QCD is a non-Abelian gauge theory of the strong interactions between quarks and gluons, which allows us to reconcile short distance freedom with long distance confinement. This comes about because the strong interaction's strength is variable. 


\subsubsection{The running coupling constant}

This is best understood by considering the behaviour of the strong coupling 'constant', $\bar{g}$, which is defined as the value of the $q \bar{q} g$ vertex diagram (see Fig. F) The value of $\bar{g}$ which one measures in strong interactions must include all the virtual

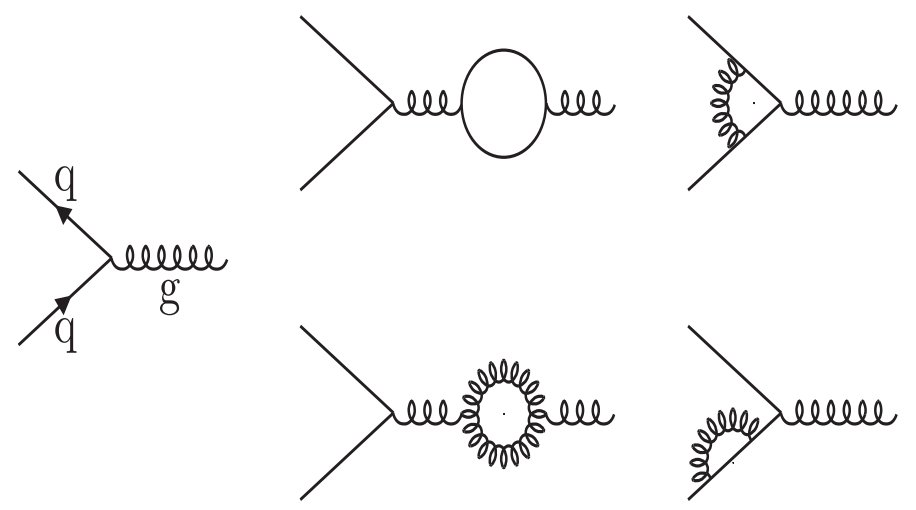

Fig. 5. Schematic diagram of the $q \bar{q} g$ vertex diagram plus virtual loop corrections

loop diagrams (only the 1-loop corrections are illustrated in Fig. F). If one tries to calculate this series of diagrams one obtains infinities and these are controlled by a renormalization procedure, in which one defines the coupling to be finite at some scale $\mu^{2}$, and expresses $\bar{g}\left(Q^{2}\right)$ at any other scale in terms of this fixed value. Renormalization can be performed in various different ways or schemes. It is clear that the results for physical quantities cannot depend on the arbitrary scale $\mu^{2}$ and this independence is expressed in terms of a Renormalization Group Equation, which can be solved to give the dependence of the coupling $\bar{g}$ (or indeed of any Green's Function) on the external scale in terms of a calculable $\beta$ function

$$
2 \frac{d}{d t} \bar{g}(t)=\beta(\bar{g})=-\beta_{0} \frac{\bar{g}^{3}}{16 \pi^{2}}-\beta_{1} \frac{\bar{g}^{5}}{\left(16 \pi^{2}\right)^{2}}+\ldots
$$

where $t=\ln \left(Q^{2} / \mu^{2}\right), \beta_{0}=11-2 n_{i} / 3, \beta_{1}=102-38 n_{i} / 3$, and $n_{i}$ is the number of quarks participating in the interaction at the scale $Q^{2}$. The term in $\beta_{0}$ gives the 1-loop results for the $\beta$ function, the term in $\beta_{1}$ gives the 2-loop result etc. The 1-loop solution of this equation is often expressed in terms of the 'running coupling constant' $\alpha_{s}\left(Q^{2}\right)=\bar{g}^{2}\left(Q^{2}\right) /(4 \pi)$ as

$$
\alpha_{s}\left(Q^{2}\right)=\frac{4 \pi}{\beta_{0} \ln \left(Q^{2} / \Lambda^{2}\right)}
$$

where $\Lambda$ is now a parameter of QCD, which depends on the renormalization scale and scheme and also on the number of active flavours $n_{i}$ at the scale $Q^{2}$ we are working at.

The fact that the coupling constant actually depends on the external scale $Q^{2}$ is true of all field theories including QED, where it manifests itself as charge screening - one does not see the full charge until one probes sufficiently close to the source. However, the non-Abelian nature of the gluon-gluon coupling in QCD leads to antiscreening - the closer one probes the less strong the charge appears!. Thus when 
$Q^{2}$ is fairly large, (say $Q^{2}>4 \mathrm{GeV}^{2}$ for deep inelastic scattering), $\alpha_{s}$ is small and the quarks are 'asymptotically free'. It is in this kinematic region that we may use perturbation theory to perform calculations within QCD. At large distances the coupling constant increases, and quarks are confined within hadrons. We need nonperturbative techniques to work in this region. The present section is concerned only with perturbative QCD, we consider the transition to low $Q^{2}$ in Sec. 8 .

The first order, or 1-loop, expression for $\alpha_{s}$ is not adequate, since the parameter $\Lambda$ cannot be unambiguously defined. Changing the value of $\Lambda, \Lambda \rightarrow \Lambda / k$, changes the expression for $\alpha_{s}$ only by a term of order $\alpha_{s}^{2}$. Since a change in $\Lambda$ is clearly equivalent to a change in the scale $Q^{2}$, we therefore cannot define the scale of the theory at 1-loop. We must compute $\alpha_{s}$ to 2-loops. This is given by

$$
\ln \frac{Q^{2}}{\Lambda^{2}}=\frac{4 \pi}{\beta_{0} \alpha_{s}}-\frac{\beta_{1}}{\beta_{0}^{2}} \ln \left[\frac{4 \pi}{\beta_{0} \alpha_{s}}+\frac{\beta_{1}}{\beta_{0}^{2}}\right]
$$

and a change of scale $\Lambda \rightarrow \Lambda / k$ now produces a change in $\alpha_{s}$ at the same order. Practically, it is also true that we cannot avoid the need to consider higher order effects, since much of the available data is in the moderate $Q^{2}$ range, $5<Q^{2}<$ $100 \mathrm{GeV}^{2}$, where $\alpha_{s}^{2}$ is not negligible.

It is also clear that, when we are working to finite order, $\alpha_{s}$ depends on the renormalization scheme in which we are working, since $\Lambda$ depends on the renormalization scale $\mu$. The choice of scheme was much debated in the early days of pQCD 19,20. One wishes to chose a scheme in which higher order corrections are progressively smaller than the lower order results, i.e. a scheme in which calculations converge rapidly, and at the same time one wishes to chose a scheme which will preserve this property in other perturbatively calculable processes, such as Drell Yan production and $e^{+} e^{-}$scattering). The consensus of the community has settled on the $\overline{\mathrm{MS}}$ scheme although the DIS scheme (which maintains the identification of structure functions in terms of a simple weighted sum over quark distributions) is extensively used in Monte Carlo simulation codes. Values of $\Lambda$ and $\alpha_{s}$ which we quote will refer to the $\overline{\mathrm{MS}}$ scheme unless otherwise stated. We shall indicate how the choice of scheme affects the results as we discuss how pQCD modifies the simple results of the QPM.

\subsection{2. $Q^{2}$ dependence of parton distributions functions: first order}

Firstly, the effect of the quark-gluon interaction is to make the quark momentum distributions, and thus the structure function, depend on $Q^{2}$, (the momentum distributions are said to 'evolve' with $\left.Q^{2}\right)$ such that $F_{2}\left(x, Q^{2}\right)$ rises with $Q^{2}$ at small values of $x$, and falls with $Q^{2}$ at large values of $x$. To understand this $Q^{2}$ evolution we refer to Fig. 6 and Fig. 7. The struck quark may have a history before it interacts with the vector boson. It could radiate a gluon as in Fig. 6 (the QCD Compton (QCDC) process), and thus, although the quark which is struck has momentum fraction $x$, the quark originally had a larger momentum fraction, $y>x$. Alternatively, as in Fig. 7, it may be that a gluon with momentum fraction $y$ produced a $q \bar{q}$ pair and one of these became the struck quark of momentum fraction 


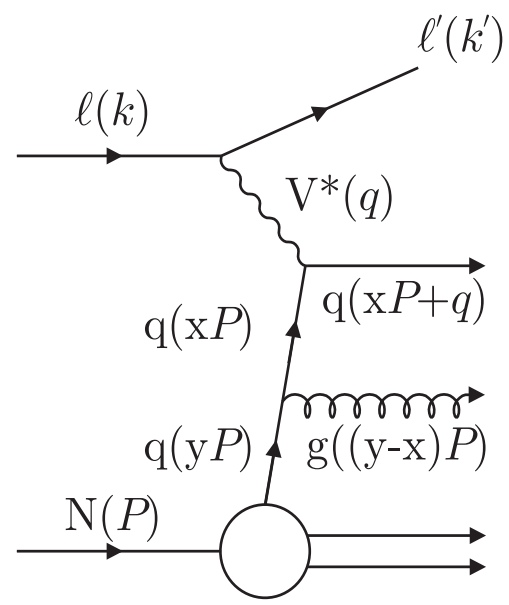

Fig. 6. Schematic diagram of the QCD Compton (QCDC) process

$x$ (the boson-gluon fusion (BGF) process). Thus quark distributions, $q\left(y, Q^{2}\right)$ for all momentum fractions $y$ such that $x<y<1$, contribute to the process shown in Fig. 6, and the gluon distribution $g\left(y, Q^{2}\right)$, for all momentum fractions $y$ such that $x<y<1$, contributes to the process shown in Fig. ㄱ.

To summarize, the parton being probed may not be an 'original' constituent, but may arise from the strong interactions within the nucleon. The smaller the wavelength of the probe (i.e. the larger the scale $Q^{2}$ ) the more of such quantum fluctuations can be observed and hence the amount of $q \bar{q}$ pairs and gluons in the partonic 'sea' increases. Although these sea partons carry only a small fraction of the nucleon momentum, their increasing number leads to a softening of the valence quark distributions as $Q^{2}$ increases. Thus $F_{2}$, which contains both valence and sea quark distributions, will rise with $Q^{2}$ at small $x$, where sea quarks dominate, and fall with $Q^{2}$ at large $x$, where valence quarks dominate

We may quantify these effects using the DGLAP 21 formalism which expresses the evolution of the quark distribution by

$$
\frac{d q_{i}\left(x, Q^{2}\right)}{d \ln Q^{2}}=\frac{\alpha_{s}\left(Q^{2}\right)}{2 \pi} \int_{x}^{1} \frac{d y}{y}\left[\sum_{j} q_{j}\left(y, Q^{2}\right) P_{q_{i} q_{j}}\left(\frac{x}{y}\right)+g\left(y, Q^{2}\right) P_{q_{i} g}\left(\frac{x}{y}\right)\right]
$$

and the corresponding evolution of the gluon distribution (due to contributions from the diagram in which a quark radiates a gluon and from a diagram in which a gluon can split into two gluons) by

$$
\frac{d g\left(x, Q^{2}\right)}{d \ln Q^{2}}=\frac{\alpha_{s}\left(Q^{2}\right)}{2 \pi} \int_{x}^{1} \frac{d y}{y}\left[\sum_{j} q_{j}\left(y, Q^{2}\right) P_{g q_{j}}\left(\frac{x}{y}\right)+g\left(y, Q^{2}\right) P_{g g}\left(\frac{x}{y}\right)\right]
$$

where the 'splitting function' $P_{i j}(z)$ represents the probability of a parton (either quark or gluon) $j$ emitting a parton $i$ with momentum fraction $z$ of that of the parent parton, when the scale changes from $Q^{2}$ to $Q^{2}+d \ln Q^{2}$. These splitting 


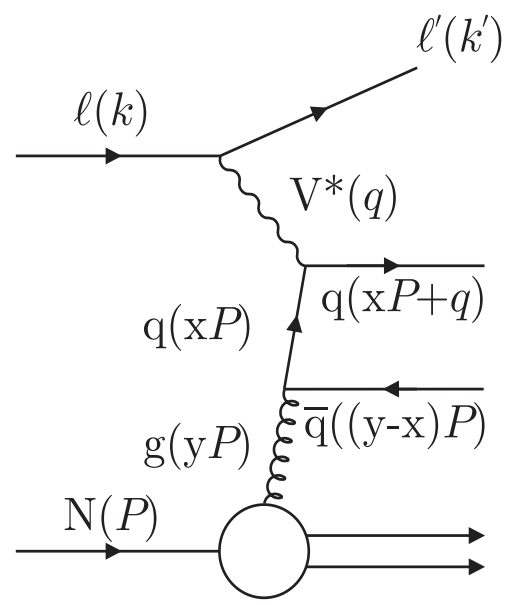

Fig. 7. Schematic diagram of the boson-gluon fusion (BGF) process

functions contribute to the evolution of the parton distributions at order $\alpha_{s}, \alpha_{s}^{2}$, etc.

$$
P_{q q}(z)=P_{q q}^{0}(z)+\frac{\alpha_{s}(t)}{2 \pi} P_{q q}^{1}(z)+\ldots
$$

The diagrams of Fig. 6 and Fig. 17 give the splitting functions which contribute at order $\alpha_{s}$

$$
\begin{gathered}
P_{q q}^{0}(z)=\frac{4}{3} \frac{1+z^{2}}{1-z} \\
P_{q g}^{0}(z)=\frac{1}{2}\left[z^{2}+(1-z)^{2}\right] \\
P_{g q}^{0}(z)=\frac{4}{3} \frac{1+(1-z)^{2}}{z} \\
P_{g g}^{0}(z)=6\left[\frac{z}{1-z}+\frac{1-z}{z}+z(1-z)\right]
\end{gathered}
$$

where the poles at $z=1$ can be regularized by including virtual gluon diagrams, see reference 5 . To this order there is no quark flavour mixing since $P_{q_{i} q_{j}}=0$, unless $i=j$.

Secondly, after we have specified the evolution of the parton distributions we must still relate these parton distributions to the measurable cross-sections and structure functions. Consider, for simplicity, $V^{*} N$ scattering with singlet exchange. For the QPM we may calculate the cross-section in terms of a convolution of the point like $V^{*} q$ scattering (see Fig. 2) and the parton distribution function

$$
\frac{F_{2}(x)}{x}=\int d y d z \delta(x-z y) \sigma^{p o i n t}(z) q(y)
$$

and, in electroproduction for example, we have

$$
\sigma^{\text {point }}(z)=e_{i}^{2} \delta(1-z)
$$

so that

$$
\frac{F_{2}(x)}{x}=e_{i}^{2} q(x)
$$


where clearly one has to sum over all relevant parton flavours to obtain the full result.

Now when this is modified in pQCD it amounts to adding to the pointlike parton cross-section further terms which allow for processes such as $\gamma^{*} q \rightarrow g q$ scattering (see Fig. 6), so that for electroproduction,

$$
\frac{F_{2}(x)}{x}=\int d y d z \delta(x-z y) q(y)\left[e_{i}^{2} \delta(1-z)+\sigma^{\gamma^{*} q \rightarrow g q}\right]
$$

giving,

$$
\frac{F_{2}(x)}{x}=\int_{x}^{1} \frac{d y}{y} q(y)\left[e_{i}^{2} \delta(1-x / y)+\sigma^{\gamma^{*} q \rightarrow g q}\left(\frac{x}{y}, Q^{2}\right)\right]
$$

where

$$
\sigma^{\gamma^{*} q \rightarrow g q}\left(x / y, Q^{2}\right)=e_{i}^{2} \frac{\alpha_{s}}{2 \pi}\left[P_{q q}(z) \ln \frac{Q^{2}}{Q_{0}^{2}}\right]
$$

where $Q_{0}^{2}$ is a low momentum cut-off for the integration pyer the quark propagator which mediates the process $\gamma^{*} q \rightarrow g q$ (see for example 22) and $\alpha_{s}$ is the relevant $q \bar{q} g$ coupling constant. Thus

$$
\frac{F_{2}(x)}{x}=e_{i}^{2} \int_{x}^{1} \frac{d y}{y}\left[q(y)+\Delta q\left(y, Q^{2}\right)\right] \delta\left(1-\frac{x}{y}\right)
$$

so that

$$
\frac{F_{2}(x)}{x}=e_{i}^{2}\left[q(x)+\Delta q\left(x, Q^{2}\right)\right]=e_{i}^{2} q\left(x, Q^{2}\right)
$$

where we have transferred the $Q^{2}$ dependence in the parton cross-section into the parton distribution function $q(x) \rightarrow q\left(x, Q^{2}\right)$. We think of $q\left(x, Q^{2}\right)$ as the effective distribution seen by the vector boson as it explores a wider range of $p_{t}^{2}$ within the nucleon when it has larger $Q^{2}$. We have almost obtained the form of the DGLAP equations since

$$
\Delta q\left(x, Q^{2}\right)=\frac{\alpha_{s}}{2 \pi} \ln \frac{Q^{2}}{Q_{0}^{2}} \int_{x}^{1} \frac{d y}{y} q(y) P_{q q}\left(\frac{x}{y}\right)
$$

can be written as

$$
\frac{d q\left(x, Q^{2}\right)}{d \ln Q^{2}}=\frac{\alpha_{s}}{2 \pi} \int_{x}^{1} \frac{d y}{y} q\left(y, Q^{2}\right) P_{q q}(x / y)
$$

and although this result lacks any contribution from the gluon distribution the extension of the derivation to include the cross-section $\sigma\left(V^{*} g \rightarrow q \bar{q}\right)$ follows similarly. Finally, we have so far treated $\alpha_{s}$ as a constant in the calculation of the cross-section $\sigma\left(V^{*} q \rightarrow g q\right)$, all that remains to complete the DGLAP formalism is to substitute $\alpha_{s} \rightarrow \alpha_{s}\left(Q^{2}\right)$.

\subsection{3. $Q^{2}$ dependence of parton distributions: second order}

First order pQCD introduces $Q^{2}$ dependence into the parton distributions while preserving the simple expressions for the structure functions in terms of the parton distributions. However these expressions require some modification when calculations are made to second order. Such calculations require us to use the 2-loop expression for $\alpha_{s}$ and the $\alpha_{s}^{2}$ contributions from the splitting functions $\left(P_{q q}^{1}(z)\right.$ etc.). The main new feature is that the separation which we made at first order, such that 
the $Q^{2}$ dependence of the parton cross-section was transferred into the parton distribution function, cannot be maintained at second order because it cannot be done in the same way for all processes. Consider the cross-section for $\gamma^{*} q$ scattering at second order. Equivalent to the kernels of Eqs. 72, 73 we now have

$$
e_{i}^{2}\left[\delta(1-z)+\frac{\alpha_{s}}{2 \pi} P_{q q}(z) \ln \frac{Q^{2}}{Q_{0}^{2}}+\alpha_{s} f_{2,3}^{r}(z)\right]
$$

which contains terms $f(z)$ which depend on the symmetry properties of the particular structure function (e.g. $F_{2}, x F_{3}$ ) being considered and on the renormalization scheme in which the calculations are being performed. Hence we should now write

$$
\frac{F_{2}\left(x, Q^{2}\right)}{x}=\int d y d z \delta(x-y z) \sigma\left(z, Q^{2}, \alpha_{s}^{r}\right) q^{r}\left(y, Q^{2}\right)
$$

where the dependence of $\sigma$ and $q$ on the renormalization scheme which defines $\alpha_{s}$ is denoted by the superscript $r$.

The equations which identified the structure functions as sums over quark distributions must be modified accordingly to give expressions like

$$
\frac{F_{2}\left(x, Q^{2}\right)}{x}=\int_{x}^{1} \frac{d y}{y}\left[\sum_{i} C_{2}\left(\frac{x}{y}, \alpha_{s}\right) q_{i}\left(y, Q^{2}\right)+C_{g}\left(\frac{x}{y}, \alpha_{s}\right) g\left(y, Q^{2}\right)\right]
$$

where the sum is over the appropriate flavours of quarks and the coefficient functions $C$ represent the appropriate parts of the $V^{*}$-parton scattering cross-sections,

$$
C_{2}\left(\frac{x}{y}, \alpha_{s}\right)=\sigma_{2}\left(\frac{x}{y}, \alpha_{s}\right)=e_{i}^{2}\left[\delta\left(1-\frac{x}{y}\right)+\alpha_{s}\left(Q^{2}\right) f_{2}\left(\frac{x}{y}\right)\right]
$$

and

$$
C_{g}\left(\frac{x}{y}, \alpha_{s}\right)=\sigma_{g}\left(\frac{x}{y}, \alpha_{s}\right)=\left[\alpha_{s}\left(Q^{2}\right) f_{g}\left(\frac{x}{y}\right)\right]
$$

Similar expressions obtain for $x F_{3}$ in terms of $f_{3}$, but in this case the gluon makes no contribution. One may chose to include the $f_{2}$ term in the definition of the parton distribution so that the coefficient function $C_{2}$ remains as a delta function (this is done in the DIS scheme) but then the coefficient function $C_{3}$ would have to be more complicated. The quark distributions, coefficient functions and splitting functions are all renormalization scheme dependent, and although certain combinations of these quantities are not scheme dependent, in general the predictions for physical quantities such as structure functions ARE renormalization scheme dependent when calculated to finite order. Of course the result summed to all orders cannot depend on the scheme, but we have to live with finite order calculations and thus one must define the scheme in which one is working in order to use the same parton distributions in different physical processes.

One very important consequence of the fact that at second order gluon radiation can no longer be accounted for by making the quark distributions scale dependent, is that we can no longer picture the target purely as a sum of spin $1 / 2$ quarks and thus the Callan-Gross relationship, $2 x F_{1}=F_{2}$, is violated at second order. A 
consequence of this violation is that longitudinal structure function $F_{L}$ is no longer zero. It is given in terms of $F_{2}$ and the gluon distribution as

$$
F_{L}\left(x, Q^{2}\right)=\frac{\alpha_{s}}{\pi}\left[\frac{4}{3} \int_{x}^{1} \frac{d y}{y}\left(\frac{x}{y}\right)^{2} F_{2}\left(y, Q^{2}\right)+2 c \int_{x}^{1} \frac{d y}{y}\left(\frac{x}{y}\right)^{2}\left(1-\frac{x}{y}\right) y g\left(y, Q^{2}\right)\right]
$$

where $c=\sum e_{i}^{2}$ for charged lepton scattering and $c=4$ for neutrino scattering. At small $x\left(x \lesssim 10^{-3}\right)$ the dominant contribution comes from the glue regardless of the exact shape of the gluon distribution. In fact the weighting function in the integral over the gluon distribution approximates to a $\delta$ function 23 , such that a measurement of $F_{L}\left(x, Q^{2}\right)$ is almost a direct measure of the gluon distribution $y g\left(y, Q^{2}\right)$ for $y \simeq 2.5 x$.

\subsubsection{Connection to the moment approach}

The theory of QCD is more formally derived from the Operator Product Expansion and the Renormalization Group Equation to give predictions in terms of the moments of the structure functions. It is not the purpose of the current review to cover this formal approach. Nice expositions are given, for example, in references 196 24. We shall require only some concepts and terminology for our future reference.

The deep inelastic scattering process is calculated by using the Optical Theorem to express it in terms of the elastic process $V^{*} N \rightarrow V^{*} N$. This involves a product of vector boson currents (see Fig. 1). The Operator Product Expansion consists of expanding this current product $J(z) J(0)$ in terms of a sum over operators of different spin and type (e.g. quark or gluon, singlet or non-singlet). For example for the electromagnetic current we have terms such as

$$
\frac{2}{3} \bar{\psi}_{u} \gamma_{\mu} \psi_{u}
$$

So that the product $J(z) J(0)$ contains terms like

$$
\frac{4}{9}\left[\bar{\psi}_{u}(z) \gamma_{\mu} \psi_{u}(z) \bar{\psi}_{u}(0) \gamma_{\mu} \psi_{u}(0)+\ldots\right]
$$

which contract to

$$
\frac{4}{9}\left[\bar{\psi}_{u}(z) \gamma_{\mu} \psi_{u}(0)\right]
$$

which have the form of a sum of coefficients (like $q_{u}^{2}=4 / 9$ ) and operators (like $\bar{\psi} \gamma_{\mu} \psi$ ). These operators can be calculated just like any other Green's Function. Rather than trying to follow the development mathematically we illustrate it schematically in Fig. 8 and Fig. 9 which show how the handbag diagram of Fig. 1 is is expressed in terms of operators, and how this concept is extended when the handbag diagram is modified in $\mathrm{pQCD}$. To obtain the deep inelastic scattering cross-section we must take the nucleon matrix element of the current product, and hence of the operator product expansion. The operator matrix elements are not calculable within perturbative QCD, however their coefficient functions are, and their $Q^{2}$ dependence is given by a renormalization group equation. 


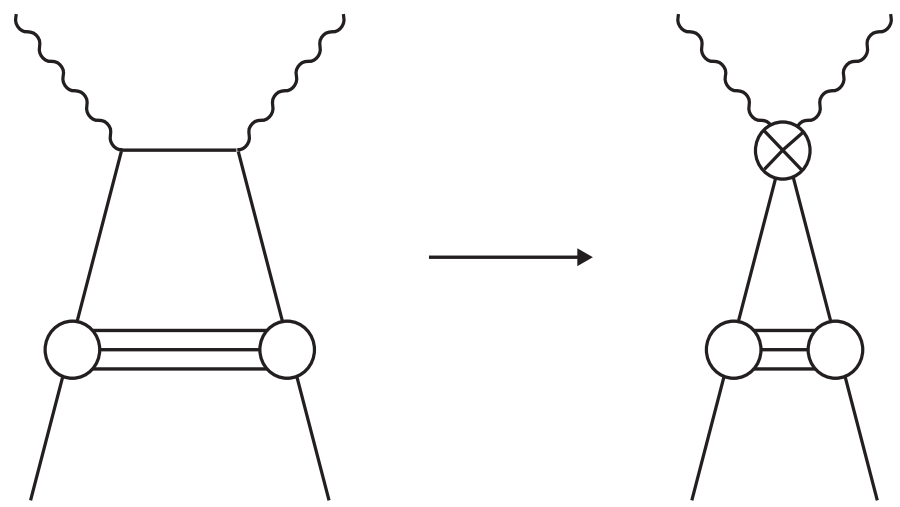

Fig. 8. Schematic diagram of the relationship between the handbag diagram and its operator structure

The results of this formal approach show that the $N$ th order moment of the structure function projects out the spin $N$ contribution to the operator product expansion, e.g. for $F_{2}$

$$
M_{N}\left(Q^{2}\right)=\int_{0}^{1} x^{N-1} \frac{F_{2}\left(x, Q^{2}\right)}{x}=\sum_{a} A_{N}^{a}\left(Q_{0}^{2}\right) C_{N}^{a}\left(\frac{Q^{2}}{Q_{0}^{2}}, \alpha_{s}\right)
$$

where the sum is over the types of operator which can contribute (gluon, (non)singlet quark). The notation $A_{N}^{a}$ represents the operator matrix elements and $C_{N}^{a}$ represents their coefficient functions. In the case when $a$ represents a non-singlet quark operator the $Q^{2}$ dependence of the coefficient function may be written quite simply as

$$
C_{N}^{a}\left(\frac{Q^{2}}{Q_{0}^{2}}, \alpha_{s}\right)=C_{N}^{a}\left(1, \alpha_{s}\right) \exp \left[-\int_{\alpha_{s}\left(Q_{0}^{2}\right)}^{\alpha_{s}\left(Q^{2}\right)} d \alpha \frac{\gamma_{N}^{a}(\alpha)}{\beta(\alpha)}\right]
$$

where $\gamma_{N}^{a}$ is a function known as the anomalous dimension of the non-singlet quark operator, $\beta$ is the QCD $\beta$ function and $Q_{0}^{2}$ is an arbitrary starting point. In the case of singlet quark and gluon operators we have mixing, so that the above equation must be formulated as two coupled equations relating to a 2 by 2 matrix of anomalous dimensions $\gamma^{q q}, \gamma^{q g}, \gamma^{g q}, \gamma^{g g}$.

This formalism may be related to the DGLAP approach as follows. The matrix element $A_{N}^{a}$ of the spin $N$ operator is identified with the $N$ th moment of the corresponding parton distribution $\left(q_{N}^{a}\right)$ evaluated at the same scale $\left(Q_{0}^{2}\right)$ and the $Q^{2}$ dependence of the coefficient function $C_{N}^{a}$ is transferred to the parton distribution such that

$$
q_{N}^{a}\left(Q^{2}\right)=q_{N}^{a}\left(Q_{0}^{2}\right) \exp \left[-\int_{\alpha_{s}\left(Q_{0}^{2}\right)}^{\alpha_{s}\left(Q^{2}\right)} d \alpha \frac{\gamma_{N}^{a}(\alpha)}{\beta(\alpha)}\right]
$$

for non-singlet quarks (and two similar coupled equations are necessary to express the mixing of singlet quarks and gluons). Then Eq. 87 becomes

$$
M_{N}\left(Q^{2}\right)=\sum_{a} q_{N}^{a}\left(Q^{2}\right) C_{N}^{a}\left(1, \alpha_{s}\right)
$$



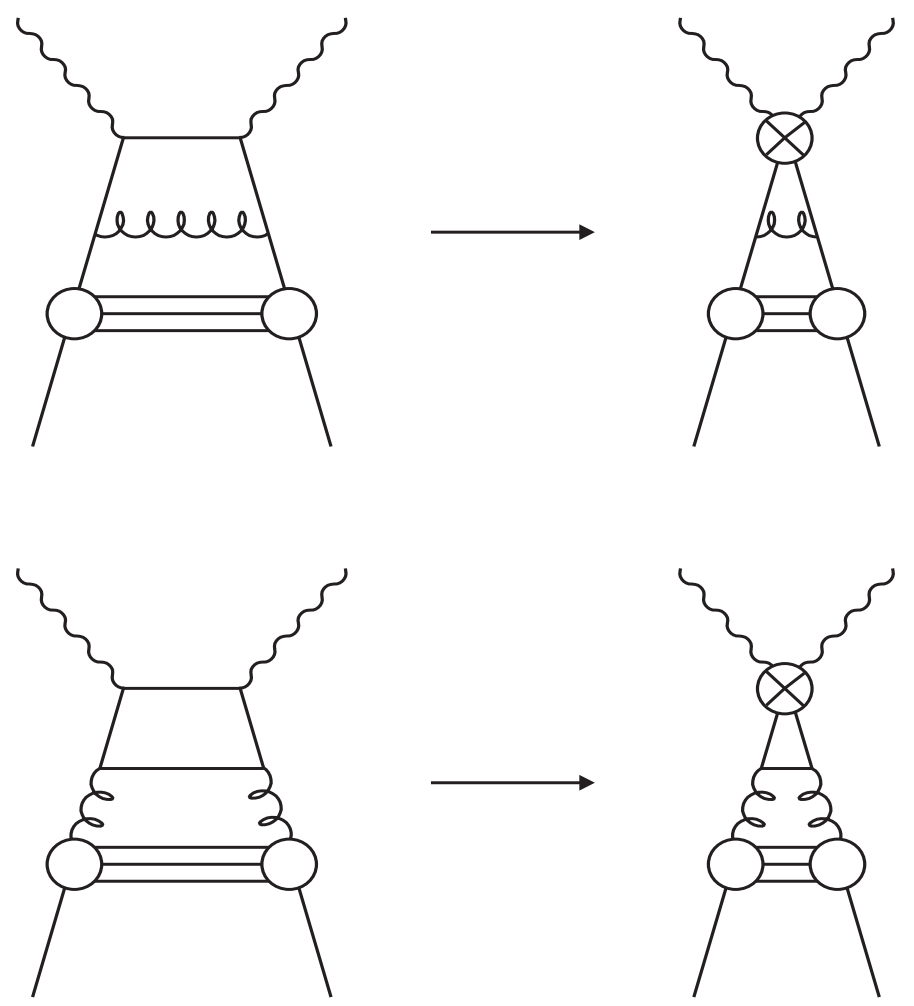

Fig. 9. Schematic diagram of extensions to the handbag diagram from $\mathrm{pQCD}$ and their relationship to quark and gluon operators

and this is precisely what we will get if we take moments on either side of Eq. 80, provided that $C_{N}^{a}$ denotes the moments of the $x$ space coefficient functions and $q_{N}^{a}$ denotes moments of the appropriate type of parton distributions (quark-singlet or gluon). The $Q^{2}$ dependence of the moments of the parton distributions can then be obtained by taking moments of the DGLAP equations

$$
\begin{aligned}
& \frac{d q_{N}\left(Q^{2}\right)}{d \ln Q^{2}}=\frac{\alpha_{s}}{2 \pi}\left[\frac{-\gamma_{N}^{q q}}{4} q_{N}\left(Q^{2}\right)+\frac{-\gamma_{N}^{q g}}{4} g_{N}\left(Q^{2}\right)\right] \\
& \frac{d g_{N}\left(Q^{2}\right)}{d \ln Q^{2}}=\frac{\alpha_{s}}{2 \pi}\left[\frac{-\gamma_{N}^{g q}}{4} q_{N}\left(Q^{2}\right)+\frac{-\gamma_{N}^{g g}}{4} g_{N}\left(Q^{2}\right)\right]
\end{aligned}
$$

where

$$
\frac{-\gamma_{N}^{a}}{4}=\int_{0}^{1} d z z^{N-1} P_{a}(z)
$$

defines the relationship of the anomalous dimension function to the corresponding splitting function.

\subsection{Higher twist}

So far we have only considered the predictions of QCD at leading twist. Twist refers to the (dimension - spin) of the operators entering into the operator product 
expansion. The dominant contributions are twist $=2$ and involve the sort of operators depicted in Fig. 8 and Fig. 9. Higher twist $(\geq 4)$ operators relate to diagrams like those shown in Fig. 10. They are suppressed by powers of $1 / Q^{2}$ in comparison to the leading twist diagrams and so they may become important at low $Q^{2}$, as we approach the region where perturbative QCD becomes inapplicable (since the coupling constant $\alpha_{s}$ becomes too large). In the same kinematic region target mass effects become important. The identification of $x$ with the fraction of the proton's momentum taken by the struck quark canngt be maintained when $Q^{2} \simeq M_{N}^{2}$, and corrections to the formulae are necessary 25. Since these involve powers of $1 / Q^{2}$ they are often called kinematic higher twist effects, whereas terms coming from operators of higher twist are called dynamic higher twist effects.
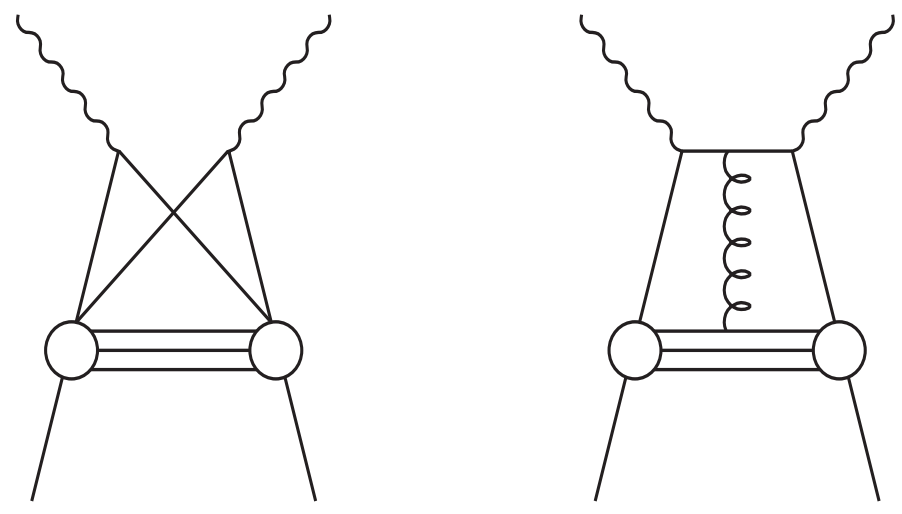

Fig. 10. Alternatives to the handbag diagram for higher twist operators

Conventional higher twist effects are usually only important at higher $x$, for the following reason. At twist $=2$ there are only 2 operators for any value, $N$, of the spin, but at twist $=4$ there are $N$ quark-gluon operators and $N^{2} 4$-quark operators. Hence, because there are at least $N$ times as many operators contributing to the $N$ th moment of the structure function, we expect its $Q^{2}$ dependence to be modified by a multiplicative factor of the form $\left(1+C \frac{N}{Q^{2}}\right)$ and correspondingly the structure function would be modified by a factor of the form $\left(1+C \frac{1}{Q^{2}} \frac{1}{(1-x)}\right)$. Thus such higher twist effects can be important at low $Q^{2}$ and large $x$, i.e. when $W^{2}$ is small and the contributions of specific exclusive processes to the inelastic cross-section become important.

These dynamic higher twist effects have been estimated only for some specific cases 26. Their full calculation awaits a solution to the problem of confinement since they clearly involve reinteraction of the struck quark with the proton remnant. We would then be able to calculate the nucleon matrix elements of all operators and assess their relative contributions in different kinematic regions. Present calculations 27.28 have included contributions to the GLS and Bjorken sum rules and to the longitudinal structure function $F_{L}$. The latter is particularly interesting since only one twist $=4$ operator is involved and hence the higher twist contribution is not 
associated only with high $x$, see ref.29.

There has been some progress on calculating higher twist terms recently, from the renormalon approach 30.31. When calculating physical quantities within QCD the perturbation series will eventually breakdown. Typically such a divergence may come from renormalon graphs - chains of vacuum polarization bubbles on the gluon line. However, the perturbative series which describes such a graph does give a good approximation to the true value if it is truncated appropriately, and one may make an estimate of the error, or ambiguity, on the truncated result. This ambiguity decreases like $Q^{-2 p}$ with increasing $Q^{2}$, where $p$ depends on the particular quantity being calculated. This implies that the perturbative prediction should be supplemented by non-perturbative information from the higher twist contributions. Thus by studying the onset of the breakdown in perturbation theory via renormalon graphs, one gains information on the $Q^{2}$ dependence of the non-perturbative terms which are necessary. This is very useful when studying processes for which one has no handle on such terms from the operator product expansion. Even for DIS where the OPE controls the form of the higher twist terms, this approach may enable us to go further and predict the $x$ dependence of the dominant higher twist contributions. Such predictions give successful descriptions of higher twist contributions to $F_{2}$ and $x F_{3}$ (see Sec. 6.1).

Recently it has been realised that higher twist terms may also be important at very low $x$, this will be considered further in Sec. 7 .

\subsection{The leading log approximation: $L L A, N L L A, D L L A$}

There is a very important aspect which has been glossed over in the preceding subsections. When we make the replacement $\alpha_{s} \rightarrow \alpha_{s}\left(Q^{2}\right)$ to make the DGLAP equations equivalent to the formal theory we are effectively making the virtual boson-parton cross-sections (like $V^{*} q \rightarrow q g$ ) take a $\ln \left(\ln Q^{2}\right)$ dependence rather than a $\ln Q^{2}$ dependence. Thus the contribution of these cross-sections to the parton distribution (which we absorbed into its $Q^{2}$ dependence c.f. Eqs. 73 - 760) is given by

$$
\Delta q\left(x, Q^{2}\right) \sim \alpha_{s} \ln \left(Q^{2}\right)
$$

but from Eq. 59 we know that $\alpha_{s} \sim 1 / \ln Q^{2}$, so the contribution is of order $O(1)$, rather than of order $O\left(\alpha_{s}\right)$. Hence we must go beyond first order and sum terms of the type $\alpha_{s}^{n}\left(\ln Q^{2}\right)^{n}$ (the 'leading logs') to all orders. This is what is actually done by the Renormalization Group Equation in the formal approach and by the DGLAP equations when making the substitution $\alpha_{s} \rightarrow \alpha_{s}\left(Q^{2}\right)$. It is known as the Leading Log Approximation (LLA). The extension to second order includes terms of the type $\alpha_{s}^{n}\left(\ln Q^{2}\right)^{n-1}$ and is known as the Next-to-Leading Log Approximation (NLLA).

The extension of first order results by the LLA is often referred to as leading order (LO) and the extension of second order results by the NLLA is often referred to as next-to-leading order (NLO), however one has to be careful about which quantity is being calculated. An LO result for the structure functions $F_{2}$ or $x F_{3}$ is obtained by using the zeroth order result for the coefficient functions $C_{2}, C_{3}$ 
(i.e. that they are delta functions) together with the order $\alpha_{s}$ contribution from the splitting functions $\left(P_{q q}^{0}\right.$ etc.). An NLO result is obtained by using the first order result for the coefficient functions (see Eqs. 81, 82) together with the order $\alpha_{s}^{2}$ contributions from the splitting functions $\left(P_{q q}^{1}\right.$ etc.). This is what we shall normally mean by the terminologies LO and NLO. However the structure function $F_{L}$, for example, involves coefficient functions which are zero at zeroth order so that the lowest order result for $F_{L}$ involves calculations to the same orders in $\alpha_{s}$ as the NLO result for $F_{2}$. Authors differ as to whether such a result for $F_{L}$ is termed LO or NLO. In this review we shall term such a result lowest order and specify the order of $\alpha_{s}$ involved in the calculation of the relevant coefficient function if ambiguity arises.
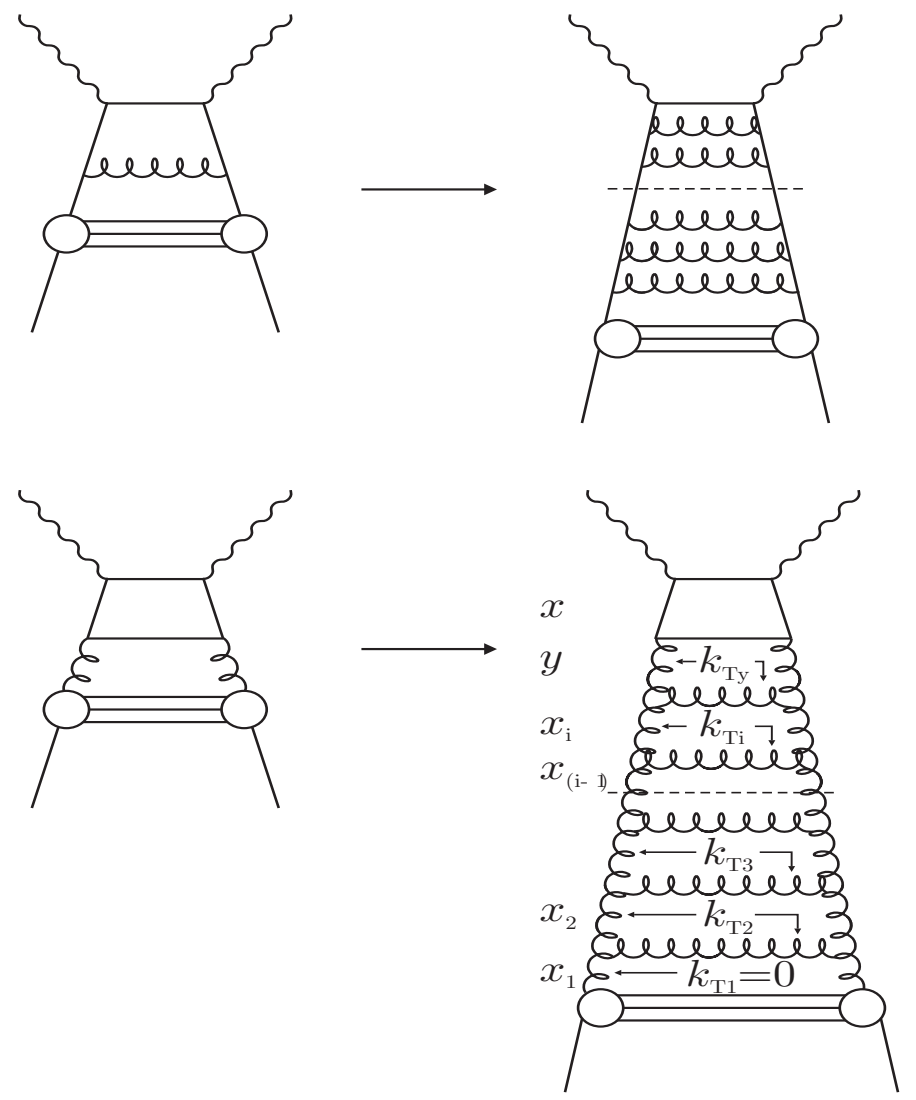

Fig. 11. Schematic representation of ladder diagrams contributing to $V^{*} N \rightarrow V^{*} N$ in the Leading Log Approximation

Diagrammatically we are extending the diagrams of Fig. 9 to include many 'rungs' of gluons as shown in Fig. 11. The dominant contributions to these ladder diagrams come from ordered momenta so that the virtual boson sees successive layers of offmass shell partons all of which contribute to the cross-section. Thus if we label the tchannel partons (struts) from nucleon to boson as 1 to $i$, we have $x_{1}>x_{2} \ldots>x_{i}$ for 
longitudinal momentum fractions and $k_{T, 1}^{2}<k_{T, 2}^{2} \ldots<k_{T, i}^{2}$ for transverse momenta as we go up the ladder.

At large $Q^{2}$ the dominant contribution (LLA) has strong ordering in $k_{T}^{2}, k_{T, 1}^{2}<<$ $k_{T, 2}^{2} \ldots<<k_{T, i}^{2}$, as the ladder diagrams become dominated by collinear gluon emission ${ }^{\mathrm{i}}$. The NLLA contributions correspond to the case when a single pair of gluons are emitted without strong $k_{T}$ ordering, and hence give a power of $\alpha_{s}$ unaccompanied by $\ln Q^{2}$.

We may generalize Eqs. 20, 82 to express collinear factorization

$$
F\left(x, Q^{2}\right)=\int_{x}^{1} \frac{d y}{y} \sigma\left(\frac{x}{y}, \alpha_{s}\left(\mu^{2}\right), \frac{Q^{2}}{\mu^{2}}\right) q\left(y, \mu^{2}\right)
$$

where $F\left(x, Q^{2}\right)$ is a generalized structure function, $q\left(y, \mu^{2}\right)$ is a generalized parton density, and $\sigma\left(z, Q^{2}, \mu^{2}\right)$ is the cross-section for that parton scattering elastically from a virtual boson of virtuality $Q^{2}$. The meaning of the arbitrary scale $\mu$ is illustrated by Fig. 12. It represents the point in the ladder where we chose to sepa-

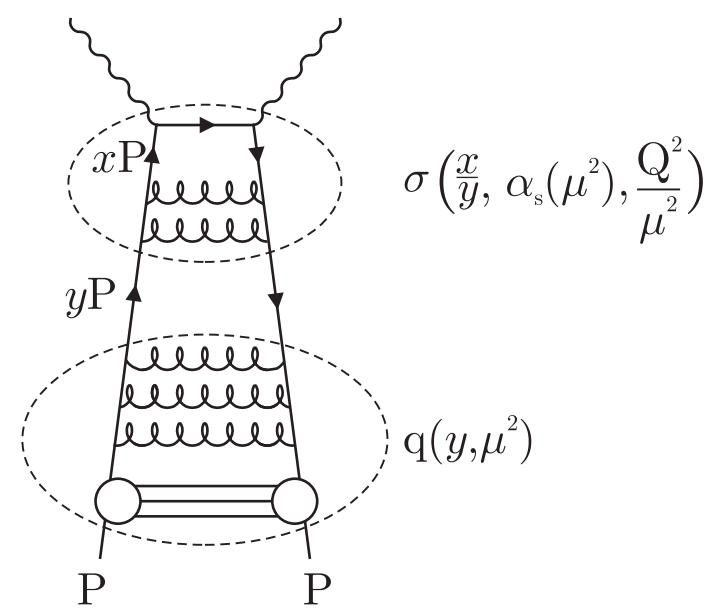

Fig. 12. Schematic representation of collinear factorization in the gluon ladder diagram

rate the parton distributions from their interaction cross-sections. This choice is a part of the renormalization scheme dependence, which is known as the factorization scheme. It essentially defines the point at which we consider that the proton 'ends'. Collinear logarithmic singularities which arise from gluon emission in the partonic subprocess are absorbed into the parton densities (which then run with $Q^{2}$ ). The factorization scheme defines the point at which this happens. So far we have made the choice $\mu^{2}=Q^{2}$, taking the factorization at the top of the gluon chain. Of course the observable structure function must be independent of this choice.

\footnotetext{
$\mathrm{i}$ The first parton of the chain with longitudinal momentum fraction $x_{1}$ is considered to be collinear with the proton so that it has $k_{T, 1}=0$, and the second parton of the chain has $k_{T, 2}$ equal to the $k_{T}$ of the first emitted parton rung. When the ladder becomes dominated by collinear gluon emission one can think of all successive emissions as taking place off partons which are collinear with the proton so that the $k_{T}$ 's of the t-channel gluons (struts) are equal to those of the emitted partons (rungs) as indicated.
} 
At small $x$ and at large $x$ there are large corrections to the conventional leading $\ln Q^{2}$ calculations which imply that $\mu^{2}=Q^{2}$ may not be the best choice of factorization scale. In both cases this arises when there is a second large scale in consideration. At large $x, Q^{2} \gg W^{2}$, but $Q^{2}$ and $W^{2}$ can both still be large. There are large corrections of the form $\alpha_{s}\left(Q^{2}\right) \ln (1-x)$, since as $x \rightarrow 1$ there is less and less phase space for the emission of real gluons $\left(Q^{2}\left(1-x_{i}\right)\right.$ is the upper limit on the invariant mass of the emitted gluon) and thus real gluon emission cannot cancel virtual gluon contributions. It has been suggested that these corrections may be accounted for by replacing $\alpha_{s}\left(Q^{2}\right)$ by $\alpha_{s}\left(Q^{2}(1-x)\right)$ or by $\alpha_{s}\left(W^{2}\right)$. There is interesting new work in this area by Sterman and collaborators 32 . We do not discuss such modifications further since there is little data at very high $x$ on nucleon targets.

At small $x, W^{2} \gg Q^{2}$, but $Q^{2}$ and $W^{2}$ can both still be large. There are large corrections of the form $\alpha_{s}\left(Q^{2}\right) \ln (1 / x)$. In conventional calculations the ordering in $x$ along the gluon ladder becomes strong $x_{1}>>x_{2} \ldots>>x_{i}$, and we then obtain the Double Leading Log Approximation (DLLA) where the cross-sections are dominated by terms in $\ln \left(\ln Q^{2}\right) \ln (1 / x)$. At very low $x$ it may be necessary to go beyond the conventional LLA, NLLA or DLLA and consider terms which are leading in $\ln (1 / x)$ regardless of whether or not they are leading in $\ln Q^{2}$. This is the kinematic region where HERA has probed for the first time and we will consider such extensions to conventional pQCD in detail in Sec. Q7.

\subsection{Heavy quarks}

Firstly we consider the behaviour of $\alpha_{s}$ across flavour thresholds. At second order $\alpha_{s}$ is defined in terms of $\beta_{0}$ and $\beta_{1}$ through Eq. 60. These in turn depend on the number of flavours, $n_{i}$. Thus, for a given value of $\Lambda$, the running coupling $\alpha_{s}\left(Q^{2}\right)$ should reflect the appropriate number of active flavours. For $Q^{2} \ll m_{c}^{2}$ the function $\alpha_{s}\left(Q^{2}\right)$ follows a curve appropriate to 3 -flavours, whereas for $m_{b}^{2} \gg Q^{2} \gg m_{c}^{2}$ it should follow a curve appropriate to 4 -flavours, and for $m_{t}^{2} \gg Q^{2} \gg m_{b}^{2}$ it should follow a curve appropriate to 5-flavours. In the threshold regions, $Q^{2} \sim m_{c}^{2}$ and $Q^{2} \sim m_{b}^{2}$ one must make a smogth transition between different curves. The widely used prescription of Marciano 33 is basically to match the values of $\alpha_{s}$ at the thresholds $Q^{2}=m_{c}^{2}$ and $Q^{2}=m_{b}^{2}$. Explicitly, if one uses $\Lambda$ for 4 flavours as our reference parameter, then defining $\alpha_{s}\left(Q^{2}, n_{i}\right)$ to be the solution of Eq. 60 for $n_{i}$ flavours, we have

$$
\alpha_{s, 4}\left(Q^{2}\right)=\alpha_{s}\left(Q^{2}, 4\right)
$$

for 4 flavours and

$$
\alpha_{s, 5}^{-1}\left(Q^{2}\right)=\alpha_{s}^{-1}\left(Q^{2}, 5\right)+\alpha_{s}^{-1}\left(m_{b}^{2}, 4\right)-\alpha_{s}^{-1}\left(m_{b}^{2}, 5\right)
$$

for 5 flavours and

$$
\alpha_{s, 3}^{-1}\left(Q^{2}\right)=\alpha_{s}^{-1}\left(Q^{2}, 4\right)+\alpha_{s}^{-1}\left(m_{c}^{2}, 4\right)-\alpha_{s}^{-1}\left(m_{c}^{2}, 3\right)
$$

for 3 flavours. One could chose to express $\alpha_{s}$ in terms of $\Lambda$ for 5 flavours or 3 flavours, or one could chose different values for the thresholds, such as $Q^{2}=$ 
$4 m_{c}^{2}, 4 m_{b}^{2}$. It is necessary to specify the procedure being used when comparing results. Values of $\Lambda$ quoted will refer to 4 flavours unless otherwise stated.

Secondly we consider the theoretical issues to be resolved when considering heavy quark production in DIS. These are concerned with the non-trivial question of when can a heavy quark be treated as a massless parton 34 . A range of options has been explored. We consider the contribution of the charmed quark to the structure function, the contributions of bottom and top may be treated similarly. At one extreme charm has been treated as a massless parton. For example, the MRS team 35, 36, 37 assume that $c\left(x, Q^{2}\right)=0$ for $Q^{2} \leq \mu_{c}^{2}$ (where $\mu_{c}=O\left(m_{c}\right)$ ) and generate the charm parton distribution for larger $Q^{2}$ by the splitting $g \rightarrow c \bar{c}$, using the usual NLO DGLAP equations for massless partons. This may be termed a zero mass variable flavour number scheme (ZM-VFN). One has $n_{i}=3+\theta\left(Q^{2}-\mu_{c}^{2}\right)$ and the charm contribution to the structure function is given by $F_{2}^{c \bar{c}}=\frac{8}{9} x c\left(x, Q^{2}\right)$ at LO. The value of $\mu_{c}$ was adjusted to give a satisfactory description of the EMC charmed structure function data. It turns out that the shape of the charmed sea generated by this procedure is similar to that of the non-charmed sea, with normalization given by $2 \bar{c}\left(x, Q_{0}^{2}\right)=\delta S\left(x, Q_{0}^{2}\right), \delta=0.02$ at $Q_{0}^{2}=4 \mathrm{GeV}^{2}$ j.

Obviously, such a procedure cannot give a good description of the charm contribution in the threshold region. In fact one can create a $c \bar{c}$ pair by boson-gluon fusion (BGF), when $W^{2} \geq 4 m_{c}^{2}$, and since $W^{2}=Q^{2}(1-x) / x+M_{N}^{2}$, this can be well below the $Q^{2}$ threshold, $Q^{2} \ll m_{c}^{2}$, at small $x$. Hence, at the other extreme, the GRV team 38390 treat charm as a heavy quark, dynamically generated by the BGF process. This means that there is no concept of a charmed parton distribution we have a fixed flavour number scheme $(\mathrm{FFN})$ with $n_{i}=3$. The contribution to $F_{2}$ comes through BGF as embodied in the DGLAP equations with massive quark coefficient functions. In this picture the lowest order result for $F_{2}^{c \bar{c}}$ arises at order $\alpha_{s}$ in the coefficient functions 41 . However calculations have now been made to next-to-lowest order (order $\alpha_{s}^{2}$ in the coefficient functions) by Laenen et al 42 . $F_{2}^{c \bar{c}}$ is given in terms of the massless parton distributions by

$$
\begin{aligned}
F_{2}^{c \bar{c}}\left(x, Q^{2}\right)= & \frac{Q^{2} \alpha_{s}}{4 \pi^{2} m_{c}^{2}} \int_{a x}^{1} \frac{d y}{y}\left[c_{2, g}^{(0)}+4 \pi \alpha_{s}\left\{c_{2, g}^{(1)}+\bar{c}_{2, g}^{(1)} \ln \frac{\mu^{2}}{m_{c}^{2}}\right\}\right] e_{c}^{2} y g\left(y, \mu^{2}\right) \\
& +\frac{Q^{2} \alpha_{s}^{2}}{\pi m_{c}^{2}} \int_{a x}^{1} \frac{d y}{y}\left[c_{2, q}^{(1)}+\bar{c}_{2, q}^{(1)} \ln \frac{\mu^{2}}{m_{c}^{2}}\right] e_{c}^{2} \sum_{i} y q_{i}\left(y, \mu^{2}\right) \\
& \left.+\frac{Q^{2} \alpha_{s}^{2}}{\pi m_{c}^{2}} \int_{a x}^{1} \frac{d y}{y} d_{2, q}^{(1)} \sum_{i} e_{i}^{2} y q_{i} y, \mu^{2}\right)
\end{aligned}
$$

where $a=1+4 m_{c}^{2} / Q^{2}, e_{i}(i=u, d, s, c)$ are the quark charges in units of the proton charge, $m_{c}$ is the charm quark mass and $\mu$ is a mass factorization scale (which has been put equal to the renormalization scale). The coefficient functions, $c_{2, k}^{(\ell)}, \bar{c}_{2, k}^{(\ell)}, d_{2, k}^{(\ell)}(\ell=0,1 ; k=g, q)$, are calculated in the $\overline{\mathrm{MS}}$ scheme and are functions

$\mathrm{j}$ The latest MRS fits 37 have a starting value of $Q^{2}$ which is less than $\mu_{c}^{2}$ and thus require an additional phenomenological threshold factor to ensure that the charm density turns on smoothly. 
of the variables $\xi=\frac{Q^{2}}{m_{c}^{2}}, \eta=\xi \frac{1-z}{4 z}-1$, as specified in ref. 国. The strong coupling $\alpha_{s}$ is taken as a function of $\mu^{2}$.

In Eq. 99 the terms have been grouped according to their origin. At first order in $\alpha_{s}$ only the gluon distribution function is involved through the coefficient function $c_{2, g}^{(0)}$ (see Fig. 13a). The gluon one loop corrections and bremsstrahlung diagrams (see Fig. 13(b) and (c)) are brought in at order $\alpha_{s}^{2}$ through the coefficient functions

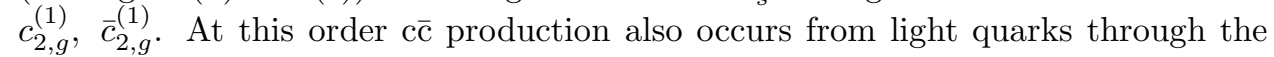

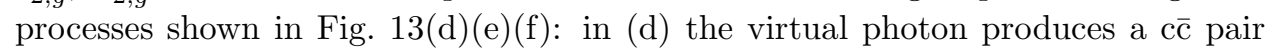
with rate given by the sum of the light quark densities and the coefficient functions $c_{2, q}^{(1)}, \bar{c}_{2, q}^{(1)}$; in (e) and (f) the virtual photon first interacts with a light quark which later produces a c c pair via a gluon with rate given by the charge-weighted sum of the light quark densities and the function $d_{2, q}^{(1)}$.

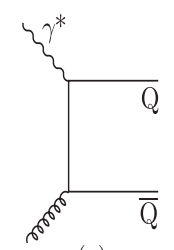

(a)

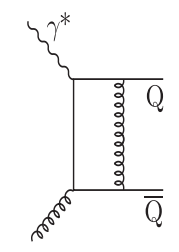

(b)

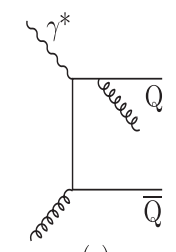

(c)

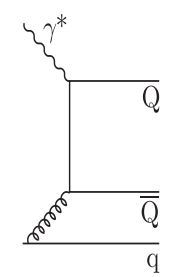

(d)

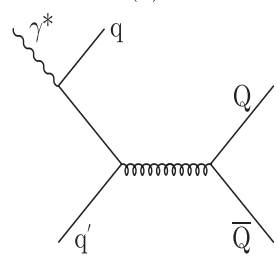

(e)

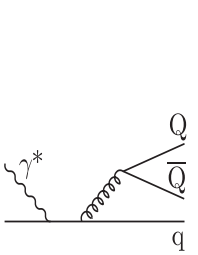

(f)

Fig. 13. The DIS boson-gluon fusion process: (a) order $\alpha_{s}$ diagram; (b) one loop gluon diagram at order $\alpha_{s}^{2}$, there are another 7 similar diagrams plus the one loop quark insertion into the gluon propagator; (c) gluon bremsstrahlung at order $\alpha_{s}^{2}$ - plus another 3 similar diagrams; (d),(e) and (f) are the $\alpha_{s}^{2}$ diagrams which involve the light quarks in the target.

A number of numerical studies of Eq. 99 have been made by the original authors 42 , by Vogt 43 and by Laenen et al 34 for the 1996 HERA workshop. What all these studies show is that $c \bar{c}$ production in DIS is largely determined by the gluon density in the proton and hence is largest at small $x$ - at $Q^{2} \sim 100 \mathrm{GeV}^{2}$ and $x \sim 0.0001$, $F_{2}^{c \bar{c}}$ is more than $30 \%$ of $F_{2}$. The scale dependence of calculations performed to next-to-lowest order is under control: allowing $\mu$ to vary in the range $m_{c} \leq \mu \leq$ $2 \sqrt{Q^{2}+4 m_{c}^{2}}$, gives variations in $F_{2}^{c \bar{c}}$ less than $10 \%$ for $x \leq 0.01$ compared to more than $20 \%$ for calculations made to lowest order. The largest theoretical uncertainty comes from the value of $m_{c}$, a $10 \%$ change in $m_{c}$ produces a $15-25 \%$ change in $F_{2}^{c \bar{c}}$ at a $Q^{2}$ of $10 \mathrm{GeV}^{2}$ (the magnitude of the effect diminishes with increasing $Q^{2}$ ). If a scale of $\mu=\sqrt{Q^{2}+4 m_{c}^{2}}$ is chosen, the gluon is sampled over a rather narrow region in the integral at a fixed $\left(x, Q^{2}\right)$. All this points to $F_{2}^{c \bar{c}}$ being rather a good 
experimental handle on the gluon density. This is discussed further in Sec. 6.1.3.

Fixed flavour number schemes are well suited to the description of differential distributions for charm production just above threshold. As $Q^{2}$ increases into the asymptotic region, $Q^{2} \gg 4 m_{c}^{2}$, one expects that the charmed quark can be treated as a massless parton. However, the coefficient functions of Eq. 99 do not switch smoothly from a heavy quark description to a massless partonic description. The problem is the presence of large logarithms, $\ln \frac{Q^{2}}{m_{c}^{2}}$, in the NLO BGF coefficient functions. In the usual NLO DGLAP calculation of the splitting functions the quarks are taken to be massless and the corresponding large logarithms are summed and absorbed into the definition of the parton distributions (thus giving rise to their scale dependence). If we want to have a consistent description of heavy quark production from the threshold region to the asymptotic region we must find a way to include the heavy quark mass in the DGLAP splitting functions and coefficient functions in such a way as not to destroy the partonic interpretation, so that one can define universal heavy quark distributions which can be used in different processes.

There has progress in this direction recently by Lai and Tung 44 and Martin et al (MRRS) 45. The contribution of charm to $F_{2}$ at NLO is given by a convolution of parton distributions and coefficient functions as

$$
\frac{F_{2}^{c \bar{c}}}{x}=\frac{8}{9} \int_{x}^{1} \frac{d y}{y}\left[C_{c}\left(\frac{x}{y}, Q^{2}, \mu^{2}\right) c\left(y, \mu^{2}\right)+C_{g}\left(\frac{x}{y}, Q^{2}, \mu^{2}\right) g\left(y, \mu^{2}\right)\right]
$$

where $C_{c}=C_{c}^{0}+\frac{\alpha_{s}}{4 \pi} C_{c}^{1}$ and $C_{g}=\frac{\alpha_{s}}{4 \pi} C_{g}^{1}$. In the fixed flavour number scheme which we outlined above one would have $c\left(y, \mu^{2}\right)=0$ by definition and $C_{g}^{1}=C_{g}^{B G F}$ given by the boson-gluon fusion cross-section. Instead of this, the new approaches use general mass variable flavour number schemes (GM-VFN), in which, for $Q^{2} \lesssim m_{c}^{2}$, $c\left(x, Q^{2}\right)=0$ and $F_{2}^{c \bar{c}}$ is described completely by BGF via the $C_{g} \otimes g$ convolution (flavour creation), but for $Q^{2} \geq m_{c}^{2}$ a charm distribution is generated from the $P_{c g}$ splitting $g \rightarrow c \bar{c}$, and an additional contribution to $F_{2}^{c \bar{c}}$ from $\gamma c$ scattering via the $C_{c} \otimes c$ convolution (flavour excitation) results, and rapidly becomes dominant. Once the charm distribution has been created part of the BGF cross-section is automatically generated by the evolution of the charm distribution. To avoid double counting one must subtract from $C_{g}$ the contribution which is generated this way such that, $C_{g}^{1}=C_{g}^{B G F}-\Delta C_{g}$, where $\Delta C_{g} \sim P_{c g}^{0} \otimes C_{c}^{0}$. Thus the troublesome $\ln \left(Q^{2} / m_{c}^{2}\right)$ terms in $C_{g}^{B G F}$ are cancelled by similar terms in $\Delta C_{g}$ and the analysis is applicable from the threshold region into the asymptotic region.

Lai and Tung implement a GM-VFN to LO as first proposed by Aivasiz et al 46 (ACOT), in such a way that the massive terms are incorporated in the coefficient functions so that the charmed partons still evolve like massless partons. There has been recent progress on the NLO implementation by Schmidt 4 and Buza et al th. MRRS chose to calculate massive splitting functions which generate partons which may be used with the conventional $\overline{\mathrm{MS}}$ coefficient functions at NLO. These two approaches are compared by Olness and Scalise 4 . Although the detailed implementations differ, the resulting parton distributions are very similar when evolved to $Q^{2}=25 \mathrm{GeV}^{2}$. There is not yet a consensus on the definitive way to 
implement a GM-VFN scheme. Roberts and Thorne have recently made substantial progress 5 .

\section{The experiments}

In this section we outline the detectors, data selection and extraction of structure functions from the scattering data. We discuss in some detail the NMC, E665 and CCFR fixed target experiments and the two HERA collider experiments H1 and ZEUS.Many excellent reviews cover the earlier deep inelastic experiments from SLAC 10 , FermiLab 51 and CERN 22 that have contributed so much to our understanding of the nucleon structure and strong interactions. The $\left(x, Q^{2}\right)$ regions covered by DIS experiments are shown in Fig. 14 .

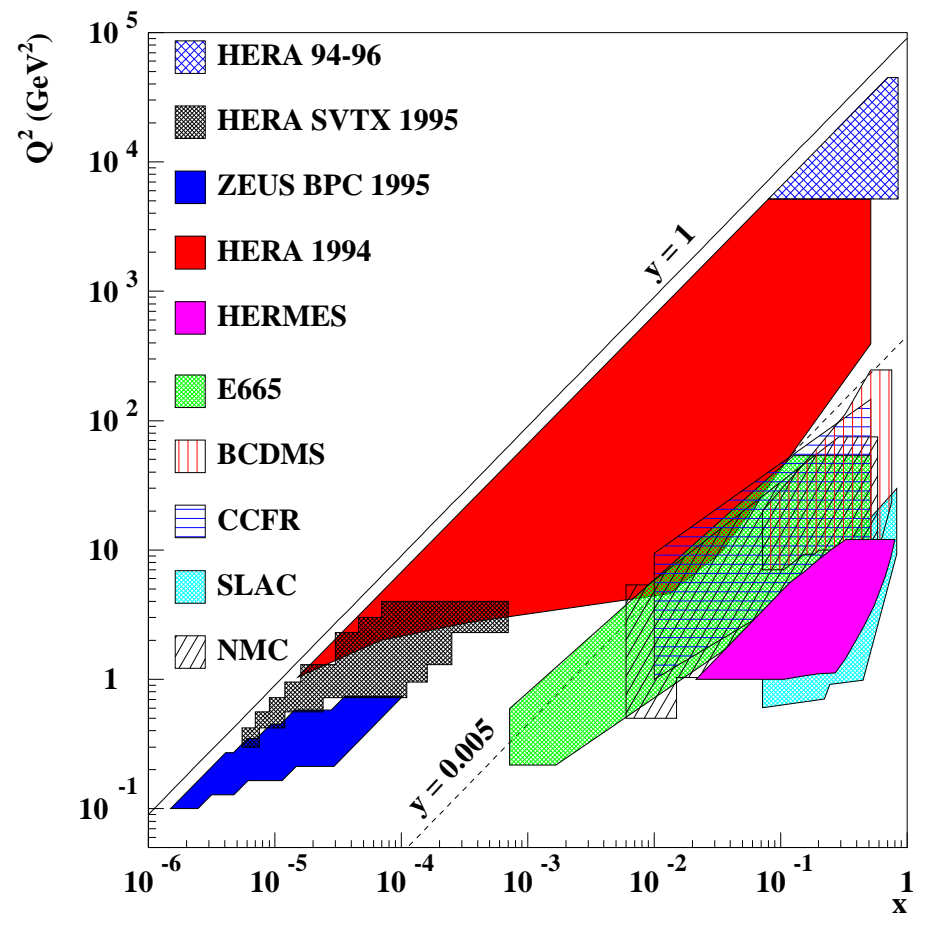

Fig. 14. Regions in the $\left(x, Q^{2}\right)$ plane covered by DIS experiments.

In essence the measurement of the double differential inclusive deep inelastic scattering cross-section is straight forward. Assuming that the kinematic variables are to be reconstructed from the scattered lepton only, one needs precise measurements of the momentum of the beam and scattered leptons, accurate knowledge of the event trigger and reconstruction efficiencies, accurate knowledge of the detector acceptance, good control of backgrounds and radiative corrections and a precise measurement of the luminosity. As a number of the corrections, particularly the radiative corrections, depend on the structure functions, an iterative procedure is 
used to extract $F_{i}\left(x, Q^{2}\right)$ from the raw data. Although simple in principle, to get systematic errors below $5 \%$ requires a very thorough knowledge of the detector and careful attention to many details during both data-taking and analysis. As we shall see below there are quite considerable differences in the problems faced by the fixed target and collider experiments.

\subsection{Fixed target experiments}

First we outline the determination of the variables $x$ and $Q^{2}$ from the measurement of the scattered lepton in fixed target experiments. Assume that the beam energy, $E$, is known and that the lepton beam direction defines the axis from which the scattered lepton angle, $\theta$, is measured. $E^{\prime}$ is the energy of the scattered lepton and $M_{N}$ is the nucleon mass. Then, ignoring the lepton masses,

$$
\begin{gathered}
s=2 M_{N} E \quad \text { and } \quad y=\left(E-E^{\prime}\right) / E, \\
Q^{2}=4 E E^{\prime} \sin ^{2} \frac{\theta}{2}, \\
x=\frac{2 E E^{\prime} \sin ^{2} \frac{\theta}{2}}{M_{N}\left(E-E^{\prime}\right)},
\end{gathered}
$$

definitions of the Lorentz invariants $x, y, s$ and $Q^{2}$ are given in Sec. 2.1. It is also useful to define the energy transfer in the lab frame $\nu=E-E^{\prime}$. In the $\left(x, Q^{2}\right)$ plane lines of constant scattering angle $\theta$ are given by $Q^{2}=s x D /(x+D)$ where $D=2 E \sin ^{2} \frac{\theta}{2} / M_{N}$ and lines of constant $E^{\prime}$ are given by straight lines $Q^{2}=2 M_{N}\left(E-E^{\prime}\right) x$. The need for a well reconstructed scattered lepton requires minimum values of $\theta$ and $E^{\prime}$ which then limit the region in which data can be analysed. Further restrictions also follow from how the resolutions of $x$ and $Q^{2}$ depend on the resolutions of $\theta$ and $E^{\prime}$. This can be seen be looking at the contributions to the relative errors

$$
\begin{aligned}
\frac{\delta Q^{2}}{Q^{2}} & =\frac{\delta E^{\prime}}{E^{\prime}} \oplus \cot \left(\frac{\theta}{2}\right) \delta \theta \\
\frac{\delta x}{x} & =\frac{1}{y} \frac{\delta E^{\prime}}{E^{\prime}} \oplus \cot \left(\frac{\theta}{2}\right) \delta \theta
\end{aligned}
$$

where $\oplus$ indicates addition in quadrature. From these equations we see that $Q^{2}$ is well determined except at very small scattering angles. The same is also true of $x$, but in addition, the error in the energy gets magnified at small values of $y$. Looking at Eq. 103 one sees that errors in the beam energy $(E)$ will also get magnified at small $y$. For fixed target experiments accurate control of $\delta E, \delta E^{\prime}$ and the relative calibration of $E$ and $E^{\prime}$ are very important. Together the restrictions on resolution and acceptance give the regions for the fixed target experiments in the $\left(x, Q^{2}\right)$ plane that are shown in the lower right corner of Fig. 14.

\subsection{1. $N M C$}

The New Muon Collaboration (NMC) experiment 53 at CERN was an extension and improvement of the European Muon Collaboration (EMC) experiment. Originally 
designed to provide better data on nuclear effects in DIS, particularly the EMC effect, it has also provided accurate data on $F_{2}^{p}$ and $F_{2}^{d}$. The NMC experiment was situated on the M2 beam line of the CERN SPS and took data at muon energies of 90, 120, 200 and $280 \mathrm{GeV}$ at various times during 1986, 1987 and 1989. A schematic diagram of the NMC apparatus is shown in Fig. 15.

The important features of the NMC detector for structure function measurements are the following:

BMS. The beam momentum station measures the deviation of beam muons from a central assumed momentum. It achieves an rms resolution of about $1 \mathrm{GeV}$ at $280 \mathrm{GeV}$.

FSM. The forward spectrometer magnet, associated upstream proportional chambers and large area downstream drift chambers provide accurate track reconstruction and momentum determination for charged particles with a typical momentum resolutions of $\Delta p / p \approx 10^{-4}$. Scattered muons are identified by large area drift chambers placed after the electromagnetic calorimeter and a $2 \mathrm{~m}$ thick steel absorber.

BCS. The beam calibration spectrometer provides absolute calibration of beam muons for the BMS. It is situated $36 \mathrm{~m}$ behind the FSM. Special runs at 90, 200 and $280 \mathrm{GeV}$ gave $0.2 \%$ accuracy.

Targets. The target setup has been designed to reduce systematic effects, particularly for cross-section ratio measurements. For the $p$ and $d$ runs it consisted of two similar pairs of $3 \mathrm{~m}$ long target cells exposed alternately to the beam. In one pair the upstream target was liquid hydrogen and the downstream target liquid deuterium, while in the other pair the order was reversed. The acceptance of the main spectrometer is significantly different for the upstream and downstream target positions, giving two determinations of the structure functions for each material.

Trigger. Various triggers can be constructed by using signals from the trigger hodoscopes in the forward spectrometer and from the beam halo veto system. Two physics triggers were used:

T1. The large angle physics trigger. This requires a scattered muon pointing at the target with a minimum scattering angle of $10 \mathrm{mrad}$ together with suppression of events with large energy transfer $\nu$ at small scattering angles (almost real photoproduction).

T2. The small angle trigger. This overlaps with $\mathrm{T} 1$, but uses some additional small hodoscopes to cover small scattering angles in the range $5-15 \mathrm{mrad}$.

Many other triggers were used for beam and beam halo studies and for beam normalization.

As we have noted above, it is very important to have accurate absolute and cross calibrations of the beam and forward spectrometers. This was achieved in a series of dedicated runs at all beam energies with specially installed silicon microstrip 

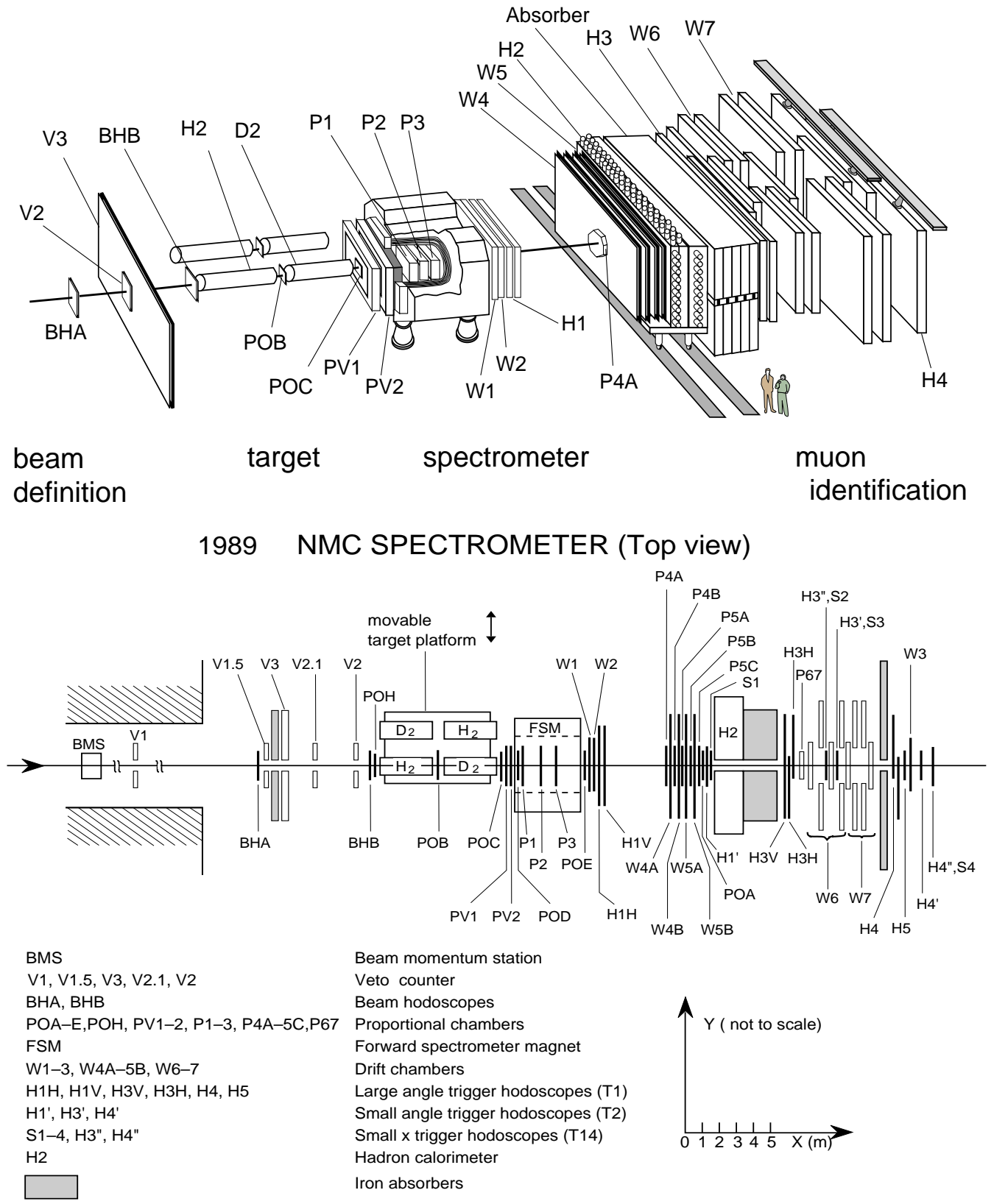

Fig. 15. The NMC detector. 
Table 1. Cuts applied to the NMC data, as explained in the text. Different values of $\theta_{\text {min }}$ were used for the upstream and downstream targets, as indicated. $N_{p}$ and $N_{d}$ are the total number of events for protons and deuterons, respectively, after applying all cuts.

\begin{tabular}{cccccccccc}
\hline Trigger & $\begin{array}{c}E_{\mu} \\
{[\mathrm{GeV}]}\end{array}$ & $\begin{array}{c}p_{\mu}(\min ) \\
{[\mathrm{GeV}]}\end{array}$ & $\begin{array}{c}\nu_{\min } \\
{[\mathrm{GeV}]}\end{array}$ & $\begin{array}{c}\theta_{\min }^{\text {up }}[\mathrm{mrad}] \\
\text { T1 }\end{array}$ & $\begin{array}{c}\theta_{\min }^{\text {down }} \\
{[\mathrm{mrad}]}\end{array}$ & $y_{\max }$ & $\begin{array}{c}W_{\max }^{2} \\
{\left[\mathrm{GeV}^{2}\right]}\end{array}$ & $\begin{array}{c}N_{p} \\
{\left[10^{3}\right]}\end{array}$ & $\begin{array}{c}N_{d} \\
{\left[10^{3}\right]}\end{array}$ \\
\hline & 120 & 20 & 7 & 13 & 15 & 0.9 & 130 & 255 & 533 \\
& 200 & 35 & 20 & 13 & 15 & 0.9 & 150 & 103 & 215 \\
& 280 & 40 & 30 & 13 & 15 & 0.9 & 250 & 56 & 114 \\
& $E_{\mu}$ & $p_{\mu}(\min )$ & $\nu_{\min }$ & $\theta_{\min }^{\text {up }}$ & $\theta_{\min }^{\text {down }}$ & $y_{\max }$ & $y_{\max }$ & $N_{p}$ & $N_{d}$ \\
\hline $\mathrm{T} 2$ & 200 & 35 & 15 & $6-6.5$ & $6.5-7$ & - & 0.8 & 78 & 162 \\
& 280 & 40 & 30 & $6-7$ & $6-7.5$ & 0.2 & 0.8 & 97 & 207 \\
\hline
\end{tabular}

detectors. The calibration of the FSM was also checked to an accuracy of $0.2 \%$ against the measured masses of $K^{0}$ mesons at 90 and $120 \mathrm{GeV}$ and $J / \psi$ mesons at 200 and $280 \mathrm{GeV}$.

The integrated incident muon flux was measured by random sampling of the beam 54, and by reconstructing beam tracks using prescaled triggers on two planes of the beam hodoscope. The second method gave a statistical precision of $1 \%$ in a few hours of data taking.

Selection cuts were applied to the data according to the trigger and the muon beam energy, $E_{\mu}$ as given in Table 1. The scattered muon momentum, $p_{\mu}$, was required to be above a minimum value to suppress muons from pion and kaon decays. Events with small $\nu$, where the spectrometer resolution is poor, were rejected. Regions with rapidly varying acceptance were excluded by requiring minimum muon scattering angles, $\theta_{\text {min }}$. Cuts on the maximum values of $y$ and the mass squared of the hadronic final state, $W^{2}$, excluded the kinematic domain where higher order electroweak processes dominate. The position of the reconstructed vertex was constrained to be within one of the targets. Finally, at each value of $x$, data in regions of $Q^{2}$ where the acceptance was less than $30 \%$ of the maximum at that $x$ were removed.

\subsection{2. $E 665$}

The Experiment E665 5 was located at the end of the NM beamline at Fermilab. The NM beam provided muons of average energy $470 \mathrm{GeV}$ with a spread of $50 \mathrm{GeV}$. The experiment took data in 1987-88, 1990 and 1991-92. The detector, shown in Fig. 16, was designed to measure beam and scattered muons with high precision and to provide a good measurement of charged and neutral particles in the final state. Components important for structure function measurement are:

Beam spectrometer. This was positioned between the end of the NM beamline and the main detector. It consisted of 4 measuring stations each equipped with beam hodoscopes and multiwire proportional chambers, two before a dipole magnet (NMRE) and two after, resulting in a resolution on the beam momentum of 


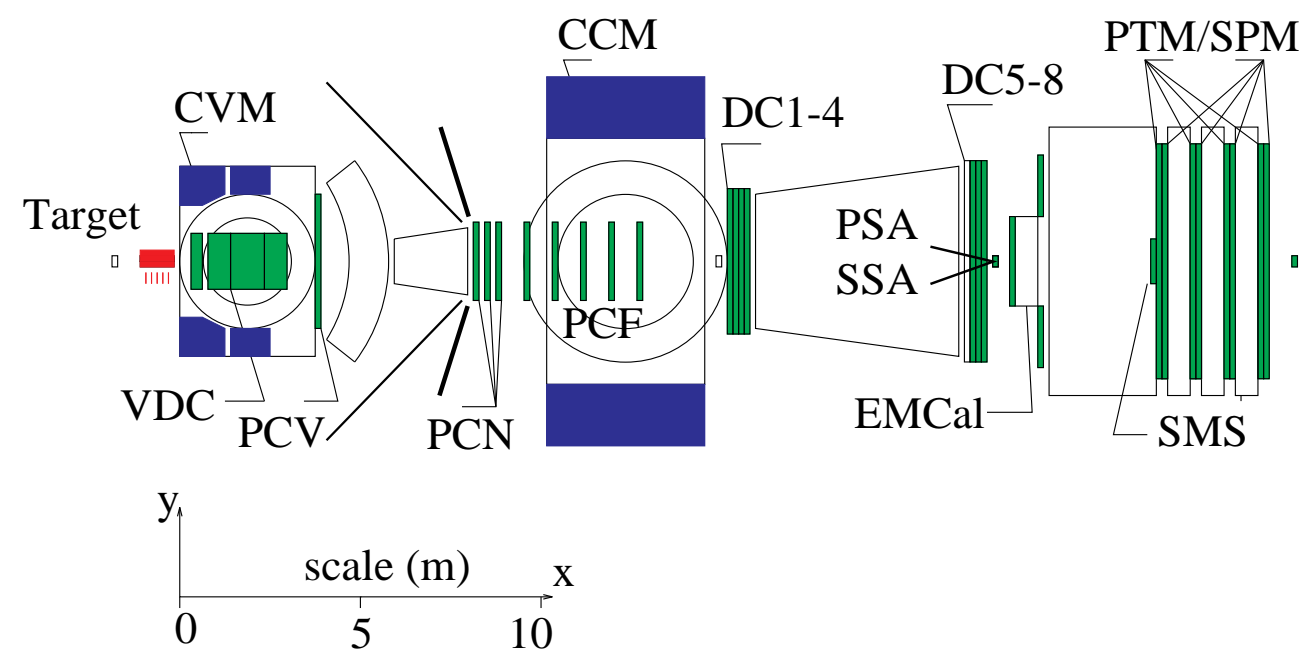

Fig. 16. The E665 detector.

$\delta\left(p^{-1}\right) \sim 8 \times 10^{-6} \mathrm{GeV}^{-1}$.

Target. The target assembly was placed in the field free region in front of the first spectrometer magnet (CVM). It consisted of three identical target cells on a precision table that moved the targets laterally into the beam following a regular cycle. The three target cells were identical and of active length $1 \mathrm{~m}$, two were filled with liquid hydrogen and liquid deuterium respectively. The third was empty and was used to provide data for subtraction of off-target scatters.

Main spectrometer. The charged particle spectrometer was constructed around two large magnets with reversed polarities positioned so that the position of the scattered muon at a focussing plane (where the muon detector was situated) was independent of momentum and depended only on the scattering angle. Tracking was performed by wire planes placed inside both magnets and before and after the CCM. For tracks that traversed the full length of the spectrometer a momentum resolution of $\delta\left(p^{-1}\right) \sim 2 \times 10^{-5} \mathrm{GeV}^{-1}$ was achieved, which gave resolutions of about $5 \%$ on $x$ at low $x$ and about $4 \%$ on $Q^{2}$. Muons were identified by four sets of wire planes placed behind a $3 \mathrm{~m}$ iron absorber, with the sets separated by $1 \mathrm{~m}$ thick concrete absorbers.

EMcal. The electromagnetic calorimeter was placed just in front of the muon absorber and was a 20 radiation length lead gas-sampling device. In addition to measuring photons from neutral hadron decays it also provided information on elastic muon-electron scatters.

Trigger. Various triggers were used in the experiment, for beam normalization, for detector and beam monitoring and for physics. Three classes of physics triggers were used: 1) calorimeter - which used calorimeter signals to identify muon 
interaction events without using signals from behind the absorber; 2) large-angle which required a muon to be identified in the muon chambers behind the absorber; 3) small-angle (SAT) - which used only the veto hodoscope to indicate the absence of an unscattered muon, and allowed events with muon scattering angles as small as $1 \mathrm{mrad}$ to be triggered.

The integrated incident muon flux was measured by a variation on the EMC technique 54. It assumes that the beam spectrometer response is the same for random and physics triggers. The number of usable beam muons is determined by counting the number of random beam triggers with a good muon and multiplying by the pre-scale factor. The latter is determined by comparing the number of random beam triggers with the actual number counted by scalers.

The calibration and resolution of the E665 spectrometers has been checked using the following techniques. Primary protons at $800.6 \pm 2 \mathrm{GeV}$ (determined from the Tevatron magnet currents) were directed through the beam and main spectrometers. The forward spectrometer measured $800.5 \pm 0.14 \mathrm{GeV}$ and an error estimate of $0.3 \%$ was found for the momentum calibration of the beam spectrometer. The relative calibration of the beam and main spectrometers was checked using noninteracting muons and comparing the difference of the momenta measured in the two spectrometers in data and simulation. This lead to an estimate of $0.13 \%$ relative error at the nominal muon beam energy of $470 \mathrm{GeV}$. Elastic muon-electron scattering events also provided information on the calibration and resolution of the spectrometer. Using signals from the EMcal, the events have a distinctive signature and are constrained by kinematics to have $x=m_{e} / M_{N}$. From the measured $x$ distribution one finds the absolute value correct to $0.1 \%$ with a resolution $\delta x / x$ of $5.5 \%$. Finally an uncertainty of $0.35 \%$ in the momentum calibration of the forward spectrometer was estimated from $K_{S}^{0}$ decays.

Using only the small-angle trigger, $159,853 \mathrm{H}_{2}, 100,648 \mathrm{D}_{2}$ and 31,796 empty target events were collected with beam muon energy in the range $350-600 \mathrm{GeV}$. For the structure function sample further cuts were applied: a single scattered muon from within the target with a minimum momentum of $100 \mathrm{GeV}$; a muon energy loss, $\nu$, of at least $35 \mathrm{GeV}$ with calculated relative error less than $50 \%$.

\subsubsection{Neutrino scattering experiments}

Two high statistics neutrino scattering experiments were performed in the 1980s, CDHSW at CERN and CCFR at Fermilab. Both have been important for providing tests of $\mathrm{pQCD}$ and for helping to untangle parton distributions. More details on these and earlier neutrino experiments are given in the reviews by Fisk and Sciulli11 and Mishra and Sciulli 1 . However, because the greatest precision has been achieved by CCFR their results for $F_{2}^{\nu N}$ and $x F_{3}^{\nu N}$ are the ones that continue to be used in global parton determinations. We give a brief account of the CCFR detector.

The CCFR neutrino scattering data were collected in two Fermilab experiments, E740 in 1984 and E770 in 1987. The detector was exposed to the Tevatron Quad-Triplet wide-band neutrino beam, which was composed of neutrinos 


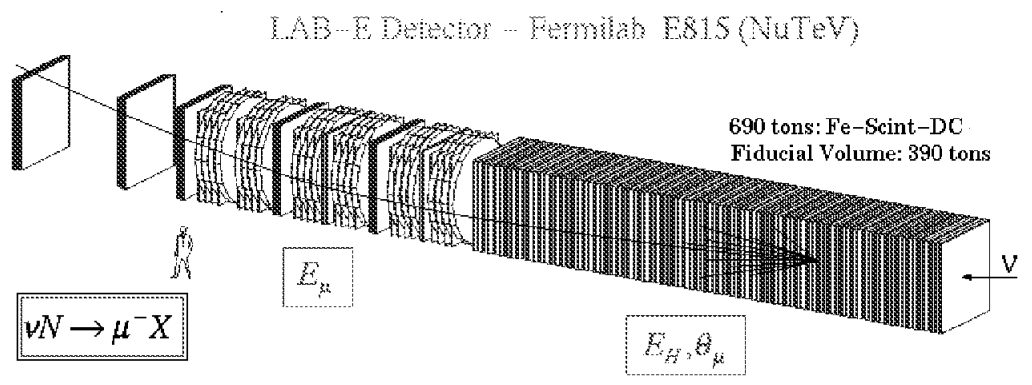

Fig. 17. The CCFR detector.

with average energy $185 \mathrm{GeV}$ and antineutrinos of average energy $143 \mathrm{GeV}$. The maximum beam energy was about $600 \mathrm{GeV}$ and the ratio of $\nu: \bar{\nu}$ was about 2.5 : 1 . The CCFR detector $5 \mathrm{~g}^{\mathrm{k}}$ consists of a $17.7 \mathrm{~m}$ long 690 ton unmagnetized steel-scintillator target calorimeter, which is instrumented with drift chambers for muon tracking. The hadronic energy resolution of the calorimeter is $\sigma / E=0.85 / \sqrt{E(\mathrm{GeV})}$. The calorimeter energy scale was calibrated to $1 \%$ using momentum analysed hadron beams with energies between 15 and $450 \mathrm{GeV}$. The target is followed by a $17.8 \mathrm{~m}$ long solid iron toroidal magnetic spectrometer for muon identification and momentum measurement. The spectrometer was calibrated to about $0.5 \%$ using a momentum analysed muon beam with energies of 50,75, 120 and $200 \mathrm{GeV}$. The muon momentum resolution is $\Delta p / p=0.11$ and it is limited by multiple Coulomb scattering in the iron. The detector provides measurements of the visible hadronic energy $E_{\text {had }}$, the momentum, $p_{\mu}$, and angle with respect to the neutrino beam line, $\theta_{\mu}$, of the scattered muon. The relative neutrino flux at different energies and the relative $\bar{\nu} / \nu$ flux are obtained from events with low hadron energy, $E_{\text {had }}<20 \mathrm{GeV}$. The absolute normalization is determined so that the total $\nu N$ cross-section equals the average value for Fe from the CHDSW and CCFR experiments of $\sigma^{\nu N} / E=(0.677 \pm 0.014) \times 10^{-42} \mathrm{~m}^{2} \mathrm{GeV}^{-1}$ per nucleon 57 .

In neutrino scattering, the incident neutrino energy cannot be measured directly but has to be inferred from the final state measurements. The kinematic variables $x$ and $Q^{2}$ are first estimated from the measured quantities by:

$$
Q_{v i s}^{2}=4 E_{v i s} E_{\mu} \sin ^{2} \frac{\theta_{\mu}}{2}, \quad x_{v i s}=\frac{Q_{v i s}^{2}}{2 M_{N} E_{\text {had }}},
$$

since the lepton energy loss $\nu_{v i s}=E_{\text {had }}$. Average values of physical quantities are

\footnotetext{
$\mathrm{k}$ The detector is also the basis of that for the $\mathrm{NuTeV}$ experiment.
} 
calculated by using Monte Carlo simulation to correct distributions of the corresponding visible quantity.

The data sample consists of 950,000 $\nu$ and 170,000 $\bar{\nu}$ events after the fiducial and kinematic cuts: $p_{\mu}>15 \mathrm{GeV} ; \theta_{\mu}<0.15 \mathrm{rad} ; E_{\text {had }}>10 \mathrm{GeV}$ and $30<E_{v i s}<$ $360 \mathrm{GeV}$. The cuts are designed to select the region of high efficiency and small systematic errors.

\subsection{HERA Experiments}

HERA is the first $e p$ collider and consists of two separate rings of circumference $6.3 \mathrm{~km}$, one a warm magnet electron (or positron) ring with maximum energy $30 \mathrm{GeV}$ and the other a superconducting magnet proton ring of maximum energy $820 \mathrm{GeV}$. The rings are brought together at four intersection regions now occupied by the experiments H1, ZEUS, HERA-B (proton fixed wire target B physics) and HERMES (polarized-electron - polarized nuclear gas-jet target). Although not optimized for the task, HERMES can in principle also measure unpolarized structure functions in a similar kinematic region to the SLAC experiment.

HERA can operate with up to 220 bunches in each ring. In 1994 (1995) the collider operated with 153 (174) colliding bunches of $27.5 \mathrm{GeV}$ positrons and $820 \mathrm{GeV}$ protons. Additional 15 (6) positron and 17 (15) proton bunches were used to study beam related backgrounds. Data were collected in 1992 and 1993, but we shall concentrate on the high statistics measurements from 1994 and some results from the 1995 data.

The interval between bunch crossings in HERA is 96 ns which necessitates sophisticated multi-level trigger systems. For ZEUS and H1, at the first level data is stored temporarily ('pipelined') while hardware specific trigger processors, operating synchronously with HERA beam crossings, arrive at a decision in about $2-5 \mu \mathrm{s}$. The higher trigger levels operate asynchronously and involve more sophisticated calculations, culminating in an 'event filter' which uses a fast version of the offline reconstruction code running on a farm of RISC processors. The overall reduction achieved is from a raw interaction rate of many $100 \mathrm{KHz}$ (which is very sensitive to beam conditions in HERA) to about $5-10 \mathrm{~Hz}$ written to tape.

The H1 and ZEUS detectors are nearly hermetic multi-purpose devices designed to investigate all aspects of high energy ep collisions. In particular both the scattered electron and the hadronic system in a hard $e p$ interaction are measured, the latter allowing one to estimate the energy and angle of the struck quark. Together with the information from the scattered electron one has an overdetermined system and there are 6 possible ways 8 to reconstruct $x$ and $Q^{2}$. We will only discuss those that have been used in $F_{2}$ analyses.

First the electron (E) method. The proton axis is conventionally taken to define the positive $z$ direction from which all angles are measured. At low $Q^{2}$ this means that the electron scattering angle approaches $180^{\circ}$. If $E_{e}, E_{p}$ are the electron and proton beam energies, $E^{\prime}$ and $\theta_{e}$ the energy and angle of the scattered electron, 
then:

$$
\begin{gathered}
s=4 E_{e} E_{p} \quad \text { and } \quad y_{e}=\left(1-\frac{E^{\prime}}{E_{e}} \sin ^{2} \frac{\theta_{e}}{2}\right), \\
Q_{e}^{2}=4 E_{e} E^{\prime} \cos ^{2} \frac{\theta_{e}}{2}=\frac{E^{\prime 2} \sin ^{2} \theta_{e}}{1-y_{e}}, \\
x_{e}=\frac{E_{e} E^{\prime}\left(1-\sin ^{2} \frac{\theta_{e}}{2}\right)}{E_{p}\left(E_{e}-E^{\prime} \sin ^{2} \frac{\theta_{e}}{2}\right)} .
\end{gathered}
$$

Note that if $E^{\prime}=E_{e}$ then $x=x_{0} \equiv E_{e} / E_{p}$, the position of the so-called kinematic peak. In the $\left(x, Q^{2}\right)$ plane lines of constant scattering angle $\theta$ are given by $Q^{2}=s x C /(s x+C)$ where $C=4 E_{2}^{2} \sin ^{2} \frac{\theta_{e}}{2}$ and lines of constant $E^{\prime}$ are given by $Q^{2}=\left(4 x E_{e}\left(E_{e}-E^{\prime}\right)\right) /\left(x_{0}-x\right)$. Fig. 18 shows contours of constant energy of the scattered lepton. The large region of phase space around the kinematic peak, in which $E^{\prime} \approx E_{e}$, is evident. Kinematic peak events, which can be selected to be almost independent of the structure function samples, are used for calibration purposes by both $\mathrm{H} 1$ and ZEUS. A typical selection is shown by the shaded region in the figure. As in fixed target experiments, $E^{\prime}$ is required to be greater than a minimum value and $\theta_{e}$ less than a maximum angle if it is to be measured in the rear EM calorimeters of the collider detectors. These considerations limit the accessible region of $x$ and $Q^{2}$. Although there are differences of detail, the formulae for the resolutions of $x$ and $Q^{2}$ are similar to those of Eq. 104 and the message is the same, that at small $y$ (large $x$ ) the resolution in $x$ deteriorates. The other potential problem with the electron method is that radiative corrections can be large.

In the double angle (DA) method, only angles are used to reconstruct $x$ and $Q^{2}$. In addition to $\theta_{e}$ one constructs the angle $\gamma$ from the hadronic energy flow using:

$$
\cos \gamma=\frac{\left(\sum_{h} p_{x}\right)^{2}+\left(\sum_{h} p_{y}\right)^{2}-\left(\sum_{h}\left(E-p_{z}\right)\right)^{2}}{\left(\sum_{h} p_{x}\right)^{2}+\left(\sum_{h} p_{y}\right)^{2}+\left(\sum_{h}\left(E-p_{z}\right)\right)^{2}}
$$

where $\sum_{h}$ runs over all energy deposits (with momentum vectors $\left(p_{x}, p_{y}, p_{z}\right)$ ) not assigned to the scattered electron. In the QPM $\gamma$ is the direction of the struck quark. The variables $x$ and $Q^{2}$ are then determined by:

$$
\begin{aligned}
Q_{D A}^{2} & =4 E_{e}^{2} \frac{\sin \gamma\left(1+\cos \theta_{e}\right)}{\sin \gamma+\sin \theta_{e}-\sin \left(\gamma+\theta_{e}\right)} \\
x_{D A} & =x_{0} \frac{\sin \gamma+\sin \theta_{e}+\sin \left(\gamma+\theta_{e}\right)}{\sin \gamma+\sin \theta_{e}-\sin \left(\gamma+\theta_{e}\right)}
\end{aligned}
$$

The method is insensitive to hadronization and, to first order, is independent of the detector energy scales 58. At small values of $\theta_{e}$ or $\gamma$ the resolution in $x_{D A}$ and $Q_{D A}^{2}$ worsens. In order that the hadronic system be well measured, it is necessary to require a minimum of hadronic activity away from the beampipe. A suitable quantity for this purpose is the hadronic estimator 59 of $y$ :

$$
y_{J B}=\frac{\sum_{h}\left(E-p_{z}\right)}{2 E_{e}}
$$




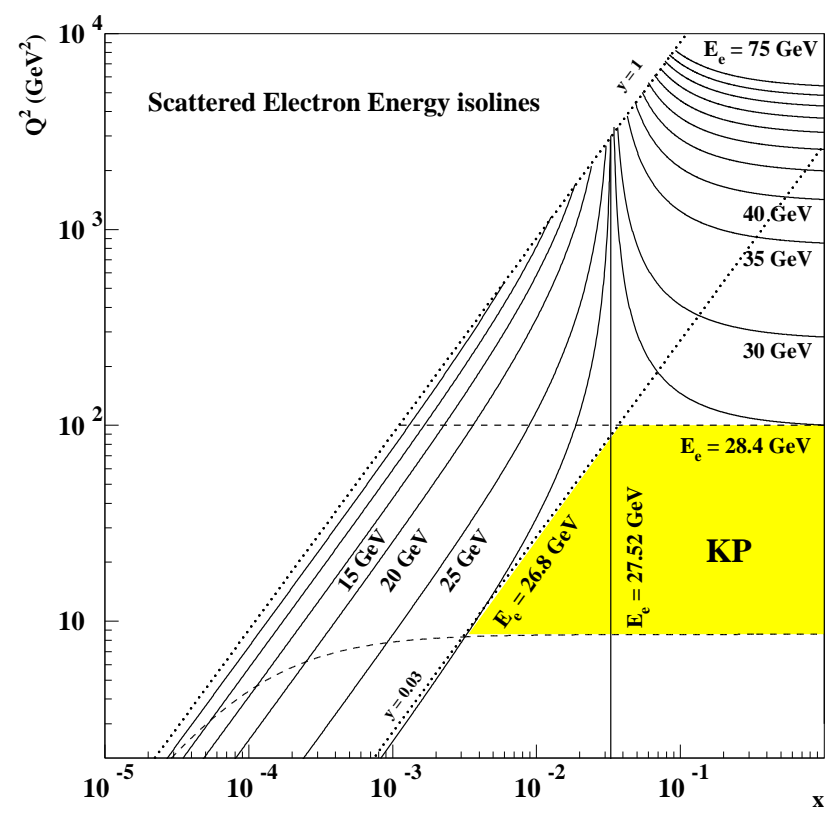

Fig. 18. Contours of constant energy of the scattered lepton in the $\left(x, Q^{2}\right)$ plane for the HERA ep collider, together with the kinematic peak (KP) region.

At low $y(<0.04)$ and low $Q^{2}$, when there is low hadronic activity in the detector the DA method becomes sensitive to noise in the calorimeter. The double angle method is also used to cross calibrate the energy scale of the detectors.

Two other methods, which use a mixture of electron and hadronic information, will be considered with the relevant experiment below.

Because of the interest in measuring the behaviour of $F_{2}$ or the total $\gamma^{*} p$ crosssection as $Q^{2} \rightarrow 0$, both ZEUS and H1 have improved and extended their detectors to be able to measure $\theta_{e}$ very close to $180^{\circ}$. In addition they have taken data from special runs of the HERA collider in which the primary interaction vertex is shifted by $70 \mathrm{~cm}$ in the proton beam direction. The interaction is thus further from the rear calorimeters which are used to identify the scattered electron and hence smaller angles can be reconstructed. Such data are referred to as shifted vertex data. Another way to reach very low $Q^{2}$ is to select a sample of events in which the incoming electron (or positron) radiates a hard collinear photon and hence provides a lower interaction energy, this technique has been used by both experiments and will be referred to as ISR.

At HERA luminosity is measured using the very small $Q^{2}$ Bethe-Heitler process $e p \rightarrow e p \gamma$, for which the cross-section is large and known very accurately. ZEUS uses lead-scintillator calorimeters and $\mathrm{H} 1$ uses KRS-15 (TlCl/TlBr) crystal cerenkov counters situated very close to the electron beam pipe in order to detect scattered beam electrons and bremsstrahlung photons. Photons emerging from the electron- 
proton interaction point (IP) at angles $\theta_{\gamma} \leq 0.5 \mathrm{mrad}$ with respect to the electron beam axis hit the photon calorimeter at $107 \mathrm{~m}(103 \mathrm{~m})$ from the IP for ZEUS (H1). Electrons emitted from the IP at scattering angles less than or equal to $6 \mathrm{mrad}$ and with energies $0.2 E_{e}<E_{e}^{\prime}<0.9 E_{e}$ are deflected by beam magnets and hit the electron calorimeter placed $35 \mathrm{~m}(33 \mathrm{~m})$ from the IP. The systematic error on the luminosity measurement is typically less than $2 \%$.

The regions in the $\left(x, Q^{2}\right)$ plane covered by the various H1 and ZEUS data sets are shown in Fig. 14. At high $Q^{2}$ the measurements are limited by event statistics. The integrated HERA luminosity collected to date is insufficient to allow measurement of structure functions for $Q^{2}>10000 \mathrm{GeV}^{2}$. However first measurements of the NC cross-section at very high $Q^{2}$ have been published and have generated great interest. They will be discussed further in Sec. 9 .

\subsection{1. $H 1$}

The H1 detector 60 for data taken until 1994 is shown in Fig. 19. The structure function measurements have relied on the inner tracking system and on the backward and liquid argon calorimeters. The inner trackers and the argon calorimeter are surrounded by a large superconducting solenoid which provides a uniform magnetic field of $1.15 \mathrm{~T}$. The 1995 detector upgrade will also be briefly mentioned.

Inner Tracker. The inner tracker consists of the forward tracker (FT) modules, a backward proportional chamber (BPC) and the central jet chamber (CJC). The CJC consists of two concentric drift chambers covering a polar angle range of $15^{\circ}-165^{\circ}$. For tracks crossing the CJC the resolution in transverse momentum is $\delta p_{T} / p_{T}<$ $0.01 p_{T}\left(p_{T}\right.$ in $\left.\mathrm{GeV}\right)$. The FT is used to determine the vertex for events which leave no track in the CJC and it covers the polar angle range of $7^{\circ}-20^{\circ}$. The BPC provides a space point, with a resolution of about $1.5 \mathrm{~mm}$ in the plane perpendicular to the beam, for charged particles entering the backward EM calorimeter and has a polar angle acceptance of $151^{\circ}-174.5^{\circ}$.

BEMC. The backward electromagnetic calorimeter covers the polar angle region $155^{\circ}-176^{\circ}$ and is of lead-scintillator construction with a depth of 22 radiation lengths, giving an energy resolution of $10 \% / \sqrt{E(\mathrm{GeV})}$.

LAR. The liquid argon calorimeter is used to measure the hadronic final state and scattered electrons at $Q^{2}>120 \mathrm{GeV}^{2}$. The LAR covers the angular range $3^{\circ}-155^{\circ}$ and has an EM section with lead absorber plates of $20-30$ radiation lengths and a hadronic section with steel absorber plates giving a total depth of 4.5-8 interaction lengths. For EM particles the energy resolution is $12 \% / \sqrt{E(\mathrm{GeV})}$, and for hadrons $50 \% / \sqrt{E(\mathrm{GeV})}$.

1995 upgrade. The H1 detector was upgraded in 199561 with the replacement of the BPC and BEMC by drift chambers and an improved calorimeter. For data with an interaction vertex shifted by $70 \mathrm{~cm}$ from the nominal position the backward drift chambers (BDC) cover the angular range $152^{\circ}-178.3^{\circ}$ and provide track segments for charged particles entering the rear calorimeter. The BEMC was replaced by a 


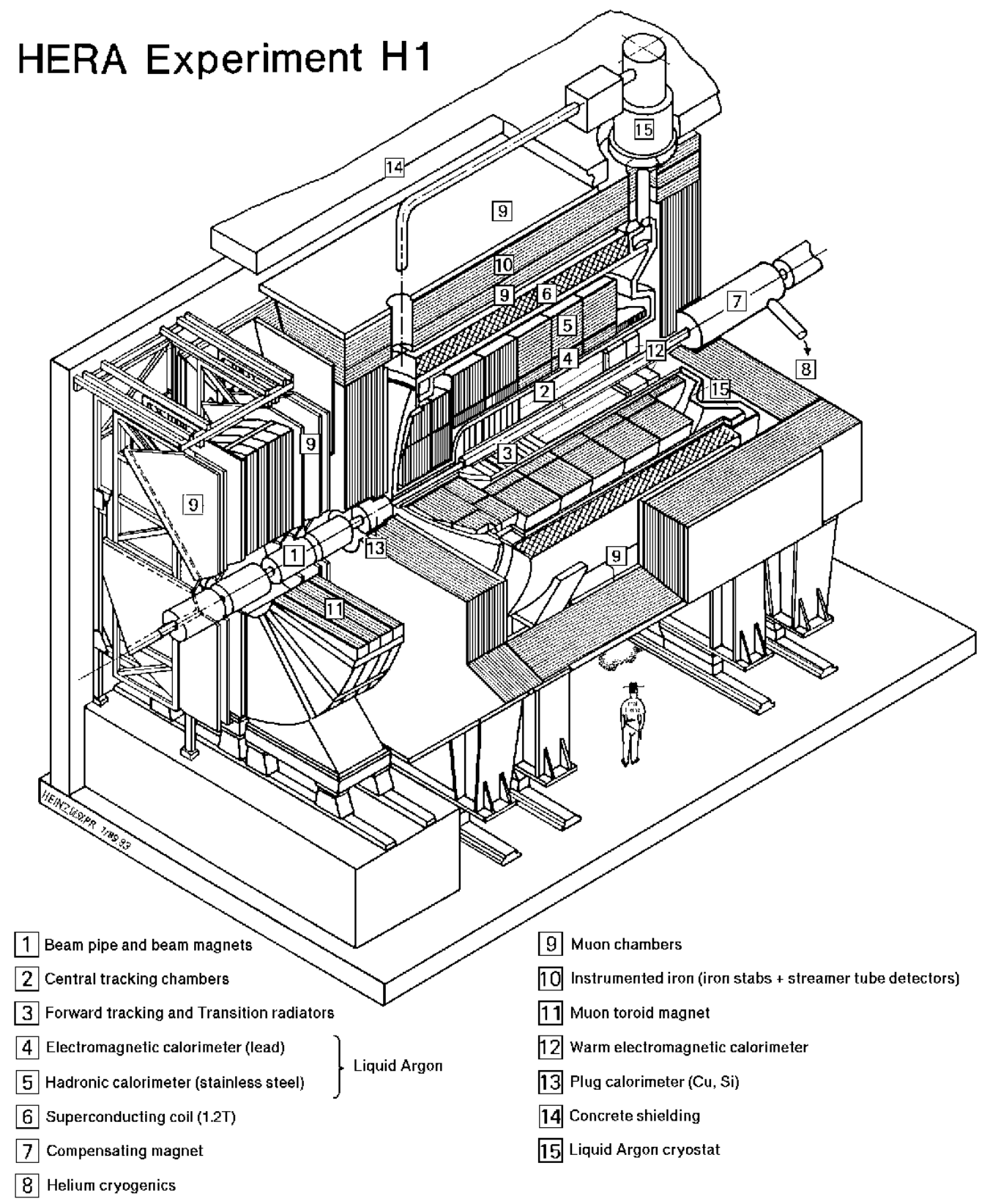

Fig. 19. The H1 detector in its configuration until end 1994. 
lead scintillating-fibre calorimeter (SPACAL) which covers the range $153^{\circ}-178.5^{\circ}$ with high granularity giving an angular resolution of $1-2$ mrad. A preliminary value for the relative energy resolution, measured during experimental operations, is $7.5 \% / \sqrt{E(\mathrm{GeV}) \oplus 2.5 \%}$. For EM energy the absolute energy scale is presently known to $1-3 \%$.

Both the BEMC and LAR electromagnetic energy scales were determined by using the observed shape of the kinematic peak and the double angle method to refine the results. The BEMC energy scale was checked to $1 \%$ and the LAR energy scale was checked to $3 \%$. The calibration of the LAR hadronic energy scale relies on test beam measurements and a comparison of the balance between the transverse energy of electrons and hadrons in DIS events. This gives a $4 \%$ uncertainty in $p_{T}^{h} / p_{T}^{e}$.

For DIS structure function measurements two triggers were used: low $Q^{2}$ required a BEMC energy cluster with $E^{\prime}>4 \mathrm{GeV}$ which was in time with the beam crossing; high $Q^{2}$ required an electromagnetic cluster in LAR with either $E^{\prime}>8 \mathrm{GeV}$ or $E^{\prime}>6 \mathrm{GeV}$ plus a track trigger. The samples collected in the 1994 HERA run were $2.2 \mathrm{pb}^{-1}$ at the nominal vertex position and $58 \mathrm{nb}^{-1}$ with the shifted vertex. Simplifying somewhat 62, after offline reconstruction DIS NC events were further required to satisfy $E^{\prime}>11 \mathrm{GeV}$, to be fully contained by either the $\operatorname{BEMC}\left(Q^{2}<120 \mathrm{GeV}^{2}\right)$ or LAR $\left(Q^{2}>120 \mathrm{GeV}^{2}\right)$ and to have a primary vertex within \pm 30 of the nominal interaction point.

For the 1995 shifted vertex sample63 of $114 \mathrm{nb}^{-1}$ the new SPACAL allowed a lower cut on $E^{\prime}$ of $7 \mathrm{GeV}$.

To improve the resolution of $x$ at small $y$ and to reduce radiative corrections H1 use the $\Sigma$ method of reconstruction 64. Returning to Eq. 112, $\sum_{h}\left(E-p_{z}\right)$ is referred to as $\Sigma$, and the essence of the idea is to remove explicit dependence on $E_{e}$ from the kinematic reconstruction of Eqs. 106 108. This is done by noting that energy and momentum conservation give $2 E_{e}=\Sigma+E^{\prime}\left(1-\cos \theta_{e}\right)$ so one defines

$$
y_{\Sigma}=\frac{\Sigma}{\Sigma+E^{\prime}\left(1-\cos \theta_{e}\right)} \quad Q_{\Sigma}^{2}=\frac{E^{\prime 2} \sin ^{2} \theta_{e}}{1-y_{\Sigma}} .
$$

The variable $x$ is then calculated from $Q^{2}=s x y$. Note that the equation for $y_{\Sigma}$ may also be written $y_{\Sigma}=y_{J B} /\left(1+y_{J B}-y_{e}\right)$. Apart from allowing an extension of the measurements to lower $y$ values than the E method, the $\Sigma$ method has a reduced sensitivity to radiative corrections.

\subsubsection{ZEUS}

ZEUS is a multipurpose detector 65 shown in Fig. 20. We give a brief description of those parts of the detector relevant for structure function measurements.

Tracking. Charged particles are tracked by the inner tracking detectors which operate in a magnetic field of $1.43 \mathrm{~T}$ provided by a thin superconducting coil. Immediately surrounding the beampipe is the vertex detector (VXD), which is a cylindrical drift chamber. Surrounding the VXD is the central tracking detector 


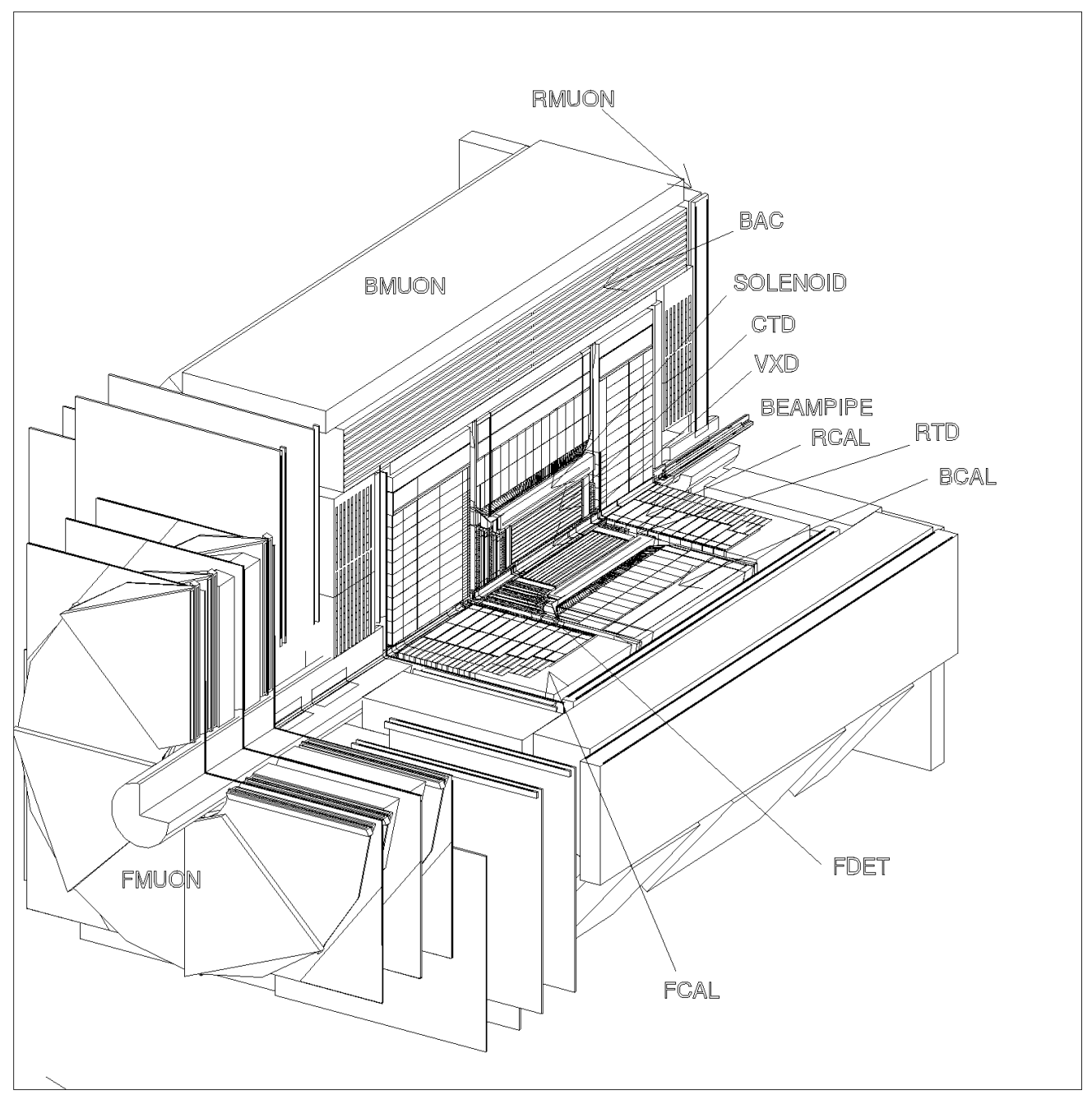

Fig. 20. The ZEUS detector. 
(CTD) which consists of 72 cylindrical drift chamber layers, giving a resolution in transverse momentum for full length tracks of $\sigma_{p_{T}} / p_{T}=0.005 p_{T} \oplus 0.016$ (for $p_{T}$ in $\mathrm{GeV}$ ). The interaction vertex is measured with a typical resolution of $0.4 \mathrm{~cm}$ along the beam direction and for tracks with momentum above $5 \mathrm{GeV}$ the extrapolated position on the inner face of the calorimeter is $0.3 \mathrm{~cm}$.

UCAL. The solenoid is surrounded by a high resolution uranium-scintillator calorimeter covering the polar angle region $2.6^{\circ}-176.2^{\circ}$. The resulting solid angle coverage is $99.7 \%$ of $4 \pi$. Under test beam conditions the UCAL has an energy resolution of $\sigma_{E}=0.35 \sqrt{E(\mathrm{GeV})}$ for hadrons and $\sigma_{E}=0.18 \sqrt{E(\mathrm{GeV})}$ for electrons. The UCAL also provides a time resolution of better than $1 \mathrm{~ns}$ for energy deposits greater than $4.5 \mathrm{GeV}$, which is used for background rejection.

SRTD. The position of positrons scattered at small angles to the positron beam direction is measured using the small angle rear tracking detector (SRTD). The SRTD consists of two planes of scintillator strips, $1 \mathrm{~cm}$ wide and $0.5 \mathrm{~cm}$ thick, arranged in orthogonal directions and read out via optical fibres and photo-multiplier tubes. The SRTD signals resolve single minimum ionizing particles and provide a position resolution of $0.3 \mathrm{~cm}$. The time resolution is less than $2 \mathrm{~ns}$ for a minimum ionizing particle.

BPC. The beam-pipe calorimeter is a small tungsten-scintillator sampling calorimeter located about $5 \mathrm{~cm}$ from the beam line just upstream of the RCAL $(294 \mathrm{~cm}$ from the interaction point). It is designed to measure scattered electrons at $\theta_{e}$ very close to $180^{\circ}$ with high precision. Position measurements are made with horizontal and vertical scintillator strips $8 \mathrm{~mm}$ wide. The energy resolution is $17 \% / \sqrt{E(\mathrm{GeV})}$ as measured in a test beam and confirmed by use of kinematic peak events. The absolute energy scale and strip-to-strip calibrations were also determined using kinematic peak events and confirmed using quasi-elastic ep $\rightarrow e p \rho^{0}$ events. The BPC was installed for the 1995 HERA physics run.

The absolute calibration error of the UCAL is estimated to be about $2 \%$ based on test-beam measurements and it is maintained by monitoring the level of natural uranium radioactivity. The main problem in the accurate determination of $E^{\prime}$ is to account properly for energy loss in dead material in front of the UCAL, typically 1.5 radiation lengths. Events selected in the kinematic peak have been used to calibrate the correction procedure for $E^{\prime} \approx 27.5 \mathrm{GeV}$ and $\theta_{e}>135^{\circ}$. QED Compton events $(e p \rightarrow e \gamma p)$ and DIS $\rho^{0}$ production also provide checks on the calibration at lower $E^{\prime}$ and smaller $\theta_{e}$. Together these methods give a resolution on $E^{\prime}$ of $\sigma / E=(20-27) \% / \sqrt{E(\mathrm{GeV})}$ depending on $\theta_{e}$.

The ZEUS pipelined trigger 66 was designed to operate with minimum deadtime. The primary trigger signals were: electromagnetic (EMC) energy above $2.5-4.8$ $\mathrm{GeV}$, depending on the position, and proper timing for an $e p$ collision measured with upstream veto counters and the SRTD. To reject photoproduction events, and proton beam-gas events originating within the detector, events were required to satisfy $\delta \equiv \sum_{i} E_{i}\left(1-\cos \theta_{i}\right)>\left(25-2 E_{\gamma}\right) \mathrm{GeV}$, where $E_{i}$ and $\theta_{i}$ are the energies and polar angles of UCAL cells and $E_{\gamma}$ is the energy deposited in the luminosity 
photon calorimeter. Simplifying 67 somewhat, selected DIS NC events are required to have a positron candidate with a fully contained shower and a track match for $\theta_{e}<135^{\circ}$. The electron must also satisfy $E^{\prime}>10 \mathrm{GeV}$ and $y_{e}<0.95$. In addition the events must satisfy $38<\delta<65 \mathrm{GeV}$. The 1994 sample corresponds to $2.5 \mathrm{pb}^{-1}$.

For analysis of their 1994 data ZEUS have used a variation on the $\Sigma$ method, the PT method, to extend coverage to smaller $y$ and hence to make an overlap with the fixed target data possible. The basic idea is first to use the balance in transverse momentum between the scattered positron and the hadronic system to correct $y_{J B}$ for hadronic energy loss, either in the beam pipe or in dead material. A correction function calculated from a Monte Carlo simulation (both of lepton-nucleon physics including hadronization and of detector response 67 ) is used. The $\Sigma$ method is then used to improve $y$ over the whole kinematic region. Finally $x$ and $Q^{2}$ are calculated using the double angle formula but with $\gamma$ calculated from

$$
\cos \gamma_{P T}=\frac{p_{T}^{e 2}-4 E_{e}^{2} y^{2}}{p_{T}^{e 2}+4 E_{e}^{2} y^{2}} .
$$

For the PT method to work it is necessary to limit the loss of hadronic transverse energy in the forward beam pipe and the cut $\frac{p_{T}^{h}}{p_{T}^{e}}>0.3-0.001 \gamma$, where $\gamma$ is calculated from Eq. 109, is applied to the data.

\subsection{Structure function extraction}

There are three general problems to be solved in the extraction of structure functions from the observed data. We consider the following in turn for the case of NC scattering: theoretical corrections for terms that cannot be measured directly; radiative corrections to the Born-level cross-section; detector resolution and acceptance corrections.

\subsubsection{Corrections for $F_{L}$ and $Z^{0}$ exchange}

The full equation relating the structure functions $F_{2}, F_{L}$ and $x F_{3}$ to the Born cross-section for $\mathrm{NC} \ell N$ scattering is given in Eq. 10. Without measurements from both $\mathrm{e}^{-}$and $\mathrm{e}^{+}$beams at high $Q^{2}$ the contribution of $x F_{3}$ cannot be isolated. In addition $Z^{0}$ exchange terms contribute to $F_{2}$. Corrections for both contributions must be made in order to extract $F_{2}^{e m}$. These corrections are only significant for the HERA experiments at $Q^{2}>1000 \mathrm{GeV}^{2}$ and they are calculated using the measured Standard Model electroweak couplings of the $Z^{0}$ and parton distributions extracted from lower $Q^{2}$ data. The corrections are not very sensitive to the choice of parton distribution. Ignoring $x F_{3}$, the cross-section may be written in terms of $F_{2}$ and $R$ as in Eq. 11 respectively. $R$ (or $F_{L}$ ) is difficult to measure since its contribution to the cross-section is suppressed by the $y^{2}$ factor. Measurements are discussed in more detail in Sec. 5.3, but note that in order to measure $F_{L}$ crosssection data at two different centre of mass energies are required. In the QPM and leading twist pQCD $R$ is predicted to be small for most of the kinematic range, 
and generally the contribution of $R$ (or $F_{L}$ ) to the cross section is small except for $y$ close to 1 . However, for experiments in which it has not been measured, it represents an important correction. Indeed, the treatment of the $F_{L}$ correction was the source of some of the apparent discrepancies between earlier fixed target $F_{2}^{\mu N}$ data sets. Corrections have usually been made by parametrizing $R$ and using Eq. 11 to extract $F_{2}$. Various strategies have been adopted: $R=0$ (naive QPM for $\left.Q^{2} \gg M_{N}^{2}\right) ; R=$ constant; $R=R_{Q C D}$ (i.e. approximating $R=F_{L} /\left(F_{2}-F_{L}\right)$ with $F_{L}$ given by Eq. (83); and $R=R_{S L A C}$ where the last is a parametrization 68 of data on $R$. This parametrization will be discussed in more detail in Sec. 5.3. As the first three methods do not the describe the data on $R$ at low $Q^{2}$ they have been discarded and all fixed target groups now use $R_{S L A C}$ (unless $R$ is measured in the same experiment). At larger $Q^{2}$ and large centre of mass energies, pQCD should be applicable and $R_{Q C D}$ is used. To calculate $R_{Q C D}$ groups have used either a recent set of parton distributions or the results of their own NLO fit to the $F_{2}$ data. Experiments now quote the prescription used for $R$ such that their values of $F_{2}$ may be recalculated appropriately if a different prescription is favoured by future data.

\subsubsection{Radiative corrections}

Radiative corrections are large for 'classic' DIS measurements based on the reconstruction of kinematics from the scattered lepton only. Expressions for the fixed target case involving only virtual photon exchange were first worked out by Mo and Tsai 69 and refined by the Dubna group were performed by a number of groups 71 and a number of computer codes developed. Recently a new code, HECTOR 22 , based on HELIOS73 and TERAD91느, has been developed for $e p$ colliders, but is also applicable to $\mu N$ scattering. It provides a framework for semi-analytical calculations using a variety of kinematic reconstruction schemes. Another important code for corrections at HERA is HERACLES HERACLES may also be interfaced through DJANGO 6 to codes simulating the DIS final state such as LEPTO77 or ARIADNE 8 .

We will not consider the full electroweak calculations here, but will concentrate on the important features of QED corrections for NC reactions. The notation for single photon emission from the charged lepton lines is shown in Fig. 21. Schematically the cross-section including radiative effects is related to the Born cross-section by an expression of the form

$$
{\frac{d \sigma^{r a d}}{d \mathbf{v}}}^{\text {rad }}=\int d \mathbf{v}^{\prime} K\left(\mathbf{v}, \mathbf{v}^{\prime}\right){\frac{d \sigma^{B o r n}}{d \mathbf{v}^{\prime}}}^{\text {Born }}
$$

where $\mathbf{v}$ and $\mathbf{v}^{\prime}$ are 2-dimensional vectors representing the kinematic variables $\left(x, Q^{2}\right)$, and $K\left(\mathbf{v}, \mathbf{v}^{\prime}\right)$ is the radiative kernel.

The corrections are of two sorts, virtual corrections and the infra-red part of real photon emission which give an overall modification to the Born cross-section and non-infra-red real photon emission in which the photon is not identified. The radiative process $e p \rightarrow e \gamma X$ is dominated by photon emission from the initial and 


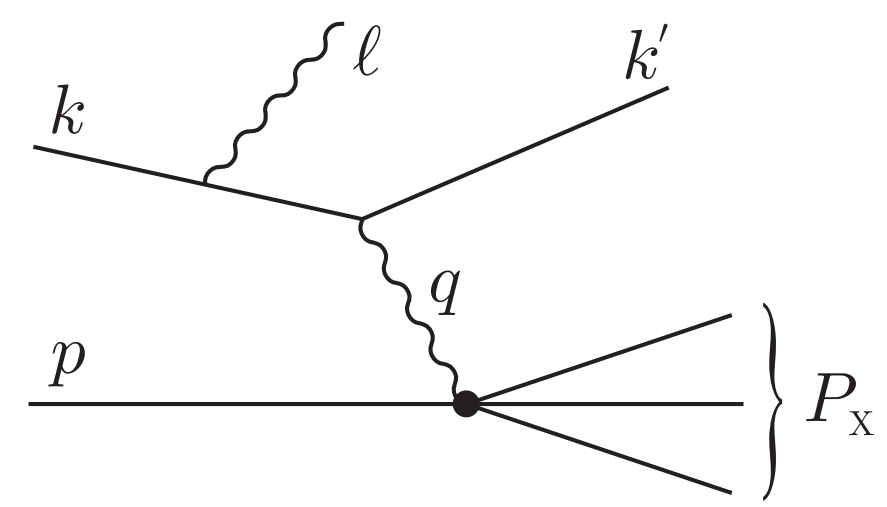

Fig. 21. Schematic diagram for radiative lepton-nucleon scattering.

final lepton. In principle there is also interference between the two. Consider first the situation when the kinematics is reconstructed using the scattered lepton only and the radiation is from the initial lepton (ISR). The standard definitions of the kinematic variables are (where $\ell$ indicates that they have been calculated from the lepton side),

$$
Q_{\ell}^{2}=-\left(k-k^{\prime}\right)^{2} \quad x_{\ell}=\frac{Q_{\ell}^{2}}{2 p \cdot\left(k-k^{\prime}\right)} \quad y_{\ell}=\frac{p \cdot\left(k-k^{\prime}\right)}{p . k} .
$$

The effect of the radiation of a photon of 4-momentum $l$, is to lower the actual centre of mass energy in the ep interaction from $s=(k+p)^{2}$ to $s^{\prime}=(k-l+p)^{2}$. The true kinematic variables are correctly reconstructed from the hadron side

$$
Q_{h}^{2}=-\left(k-k^{\prime}-l\right)^{2} \quad x_{h}=\frac{Q_{h}^{2}}{2 p \cdot\left(k-k^{\prime}-l\right)} \quad y_{h}=\frac{p \cdot\left(k-k^{\prime}-l\right)}{p \cdot(k-l)} .
$$

For collinear ISR the lepton side and true variables are related by

$$
Q_{h}^{2}=z_{i} Q_{\ell}^{2} \quad x_{h}=\frac{x_{\ell} y_{\ell} z_{i}}{y_{\ell}+z_{i}-1}
$$

where $z_{i}$ is the fractional energy loss from the initial state lepton $z_{i}=\left(E-E_{\gamma}\right) / E$. Similar expressions can be deduced in the case of collinear radiation from the final state lepton (FSR).

In terms of the variables $x$ and $y$, the radiative kernel $K$ has to be integrated over the domain $x_{\ell} \leq x \leq 1,0 \leq y \leq y_{\ell}$ (shaded region in Fig. 22). Particularly for electrons, the radiative kernel is large in three regions: (i) the 's-peak' region corresponding to collinear ISR and following a curve in the $\left(x, Q^{2}\right)$ plane given by Eq. 118 and shown as the full curve in the figure; (ii) the 'p-peak' region corresponding to collinear FSR and shown as a dash-dotted line; (iii) the 't-peak' or Compton region which occurs as $Q^{2} \rightarrow 0$ and may be viewed as Compton scattering from the almost real exchange photon. The size of the contribution from the 


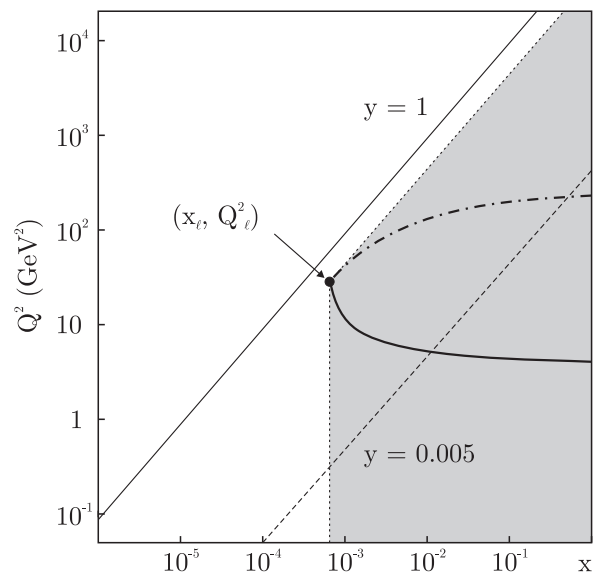

Fig. 22. Phase space for one-photon emission at fixed $\left(x_{\ell}, Q_{\ell}^{2}\right)$. The full curve shows the s-peak (photon collinear with incoming lepton) and the dash-dotted curve the p-peak (photon collinear with the outgoing lepton) as the emitted photon energy ranges from zero to its kinematically allowed maximum.

'peak' regions is characterised by $\ln \left(m_{\ell}^{2} / Q^{2}\right)$ and is thus less pronounced for muon scattering. In order to calculate the radiative corrections, one needs to know the structure functions from the point measured down to very small values of $Q^{2}$ as the shaded region in Fig. 22 shows. Not only is data poorly measured in parts of the integration domain, but it may also include some of the region that is being measured in the same experiment. The procedure adopted for fixed target experiments $\mathbb{Q}^{\mathrm{s}}$ is to use a phenomenological parametrization of $F_{2}\left(x, Q^{2}\right)$ chosen to give a good representation of the data over a wide range of $Q^{2}$ and particularly at very small values. The fit parameters may be improved during unfolding. One of the biggest uncertainties in this procedure comes from lack of knowledge of $R$. The radiative corrections are sizeable for large $y$ and small $x$, for example in the lowest $x$ bins of the E665 experiment the corrections reach $40 \%$ at the largest $Q^{2}$ values 80

At HERA, even though the lepton beam is composed of electrons or positrons, the radiative corrections turn out to be less severe. There are two reasons for this. The first is that since the scattered electron is measured by calorimetric methods the energy lost through FSR will usually be deposited in the same calorimeter cell as the electron itself. The second and more important is that kinematic information is measured from both the electron and hadron sides. Even if the hadron information is not used in the final reconstruction of $x$ and $Q^{2}$, requiring that, for example, $\left|y_{e}-y_{J B}\right|$ is less than some small value will limit the energy lost through hard radiation. In practice the variable that is often used by the HERA experiments is $\delta=\sum_{i} E_{i}\left(1-\cos \theta_{i}\right)$ where the sum runs over all calorimeter cells and $\theta_{i}$ is 
the angle with respect to the proton beam direction. For a completely hermetic detector and in the absence of ISR, $\delta=2 E_{e}$, where $E_{e}$ is the HERA $e^{ \pm}$beam energy. The measured values of $\delta$ are lowered by losses in rear beam-pipe (electron side), particularly real or nearly real photoproduction events, and ISR. Thus requiring a minimum value of $\delta$ removes both ISR events and photoproduction background. Over the region of the $\left(x, Q^{2}\right)$ plane used for $F_{2}$ measurements at HERA, radiative corrections do not exceed $10 \%$ and are typically a factor 2 smaller. A summary of radiative corrections at high $y$ for different reconstruction methods at HERA, calculated using the HECTOR code, is given in Bardin et al 11 .

\subsubsection{Extracting $F_{2}$ from the observed events}

Before $F_{2}$ values can be extracted from the measured cross-section residual backgrounds must be subtracted. Off-target scattering in the fixed target case or beamgas interaction at HERA is estimated from empty target runs (fixed target) or the use of the non-colliding bunches (HERA). In the case of HERA, background from real photoproduction processes in which a false scattered electron is identified is a serious background at high $y$. This is estimated from the shape of the $\delta$ distribution, from Monte Carlo simulations and using tagged photoproduction events.

Although there are considerable differences in detail, all experiments follow much

the same procedure in principle. $F_{2}$ is extracted from the observed distribution of events by unfolding

$$
N^{o b s}=\mathcal{L} \int_{b i n} d \mathbf{v}_{\mathbf{m}} \int d \mathbf{v} \mathcal{A}_{K}\left(\mathbf{v}_{\mathbf{m}}, \mathbf{v}\right) F_{2}(\mathbf{v})
$$

where $N^{o b s}$ is the number of observed events in a bin (after background subtraction) and $\mathcal{A}_{K}$ is a convolution of the radiative kernel $K$ and the acceptance function $A$. The quantities $\mathbf{v}$ and $\mathbf{v}_{\mathbf{m}}$ are 2 dimensional vectors representing the true and measured kinematic variables $\left(x, Q^{2}\right)$ respectively. With the advent of DIS Monte Carlo event generators incorporating radiative effects $\mathcal{A}_{K}$ is usually determined in one step. For some of the older fixed target experiments, the radiative corrections were calculated semi-analytically and only the acceptance and resolution smearing function $A$ was determined from the detector Monte Carlo code. In all cases an iterative approach is followed as corrections depend on the shape of the cross-section that is being measured.

A wide variety of unfolding methods have been proposed and more details are given in the papers by Blobel 82, D'Agostini 83 and Zech 84 . The more complicated methods are analogous to matrix inversion and using such methods one is less sensitive to the Monte Carlo simulation and the unfolded data tends to be 'smoother'. Large Monte Carlo samples are needed to guarantee the necessary stability of the result. However, in practice, if the Monte Carlo data from which the unfolding matrix is generated describes the data reasonably well the different methods give very similar results for the unfolded data 85 .

We give a very brief account of the simplest method - known as bin-by-bin un- 
folding. Starting from a recent set of parton density functions, giving $F_{2}^{\text {input }}$, DIS events are generated with the help of a lepton-nucleon physics simulation code such as LEPTO 7 , ARIADNE 8 or GMUON 8 (and as we have mentioned radiative effects may also be included in the generation). These Monte Carlos not only describe the lepton-nucleon interaction at the parton level using QCD matrix elements, they also deal with the hadronization of the final state partons. The hadronization prescriptions differ between the Monte-Carlos - the interested reader is referred to Sec. 7.7 where this is discussed in context of measurements of the structure of the hadron final state. The events are then passed through a Monte Carlo simulation of the detector (usually the most time-consuming task) and subjected to the same set of cuts as the real DIS data. Normalising the Monte Carlo data to the measured luminosity gives the predicted array $N^{M C}\left(x_{m}, Q_{m}^{2}\right)$. This is now used to correct the input structure functions using

$$
F_{2}^{\text {new }}\left(x, Q^{2}\right)=\frac{N^{\text {obs }}\left(x_{m}, Q_{m}^{2}\right)}{N^{M C}\left(x_{m}, Q_{m}^{2}\right)} F_{2}^{o l d}\left(x, Q^{2}\right),
$$

where $F_{2}^{\text {old }}=F_{2}^{\text {input }}$ initially. The $F_{2}^{\text {new }}$ values are then 'smoothed' in some way, either fitting a suitable function of $\left(x, Q^{2}\right)$ or most recently by a NLO QCD fit. The smoothed structure function is then used to re-weight the Monte Carlo events such that $F_{2}^{\text {new }}=F_{2}^{\text {input }}$ for a second iteration of the procedure, which produces a new array $N^{M C}\left(x_{m}, Q_{m}^{2}\right)$. The procedure is repeated, usually only 2-3 times, until a convergence criterion is satisfied (typically that the change in $F_{2}$ is less than $0.2-0.5 \%$ ). To account properly for the migration between bins caused by finite detector resolutions, the simulated data must describe well the key measured quantities such as the angle and energy of the scattered electron.

Strictly speaking $F_{2}$ is measured as an average over the bin area, $\Delta x \Delta Q^{2}$. However it is much more convenient to present the data at a predetermined $\left(x, Q^{2}\right)$ point in the bin rather than at $\left\langle x, Q^{2}\right\rangle_{\text {bin }}$. This requires a correction, known as bincentering, which is usually performed with the function used to smooth the data during unfolding. This introduces a small uncertainty in the quoted value of $F_{2}$.

\subsubsection{Systematic errors}

One of the most important and time consuming tasks in the extraction of structure function data is the estimation of systematic errors. This requires a good knowledge of the detector and the methods used for event reconstruction. Some uncertainties, such as those in beam flux or luminosity will affect all data by a common factor, other changes, for example energy scales or cut values, may affect the distribution of the data in a correlated way. To estimate these latter effects, a change is made and the complete structure function extraction repeated so that the changes $\delta F$ can be determined. Most experimental teams now try to group the systematic errors into classes that are more or less independent and some have tried to parameterize the correlated errors. The actual values of the full correlated systematic errors are usually available from the experimental groups. The 
phenomenological fits done by experimental groups take full account of their own systematic errors but the problem of how to do this between experiments and for global parton density determinations, such as those discussed in Sec. 6.1.2, is still a subject of active discussion 87 .

\section{The data}

In this section we consider the structure function data in some detail, covering the range and quality of the data and the consistency between the different experiments. A summary is given in Table 2. All experiments measure $F_{2}$, those indicated have measured $R$ and only the CCFR experiment (of the ones we consider) has measured $x F_{3}$. We note in passing that all the fixed target experiments have measured the structure functions for nuclear targets as well as $p$ and $d$. Apart from the CCFR experiment we do not consider such data in this review.

Table 2. Summary of data from recent structure function experiments. An entry in the $R$ column indicates that $R$ was measured. All the data referred in this table are available from the Durham HEPDATA database, at http://durpdg.dur.ac.uk/HEPDATA on the world wide web.

\begin{tabular}{|c|c|c|c|c|c|c|}
\hline$\overline{\operatorname{Beam}(\mathrm{s})}$ & Targets & Experiment & $Q^{2}\left(\mathrm{GeV}^{2}\right)$ & $x$ & $R$ & $R_{\text {fff }}$ \\
\hline$e^{-}$ & $\mathrm{p}, \mathrm{d}, \mathrm{A}$ & SLAC & $0.6-30$ & $0.06-0.9$ & $\sqrt{ }$ & 990 \\
\hline$\mu$ & $\mathrm{p}, \mathrm{d}, \mathrm{A}$ & BCDMS & $7-260$ & $0.06-0.8$ & $\sqrt{ }$ & 2] 2 \\
\hline$\mu$ & $\mathrm{p}, \mathrm{d}, \mathrm{A}$ & $\mathrm{NMC}$ & $0.5-75$ & $0.0045-0.6$ & $\sqrt{ }$ & 9 \\
\hline$\mu$ & $\mathrm{p}, \mathrm{d}, \mathrm{A}$ & E665 & $0.2-75$ & $8 \cdot 10^{-4}-0.6$ & & 7 \\
\hline$\nu, \bar{\nu}$ & $\mathrm{Fe}$ & CCFR & 1. -500 . & $0.015-0.65$ & $\sqrt{ }$ & $5 . \mid 106$ \\
\hline$e^{ \pm}, \mathrm{p}$ & - & $\mathrm{H} 1$ & $0.35-5000$ & $6 \cdot 10^{-6}-0.32$ & - & 63163 \\
\hline$e^{ \pm}, \mathrm{p}$ & - & ZEUS & $0.16-5000$ & $3 \cdot 10^{-6}-0.5$ & - & 100, 67, 102 \\
\hline
\end{tabular}

When there is no ambiguity we shall refer to a dataset simply by the collaboration acronym or code name (SLAC, BCDMS, E665). For NMC and CCFR datasets we qualify the acronym by year of publication of the data, e.g. NMC(95), but note that the data may sometimes appear in global fits a year earlier. For HERA datasets we qualify the acronym by the year in which the data was collected, thus H1(94) etc.

\section{1. $F_{2}^{\mu N}$ and $F_{2}^{e N}$}

The overall situation on the data for $F_{2}$ is rather pleasing. The fixed target programme is complete and the final results published. Most of the fixed target results at low and moderate $Q^{2}$ are systematics limited and the level of understanding achieved (better than 5\% systematic errors in many cases) is a tribute to the hard work over many years of the experimental teams. The major inconsistencies between different experiments, particularly EMC versus BCDMS, have been resolved 88 (in favour of BCDMS). The other major advance in the field is the addition of data from HERA and in particular the first high statistics data from the 1994 data taking period. The reach in the $\left(x, Q^{2}\right)$ has improved by two orders of magnitude at both low $x$ and high $Q^{2}$. The data from H1 and ZEUS are also 
nicely consistent with each other and with fixed target data. The use of improved $x$ and $Q^{2}$ reconstruction in the analysis of the 1994 data has given a small region of overlap in which a direct check on the relative normalisation of the collider and fixed target results may be made. A large overlap region will only be possible if HERA is run at a lower centre of mass energy. Until recently fits to determine global parton distribution functions and QCD parameters were dominated by the high statistics fixed target data, particularly BCDMS and NMC. Now the HERA data have a statistical weight that is beginning to match that of the other experiments and as systematic errors diminish, the data are starting to have an influence outside the HERA kinematic region.

\subsubsection{Fixed target $F_{2}^{\mu N}$ data}

In addition to the experiments covered in detail in this review we note that structure function data from two of the earlier fixed target experiments are still used in the determination of parton distributions. They are the SLAC experiments, following the re-analysis by Whitlow et al $\mathrm{g} 9$ in 1992, and the high statistics BCDMS experiment.

Taken together the 8 SLAC ep and ed experiments provide data on $F_{2}^{p}$ and $F_{2}^{d}$ in the ranges $0.06 \leq x \leq 0.90$ and $0.6 \leq Q^{2} \leq 30 \mathrm{GeV}^{2}$ (SLAC dataset). The normalisation uncertainties of the SLAC data are $\pm 2.1 \%$ for proton data and $\pm 1.7 \%$ for deuteron data. Relative normalization between the different SLAC experiments is $\pm 1.1 \%$ and the other systematic uncertainties are at the level of $0.5-2.0 \%$ from radiative corrections and $R$. Full details are given by Whitlow 90 .

BCDMS -1 studied $\mu p$ and $\mu d$ scattering at CERN using muon beam energies of $100,120,200$ and $280 \mathrm{GeV}$ giving data on $F_{2}^{p}$ and $F_{2}^{d}$ in the ranges $0.06 \leq x \leq 0.80$ and $7 \leq Q^{2} \leq 260 \mathrm{GeV}^{2}$ (BCDMS dataset). The systematic uncertainty in the absolute cross-section normalisation is $\pm 3.0 \%$ and the uncertainty in the relative normalisation between data taken at different beam energies is $\pm 1 \%$. The relative uncertainty in the finite resolution of the spectrometer is $5 \%$ and other uncertainties are less than $1 \%$. More details are to be found in ref. 22 .

The NMC collaboration has published data 93 at all muon beam energies (90, 120, 200 and $280 \mathrm{GeV}$ ) using the large angle T1 trigger (NMC(95) dataset). For this data $R_{S L A C}$ was used in the extraction of $F_{2}$. More recently NMC has presented results from the small angle T2 trigger at beam energies of 200 and $280 \mathrm{GeV} 94,95$. The small angle trigger data set covers the kinematic range $0.002<x<0.14$ and $0.5<Q^{2}<25 \mathrm{GeV}^{2}$. The enlarged data set permits the determination of $R$ as well as $F_{2}$ for both the proton and deuteron. The full range in $x$ and $Q^{2}$ now spanned by NMC $F_{2}$ data is $0.002<x<0.6$ and $0.5<Q^{2}<75 \mathrm{GeV}^{2}$, and this is referred to as the NMC(97) dataset. These are the data shown in figures unless otherwise stated. The consistency of the data taken with different triggers and beam energies is shown for $F_{2}^{p}$ in Fig. 23. The figure also shows the characteristic features of pQCD scaling violations, with the slope $\frac{\partial F_{2}}{\partial \ln Q^{2}}$ increasing as $x$ decreases. To give the final $\mathrm{NMC}(97)$ data, data taken with different triggers and beam energies are 

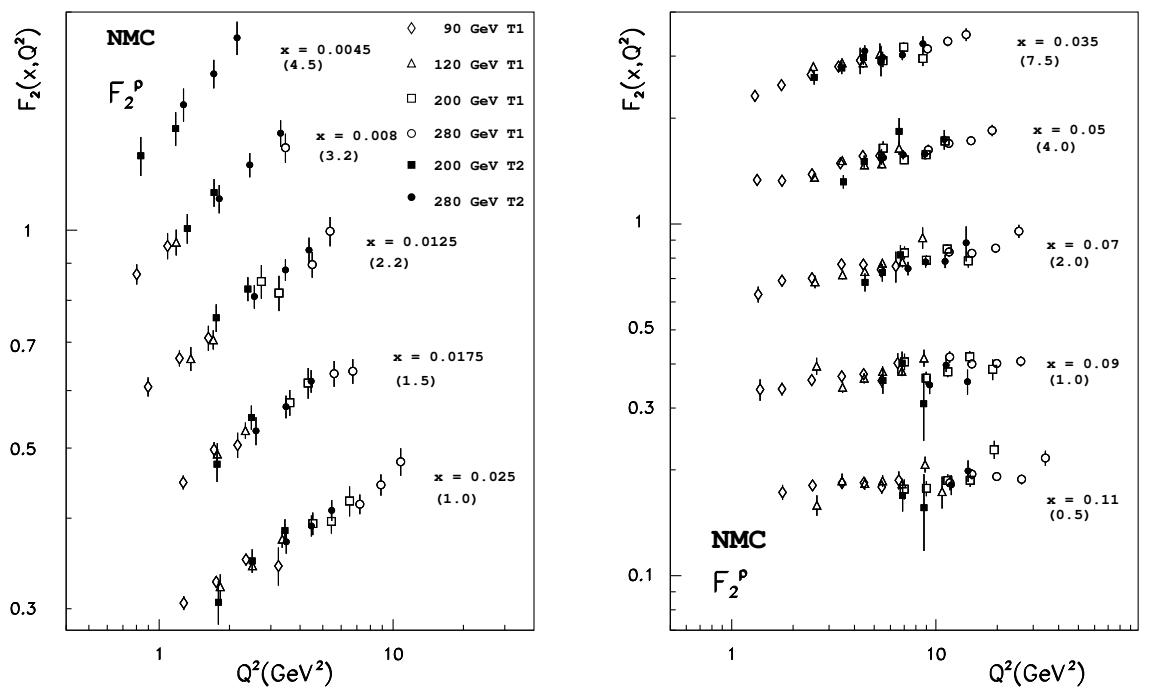

Fig. 23. $\mathrm{NMC}(97) F_{2}^{p}$ data at different muon beam energies and triggers before averaging. The inner error bar shows the statistical error and the full error bar the total error (statistical error and systematic error added in quadrature). The data in each $x$ bin are scaled by the factors indicated in brackets.

averaged where appropriate.

Two methods were used to extract $F_{2}$ values from the data. Method A used $R_{S L A C} 68$ and in method $\mathrm{B} F_{2}$ and $R$ were both extracted from the data. More details on the second method will be given in Sec. 5.3 below. The overall normalization uncertainty is $\pm 2.5 \%$. Other systematic errors are divided into five independent classes (incident beam energy, scattered muon energy, acceptance, radiative corrections and reconstruction) which are then added in quadrature to give the total systematic error on each $F_{2}$ measurement. Radiative corrections are calculated using the formalism of the Dubna group 0 . Typically the total systematic error is less than $2 \%$ but it can rise to $4 \%$ on the boundaries of the measured region in $\left(x, Q^{2}\right)$ at a given energy. To estimate precise relative normalizations between their own data and those from SLAC and BCDMS, the NMC group use a convenient parametrization of $F_{2}^{p}$ and $F_{2}^{d}$ (and their uncertainties) of the form 96

$$
F_{2}\left(x, Q^{2}\right)=A(x)\left(\frac{\ln \left(Q^{2} / \Lambda^{2}\right)}{\ln \left(Q_{0}^{2} / \Lambda^{2}\right)}\right)^{B(x)}\left(1+\frac{C(x)}{Q^{2}}\right)
$$

where $Q_{0}^{2}=20 \mathrm{GeV}^{2}, \Lambda=0.250 \mathrm{GeV}$ and $A(x), B(x), C(x)$ are simple functions of $x$ with a total of 15 parameters. To determine the parameters the data points were fit using their statistical errors and adjusting the normalization of each experiment within its quoted range. The total uncertainties in the parameterized structure functions were determined by taking into account the systematic errors of the data, 

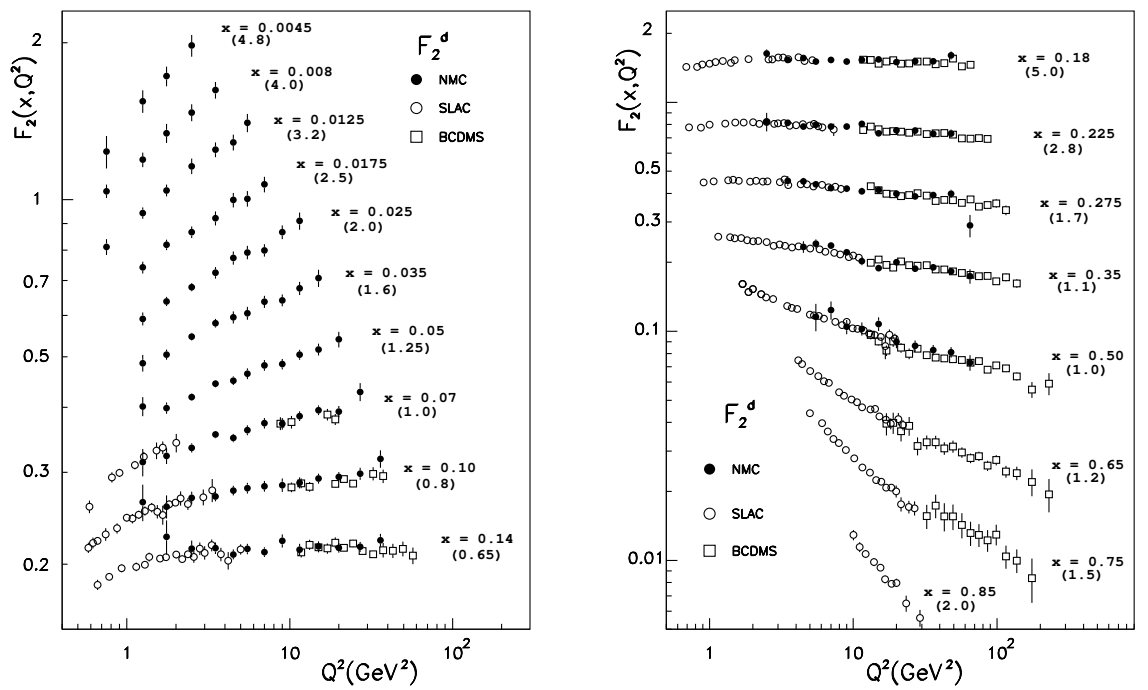

Fig. 24. A comparison of $F_{2}^{d}$ from the NMC(97), SLAC and BCDMS datasets. The SLAC and BCDMS data were rebinned to the NMC $x$ bins. The data in each $x$ bin are scaled by the factors indicated in brackets. The error bars represent the total error, apart from the overall normalization. Relative normalization is discussed in the text.

including correlations. The normalization shifts are given in Table 3. The data from the three different experiments are well consistent with each other as can be seen from Fig. 24.

Table 3. Normalization shifts for SLAC, BCDMS and NMC data from the fit using Eq. 121. The quoted overall normalization uncertainties are $2 \%, 3 \%$ and $2 \%$ for the SLAC, BCDMS and NMC experiments respectively.

\begin{tabular}{lccc}
\hline Data set & proton & deuteron \\
& & both & \\
\hline SLAC & $-0.4 \%$ & & $+0.9 \%$ \\
BCDMS & $-1.8 \%$ & & $-0.7 \%$ \\
NMC 90 GeV & & $-2.7 \%$ & \\
NMC 120 GeV & & $+1.1 \%$ & \\
NMC 200 GeV T1 & $+1.1 \%$ & \\
NMC 280 GeV T1 & & $+1.7 \%$ & \\
NMC 200 GeV T2 & & $-2.9 \%$ & \\
NMC 280 GeV T2 & $+2.0 \%$ & \\
\hline
\end{tabular}

The E665 collaboration has recently published the full analysis of data on $F_{2}^{d}$ and $F_{2}^{p}$ at a mean muon beam energy of $470 \mathrm{GeV} 90.80$. The data were collected using only the small angle trigger (see Sec. 4.1.2) and give a coverage of $0.0008<x<0.6$ and $0.2<Q^{2}<75 \mathrm{GeV}^{2}$ (E665 dataset). To extract $F_{2}$ from the measured crosssections, $R_{S L A C}$ was used, but $R=0$ and $R=R_{Q C D}$ were also used in the estimate 

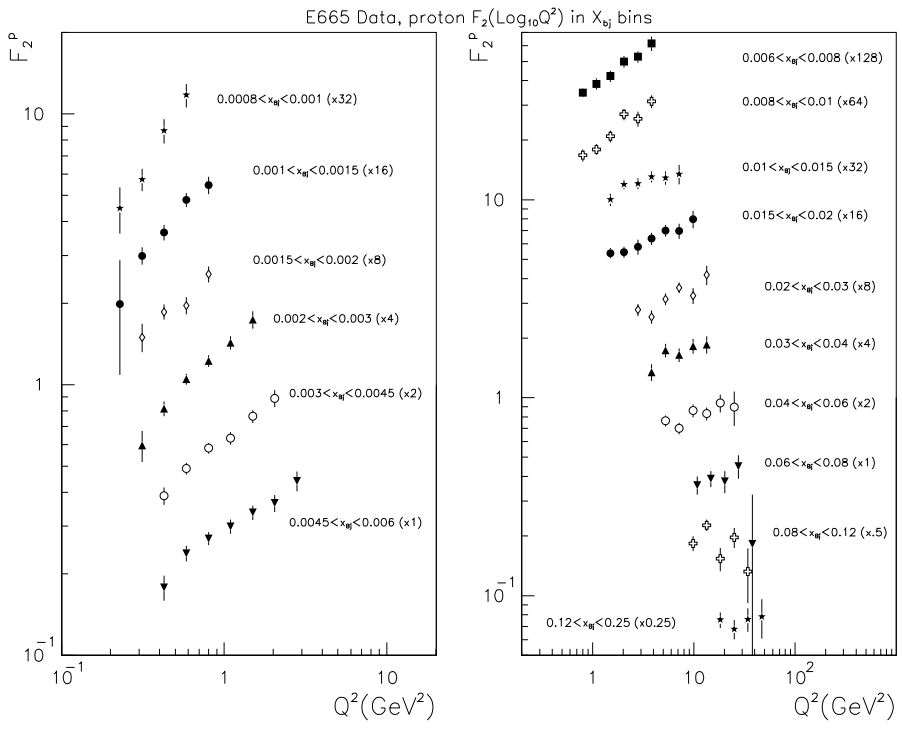

Fig. 25. $F_{2}^{p}$ data from the E665 experiment in bins of $x$ as a function of $Q^{2}$. The errors shown are the sum in quadrature of the statistical and systematic errors. The data in each $x$ bin are scaled by the factors indicated in brackets.

of systematic errors. Radiative corrections were calculated using the same formalism as used by the NMC experiment. The uncertainties in the overall normalizations are $\pm 1.8 \%$ and $\pm 1.9 \%$ for the proton and deuterium data respectively. Other systematic errors are considered in seven categories (trigger, reconstruction, absolute energy scales, relative energy scales, radiative corrections, $R$, bin centering). The total systematic error is formed by adding the errors from the seven sources in quadrature. The largest sources of uncertainty are from the trigger and reconstruction at low $x$ and $Q^{2}$. In this region the total systematic error can reach $50 \%$ at the boundaries of the measured region. Away from this region the typical total systematic error is around $3 \%$ and is often comparable to the statistical error. Fig. 25 shows the data for $F_{2}^{p}$ as a function of $Q^{2}$. Very large scaling violations at small $x$, already seen in the NMC data, are manifest.

There is a large overlap in the $\left(x, Q^{2}\right)$ regions explored by E665 and NMC and in this common region the data from the two experiments agree well both in shape and normalization - this is shown in Fig. 26. Because of the very small $Q^{2}$ values reached by E665, this data has been important in testing models for $F_{2}$ as $Q^{2} \rightarrow 0$.

\subsubsection{HERA $F_{2}^{e N}$ data}

The first $F_{2}$ measurements at HERA (HERA(92)) were made using 1992 data 98 and gave the first indication of the steep rise of $F_{2}$ as $x$ decreases. The rise was confirmed by the much improved data from the 1993 run (HERA(93)) 99 . We shall concentrate here on the first really large samples provided by the $1994 \mathrm{run}$. In 


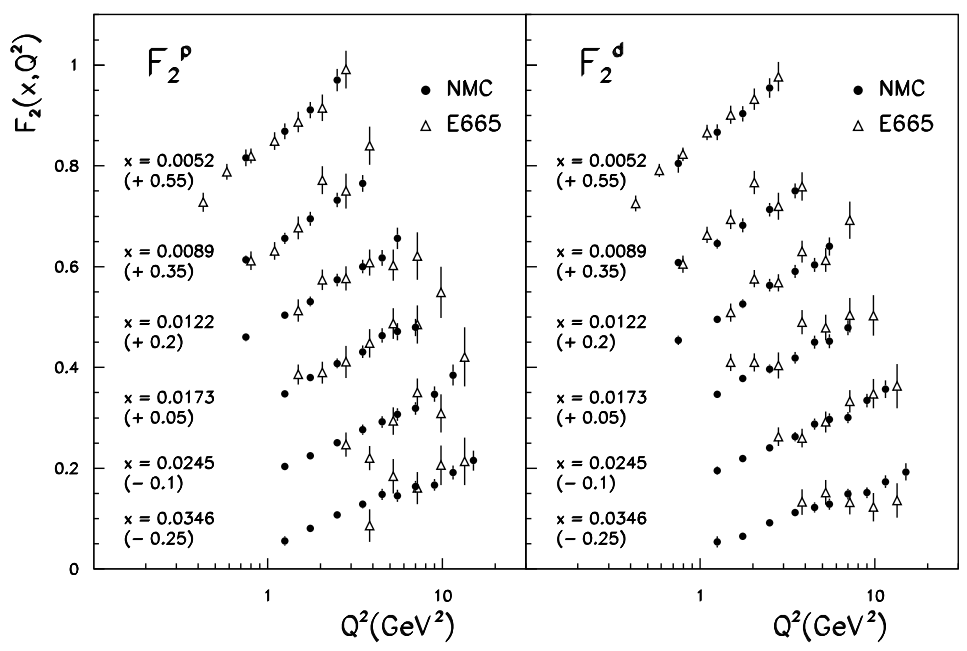

Fig. 26. A comparison of $F_{2}^{p}$ data from the E665 and NMC(97) datasets. The data in each $x$ bin are shifted by the constants indicated in brackets.

1992-93 HERA collided electrons and protons. From 1994 positrons have been used instead of electrons as larger currents could be kept with longer lifetimes. To exploit the full potential of HERA at very high $Q^{2}$ large samples of both $e^{-} p$ and $e^{+} p$ data with and without polarization will eventually be required.

The ZEUS collaboration has published their 1994 results in two papers, the first 100 contains the low $Q^{2}$ data from $58 \mathrm{nb}^{-1}$ of shifted vertex data (SVX) and initial state radiation events (ISR) and the second 67 the analysis of the large nominal vertex sample (NVX), together giving the ZEUS(94) dataset. The low $Q^{2}$ data covers the kinematic region $1.5<Q^{2}<15 \mathrm{GeV}^{2}, 3.5 \cdot 10^{-5}<x<0.004$ and events are reconstructed using the E method. $R$ is calculated using $R_{Q C D}$ (as calculated to lowest order from Eq. 83) with parton distributions determined from the QCD NLO fit used to smooth the data during unfolding. Sources of systematic error considered are: positron finding and energy scale; positron angle; photoproduction background; primary vertex reconstruction; varying the $\delta$ cut. The total systematic error varies between 4 and 14\% (compared to the statistical error which is in the range $4-9 \%$ ). The overall normalization error from the trigger and luminosity is $3 \%$. The ISR analysis used the full 1994 sample and events were selected with a modification of the DIS selection to require a photon with energy satisfying $6<E_{\gamma}<18 \mathrm{GeV}$ tagged in the luminosity photon calorimeter. The $\delta$ cut was replaced by a cut on $\delta^{\prime}=\delta+2 E_{\gamma}$. The main source of background for this technique, which is the random coincidence of a DIS or photoproduction event with a bremsstrahlung event $e p \rightarrow e p \gamma$, is estimated from a sample of events selected without the tagged photon to which a photon is added with an energy selected from a random sampling of the photon energy spectrum of bremsstrahlung events. The level of this background is 
below $10 \%$ except in the lowest $x$ bins where it rises to a maximum of $24 \%$.

The ZEUS NVX sample67 covers the region $3.5<Q^{2}<5000 \mathrm{GeV}^{2}, 6.3 \cdot 10^{-5}<$ $x<0.5$, and events were reconstructed using the PT method. First order radiative effects were included in event simulation using HERACLES. Higher order QED corrections, such as soft photon exponentiation not included in HERACLES, were calculated using the programme HECTOR. These additional corrections are small $(0.2-0.5 \%)$ and vary smoothly with $Q^{2}$. Systematic errors are grouped into six categories: positron finding; positron angle; positron energy scale; hadronic energy scale and noise; hadronic energy flow and photoproduction background. For the majority of bins the total systematic error is below $5 \%$ but increases to around $10 \%$ as $y$ increases above 0.5 . The overall normalization uncertainty is $2 \%$. $R$ is calculated from $R_{Q C D}$ as in the ZEUS SVX analysis and at high $Q^{2}$ the data are corrected to give $F_{2}$ as explained in Sec. 4.3.1. The ZEUS data are shown in Figs. 27 and 28, which show the strong rise in $F_{2}$ as $x$ decreases.

H1 have published results from their 1994 sample using NVX, SVX and ISR data 62 (H1(94) dataset). The $\mathrm{E}$ and $\Sigma$ kinematic reconstruction methods were used at low $(<0.15)$ and high $(>0.15) y$ respectively to give an overall $x$ resolution better than $20 \%$. The coverage in $\left(x, Q^{2}\right)$ from all methods is $1.5<Q^{2}<5000 \mathrm{GeV}^{2}$ and $3 \cdot 10^{-5}<x<0.32$. For the ISR data a photon with energy $E_{\gamma}>4 \mathrm{GeV}$ was required, and the minimum electron energy was lowered to $E_{e}=8 \mathrm{GeV}$. To separate signal from background events overlapping with bremsstrahlung events, the quantity $\left(E_{\gamma}-E_{e}\left(y_{e}-y_{h}\right)\right)$ was required to be less than 0.5 (with $y_{e}$ and $y_{h}$ defined in Eqs. 106 and 112). The remaining background amounts to an average of $8 \%$, with at most $15 \%$ in one $\left(x, Q^{2}\right)$ bin, and it was statistically subtracted. For the value of $R$ the QCD prediction was taken with the GRV parton distributions as input. The following sources of systematic errors were considered: positron energy scale; hadronic energy scale; positron angle; vertex reconstruction; radiative corrections; photoproduction background. The typical total systematic error is around $5 \%$. For the ISR analysis there is an additional uncertainty of around $2 \%$ from the geometrical acceptance and relative energy calibration of the luminosity photon tagger. For the NVX and SVX samples the overall normalization uncertainties (from trigger and luminosity measurement) are $1.5 \%$ and $3.9 \%$ respectively. First order radiative effects were included in the Monte Carlo simulation events using the HERACLES code. These were checked using the HECTOR programme. The data are shown in Figs. 27 and 28. The data points include also the high $y$ measurements made by H1 101, primarily used to extract $R$, as discussed in Sec. 5.3.

The $F_{2}^{p}$ data from the two HERA experiments are nicely consistent with each other and appear to connect smoothly to data from the fixed target experiments. This is shown in Fig. 29, which also shows a NLO DGLAP QCD fit. Using the extended kinematic reconstruction methods for HERA data now gives a small region of overlap with the fixed target regime in both $x$ and $Q^{2}$. Fig. 30 shows the comparison in more detail for the region $0.0025<x<0.14$. The absolute normalizations of the data from the two types of experiment are in good agreement. 


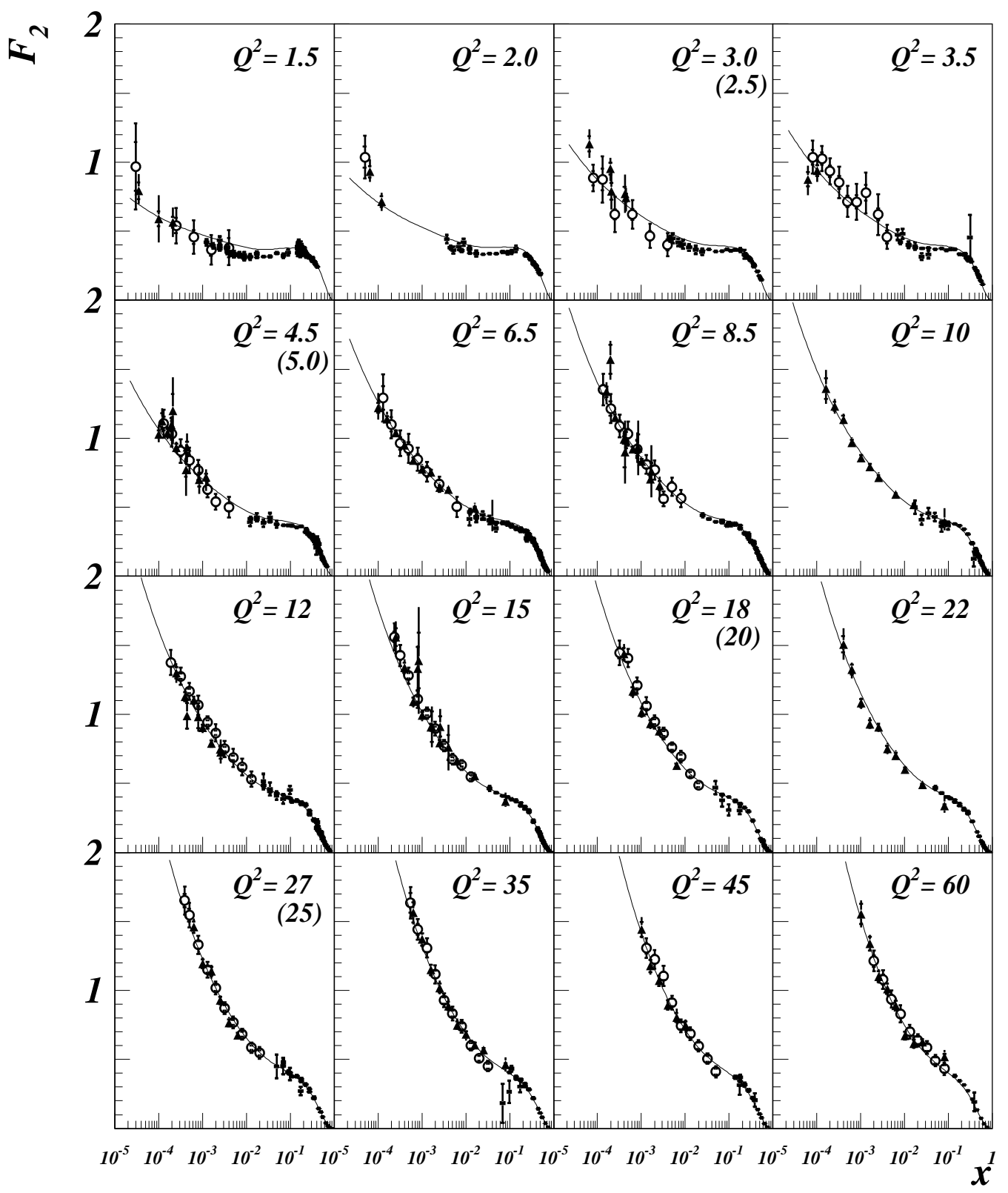

Fig. 27. ZEUS(94) and H1(94) $F_{2}^{p}$ data at fixed $Q^{2} \leq 60 \mathrm{GeV}^{2}$. The curves shown are the NLO DGLAP QCD fit used to smooth the data during unfolding. 


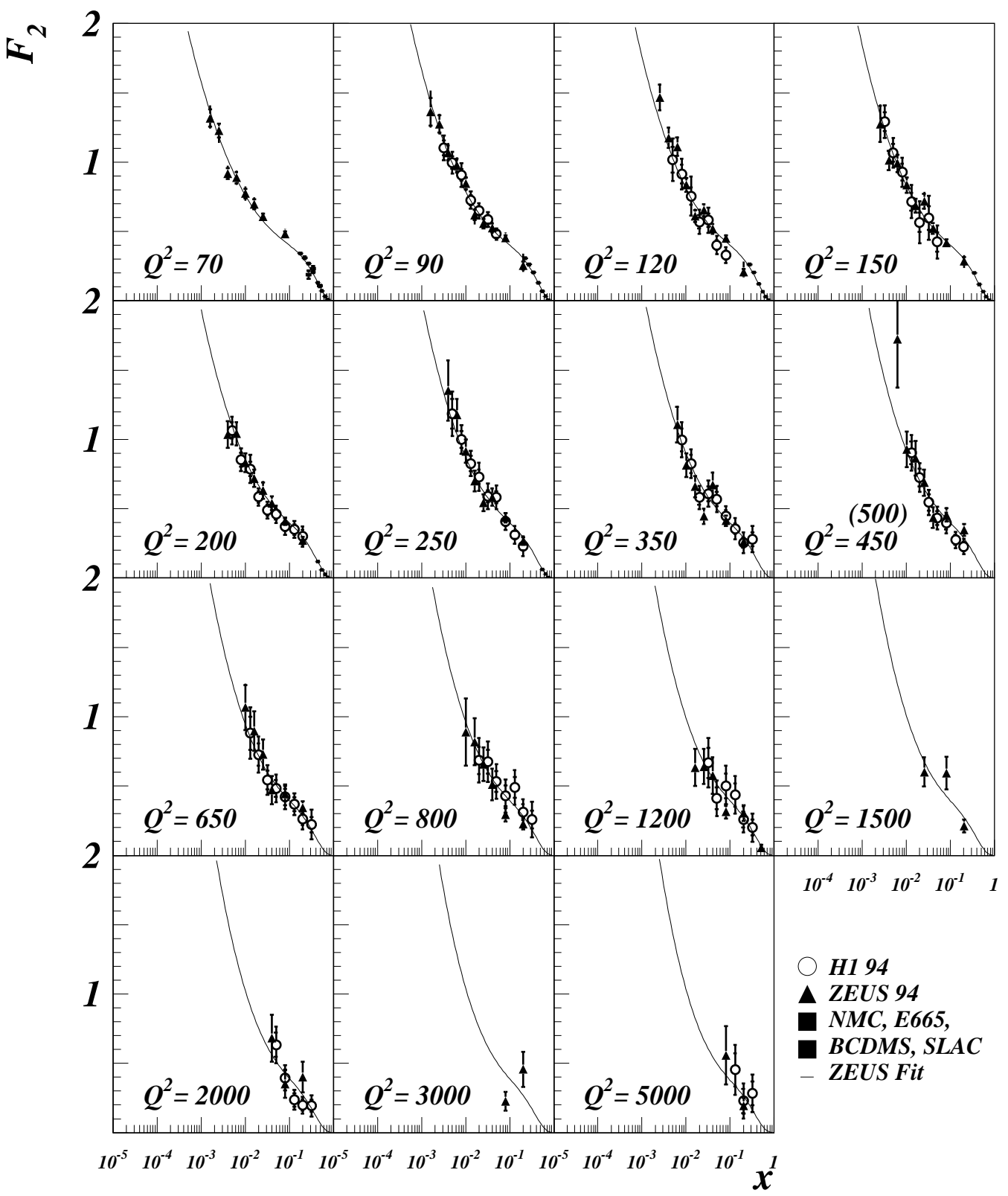

Fig. 28. ZEUS(94) and H1(94) $F_{2}^{p}$ data at fixed $Q^{2} \geq 60 \mathrm{GeV}^{2}$. The curves shown are the NLO DGLAP QCD fit used to smooth the data during unfolding. 
This is a significant achievement and is of considerable importance when it comes to combining fixed target and HERA data for further physics analysis.

The major new information on structure functions from HERA is the very strong rise of $F_{2}$ as $x$ decreases. This has now been measured with considerable precision. The slope $\frac{\partial F_{2}}{\partial x}$ does decrease as $Q^{2}$ decreases, but there is still a noticeable rise at $Q^{2}$ values as low as $1.5 \mathrm{GeV}^{2}$. The apparent success of NLO QCD fits in describing the data over the $Q^{2}$ range $1.5-5000 \mathrm{GeV}^{2}$ and for $x$ values down to $3.5 \times 10^{-5}$ as shown in the figures above, is also somewhat surprising and will be discussed in greater detail in Sec. 7 below.

As $Q^{2} \rightarrow 0 F_{2} \rightarrow 0$ and at some smallish value of $Q^{2}$ the pQCD description afforded by the DGLAP evolution must breakdown. The surprise at HERA is that the size of the transition region, from photoproduction to the deep inelastic regime, is so narrow. To measure structure functions at very small $Q^{2}$ requires a detector that can be triggered by, and can reconstruct, the scattered muon or electron at very small scattering angles. Of the fixed target experiments E665 has this capability and has published $F_{2}$ data down to $Q^{2}=0.2 \mathrm{GeV}^{2}$. At HERA the first attempt to explore this region was by use of SVX data, but that still had a lower limit of about $1.5 \mathrm{GeV}^{2}$. In 1995, to cover the transition region $0<Q^{2}<1.5 \mathrm{GeV}^{2}$, ZEUS 102 added a special detector near the rear beam-pipe and the new rear detectors of $\mathrm{H} 163$ gave them improved coverage at small electron scattering angles.

The ZEUS BPC data (ZEUS(95)BPC data set) covers the region $0.11<Q^{2}<$ $0.65 \mathrm{GeV}^{2}, 2 \cdot 10^{-6}<x<6 \cdot 10^{-5}$ and is based on $1.65 \mathrm{pb}^{-1}$ data collected in 1995 . The largest systematic errors are the position and energy scale of the BPC and the estimate of the photoproduction background. The total systematic error varies from $6 \%$ to $11 \%$ at the highest $y$ values. In addition there is an overall normalization uncertainty of $2.4 \%$.

ZEUS announced preliminary low $Q^{2}$ data from the 1995 shifted vertex run (ZEUS(95)SVX) 103 at the DIS97 meeting. The method used was essentially the same as that described above for the 1994 SVX analysis, the primary interaction point was shifted $70 \mathrm{~cm}$ in the proton beam direction. The increased statistics from $236 \mathrm{nb}^{-1}$ allowed $F_{2}$ to be extracted in seven $Q^{2}$ bins between 0.65 and $4.64 \mathrm{GeV}^{2}$.

Using their new rear detector and the $1995 \mathrm{SVX}$ sample of $114 \mathrm{nb}^{-1}$, H1 cover the region $0.35<Q^{2}<3.5 \mathrm{GeV}^{2}$ and $x>6 \cdot 10^{-6}$ (H1(95)SVX data set). The estimate of systematic errors are in the range $5-10 \%$, from positron energy and angle determination, radiative corrections and photoproduction background. There is an overall $3 \%$ normalization error from the luminosity measurement. The very low $Q^{2} F_{2}$ data are shown in Fig. 31. The phenomenology of this data will be discussed in Sec. 8 .

\section{2. $F_{2}^{\nu N}$ and $x F_{3}^{\nu N}$}

Accurate $\nu \mathrm{Fe}$ structure function data have been available for some time from the high statistics CCFR experiment 104105 (CCFR(93) dataset). Details of the detector and the calibration procedures have been given in Sec. 4.1.3. Recently 


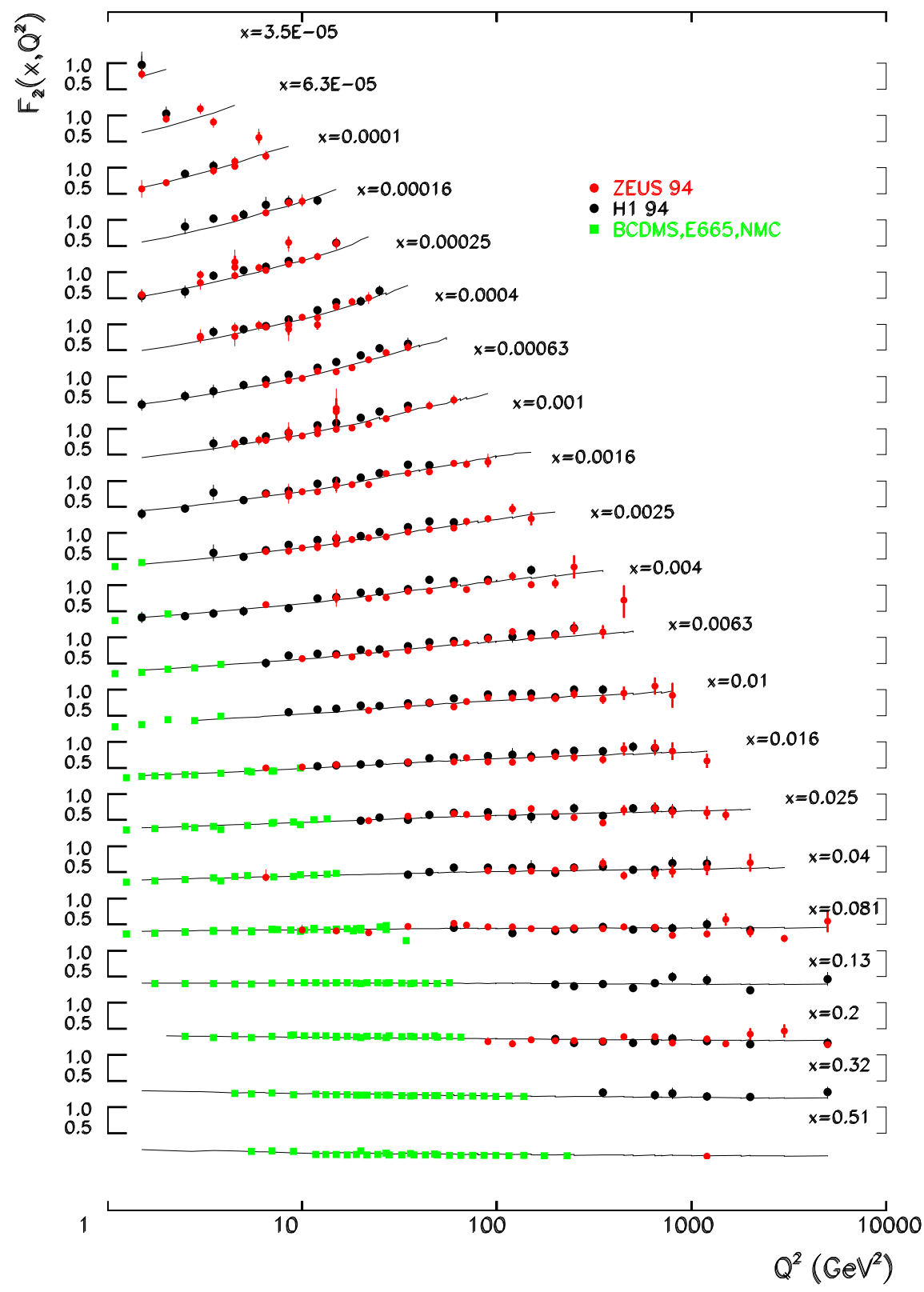

Fig. 29. $F_{2}^{p}$ data from HERA(94) and fixed target experiments at fixed $x$ as a function of $Q^{2}$. The curves shown are the NLO DGLAP QCD fit used to smooth the data during unfolding. 


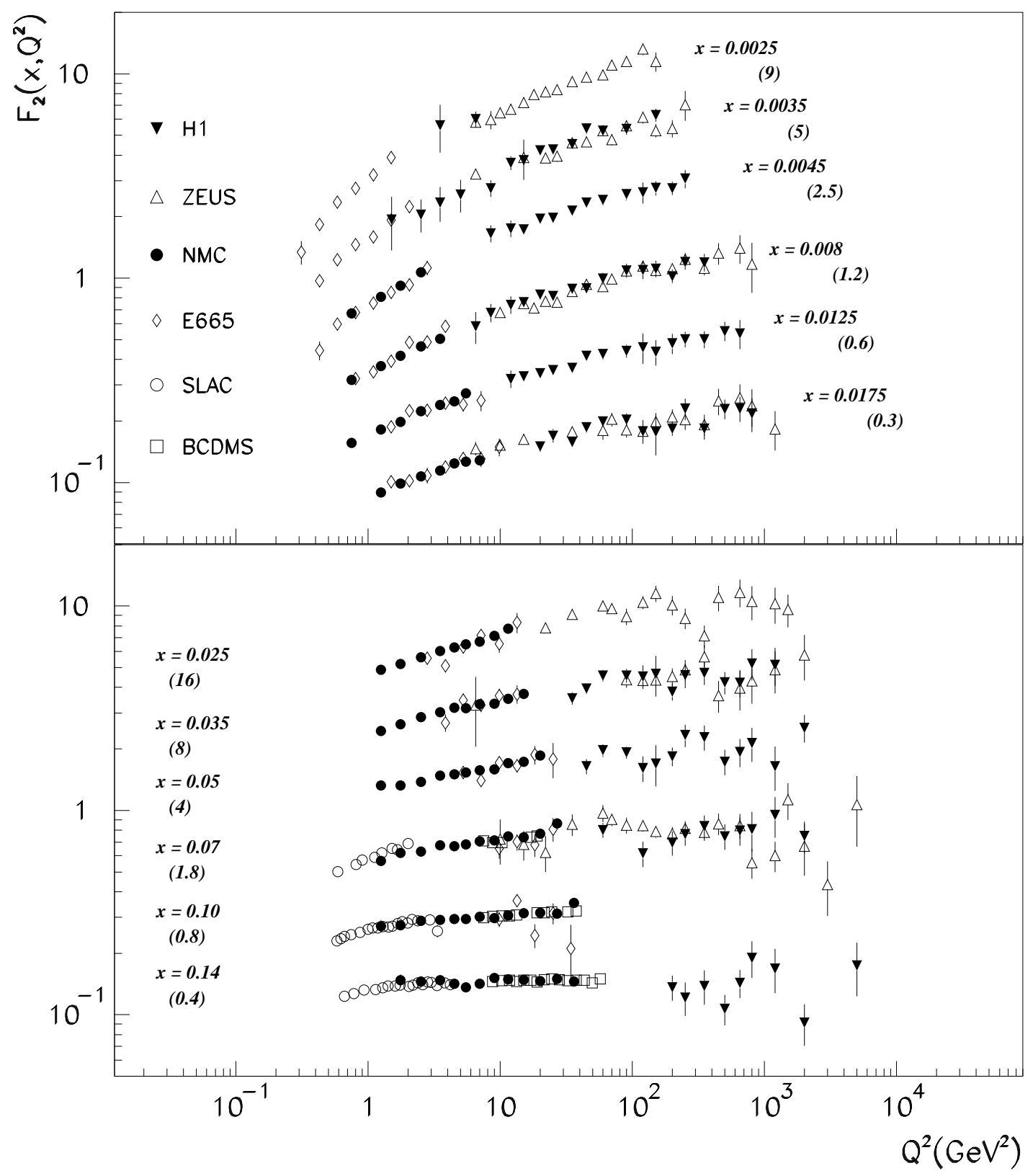

Fig. 30. $F_{2}^{p}$ data from HERA(94) and fixed target experiments at fixed $x$ as a function of $Q^{2}$. The data in each $x$ bin are scaled by the factors indicated in brackets. Here the NMC data are those from the $\mathrm{NMC}(95)$ dataset. 


\section{ZEUS 1995}

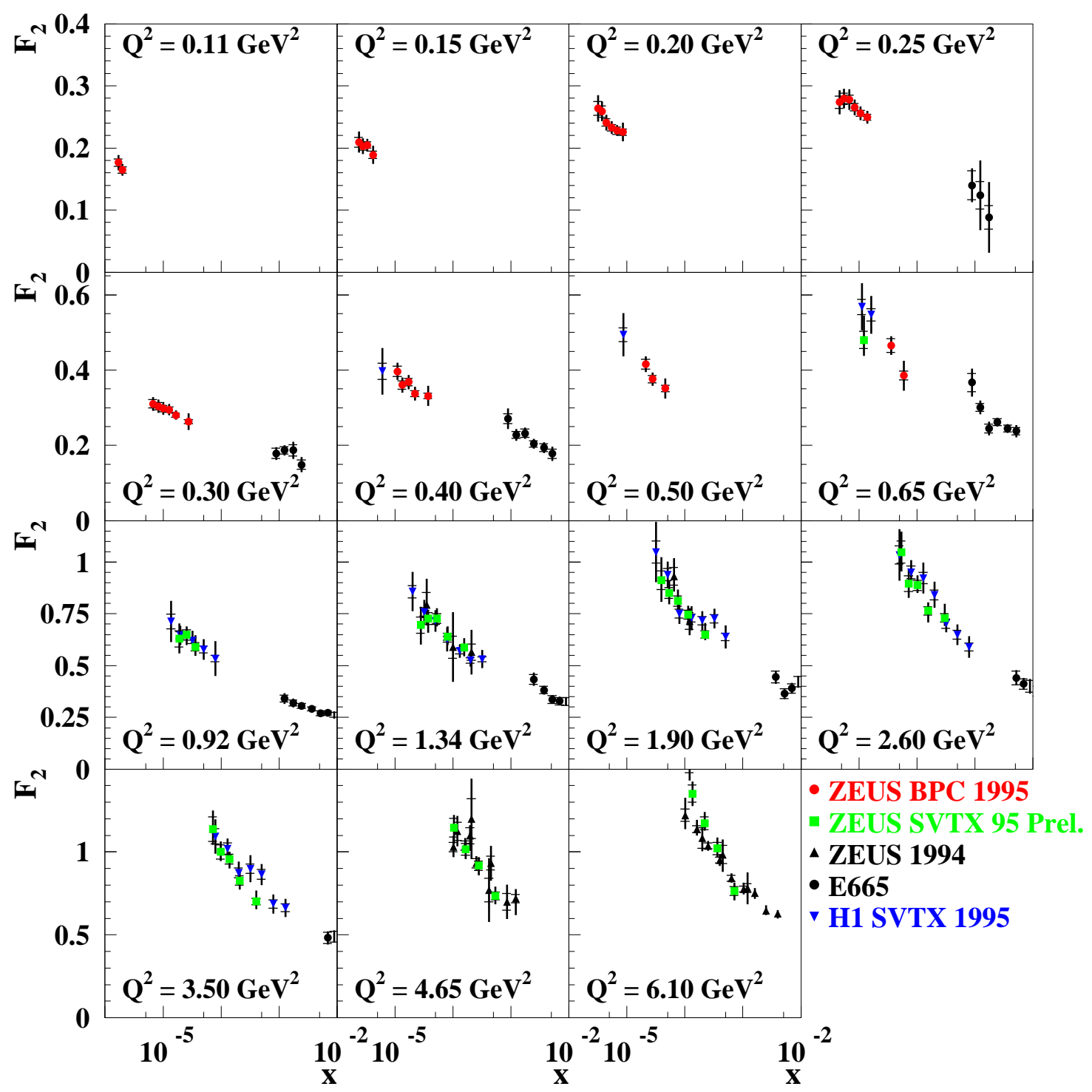

Fig. 31. $F_{2}^{p}$ at very small values of $Q^{2}$ from the E665, ZEUS(94), ZEUS(95)BPC, ZEUS(95)SVX (preliminary) and H1(95)SVX data sets 
the analysis has been reassessed and improved 106 to give $F_{2}$ and $x F_{3}$ values for $x$ between 0.0075 and 0.75 and $Q^{2}$ between 1.3 and $126 \mathrm{GeV}^{2}$ (CCFR(97) dataset). Improvements in the analysis include a new determination of the muon and hadron energy calibrations from test beam data and a more complete treatment of radiative corrections 107. Generally the estimates of systematic errors have been refined. Other corrections applied are: an isoscalar correction for the $6.8 \%$ excess of neutrons over protons in Fe; a correction for the charm quark threshold using the slowrescaling model with $m_{c}=1.34 \mathrm{GeV}$ and a correction for the mass of the $W$-boson. The $R_{S L A C}$ parametrization was used and nuclear effects have not been corrected for. The largest sources of systematic errors are the $1 \%$ errors in the muon and hadron energy scales. The error in the ratio $\sigma^{\bar{\nu}} / \sigma^{\nu}$ is also important. The world average value of the ratio from $\nu \mathrm{Fe}$ DIS experiments of $0.499 \pm 0.007$ was used. Other sources of systematics include the flux determination and variations in the physics models used in the Monte Carlo simulation. The structure function data are shown in Fig. 32.

As we have noted in Sec. 3.1 one expects $F_{2}^{\ell N} \approx \frac{5}{18} F_{2}^{\nu N}$ and this was a very important and early success of the QPM. CCFR have made a more exact comparison by using $F_{2}^{\ell N}=\frac{5}{18}\left(1-\frac{3}{5} \frac{s+\bar{s}-c-\bar{c}}{q+\bar{q}}\right) F_{2}^{\nu N}$ with the strange sea extracted from their own dimuon data 108 . The comparison is made with deuterium data from SLAC, NMC and BCDMS corrected to Fe using the $F_{2}^{\ell F e} / F_{2}^{\ell d}$ ratio as measured by SLAC and NMC, and is shown in Fig. 33 . The $F_{2}$ values generally agree well except in the low $x$ bin (0.0125) in which the CCFR values lie about $15 \%$ above those of NMC. The discrepancy is larger than the quoted systematic errors of the two experiments. It cannot be explained by increasing the size of the strange sea as this is limited by CCFR dimuon data, however it has been suggested that its distribution may be more complicated than usually assumed 100. Further possibilities are that the nuclear corrections are different for neutrinos and charged leptons or that the treatment of the charm threshold in the CCFR analyses 110 is not fully correct to NLO 111. Recently Caldwell 112 has compared the CCFR $F_{2}$ data, corrected to single protons, with that from ZEUS and NMC and concluded that the trend of the CCFR data at small $x$ may be in better agreement with the HERA data than that from NMC. It is possible that the HERMES experiment at HERA could measure $F_{2}$ in this overlap region and help to resolve the discrepancy.

The IHEP-JINR neutrino detector collaboration have recently published data on $F_{2}$ and $x F_{3}$ from three exposures of their detector to the wide band $\nu(\bar{\nu})$ beams at the Serpukhov U70 accelerator 13 . Events were selected to satisfy $W^{2}>1.7 \mathrm{GeV}^{2}$ and $6<E_{\nu(\bar{\nu})}<28 \mathrm{GeV}$. Samples of $741 \bar{\nu}$ events with $\left\langle Q^{2}\right\rangle=1.2 \mathrm{GeV}^{2}$ and $5987 \nu$ events with $\left\langle Q^{2}\right\rangle=2.3 \mathrm{GeV}^{2}$. Structure functions have been extracted in 6 bins with $0.052<x<0.563$ and $0.55<Q^{2}<4.0 \mathrm{GeV}^{2}$ with $R=0$ and $R=0.1$. Corrections have been made for acceptance, Fermi motion smearing, radiative effects and target non-isoscalarity. The overall normalization errors from the neutrino flux determination are $4 \%$ and $11 \%$ for $F_{2}$ and $x F_{3}$ respectively. 

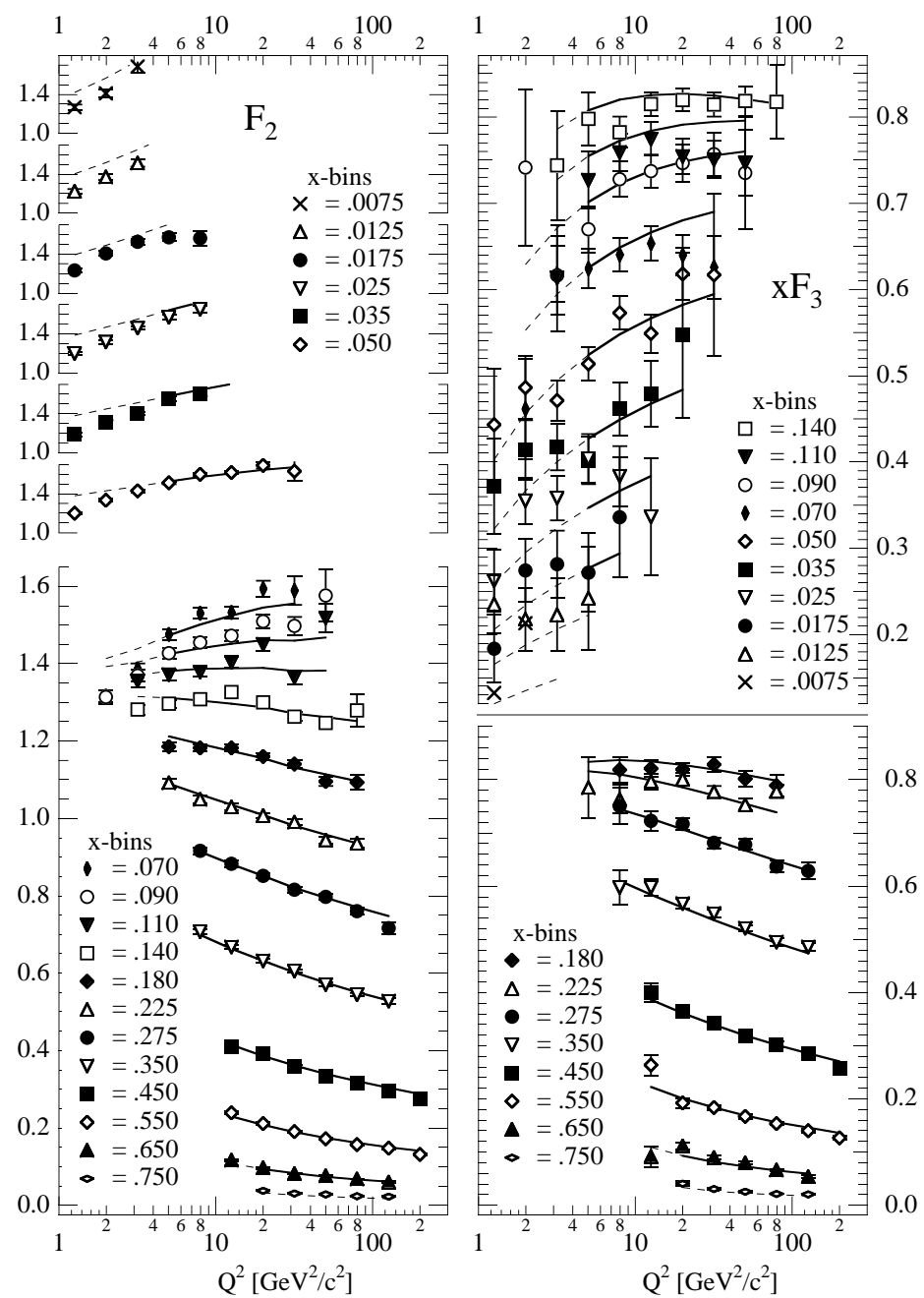

Fig. 32. $\operatorname{CCFR}(97) F_{2}$ and $x F_{3}$ data as functions of $Q^{2}$ at fixed $x$. The results of a NLO QCD are also shown (full line), the dashed line is the extrapolation of the fit to lower $Q^{2}$. 


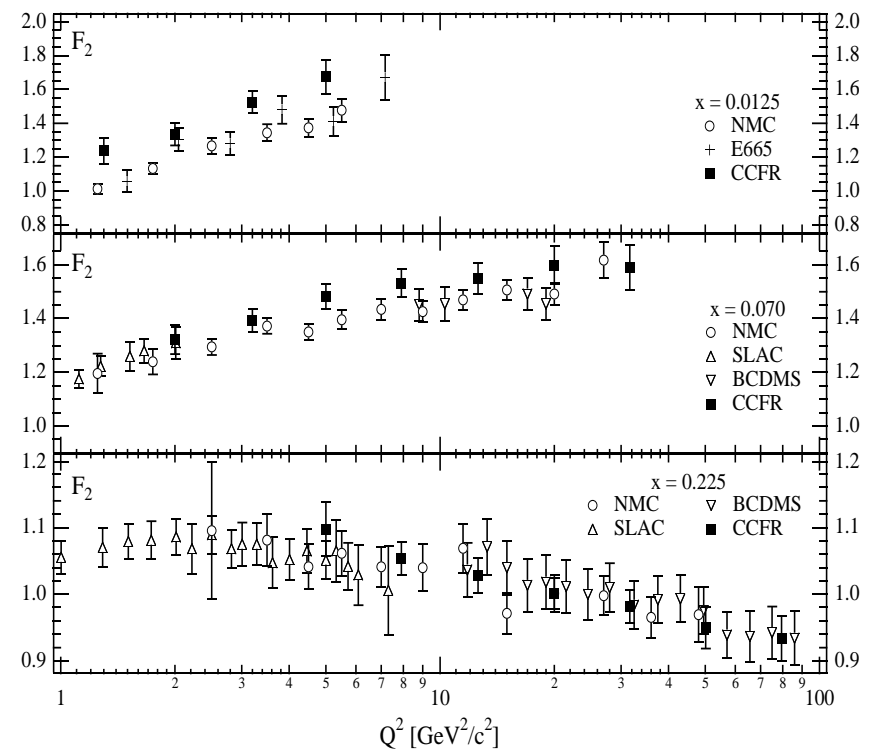

Fig. 33. Comparisons of $\operatorname{CCFR}(97) F_{2}$ values for $\nu F e$ with those for $\ell D$ from SLAC, NMC(97) and BCDMS converted using the ' $5 / 18$ ' relation as described in the text).

Given the size of the CCFR data sample it remains the definitive neutrino nucleon DIS experiment.

\subsection{Data on $R$}

In this section we will discuss the methods that have been used to measure $R$ or $F_{L}$ and summarise the existing data. Consider charged lepton induced NC scattering. It is convenient to write the double differential cross-section in terms of the cross-sections for the scattering of transverse and longitudinal virtual photons $\theta$

$$
\frac{d^{2} \sigma}{d x d Q^{2}}=\Gamma\left[\sigma_{T}\left(x, Q^{2}\right)+\varepsilon \sigma_{L}\left(x, Q^{2}\right)\right]
$$

where the virtual photon cross-sections are related to $F_{2}$ and $F_{L}$ by

$$
4 \pi^{2} \alpha F_{2}=\frac{\nu Q^{2} K}{\left(\nu^{2}+Q^{2}\right)}\left(\sigma_{T}+\sigma_{L}\right) \quad 4 \pi^{2} \alpha F_{L}=\frac{\nu Q^{2} K}{\nu^{2}} \sigma_{L}
$$

and

$$
\Gamma=\frac{\alpha}{\pi} \frac{M_{N} K}{s^{2} x^{2}(1-\varepsilon)}, \quad \varepsilon^{-1}=1+2\left(1+\frac{\nu^{2}}{Q^{2}}\right)\left[\frac{s\left(s-2 M_{N} \nu\right)}{M_{N}^{2} Q^{2}}-1\right]^{-1}
$$

with $\nu=Q^{2} /\left(2 M_{N} x\right)$. In these expressions the factor $K$ gives the flux of virtual photons which may be defined according to the conventions of Gilman 115 ( $K=$ 
$\left.\sqrt{\nu^{2}+Q^{2}}\right)$ or Hand114 $(K=\nu(1-x))$ such that it should be equal to that of a real photon beam $(K \rightarrow \nu)$ as $Q^{2} \rightarrow 0$. At small $x$ and/or large $Q^{2}$ we may drop terms in $Q^{2} / \nu^{2}\left(=4 M_{N}^{2} x^{2} / Q^{2}\right)$ and the above expressions simplify greatly. In particular,

$$
\varepsilon=\frac{2(1-y)}{1+(1-y)^{2}}
$$

and

$$
4 \pi^{2} \alpha F_{2}=Q^{2}\left(\sigma_{T}+\sigma_{L}\right) \quad 4 \pi^{2} \alpha F_{L}=Q^{2} \sigma_{L}
$$

At this level of approximation one may rewrite Eqs. $122-124$ to read

$$
\frac{d^{2} \sigma}{d x d Q^{2}}=\frac{2 \pi \alpha^{2}}{Q^{4} x} Y_{+}\left[\frac{1+\varepsilon R}{1+R}\right] F_{2}\left(x, Q^{2}\right),
$$

To measure $R$ directly at a fixed value of $\left(x, Q^{2}\right)$ requires data at two different values of $y$ and hence at two different centre of mass energies (because of the constraint $Q^{2}=s x y$ ), then

$$
R=\frac{\tilde{\sigma_{2}} Y_{+1}-\tilde{\sigma_{1}} Y_{+2}}{2 \tilde{\sigma_{1}}\left(1-y_{2}\right)-2 \tilde{\sigma_{2}}\left(1-y_{1}\right)}
$$

where $\tilde{\sigma}=\frac{Q^{4} x}{2 \pi \alpha^{2}} \frac{d^{2} \sigma}{d x d Q^{2}}$. This equation shows why $R$ is difficult to measure accurately, not only does one need very small statistical errors on $\tilde{\sigma}_{i}$ but also very good control of the systematic errors at the two different energies. From the form of the expression for $\varepsilon$ as a function of $y$ it can be seen that one gets the largest variation in $\varepsilon$, for a given change in $\sqrt{s}$, at large $y$. This condition is often difficult to achieve, particularly at HERA. If one has data at many different energies then $\sigma_{L}$ can be extracted from the slope of the total cross-section as a function of $\varepsilon-$ the 'Rosenbluth plot' of low energy nuclear and nucleon-electron scaftering.

Early fixed target measurements of $R$ were made by EMC 116, BCDMS 91 , CDHSW 11 and SLAC following the re-analysis of the data by Whitlow et al 68 . The most extensive results are those of SLAC, $R^{p}$ and $R^{d}$ measured for $0.1 \leq x \leq 0.9$, $0.6 \leq Q^{2} \leq 20 \mathrm{GeV}^{2}$, and BCDMS, $R^{p}$ and $R^{d}$ measured for $0.07 \leq x \leq 0.65$, $15 \leq Q^{2} \leq 50 \mathrm{GeV}^{2}$. The data satisfied $R^{p}=R^{d}$ but the behaviour of $R$ as a function of $x$ and $Q^{2}$ did not follow any model or theory, including pQCD. The need, none the less, for a good description of the behaviour of $R$ led the authors of ref. 68 to produce a convenient parametrization of their own data and data from EMC, BCDMS and CDHSW. It is

$$
R_{S L A C}=\frac{b_{1}}{\ln \left(Q^{2} / \Lambda^{2}\right)} \Theta\left(x, Q^{2}\right)+\frac{b_{2}}{Q^{2}}+\frac{b_{3}}{Q^{4}+(0.3)^{2}}
$$

where $\Theta\left(x, Q^{2}\right)=1+12\left(\frac{Q^{2}}{Q^{2}+10 .}\right)\left(\frac{(0.125)^{2}}{(0.125)^{2}+x^{2}}\right), \Lambda=0.2 \mathrm{GeV}$ and the best fit results for the parameters $b_{i}$ ( $\chi^{2}$ of 110 on 139 data points) are $b_{1}=0.06347, b_{2}=$ $0.57468, b_{3}=-0.35342 . R_{S L A C}$ is not valid for $Q^{2}<0.35 \mathrm{GeV}^{2}$. 
More recently $R$ has been measured by a new SLAC experiment 118 , by CCFR 119 and NMC120.95. The new data from SLAC are for hydrogen, deuterium and beryllium targets and cover the ranges $0.1 \leq x \leq 0.7,0.5 \leq Q^{2} \leq 7 \mathrm{GeV}^{2}$. The advantage of the new SLAC data is that $R$ is extracted from a single experiment which leads to a reduction of the systematic error from relative normalizations. The total systematic error is at the level of $10 \%$.

The CCFR $R$ measurements are from the same data sample discussed in Sec. 5.2 above. $R$ is extracted from a linear fit to the averaged cross-section data for $\nu F e$ and $\bar{\nu} F e$ versus $\varepsilon$. The measurements cover the ranges $0.01<x<0.6,4<Q^{2}<$ $300 \mathrm{GeV}^{2}$. The method assumes that $x F_{3}^{\nu}=x F_{3}^{\bar{\nu}}$ which is not exact at low $x$. Corrections are made for the differences caused by the strange sea and charm mass effects (see Eqs. 40, 41). The CCFR data, together with some of the earlier $R$ data are shown in Fig. 34. To describe the $R$ data at both low and high $Q^{2}$ Bodek, Rock and Yang 121 have developed a model which includes non-pQCD effects such as higher twist terms and target mass effects at low $Q^{2}$ but which matches smoothly to the next-to-lowest order expression for $R$ (worked out to order $\alpha_{s}^{2}$ in the coefficient functions 122) at large $Q^{2}$. The results of this model using various different parton distribution functions as input are shown in the figure. The dependence on the choice of PDF is not strong and the model gives a reasonable description of all the $R$ data.

The NMC results on $R$ come from the T1 trigger data sample already published and the more recent data from the small angle trigger T2. The data on $R^{p}$ and $R^{d}$ cover the ranges $0.002<x<0.12$ and $\left\langle Q^{2}\right\rangle=1.4-20.6 \mathrm{GeV}^{2}$. The largest source of systematic error is normalization uncertainty. The total systematic errors are 1-5 to 3 times larger than the statistical errors. This is shown Fig. 35(a), in which the inner error bar is statistical and the outer the sum of statistical and systematic errors in quadrature. The data are in good agreement with other measurements at large $x$ and provide a considerable increase in data for $x<0.1$, where $R$ is seen to rise as one would expect from its sensitivity to the gluon distribution function. This can be seen in Fig. 35(b).

At HERA no direct measurements of $R$ have yet been attempted as the collider has operated essentially at a fixed $\mathrm{CM}$ energy of around $300 \mathrm{GeV}$. In the meantime the H1 collaboration 101 have estimated $R$ by using their high statistics 1994 data and assuming that $F_{2}$ is well described by NLO QCD. As we have noted a number of times the contribution of $F_{L}$ to the double differential cross-section is suppressed by the factor $y^{2}$. The idea of the $\mathrm{H} 1$ method is first to determine $F_{2}$ by applying a NLO QCD fit to data satisfying $y<0.35$, i.e. in a region where the effect of $F_{L}$ in the cross-section is small. The fit is then extrapolated to the high $y$ region and used to subtract the contribution of $F_{2}$ from the cross-section at $y=0.7$, leading to an estimate of $R$. Although it has been checked that extrapolations based on some other models 123.24 give the same result at $y=0.7$ within a few per cent, the extrapolation remains the most uncertain part of this analysis. ${ }^{1}$ The

\footnotetext{
1 Thorne 125 has argued in some detail that the extrapolation error may be considerably larger.
} 

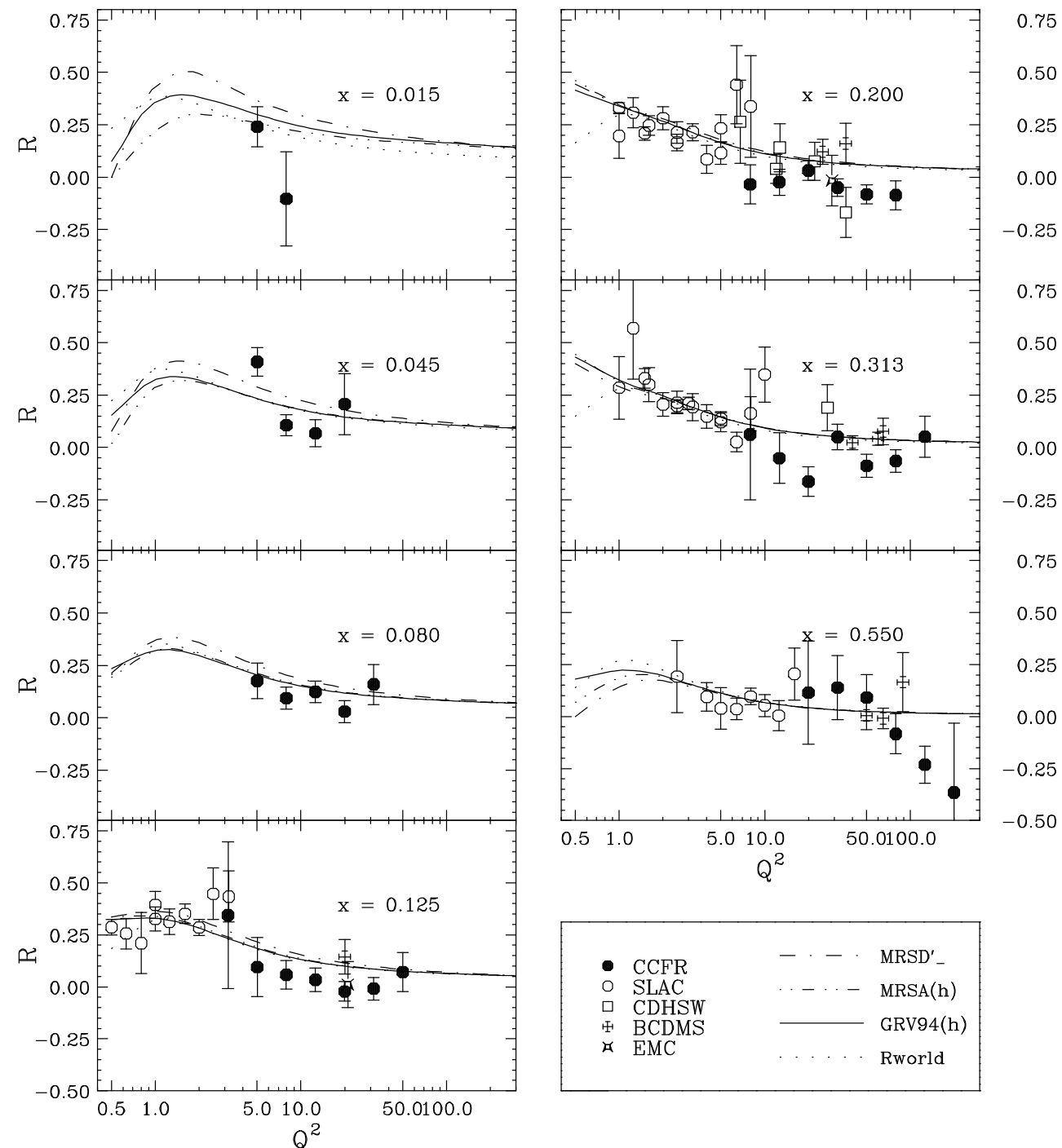

Fig. 34. $R$ at fixed $x$ vs $Q^{2}$. Data from CCFR is shown together data from EMC, CDHSW, BCDMS and SLAC. The curves are from Bodek, Rock and Yang 21 (see text). 

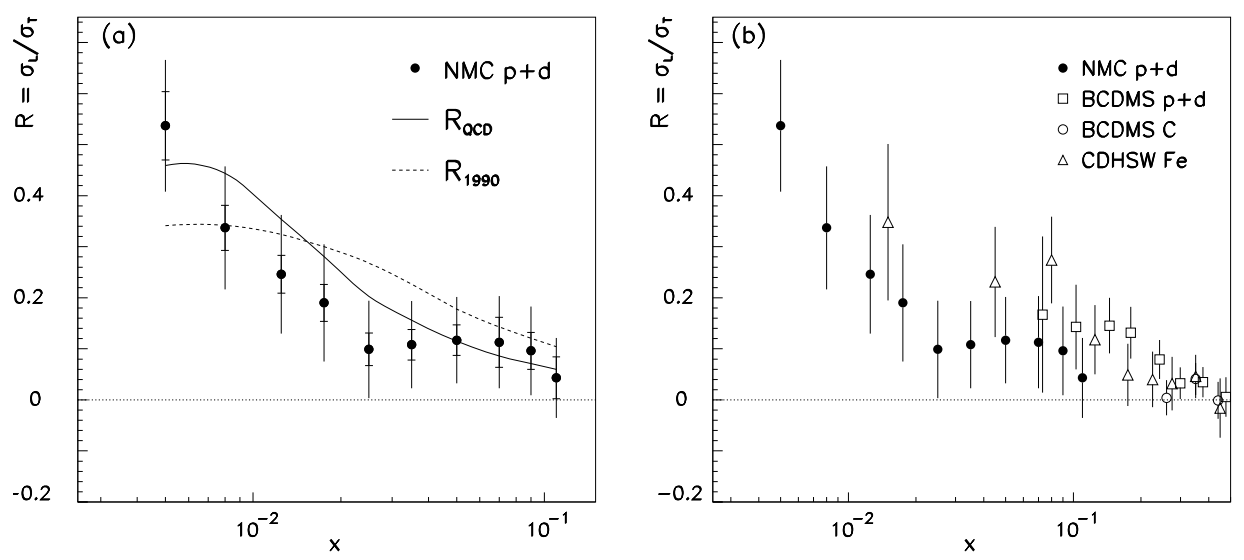

Fig. 35. Left: $R$ vs $x$, data from NMC. The full curve is from a QCD fit and the dotted curve is from $R_{S L A C}$. Right: data on $R$ from NMC, BCDMS and CDHSW.

data sample for this study is essentially that of the 'nominal vertex' sample H1(94) but with the minimum cut on the energy of the scattered positron lowered from 11 to $6.5 \mathrm{GeV}$, thus allowing higher values of $y$ to be reached. The data cover the ranges $0.6<y<0.78$ and $7.5<Q^{2}<42 \mathrm{GeV}^{2}$. A careful study of radiative corrections was made using the HECTOR code and they are stable within about half the statistical error of the cross-sections. Systematic errors are highly correlated, sources studied are $y$ dependent effects such as cut values and the photoproduction background subtraction, the relative uncertainty in the luminosities and the error from the subtraction of the fitted $F_{2}$. Averaging over all the data gives the result $F_{L}=0.52 \pm 0.03(\text { stat })_{-0.22}^{+0.25}($ sys $)$ for $\left\langle Q^{2}\right\rangle=15.4 \mathrm{GeV}^{2}$ and $\langle x\rangle=2.43 \times 10^{-4}$, well compatible with $\mathrm{pQCD}$ calculations using recent parton distribution functions. The result is shown in Fig. 36 .

An alternative approach to the measurement of $R$ at HERA has been proposed by Krasny, Placzek and Spiesberger 126. The idea of the method is to use hard initial state radiation as a way of varying the incident $\mathrm{e}^{ \pm}$beam energy thus allowing the cross-section to be measured for different values of $\varepsilon$, but fixed $\left(x, Q^{2}\right)$ - rather analogous to a broad-band neutrino beam. Experimentally one uses the luminosity monitor to measure the energy of the hard ISR photon, $E_{\gamma}$. Integrating over photon emissions angles inside a cone $\theta_{\gamma} \geq \pi-\theta_{a}$ and neglecting infrared and collinear finite terms, the cross-section for $e p \rightarrow e \gamma X$ is

$$
\frac{d^{3}}{d x d Q^{2} d z}=\alpha^{3} P(z) \frac{1+(1-y(z))^{2}}{x Q^{4}}\left[F_{2}\left(x, Q^{2}\right)-(1-\varepsilon(z)) F_{L}\left(x, Q^{2}\right)\right],
$$




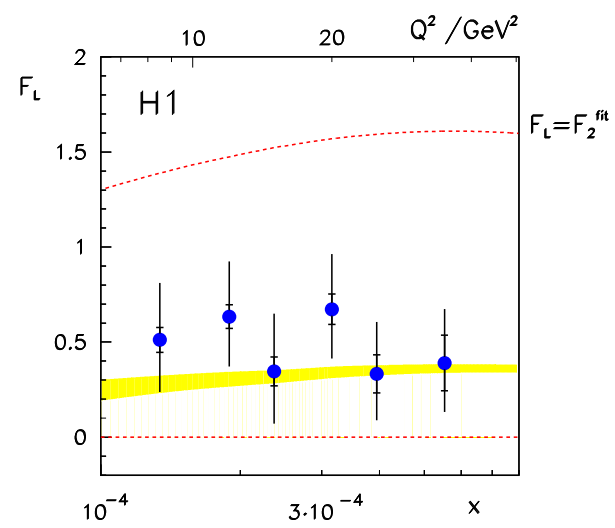

Fig. 36. Measured longitudinal structure function $F_{L}$ by $\mathrm{H} 1$ for $y=0.7$ as functions of $x$ and $Q^{2}$ for $Q^{2}=8.5,12,15,20,25$ and $35 \mathrm{GeV}^{2}$. The error band represents the uncertainty of the calculation of $F_{L}$ using the gluon and quark distributions, as determined from the NLO QCD analysis of the $\mathrm{H} 1$ data for $y \leq 0.35$ and the BCDMS data. The dashed lines define the allowed range of $F_{L}$ values from $F_{L}=0$ to $F_{L}=F_{2}$ where $F_{2}$ is given by the QCD fit.

where $P(z)$ is the function

$$
P(z)=\frac{1+z^{2}}{1-z} \ln \left(\frac{E_{e}^{2} \theta_{a}^{2}}{m_{e}^{2}}\right)-\frac{z}{1-z}
$$

and

$$
\varepsilon(z)=\frac{2(1-y(z))}{1+(1-y(z))^{2}} \quad y(z)=\frac{Q^{2}}{x z s}
$$

where $z=\left(E_{e}-E_{\gamma}\right) / E_{e}$. The variables $x$ and $Q^{2}$ are the true values and if the electron method is used for their reconstruction the formulae given in Eq. 118 must be used to take account of the radiated energy. $F_{L}$ or $R$ is extracted from the dependence of the cross-section on $\varepsilon$. A measurement of $F_{L}$ for $0.0006<x<0.02$, $15<Q^{2}<120 \mathrm{GeV}^{2}$ at HERA could be made using ISR events, but would need a total luminosity of the order of $200 \mathrm{pb}^{-1}$.

The method is a very elegant one in principle, but it has not been possible to exploit it yet in practice. The major problems are the small cross-section, the stringent requirements on resolution of the kinematic variables (particularly $x$ ) and control of the bremsstrahlung background. Improvements have been suggested by by Frey 127, using a more restricted kinematic range, and by Favart et al 128, using a modified analysis method. The latter exploits the knowledge of $F_{2}$ and extracts $R$ from its influence on the shape of the $\varepsilon$ distribution of events. However the method does also require a very low value of $E_{\text {min }}^{\prime}$, well below $5 \mathrm{GeV}$ and, if possible, as small as $2 \mathrm{GeV}$. Although none of the direct experimental methods is likely to give a measurement of $R$ to better that $20-30 \%$, it is important to have a direct check 
on this quantity at the high values of $W$ and small values of $x$ available at HERA. The uncertainty in $R$ could eventually be one the limiting systematic errors on the extraction of $F_{2}$ at low $x . F_{L}$ is also a very interesting quantity in its own right, because of its very direct dependence on the gluon density as shown by Eq. 83 .

Recently the possibility of measuring $R$ at HERA by reducing the beam energies has been studied 129. The conclusion was that $R$ could be measured with a precision of about $15-20 \%$ at low $x$ and $Q^{2}<100 \mathrm{GeV}^{2}$, by running at proton beam energies of 450,350 and $250 \mathrm{GeV}$. A data sample of $10 \mathrm{pb}^{-1}$ would be required at each energy. One lower beam energy data sample would be sufficient to give a measurement with approximately $30 \%$ precision in a large part of this kinematic region.

\subsection{Open charm production and $F_{2}^{c \bar{c}}$}

Heavy quark production in DIS is thought to occur predominantly through the boson-gluon fusion process (BGF) - see Fig. 13(a) - at relatively small $x$. Other mechanisms such as diffractive production (which is important for $J / \psi$ production) have much smaller cross-sections. The evidence for intrinsic charm in the proton is somewhat equivocal and in any case such events would be produced at large $x$.

The first measurement of $F_{2}^{c \bar{c}}$ was made by the BFP collaboration 130 who used a $209 \mathrm{GeV}$ muon beam at FermiLab and a multimuon spectrometer to extract $F_{2}^{c \bar{c}}$ from dimuon events over a range $0.0013<x<0.13,0.63<Q^{2}<63 \mathrm{GeV}^{2}$. Later the EMC collaboration 131 measured $F_{2}^{c \bar{c}}$, in the range $0.0042<x<0.422$, $1<Q^{2}<70 \mathrm{GeV}^{2}$, from di- and trimuon events produced from $250 \mathrm{GeV}$ muon scattering on an iron target. The dimuon data were corrected for acceptance effects using the lowest order BGF model of Leveille and Weiler 11 and a mean semileptonic branching ratio for the $D$ meson to muons of $8.2 \%$ was used to correct to the $c \bar{c}$ cross-section. The results from both experiments were found to be well represented by the BGF production mechanism. However because of the rather low $\mathrm{cm}$ energies of around $20 \mathrm{GeV}$, their results could not rule out other explanations.

The advent of HERA, with an order of magnitude increase in $W$ and a reach to much smaller $x$ values, together with the sophisticated detectors H1 and ZEUS capable of measuring many details of the DIS final state gives the prospect of accurate data on $F_{2}^{c \bar{c}}$ over a much wider range of $x$ and $Q^{2}$. The second major step forward since the early measurements is the calculation of the massive quark coefficient functions to order $\alpha_{s}^{2}$ by Laenen et al 12132 and the attempts to provide consistent treatments of heavy quark production from the threshold region $Q^{2} \sim m_{q}^{2}$ to the asymptotic region $Q^{2} \gg m_{q}^{2}$ by MRRS 45 and CTEQ 4 - see Sec. 3.6. In addition the differential distributions for the rapidity and transverse momentum of the charm quark have also been worked out to next-to-lowest order 133.

Both the H1 and ZEUS experiments have searched for $D^{*}$ production in DIS using the well-established technique which exploits the accurately known mass difference $\Delta M=M\left(K \pi \pi_{s}\right)-M(K \pi)^{\mathrm{m}}$ (from the decay chain $D^{*+} \rightarrow D^{0} \pi_{s}^{+} \rightarrow$ $\left.K^{-} \pi^{+} \pi_{s}^{+}\right)$as the primary signal. The $D^{*}$ is only just above threshold and its decay

$\bar{m}$ The PDG value is $145.42 \pm 0.05 \mathrm{MeV}$. 
produces a 'slow' pion labelled as $\pi_{s}$. Although the signal is a very clean one, the combined branching ratio for the $D^{*} \rightarrow K \pi \pi$ decay is only $2.62 \pm 0.10 \% 134$, which means that this measurement requires high luminosity.

The H1 collaboration 135 has made a detailed study of DIS charm production using both $D^{*}$ and $D^{0}$ channels. The analysis is based on $2.97 \mathrm{pb}^{-1}$ of 1994 DIS events, restricted to $10<Q^{2}<100 \mathrm{GeV}^{2}$ and $y<0.53$. Charged particles were reconstructed in the $\mathrm{H} 1$ central tracker and must have at least $0.25 \mathrm{GeV}$ momentum transverse to the beam line. Particle identification is not used but charged particle are ranked in order of the magnitude of their momentum in the $\gamma^{*} p$ frame, to take advantage of the fact that the fragmentation function for charmed mesons is 'harder' than that for the light mesons and so the decay products will have large momenta. Using only highly ranked particles to construct the $D^{0}$ reduces the combinatorial background. Charm events were selected by using the $D^{*} \rightarrow D^{0} \pi$ ( $\Delta M$ method) and also a direct inclusive $D^{0} \rightarrow K^{-} \pi^{+}$search. In both analyses the $D^{0}$ candidate was required to satisfy $\left|\eta\left(D^{0}\right)\right|<1.5^{\mathrm{n}}$. The $D^{*}$ and $D^{0}$ signals are extracted by fitting the $\Delta M$ and $K \pi$ mass distributions, respectively, with Gaussians and suitably shaped background functions. The resulting yields of signal events are $103 \pm 13 D^{*}$ events and $144 \pm 19$ events for the inclusive $D^{0}$ analysis, with $20 \pm 5$ events in common. The data are corrected for acceptance and resolution effects, using the AROMA Monte Carlo 136, to the full phase space in $p_{T}(D)$ and $\eta(D)$ and the region $10<Q^{2}<100 \mathrm{GeV}^{2}, y<0.7$. The efficiencies for reconstructing $D^{*}$ and $D^{0}$ mesons, including acceptance, are about $16 \%$ and $6 \%$ respectively. The cross-sections for $e p \rightarrow e D X$ and $e p \rightarrow e D^{*} X$ are $20.4 \pm 2.7_{-2.4-1.2}^{+2.7+1.6} \mathrm{nb}$ and $7.8 \pm 1.0_{-1.0}^{+1.2} \pm 0.6 \mathrm{nb}$ respectively, where the last error refers to model dependent uncertainties. The charm production mechanism has been studied by measuring the the $x_{D}=2\left|\mathbf{p}_{D}\right| / W$ distribution in the $\gamma^{*} p$ frame. The normalized $x_{D}$ distribution for an average $\gamma^{*} p \mathrm{CM}$ energy of $125 \mathrm{GeV}$ and $\left|\eta_{D}\right|<1.5$ is shown in Fig. 37 compared to calculations of the BGF mechanism using the AROMA Monte Carlo simulation and to the expectation from the charm sea only (using LEPTO/MEPS). From these comparisons, H1 has set an upper limit of 5\% (at 95\% CL) on charm production from the sea. A similar result has been found by the ZEUS collaboration from the DIS $D^{*}$ analysis described below.

To calculate the inclusive charm production cross-section one needs to know the probability that the charm quark will fragment into a $D^{0}$ or $D^{*}$ meson, $P(c \rightarrow D)$ and to correct for the small fraction, $\xi$, of indirect $D$ mesons produced through $B$ meson decay or fragmentation,

$$
\sigma(e p \rightarrow e c \bar{c} X)=\frac{1}{2} \frac{\sigma(e p \rightarrow e D X)}{P(c \rightarrow D)(1+\xi)}
$$

From measurements at $e^{+} e^{-}$colliders, $P\left(c \rightarrow D^{0}\right) \sim 50 \%, P\left(c \rightarrow D^{* \pm}\right) \sim 25 \%$ and $\xi$ is about $2 \%$. The final result averaging over both $D^{*}$ and $D^{0}$ channels is

\footnotetext{
$\mathrm{n}$ The pseudo-rapidity $\eta=-\ln (\tan (\theta / 2))$ where $\theta$ is the polar angle with respect to the proton beam direction.
} 


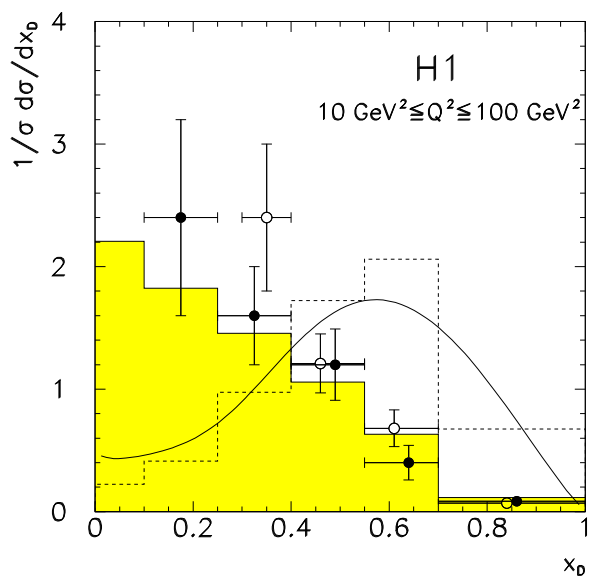

Fig. 37. Distribution of $x_{D}=2\left|\mathbf{p}_{D}\right| / W$ measured by H1 in the $\gamma^{*} p$ frame (full points $D^{*}$ data and open points $D^{0}$ data), compared to the BGF (shaded histogram) and charm sea (dashed histogram) expectations.

$\sigma(e p \rightarrow e c \bar{c} X)=17.4 \pm 1.6($ stat $) \pm 1.7($ sys $) \pm 1.4($ model $) \mathrm{nb}$, where the last error is from model dependent uncertainties in the extrapolation to the full phase space. The systematic error is dominated by the mass resolution and the separation of signal and background, other contributions come from tracker efficiency, luminosity measurement and radiative corrections. H1 has compared their measured crosssection with next-to-lowest order calculations using MRS and GRV gluons and $m_{c}$ between 1.3 and $1.7 \mathrm{GeV}$ and find that the calculated cross-sections are in the range $8.7-11.4 \mathrm{nb}$, well below the measured value. Using a gluon distribution from a NLO QCD fit to their own $1994 F_{2}$ data the $m_{c}=1.5, \mathrm{H} 1$ predict a value of $13.6 \pm 1.0 \mathrm{nb}$.

Assuming that $R=0, F_{2}^{c \bar{c}}$ is related to the $e p \rightarrow e c \bar{c} X$ cross-section by

$$
\frac{d^{2} \sigma(c \bar{c})}{d x d Q^{2}}=\frac{2 \pi \alpha^{2}}{Q^{4} x}\left(1+(1-y)^{2}\right) F_{2}^{c \bar{c}}\left(x, Q^{2}\right) .
$$

H1 has binned the $D^{*}$ and $D^{0}$ data into 9 bins with mean values of $x$ and $Q^{2}$ of $0.008,0.0020,0.0032$ and $12,25,45 \mathrm{GeV}^{2}$, respectively. The conversion to $c \bar{c}$ crosssections follows the procedure described above and $F_{2}^{c \bar{c}}$ is extracted using Eq. 134. The results are shown in Fig. 38. Averaging over all bins, $\mathrm{H} 1$ finds a value $0.237 \pm$ $0.021_{-0.039}^{+0.043}$ for the ratio $F_{2}^{c \bar{c}} / F_{2}$.

The ZEUS collaboration has also studied DIS $D^{*}$ production 137. DIS events from $2.95 \mathrm{pb}^{-1}$ of the 1994 sample satisfying $y<0.7,5<Q^{2}<100 \mathrm{GeV}^{2}$ were chosen. Charged particle tracks, reconstructed in the ZEUS central tracker, had to have a transverse momentum $p_{T}>0.125 \mathrm{GeV}$ and a polar angle satisfying $20^{\circ}<$ $\theta<160^{\circ}$. The $K \pi$ invariant mass from opposite sign particles had to lie in the 
range $1.4-2.5 \mathrm{GeV}$ and if adding a third track (with opposite sign to the $K$ track) gave $\Delta M<180 \mathrm{MeV}$, the three charged particles formed a $D^{*}$ candidate. $D^{*}$ candidates were required to satisfy $1.3<p_{T}\left(D^{*}\right)<9.0 \mathrm{GeV}$ and $\left|\eta\left(D^{*}\right)\right|<1.5$. Combinatorial background was subtracted using an average of estimates from sidebands in the $\Delta M$ distribution and that from wrong-sign particle combinations. In the given kinematic region $122 \pm 17$ signal events were found above a background of $95 \pm 8$. The corrections for detector effects and acceptance were calculated using the DIS heavy flavour production simulation programme AROMA. For the kinematical region selected by the cuts, the mean event selection efficiency is about $75 \%$ and mean $D^{*}$ reconstruction efficiency is about $38 \%$. The total systematic error on the cross-section is $15 \%$ with the major contributions coming from the signal selection and background subtraction, Monte Carlo corrections, radiative effects and the overall normalization.

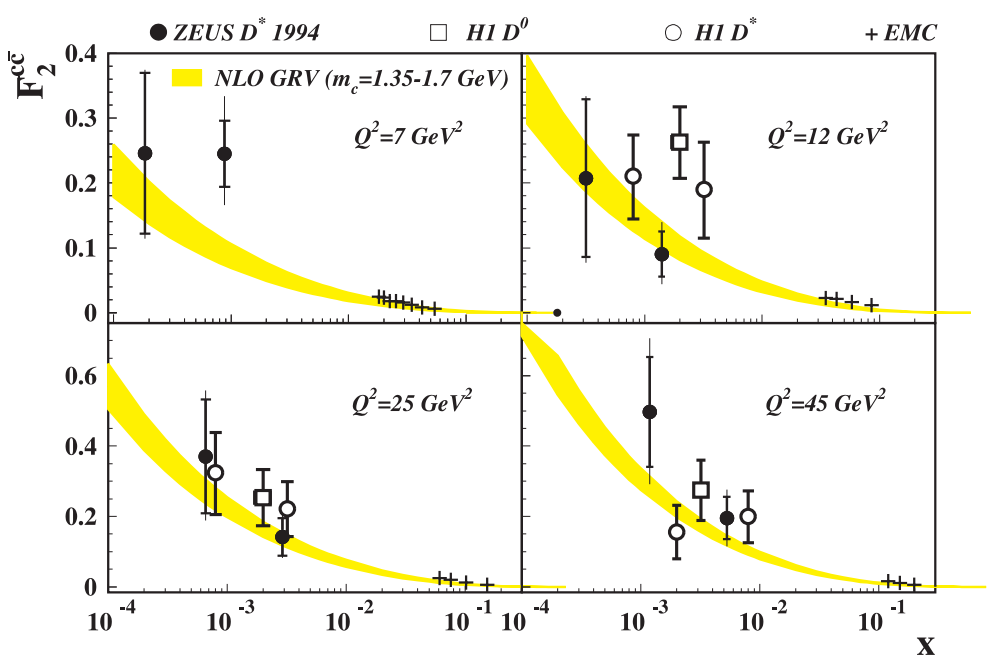

Fig. 38. $F_{2}^{c \bar{c}}$ from the ZEUS and H1 analyses of the HERA 1994 data. Earlier results from EMC are also shown. The shaded band corresponds to a next-to-lowest order QCD calculation using the GRV94 gluon distribution and a range of charm quark masses (upper edge $1.35 \mathrm{GeV}$, lower edge $1.7 \mathrm{GeV}$ ).

Integrating the cross-section over the region $5<Q^{2}<100 \mathrm{GeV}^{2}$ and $y<0.7$ ZEUS finds the result $\sigma\left(e p \rightarrow e D^{*} X\right)=5.3 \pm 1.0 \pm 0.8 \mathrm{nb}$ for $1.3<p_{T}<9.0 \mathrm{GeV}$ and $|\eta|<1.5$. Using the GRV94 gluon density and $m_{c}=1.5 \mathrm{GeV}$, the next-to-lowest order calculated cross-section(for the same restricted kinematic region) is $4.15 \mathrm{nb}$, about one sigma below the measured cross-section. ZEUS have followed a similar procedure to that described above for $\mathrm{H} 1$ to extrapolate the $D^{*}$ cross-section to the full phase space. The next-to-lowest order calculation of Harris and Smith 133 was used for this purpose and it is found that about $50-65 \%$ of the cross-section is outside the measured region. $F_{2}^{c \bar{c}}$ is extracted for two $x$ bins at each of four values of 
$Q^{2}$ and the results are shown in Fig. 38 together with those from $\mathrm{H} 1$ and EMC This figure also shows a calculation from the GRV94 parton distribution functions 39 for a range of charm quark masses between 1.35 and $1.7 \mathrm{GeV}$. It should also be noted that the recent CTEQ4F3 44 or MRRS 4 gluon densities give results within $5 \%$ of the GRV94 parametrization

The first results from HERA on $F_{2}^{c \bar{c}}$ are very encouraging. The charm signal can be identified cleanly, backgrounds are under control and the measurements indicate that the BGF process is dominant. However high precision results await high luminosity from HERA, though the use of silicon micro-vertex detectors could improve the detection efficiency by an order of magnitude. For the future there is still much to do. Is the apparent discrepancy between next-to-lowest order calculation and the measured cross-section at low $Q^{2}$ real? Will better data help to choose between the various approaches to $c \bar{c}$ production and the quark versus parton question at large $Q^{2}$ ? Will precise data provide more constraints on the gluon and possibly shed light on the necessity or otherwise of terms beyond standard DGLAP in the QCD evolution equations (see Sec. 7.6)?

\section{5. $F_{2}^{d} / F_{2}^{p}$ and $R^{d}-R^{p}$}

The ratio $F_{2}^{d} / F_{2}^{p}$ is directly related to $F_{2}^{n} / F_{2}^{p}$ which gives information on the ratio of up and down quarks in the nucleon. Because the $Q^{2}$ dependences of $F_{2}^{d}$ and $F_{2}^{p}$ are similar one expects the ratio to be almost independent of $Q^{2}$, the slight residual dependence can be calculated from pQCD. Similarly the difference $R^{d}-R^{p}$ is expected to be small and is sensitive to differences in the gluon distributions in the proton and neutron.

The NMC collaboration has recently published 138 accurate and extensive measurements of the ratio $F_{2}^{d} / F_{2}^{p}$ and the difference $R^{d}-R^{p}$. The ratio data cover the range $0.001<x<0.8$ and $0.1<Q^{2}<145 \mathrm{GeV}^{2}$. They are derived from the previously mentioned large and small angle trigger data 93,95 and an additional small angle trigger T14. T14 uses only the central part of the muon beam and is derived from small scintillators just above and below the muon beam. Measurement of the ratio exploits the special feature of the NMC target arrangement (see Sec. 4.1.1) which has two pairs of targets with $\mathrm{H}_{2}$ and $\mathrm{D}_{2}$ up and down stream, respectively, in one and with the order reversed in the other. By alternating the target pairs in the beam, the measured cross-section ratio does not depend on either the incident muon flux or the detector acceptance. The systematic errors can thus be kept to a minimum and the region in which the ratio is measured is larger than that for $F_{2}^{p}$ or $F_{2}^{d}$ alone.

If $R_{d}=R_{p}$ then $F_{2}^{d} / F_{2}^{p}$ is given by the cross-section ratio $\sigma^{d} / \sigma^{p}$ in a given $\left(x, Q^{2}\right)$ bin - see Eq. 11. Given that the difference $\Delta R=R^{d}-R^{p}$ is small, the group use the following approximate relation

$$
\frac{\sigma^{d}}{\sigma^{p}} \approx \frac{F_{2}^{d}}{F_{2}^{p}}\left(1-\frac{1-\varepsilon}{(1+\bar{R})(1+\varepsilon \bar{R})} \Delta R\right)
$$


where $\bar{R}=\left(R^{d}+R^{p}\right) / 2$. The results are extracted in two steps. First $\Delta R$ averaged over $Q^{2}$ is extracted for each $x$-bin by fitting the data in a bin to Eq. 135 using 4 parameters $\left(\Delta R, \bar{R}, a_{1}, a_{2}\right)$ where $F_{2}^{d} / F_{2}^{p}=a_{1}+a_{2} \ln Q^{2}$. The values of $\Delta R$ are all small and $\Delta R$ shows no significant $x$ dependence. Averaging over $x$ gives $\Delta R=0.004 \pm 0.012$ (stat) \pm 0.011 (sys) at an average $Q^{2}$ of $5 \mathrm{GeV}^{2}$ and $0.003<$ $x<0.35$. Having established that $\Delta R$ is compatible with zero, the second step in the extraction of the structure function ratio data is to take $\Delta R$ as zero so that $F_{2}^{d} / F_{2}^{p}=\sigma^{d} / \sigma^{p}$. Neglecting nuclear effects in the deuteron $F_{2}^{n} / F_{2}^{p}=2 \sigma^{d} / \sigma^{p}-1$.

The E665139 collaboration collected data on the ratio $\sigma^{d} / \sigma^{p}$ in 1991. In addition to the small angle trigger (SAT) used for structure function measurements, they also collected data with the EM calorimeter trigger which allowed them to get to $Q^{2}$ as small as $0.001 \mathrm{GeV}^{2}$. The calorimeter trigger recognizes very low $Q^{2}$ inelastic scattering events by the large spread of energy deposits and in particular can reject elastic $\mu e$ events which are a significant background. The ratio $F_{2}^{n} / F_{2}^{p}$ is extracted from the cross-section ratio ignoring any nuclear effects. Overall systematic errors are in the range $2-3.5 \%$, depending on the trigger, dominated by uncertainty in the relative acceptances. No significant $Q^{2}$ dependence is found but at low $x$ the ratio is less than 1 , more precisely for $x \leq 0.01, F_{2}^{n} / F_{2}^{p}=0.935 \pm 0.008 \pm 0.034$ indicating that there may be some nuclear shadowing in this region.

The results for $F_{2}^{n} / F_{2}^{p}$ as a function of $x$ averaged over $Q^{2}$ are shown in Fig. 5.5 for both NMC and E665. One should be warned that the $F_{2}^{n} / F_{2}^{p}$ as defined above may deviate from the free nucleon ratio, though such effects are expected to be small for deuterium.

\subsection{Sum rule data}

We summarise fairly recent data on the Gottfried sum rule. Recent evaluations of the Gross Llewellyn-Smith sum rule will be discussed in Sec. 6.2 in the context of measurement of $\alpha_{S}$.

From the QPM relations given in Eqs. 14 and 15 and valence quark sum rules one finds

$$
S_{G}=\int_{0}^{1}\left(F_{2}^{\mu p}-F_{2}^{\mu n}\right) \frac{d x}{x}=\frac{1}{3}+\frac{2}{3} \int_{0}^{1}(\bar{u}-\bar{d}) d x .
$$

Early data supported the assumption that the $q \bar{q}$ sea was $\mathrm{SU}(2)$ symmetric, i.e. $\bar{u}=\bar{d}$. Using the relation

$$
F_{2}^{p}-F_{2}^{n}=2 F_{2}^{d} \frac{\left(1-F_{2}^{n} / F_{2}^{p}\right)}{\left(1+F_{2}^{n} / F_{2}^{p}\right)}=2 F_{2}^{d} \frac{\left(1-F_{2}^{d} / F_{2}^{p}\right)}{F_{2}^{d} / F_{2}^{p}}
$$

the integral can be evaluated by using ratio data and $F_{2}^{d}$. In 1991 NMC published an evaluation of the sum rule using their early data on the ratio $F_{2}^{d} / F_{2}^{p}$ at 90 and $280 \mathrm{GeV}$ muon beam momentum and $F_{2}^{d}$ from a global fit. This result showed that 


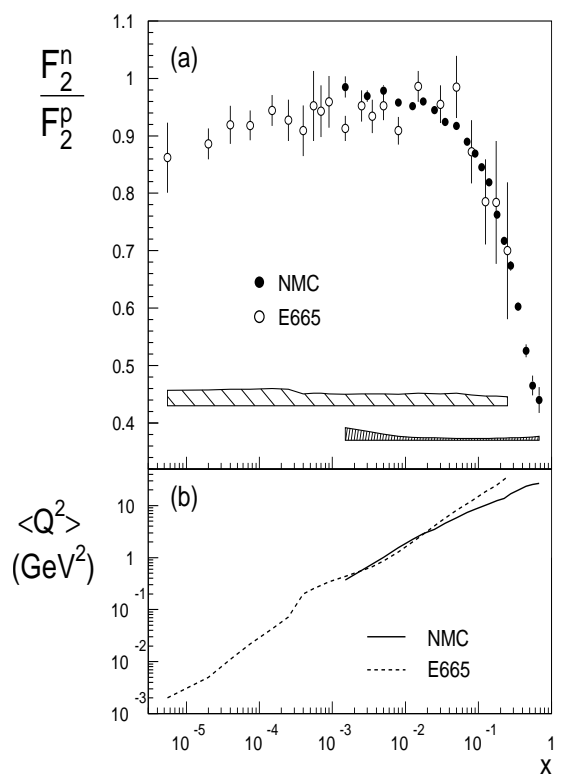

Fig. 39. $F_{2}^{n} / F_{2}^{p}$ averaged over $Q^{2}$ as a function of $x$ from the NMC and E665 experiments.

the integral was significantly below $1 / 3$ and that $\bar{u} \neq \bar{d}$. NMC made a more exact evaluation at $Q^{2}=4 \mathrm{GeV}^{2}$ using their own $F_{2}^{d}$ data in 1994140 giving

$$
S_{G}(0.004-0.8)=0.221 \pm 0.008(\text { stat. }) \pm 0.019 \text { (sys.). }
$$

The final NMC data referred to in the previous section gives $0.2281 \pm 0.0065$ consistent with the 1994 result. To complete the integral, the contributions from the unmeasured regions at high and low $x$ have to be estimated. The contribution from $x>0.8$ is consistent with zero $(0.001 \pm 0.001)$. For the region $x<0.004$ a Regge like parametrization, $a x^{b}$, was fit to the data in the region $0.004<x<0.15$ and extrapolated to give a contribution to $S_{G}$ of $0.013 \pm 0.005$. The final result for $S_{G}$ is $0.235 \pm 0.026$, where the error is the sum in quadrature of the statistical and systematic contributions. The asymmetry $\bar{u}-\bar{d}$ is not something that can be generated by pQCD evolution and must be inserted by hand in the parametrizations of the parton distribution functions at the starting scale $Q_{0}^{2}$. We discuss this further in Sec. 6.1.

\section{Determining parton distribution functions and $\alpha_{s}$}

The success of pQCD, as worked out in the conventional framework outlined in Sec. 3, has been well documented in the textbooks of Roberts 6 and, more recently, Ellis, Stirling and Webber 141 . In the present section we consider how the conventional framework has been used to determine parton distributions and the 
strong coupling constant $\alpha_{s}$ (it is now conventional to quote the value of $\alpha_{s}$ at the scale $M_{Z}^{2}$, and this is what we shall mean by the notation $\alpha_{s}$ when we quote numerical values, unless otherwise specified). We discuss the extraction of parton distributions in Sec. 6.1. We pay particular attention to the extraction of the gluon distribution since most cross-sections at hadron colliders, present and future, are dominated by gluon induced processes, but the gluon distribution is the least accurately determined of the parton distributions. We summarize determinations of $\alpha_{s}$ from deep inelastic scattering, and compare with evaluations from other processes in Sec. 6.2. Finally we consider the non-perturbative techniques which have been used to predict the form of parton distributions in Sec. 6.3.

\subsection{Extraction of parton distributions}

Ideally one would like to find analytic parametrizations of parton distributions, or structure functions, which are consistent with pQCD. The problem is to perform a Mellin inversion of the exact predictions of pQCD for the moments of structure functions in order to find suitable analytic expressions for the structure functions themselves. Such parametrizations have been given by many authors, see for example 142,143 144.145. However, if one requires consistency with pQCD beyond leading order, one cannot find exact analytic expressions which are valid for more than a limited $x, Q^{2}$ range. For this reason, the most commonly used method of extracting parton distributions is to perform a direct numerical integration of the DGLAP equations at NLO. The technique is broadly as follows. An analytic shape for the parton distributions (valence, sea and gluon) is assumed to be valid at some starting value of $Q^{2}=Q_{0}^{2}$. This starting point is arbitrary, but should be large enough to ensure that $\alpha_{s}\left(Q_{0}^{2}\right)$ is small enough for perturbative calculations to be applicable. Then the DGLAP equations are used to evolve the parton distributions up to a different $Q^{2}$ value, where they are convoluted with coefficient functions, appropriate to the chosen renormalization scheme, in order to make predictions for the structure functions. These predictions are then fitted to the data, for whatever $x, Q^{2}$ points have been measured. The fit parameters are those necessary to specify the input analytic shape, and the QCD $\Lambda$ parameter (though one should note that this is fixed in some parametrizations). The input analytic form assumed for the parton distributions is only valid at the starting scale $Q_{0}^{2}$. For other $Q^{2}$ values the distributions must be interpolated from values at gridpoints, such as those provided in the parton distribution function library PDFLIB 146 (see also HEPDATA).

The DGLAP equations embody the predictions of pQCD in the NLLA at leading twist only. Thus one must be sure that the data which are fitted are not likely to be subject to strong higher twist corrections. An analysis of the need for higher twist terms was made by Virchaux and Milsztajn 147 on SLAC and BCDMS hydrogen and deuterium charged leptoproduction data. They accounted for target mass effects and included dynamical higher twist terms by allowing the leading twist form of the structure function to be multiplied by $\left(1+\frac{C}{Q^{2}}\right)$, where the parameter $C$ is fitted separately in each $x$ bin, so that no specific function of $x$ is imposed on the 
data. The values for $C$ are found to be non-zero and positive only for $x>0.5$ and $Q^{2} \lesssim 10 \mathrm{GeV}^{2}$, see Fig. 40 .

An analysis of data on the ratio $F_{2}^{\mu n} / F_{2}^{\mu p}$ by the NMC Collaboration 148 concluded that small higher twist terms were necessary to describe data in the kinematic region, $0.1<x<0.3,1<Q^{2}<10 \mathrm{GeV}^{2}$, but a more recent analysis of the latest NMC ratio data and comparison with SLAC and BCDMS data 138 , indicates that this ratio has only a small logarithmic $Q^{2}$ dependence compatible with leading twist pQCD predictions.

Thus higher twist contributions seem to be important only for high $x$ and low $Q^{2}$. It is interesting to note that the form of these contributions as measured experimentally is in agreement with the recent renormalon predictions 31 of the $1 / Q^{2}$ contribution to structure functions. These predictions differ for $F_{2}$ and $x F_{3}$, the predictions for $x F_{3}$ being small and negative for $x<0.65$ (see Fig. 40). This may explain longstanding differences in higher twist terms between charged lepton and neutrino data, whereby the analysis of EMC/SLAC proton target data 149 found large positive values of $C$ for $x>0.5$, whereas analysis of WA59 (anti)neutrino data 150 , found small negative values for most of the measured $x$ range. Unfortunately, the WA59 data did not distinguish $F_{2}$ and $x F_{3}$ for the higher twist analysis, and these data were taken on a heavy target without nuclear corrections. Thus these observations were not conclusive. However some analyses of the much more precise $\operatorname{CCER}(93)$ and $\operatorname{CCFR}(97) x F_{3}$ data 151.152 and of a combination of world $x F_{3}$ data 153, taking into account nuclear effects, has recently been made. The higher twist contribution is evaluated by adding a term $h(x) / Q^{2}$ to the to the pQCD prediction for $x F_{3}$ at leading twist, for each $x, Q^{2}$ point. Agreement of the measurements of $h(x)$ with the form of the renormalon prediction is again found.

In conclusion, the present consensus is that one may cut out the need for higher twist contributions by cutting out the kinematic region in which they are important. The cut has typically been made such that only data for which $Q^{2} \gtrsim 4 \mathrm{GeV}^{2}$, $W^{2} \gtrsim 10 \mathrm{GeV}^{2}$ are considered and most analyses have been made using the structure function $F_{2}$. Thus we note that: i) $F_{L}$ may be subject to higher twist corrections for all $x$, at low $Q^{2}$ (see Sec. 3.4); ii) the renormalon predictions indicate that $x F_{3}$ may have small higher twist corrections for all $x$; ii) if the $Q^{2}$ cut is lowered to $1 \mathrm{GeV}^{2}$, as in the most recent parametrizations, one should be more cautious (however since most of the data at such low $Q^{2}$ are also at low $x$, higher twist terms are not expected to be very significant). Subject to these warnings we may have confidence in leading twist DGLAP analyses. Of course the above considerations refer to the conventional higher twist effects discussed in Sec. 3.4. In HERA very low $x$ data we may see the effects of unconventional higher twist effects which contribute to parton shadowing as discussed in Sec. 7 .

When considering the applicability of QCD analyses of DIS data we should also consider the presence of diffractive-like events in the deep inelastic scattering event sample. It has been established that there is a contribution to this sample of approximately $10 \%$ from events with a large rapidity gap towards the proton remnant, 


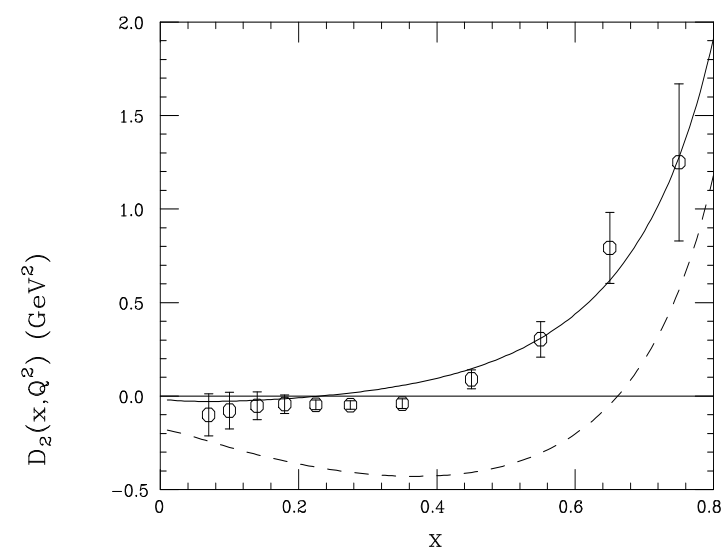

Fig. 40. Renormalon predictions for the twist- 4 contributions to $F_{2}$ (full line) and $x F_{3}$ (dotted line). The SLAC and BCDMS data from the analysis of Virchaux and Milsztajn are shown for comparison.

with characteristics as measured in hadronic diffractive exchange 3. . These events may originate from a different production mechanism as compared to the bulk of the deep inelastic scattering data, for example, they include processes such as exclusive vector meson production. So far no special account has been taken of this in the extraction of partons from inclusive $F_{2}$ measurements.

The most reliable parton distributions are obtained by making global analyses of a wide range of data from DIS and other hard processes. It is appropriate to first consider the consistency of data sets.

\subsubsection{Treatment of data sets in global analyses}

In addition to the standard corrections described in Sec. 5, there are further corrections which must be made when combining data from different experiments.

Firstly, data must be taken on comparable targets. A nuclear target cannot simply be treated as an additive combination of nucleons, and data taken on such a target have to undergo corrections for nuclear shadowing, binding and Fermi motion effects before they can be used to extract information on parton distributions within the free nucleon. Modern data are taken on hydrogen or deuterium targets to avoid this problem. However, for precision measurements, the need for correction even extends to deuterium targets. For (anti)neutrino beams the event rate on proton/deuterium targets is very low. Accordingly the CCFR experiment uses a heavy target and the data are corrected for nuclear effects before they are compared to the electroproduction data. Nuclear effects were measured extensively by the EMC, SLAC and NMC collaborations in the mid to late eighties 154 , so that reliable 
corrections are available for charged leptoproduction experiments.

Secondly, the treatment of heavy quark thresholds, needs careful consideration. The theoretical background to this has been given in Sec. 3.6. Different authors have used somewhat differing procedures, which will be indicated appropriately when their analyses are described. One should also be careful when comparing data from neutrino and muon/electron scattering, since the effect of mass thresholds will be different. In neutrino scattering charmed quarks can be produced directly from scattering off the strange sea, as well as in the boson-gluon fusion process (flavour excitation, $W^{*} s \rightarrow c$, as well as flavour creation, $W^{*} g \rightarrow s \bar{c}$ ) and the former process is dominant at the energies at which present day neutrino data have been taken. The neutrino data are usually corrected with a 'slow rescaling' prescription to allow for the charm mass threshold in the flavour excitation process 155 . However this is not really adequate when doing a NLO analysis and a full variable flavour treatment which also includes the flavour creation process should be performed 46 .

Thirdly, in the late 1980's there were still significant disagreements between various data sets. The muo-production experiments EMC 149 and BCDMS 91 disagreed in poth normalization and shape, so much so that the 1990 global analysis of HMRS 156, published two separate sets of parton distributions the E set for EMC data and the B set for BCDMS data. Similarly the neutrino production data of the CDHSW and CCFR collaborations differed in shape at low $x$. A complete re-analysis of the old SLAC experiment was undertaken incorporating modern understanding of relevant corrections 90 , partly in order to help to resolve these discrepancies. The EMC data have now been superseded by the increased precision of the NMC data, and the CDHSW data have been superseded by the increased precision of the CCFR data. There is a remaining disagreement between the NMC(97) Muon data and the CCFR(97) neutrino data at low $x$, see Fig. 33 and the discussion in Sec. 5.2. Modern global fits deal with this discrepancy differently, as specified in the next subsection. Differences in normalization between data sets are accounted for by allowing the relative normalizations of data sets to float within the overall absolute normalization uncertainties given by the experiments.

A further problem in the combination of data from different experiments concerns the treatment of systematic errors. Conventionally point to point systematic errors are accounted for by adding them in quadrature to the statistical errors. Most global fits do not take full account of correlations between experimental systematic errors since this information has not been available for all data sets and the correct procedure when combining different data sets is still a subject of active discussion.

\subsubsection{Global fits: the method of MRS and CTEQ}

During the 1980's and early 1990's many sets of parton distributions were developed to try to describe the available data $156.157,158.159$. Today only the parton distributions of the MRS 35. 60.36.161.37, CTEQ 162, 263.164 and GRV 38.39 groups are used, since only these take account of modern data. We first discuss the analyses of

MRS and CTEQ, which have a common philosophy. We begin by giving a general 
description of the parametrizations, discussing the assumptions which are made and the choice of data input to the analyses. This will be done in detail only for the MRS parametrizations, and significant differences for CTEQ will be pointed out when relevant.

The parton distributions must be parametrized by a suitably flexible analytic form at the starting $Q^{2}=Q_{0}^{2}$. MRS have used $Q_{0}^{2}=4 \mathrm{GeV}^{2}$, for all but the latest MRSR fits, whereas CTEQ have used $Q_{0}^{2}=2.56 \mathrm{GeV}^{2}$ for all but the CTEQ4LQ parametrization. Both groups use inputs which extend the simple forms of Eqs. 53, 54 as follows

$$
\begin{gathered}
x u_{v}=A_{u} x^{\eta_{1}}(1-x)^{\eta_{2}} P(x, u) \\
x d_{v}=A_{d} x^{\eta_{3}}(1-x)^{\eta_{4}} P(x, d) \\
x S=A_{S} x^{-\lambda_{S}}(1-x)^{\eta_{S}} P(x, S) \\
x g=A_{g} x^{-\lambda_{g}}(1-x)^{\eta_{g}} P(x, g)
\end{gathered}
$$

where $P(x, i)=\left(1+\epsilon_{i} \sqrt{x}+\gamma_{i} x\right)$ for MRS and $P(x, i)=\left(1+\gamma_{i} x^{\epsilon_{i}}\right)$ for the CTEQ analyses (although both groups have used 'minimal' parametrizations $P(x, i)=$ $\left(1+\gamma_{i} x\right)$, for the gluon distributions in some of their parametrizations). Not all of the normalizations $A_{i}$ are free parameters, $A_{u}, A_{d}$ are determined by the need to satisfy flavour sum rules and $A_{g}$ is determined in terms of the other three by the momentum sum rule. The distributions are usually defined within the $\overline{\mathrm{MS}}$ renormalization and factorization scheme and this will be assumed unless otherwise stated. The QCD scale parameter $\Lambda$ is usually a parameter of the fit. The treatment of $\Lambda$ across flavour thresholds is as given by Marciano 33 as described in Sec. 3.6. Since MRS express their results in terms of $\Lambda$ for four flavours, whereas CTEQ express their results in terms of $\Lambda$ for five flavours, we chose to quote $\alpha_{s}\left(M_{Z}^{2}\right)$ for both analyses.

Some comments on this choice of parametrization are in order. The fact that the $u_{v}$ valence shape is not the same as the $d_{v}$ valence shape has been known since the earliest days of neutrino scattering when ne and antineutrino scattering data on protons and deuterium were compared 165,166. However, in more recent years, the data which fix these she shapes have come from taking the difference and the ratios of $F_{2}^{\mu p}, F_{2}^{\mu n}$ data 138 . At large $x$, when only valence distributions are significant, one has

$$
\frac{F_{2}^{\mu n}(x)}{F_{2}^{\mu p}(x)}=\frac{1+4 d_{v}(x) / u_{v}(x)}{4+d_{v}(x) / u_{v}(x)}
$$

from the QPM. The data indicate that $d_{v}(x) / u_{v}(x) \sim(1-x)^{1} \rightarrow 0$ as $x \rightarrow 1$, as predicted by Field and Feynman 167, rather than the naive result of SU(3) flavour symmetry, $d_{v}(x) / u_{v}(x)=1 / 2$, for all $x$. (However, see references 166.169 for an alternative view.)

The sea distribution refers to $u, \bar{u}, d, \bar{d}, s, \bar{s}, c, \bar{c}$. Since we must have $q=\bar{q}$ within each flavour of the sea by definition, we may express the sea as $S=2(\bar{u}+\bar{d}+\bar{s}+\bar{c})$. Early parametrizations assumed that the $u, d$ content of the sea is flavour symmetric, but there is no mecessity for this and in 1992 NMC gave the first evidence that this is not the case 170. The Gottfried sum rule is violated (see Sec. 5.6 for the latest 
data). If we abandon the assumption that $\bar{u}=\bar{d}$, which was used in its derivation, we obtain

$$
\int_{0}^{1} \frac{d x}{x}\left(F_{2}^{p}-F_{2}^{n}\right)=\frac{1}{3} \int_{0}^{1} d x\left(u_{v}-d_{v}\right)+\frac{2}{3} \int_{0}^{1} d x(\bar{u}-\bar{d})
$$

Thus the observation of the vahne $\simeq 0.23$ for this sum tells us that $\bar{d}>\bar{u}$, as expected from Paliompression 6 . (For further discussion of the reasons for this result see refs. 171 172, 173). In the MRS parametrization this is taken into account by expressing the structure of the sea as

$$
2 \bar{u}=0.4(1-\delta) S-\Delta, 2 \bar{d}=0.4(1-\delta) S+\Delta, \quad 2 \bar{s}=0.2(1-\delta) S, 2 \bar{c}=\delta S
$$

where,

$$
x \Delta=x(\bar{d}-\bar{u})=A_{\Delta} x^{\lambda_{\Delta}}(1-x)^{\eta_{S}} P(x, \Delta)
$$

with $\epsilon_{\Delta}=0$, and $\lambda_{\Delta} \sim 0.5$ from considering the breaking of $\rho / A_{2}$ Regge trajectory exchange degeneracy. The CTEQ group account for $u, d$ differences in the sea similarly, but allow more free parameters.

The strange sea is suppressed relative to the $u$ and $d$ seas and the charmed sea is very suppressed. The origin of this suppression lies in their larger quark masses, but we may allow for it by introducing a simple suppression factor. MRS assume that $\bar{s}=(\bar{u}+\bar{d}) / 4$, i.e. the strange sea is suppressed by $50 \%$ compared to the $u$ and $d$ sea distributions at $Q_{0}^{2}$. The justification for this comes from CCFR opposite sign dimuon data 108, which confirm the earlier data of CDHSW 174. Briefly, opposite sign dimuon events dominantly arise in (anti)neutrino scattering when the struck quark is an $(\bar{s}) s$ quark which becomes a $(\bar{c}) c$ quark through the flavour changing weak current. This $(\bar{c}) c$ quark then decays through the muon channel to $\left(\bar{s} \mu^{-} \nu_{\mu}\right)$ $s \mu^{+} \nu_{\mu}$ yielding a muon of opposite sign to that at the original lepton scattering vertex. The rate for the process thus gives a measure of the size of the strange sea, which is consistent with $50 \%$ suppression $^{\circ}$. The strange sea is treated similarly in the latest CTEQ and MRS parametrizations, although historically CTEQ had allowed it to have an independent parametrization. Their early work on extracting the size of strange sea from the difference $x s(x)=\frac{5}{6} F_{2}^{\nu N}(x)-3 F_{2}^{\mu d}(x)$, which involves combining data from two different experiments, pointed to a discrepancy between this determination and the dimuon results, indicating a difference between NMC muon data and CCFR neutrino data, in the region $0.01<x<0.1$. As we have seen this discrepancy has still not been resolved even when the updated CCFR(97) and NMC(97) data are used (see Fig. 33). CTEQ omit these data points from their subsequent analyses, whereas MRS include both sets of data to achieve a compromise fit.

The charmed sea has also been treated similarly by the two groups. Their standard parametrizations use a zero-mass variable flavour number (ZM-VFN) scheme, see Sec. 3.6. Basically one assumes that $c\left(x, Q^{2}\right)=0$ for $Q^{2} \leq m_{c}^{2}$. The charm content of the nucleon at higher $Q^{2}$ is then generated by the boson-gluon fusion process, as embodied in the DGLAP equations for massless partons. The shape

\footnotetext{
o The description of the dimuon data is better if this suppression is applied at $Q_{0}^{2}=1 \mathrm{GeV}^{2}$, rather than $Q_{0}^{2}=4 \mathrm{GeV}^{2}$.
} 
of the charm distribution resulting at $Q_{0}^{2}=4 \mathrm{GeV}^{2}$ is similar to the shape of the overall sea distribution. The magnitude of the charm contribution to the total sea distribution depends sensitively on $m_{c}$. Data on $F_{2}^{c \bar{c}}$ from the EMC collaboration have been used to fix $m_{c}^{2}=2.7 \mathrm{GeV}^{2}$, and this results in $2 \%$ of the total sea content being charmed at $Q_{0}^{2}=4 \mathrm{GeV}^{2}$ p. The contributions of bottom and top quarks are treated similarly, and they turn out to be negligible for present analyses. Clearly such a treatment of heavy quarks can only be valid far above the relevant thresholds, $W^{2} \gg 4 m_{q}^{2}$. Near threshold a proper treatment of quark mass effects is required as discussed in Sec. 3.6. New parton distributions which incorporate a consistent treatment of heavy quark effects from the threshold region to the asymptotic regime, using general-mass variable flavour number (GM-VFN) schemes, have been given by Martin et al 15 and Lai and Tung 44 . These will be discussed further in Sec. 6.1.4.

The form $x^{-\lambda_{g}}$ for the gluon parametrization at small $x$ is suggested by Regge behaviour as explained in Sec. 3, but whereas the conventional Regge exchange is that of the soft Pomeron, with $\lambda_{g} \sim 0.0$, one may also allow for a hard Pomeron, with $\lambda_{g} \sim 0.5$. The reasons for this are discussed in detail in Sec. \&. In the original MRSD 35 parametrizations these values were fixed as the alternative possibilities $\mathrm{MRSD}_{0}$ and $\mathrm{MRSD}_{-}$. However the input of high precision HERA data to the fits has allowed $\lambda_{g}$ to be left as a free parameter.

The form $x^{-\lambda_{S}}$ in the sea quark parametrization comes from similar considerations since, at small $x$, the process $g \rightarrow q \bar{q}$ dominates the evolution of the sea quarks. Hence the fits to early HERA data have as a constraint $\lambda_{S}=\lambda_{g}$. However one only expects this once $Q^{2}$ is large enough for the effect of DGLAP evolution to be seen, hence it may not be a reasonable constraint at $Q^{2}=Q_{0}^{2}$. Furthermore, the exact solution of the DGLAP equations predicts $\lambda_{S}=\lambda_{g}-\epsilon$, see Eq. 164. The data at low $x$ are now of sufficient precision to allow $\lambda_{g}$ and $\lambda_{S}$ to be separate free parameters, as in the MRSR 37 and CTEQ4 164 fits.

The relationship between the measured structure functions and the parton distributions is not straightforward at NLO since the evolved parton distributions must be convoluted with coefficient functions and all types of parton may contribute to a particular structure function through the evolution. However, the simple LO formulae give a good guide to the major contributions. At LO the relationships between the structure functions and parton distributions (ignoring charm) may be written as

$$
\begin{gathered}
F_{2}^{\mu p}-F_{2}^{\mu n}=\frac{1}{3} x(u+\bar{u}-d-\bar{d}) \\
\frac{1}{2}\left(F_{2}^{\mu p}+F_{2}^{\mu n}\right)=\frac{5}{18} x\left(u+\bar{u}+d+\bar{d}+\frac{4}{5} s\right) \\
F_{2}^{\nu N}=F_{2}^{\bar{\nu} N}=x(u+\bar{u}+d+\bar{d}+2 s) \\
\frac{1}{2} x\left(F_{3}^{\nu N}+F_{3}^{\bar{\nu} N}\right)=x(u-\bar{u}+d-\bar{d})
\end{gathered}
$$

p If one is using $Q_{0}^{2}=1 \mathrm{GeV}^{2}$, then $m_{c}^{2}=2.7 \mathrm{GeV}^{2}$ is within the fitted region and one must deal with the threshold behaviour smoothly. The formalism Georgi and Politzer has been used to suppress the generated charm density by smooth factor 175 . 
Thus, even if there were no discrepancies between different data sets, deep inelastic data could only determine four quantities (usually taken to be $u+\bar{u}, d+\bar{d}, \bar{u}+\bar{d}$ and $s$ ) with any precision, on a point by point basis. Thus the global fits use information from non-DIS processes in order to have more complete information on parton distributions.

We will first consider data which constrain quark distributions. Drell Yan dilepton production in the process $p N \rightarrow \mu^{+} \mu^{-} X$ can be a sensitive probe of the sea quark distribution since the dominant subprocess is $q \bar{q} \rightarrow \gamma^{*}$, and thus the crosssections are directly proportional to the antiquark distributions. The E605 data 176 have been used to constrain the sea distribution at medium to large $x$ values, $0.1<x<0.6$, yielding $\eta_{S} \sim 10$. More recently, the CDF data 177 provide a further constraint at $x \sim 0.01$.

The NA51 data 178 on the asymmetry, $A_{D Y}$, between the differential crosssections $\frac{d^{2} \sigma}{d M d y}$ at $y=0$ for the processes $p p \rightarrow \mu^{+} \mu^{-} X$ and $p n \rightarrow \mu^{+} \mu^{-} X$ (where $M$ and $y$ are the invariant mass and rapidity of the lepton pair) has given more information on the difference $\bar{d}-\bar{u}$. The dominant subprocesses are $u \bar{u}, d \bar{d} \rightarrow \gamma^{*}$, and the partons are to be evaluated at $x=M / \sqrt{s}$. Hence the NA51 measurement serves to fix $(\bar{d}-\bar{u})$ at the $x$ value, $x \sim 0.18$. The E866 experiment is now taking data which will give further information on the $\bar{u} / \bar{d}$ asymmetry in the $x$ range $0.05<x<0.3179$.

Data on $W$ production may also be used to investigate quark distributions. The processes $p \bar{p} \rightarrow W^{+}\left(W^{-}\right) X$ proceed via the subprocesses $u \bar{d} \rightarrow W^{+}(d \bar{u} \rightarrow$ $W^{-}$), and the cross-sections are thus sensitive to the $u$ and the $d$ distributions at $x \sim M_{W} / \sqrt{s}$, i.e. $x \sim 0.13$ at $\mathrm{CERN}$, where UA2 data are taken, and $x \sim 0.05$ at FNAL, where CDF 180 and D0 data are taken. The $W^{ \pm}$charge asymmetry, $A_{W}(y)$, probes the slope of the $d / u$ ratio, since the $u$ quarks carry more momentum on average than the $d$ quarks and so the $W^{+}$tend to follow the direction of the incoming proton and the $W^{-}$that of the antiproton. These measurements give a more direct probe of the $d / u$ distribution than the $F_{2}^{n} / F_{2}^{p}$ ratio at intermediate $x$ values, since $W$ production, at Tevatron energies, is less sensitive to the contribution of the antiquarks.

The need for additional information from non-DIS processes is more pronounced for the gluon distribution. Since the gluon does not couple to the photon it does not enter the expressions for the structure functions at all at LO. It is merely constrained by the momentum sum rule, and by the way the DGLAP equations feed its evolution into the sea distribution ${ }^{\mathrm{q}}$. Consequently the gluon is the parton which has the largest uncertainty. We devote Sec. 6.1.3 to considering the gluon distribution. We do not confine our discussion to considering the role of the gluon distribution in the global fits. We consider ways to extract the gluon distribution from DIS using the scaling violations of $F_{2}$, paying particular attention to the fits done by the individual experiments and we consider information which can

q At NLO the gluon distribution may contribute to $F_{2}$ depending on the renormalization scheme chosen. 
be gained from the measurement of other structure functions and from non-DIS processes, both at present and in future.

\subsubsection{Methods of extracting the gluon distribution}

An overview of the data than can contribute to the gluon extraction is given in Fig. 41 as function of $x$.

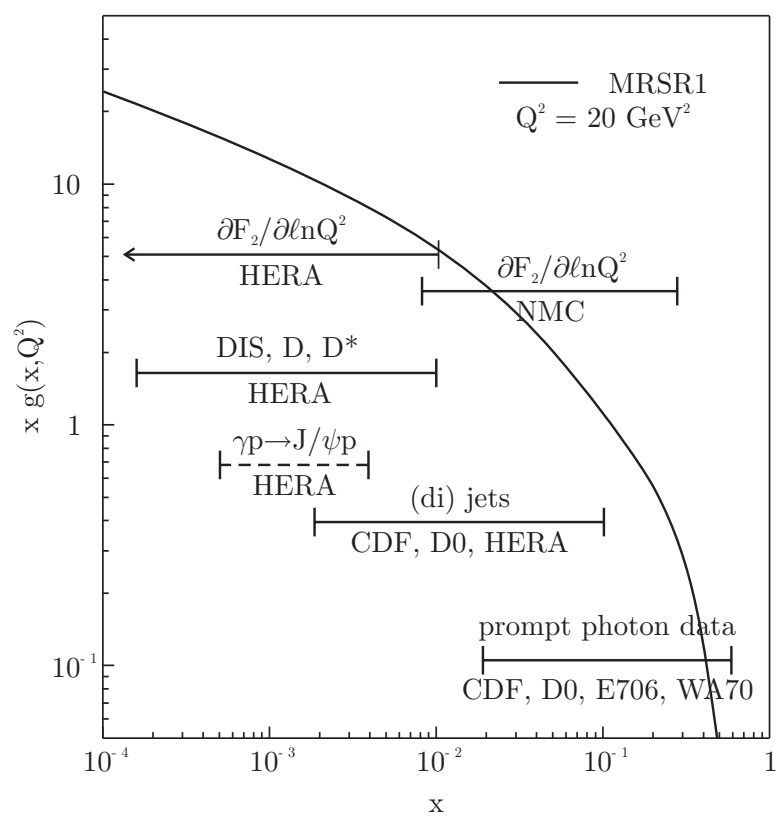

Fig. 41. The $x$ intervals in which the gluon may be constrained by various sets of data. The MRSR1 gluon distribution is superimposed.

We begin by considering the QCD fits made by individual experiments to their own structure function data. The experimental collaborations have the advantage of knowing the correlations between their systematic errors and thus can produce reliable error bands on the extracted distributions. The usual method follows that of the global fits. A functional form with few free parameters is assumed for the gluon at a chosen starting scale, and the coupled singlet evolution equations (Eqs. 61, 62) are solved to NLO simultaneously (usually in the $\overline{\mathrm{MS}}$ scheme). Since the gluon distribution is strongly correlated with the value of $\alpha_{s}$ (see Sec. 6.2), a fixed value of $\alpha_{s}$, as determined from independent data, is often assumed. Higher twist contributions must be included in the formalism if high $x$, low $Q^{2}$ data are included.

In 1992 Virchaux and Milsztajn 147 made a fit to combined SLAC and BCDMS proton and deuteron data, which accounted for higher twist terms and for the correlation with $\alpha_{s}$. The CDHSW collaboration 117 made a similar fit to the singlet 
structure function $F_{2}$ extracted from neutrino scattering on iron. These extractions indicated a soft behaviour of the gluon at high $x$ and a tendency for $x g(x)$ to rise for $x \leq 0.1$. However, the errors on the gluon distribution were large, approximately $30 \%-50 \%$ at $x \sim 0.1$.

In 1993 the NMC collaboration used their precise $F_{2}$ data on proton and deuteron targets in the kinematic range $0.008 \leq x \leq 0.5,1 \leq Q^{2} \leq 48 \mathrm{GeV}^{2}$ to make an NLO QCD analysis 181 . The flavour singlet and non-singlet quark distributions as well as the gluon distribution were parametrized at the reference scale $Q_{0}^{2}=7$ $\mathrm{GeV}^{2}$ and all data with $Q^{2} \geq 1 \mathrm{GeV}^{2}$ were included in the fit. Higher twist terms were included by using the forms extracted by Virchaux and Milsztajn (see Fig. 40) averaged over the proton and deuteron and suitably extrapolated to lower values of $x$. Their contribution was found to be substantial at high $x$ for scales of about $1 \mathrm{GeV}^{2}$. The gluon distribution extracted is shown in Fig. 44 for the fixed value of $\alpha_{s}=0.113$. The uncertainty on the distribution is calculated taking into account the statistical errors, the systematic errors including correlations, the contribution of the unmeasured region, and the uncertainties on $\alpha_{s}$ and higher twist contributions. The total uncertainty at $x=10^{-2}$ amounts to $\sim 20 \%$. These data cover the region $x>0.01$ and the gluon distribution shows a tendency to continue to rise moderately at low $x$. HERA data have now extended our knowledge of the gluon distribution down to $x \sim 10^{-4}$.

Fig. 29 shows clearly the scaling violations for the measured low $x$ values at HERA. Before full NLO DGLAP QCD fits were applied to extract the gluon from the HERA data, several approximate methods were used to deconvolute the gluon density directly from $F_{2}$, exploiting the fact that, at low $x$ the gluon is by far the most dominant parton and $F_{2}$ is essentially given by the singlet sea quark distribution which is driven by the gluon (through the $g \rightarrow q \bar{q}$ splitting process). These methods gave a first impression of the behaviour of the gluon density at low $x$. The simplest method proposed by J. Prytz 182 consists of neglecting the quark contribution to scaling violations and performing a Taylor expansion of the splitting function around $x=\frac{1}{2}$, leading to a very simple LO expression for the gluon density

$$
x g\left(x, Q^{2}\right) \approx \frac{27 \pi}{20 \alpha_{s}\left(Q^{2}\right)} \frac{\partial F_{2}\left(\frac{x}{2}, Q^{2}\right)}{\partial \ln Q^{2}}
$$

This is a crude approximation which is expected to hold only to within $20 \%$ at $x=$ $10^{-3}$ for a steeply rising gluon 183 . The results are shown in Fig. 42, using the H1(93) data. This demonstrated the strikingly strong rise of the gluon distribution at low $x$. Approximate NLO corrections to this approach have been calculated 184.182 and several more sophisticated and accurate methods have also been proposed 185, 186. These have usually involved the need for some assumption about the degree of singularity of the behaviour of $F_{2}$ or the gluon itself as $x \rightarrow 0$. Experimentalists have preferred to concentrate on gluon extractions from full NLO DGLAP fits to their data.

Before we describe these fits we draw attention to the assumptions inherent 
in such extractions. It is implicitly assumed that the evolution equations can be applied in the kinematic range studied. It is not guaranteed that this is the case for DGLAP evolution at low $x$, it may be necessary to consider the BFKL 187 , or other, evolution equations, as will be discussed in Sec. 7. We illustrate this by comparing the gluon distribution extracted from a LO DGLAP fit to that extracted from a fit using somewhat different evolution equations, namely a hybrid set 188 , based on the DGLAP and the BFKL evolution equations. (The latter was only available for the gluon, hence for the evolution of the quark distribution the DGLAP equations are used, suitably matched to the BFKL evolved gluon distribution). The results are shown in Fig. 42. Both fits are made to NMC(95), BCDMS and H1(93) proton data, where the fixed target data is introduced to constrain quark densities at high $x$. The error bands show the statistical and total errors (taking into account correlations between the systematic uncertainties) of the DGLAP fit, which agrees very well with the approximate method of Prytz. The full line shows the hybrid fit result. One can see that a different, in this case steeper, gluon distribution can emerge when using different evolution equations even with the same input data.

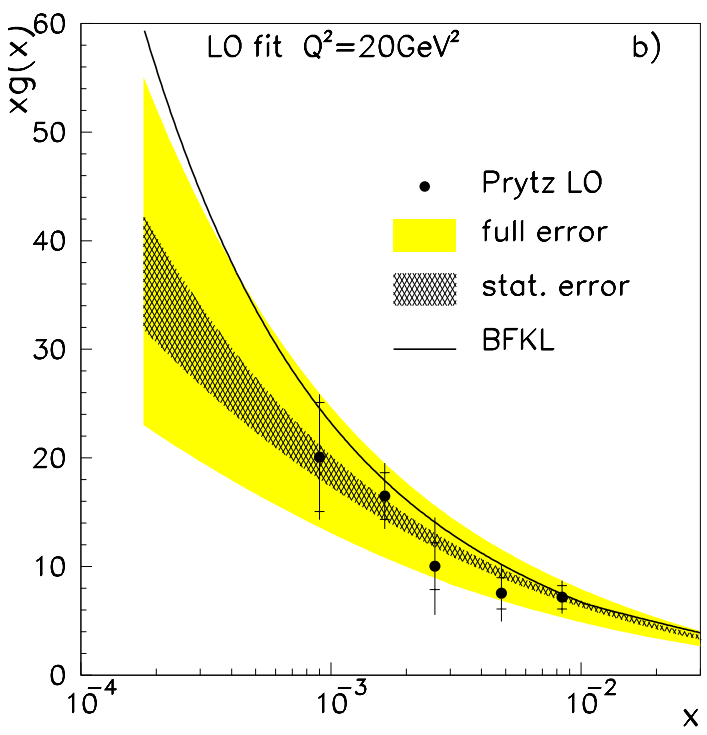

Fig. 42. The gluon density at $20 \mathrm{GeV}^{2}$ from a LO fit (shaded) and a mixed DGLAP-BFKL fit (full line). The points are calculated using the method of Prytz on H1(93) data. The inner error bars (band) represent the statistical errors, the outer error bars (band) the statistics and systematics added in quadrature.

Both the $\mathrm{H} 1$ and the ZEUS collaborations have made fits of their data to numerical solutions of the DGLAP equations at NLO in the $\overline{\mathrm{MS}}$ scheme. Heavy quark contributions have been treated using the three fixed flavour number scheme (FFN) given in ref. 40 , and extended to $\alpha_{s}^{2}$ in the coefficient functions according to ref. 2. 
Thus there is no charmed parton distribution, charm is dynamically generated by the boson-gluon fusion (BGF) process. The scale for this process has been taken as $\sqrt{Q^{2}+4 m_{c}^{2}}$, with a charm quark mass of $m_{c}=1.5 \mathrm{GeV}$. An uncertainty in the charm quark mass of $\sim 0.5 \mathrm{GeV}$ leads to a few percent variation of the gluon density. The small contribution of beauty quarks has been neglected.

Both groups use parametrizations for the input parton distributions which are very similar to those used by the global fits of MRS and CTEQ, see Eqs. 139142. For the $\mathrm{H} 1$ fit 189 the the input scale is taken to be $Q_{0}^{2}=5 \mathrm{GeV}^{2}$ and $\mathrm{H1}(94)$ data with $Q^{2}>5 \mathrm{GeV}^{2}$ are included in the fit. The function $P(x, i)$ is given by $P(x, i)=\left(1+\epsilon_{i} \sqrt{x}+\gamma_{i} x\right)$ for quark distributions and by $P(x, g)=1$ for the gluon distribution. Since no isoscalar data are available yet in the small $x$ domain, $\eta_{1}=\eta_{3}$ is assumed. The quark and antiquark components of the sea are assumed to be equal, and the flavour composition of the sea is assumed to be $25 \%$ strange and equal amounts of $u$ and $d$ flavour (such that the Gottfried sum rule would not be violated). The normalizations of the valence quark densities are fixed using the flavour sum rules and the normalization of the gluon density is determined using the momentum sum rule. The parameters $\lambda_{S}$ and $\lambda_{g}$ which describe the low $x$ behaviour of the sea and gluon distributions are free parameters which are allowed to be different from each other. The value of $\alpha_{s}$ is fixed at $\alpha_{s}=0.113$, as determined in ref.147.

The ZEUS fit 190 differs in the following respects. The input scale is taken to be $Q_{0}^{2}=7 \mathrm{GeV}^{2}$ but ZEUS(94) data for $Q^{2}>1.5 \mathrm{GeV}^{2}$ are included in the fit. The gluon distribution uses the function $P(x, g)=\left(1+\gamma_{g} x\right)$. Rather than parametrizing the $x u_{v}, x d_{v}$ and $x S$ distributions, ZEUS chose to parametrize the singlet quark distribution $x \Sigma$ and the non-singlet difference between up and down quarks in the proton $x \Delta_{u d}$ as follows

$$
\begin{aligned}
x \Sigma(x) & =A_{\Sigma} x^{\lambda_{\Sigma}}(1-x)^{\eta_{\Sigma}}\left(1+\epsilon_{\Sigma} \sqrt{x}+\gamma_{\Sigma}\right), \\
x \Delta_{u d}(x) & =A_{u d} x^{\eta_{u d 1}}(1-x)^{\eta_{u d 2}} .
\end{aligned}
$$

The sea quark density is obtained by subtracting the valence distribution (taken from the $\mathrm{MRSD}_{-}^{\prime}$ parametrization, which is still appropriate for the higher $x$ valence region) from the singlet distribution, and the strange sea is assumed to be $20 \%$ of the total sea.

In order to constrain the valence quark densities at high $x$, proton and deuteron results from the BCDMS (H1 fit only) and NMC (both H1 (NMC(95)) and ZEUS $(\mathrm{NMC}(97)) \mathrm{fit})$ experiments are also used. In the fitting procedure a term was added to the $\chi^{2}$ to permit variation of the relative normalizations of the different data sets. To avoid possible contributions from higher twist effects, NMC data for which $Q^{2}<4 \mathrm{GeV}^{2}$ were excluded from the ZEUS fit, and NMC and BCDMS data for which $Q^{2}<5 \mathrm{GeV}^{2}$, and $Q^{2}<15 \mathrm{GeV}^{2}$ if $x>0.5$, were excluded from the H1 fit. The $\chi^{2}$ obtained using uncorrelated systematic errors and the $\chi^{2}$ computed when considering the full error of each point are given in Table 1 for the H1 fit. 
Table 4. The $\chi^{2}$ values for the H1 NLO QCD fit. For each experiment the number of data points used in the QCD fit, the $\chi^{2}$ obtained as described in the text using only the uncorrelated errors, the $\chi^{2}$ computed from the same fit using the full error on each point and the normalization factors as determined from the fit, are given. The $\mathrm{H} 1$ nominal vertex and shifted vertex data samples are denoted as NVX and SVX respectively.

\begin{tabular}{cccccccc}
\hline Experiment & $\begin{array}{c}\text { H1(94) } \\
\text { NVX }\end{array}$ & $\begin{array}{c}\text { H1(94) } \\
\text { SVX }\end{array}$ & NMC(95)-p & NMC(95)-D & BCDMS-p & BCDMS-D & total \\
\hline data points & 157 & 16 & 96 & 96 & 174 & 159 & 698 \\
$\chi^{2}$ (unco. err.) & 174 & 13 & 157 & 153 & 222 & 208 & 931 \\
$\chi^{2}$ (full error) & 85 & 6 & 120 & 114 & 122 & 140 & 591 \\
normalization & 1.00 & 1.04 & 1.00 & 1.00 & 0.97 & 0.97 & \\
\hline
\end{tabular}

Only small adjustments of the relative normalizations are required demonstrating remarkable agreement between these different experiments.

The results of the ZEUS fit have been shown in Fig. 27 and Fig. 28 versus $x$ and Fig. 29 versus $Q^{2}$. The fit gives a good description of all data used. The $Q^{2}$ dependence at fixed $x$ is also described well over the nearly four orders of magnitude covered by the HERA data, see Fig. 29. Adding the statistical and systematic errors in quadrature the quality of the ZEUS fit is $\chi^{2}=463$ for 408 data points and 13 free parameters.

For ZEUS the parameters describing the low $x$ behaviour of the quark singlet and gluon distributions at $Q_{0}^{2}=7 \mathrm{GeV}^{2}$ are $\lambda_{\Sigma}=0.23$ and $\lambda_{g}=0.24$. For the H1 fit the corresponding dependences of the sea and the gluon distributions at $Q_{0}^{2}=5 \mathrm{GeV}^{2}$ are $\lambda_{S}=0.19$ and $\lambda_{g}=0.20$. These powers are similar to those extracted from the global fits, see Sec. 6.1.4 and Fig. 46. Note that there are sizeable correlations between the fit parameters which were not studied in detail as the basic aim of these analyses was to extract the gluon density.

Fig. 43 shows the NLO gluon density $x g\left(x, Q^{2}\right)$ at $Q^{2}=5 \mathrm{GeV}^{2}$ and $Q^{2}=$ $20 \mathrm{GeV}^{2}$ as extracted from the $\mathrm{H} 1$ fit. Note that there are no $F_{2}$ measurements below $5 \cdot 10^{-4}$ at $Q^{2}=20 \mathrm{GeV}^{2}$, but in that region the gluon is constrained by the data at lower $Q^{2}$ via the QCD evolution equations. The error band results from the statistical and systematic errors added in quadrature, taking into account correlations. A variation of $\alpha_{s}$ by 0.005 would give a change of $9 \%$ in the gluon density at $20 \mathrm{GeV}^{2}$. The striking rise of the gluon density towards low $x$ is confirmed and this rise increases with increasing $Q^{2}$.

Fig. 44 shows $x g(x)$ at $Q^{2}=20 \mathrm{GeV}^{2}$ for both $\mathrm{H} 1$ and ZEUS. The error bands account for statistical and systematic errors including correlations, for both fits. The agreement is good, the gluon density extracted by ZEUS being somewhat lower than that extracted by H1. At the lowest $x$ values $\sim 10^{-4}$ the gluon distribution is now determined with a precision of about $20 \%$, an impressive improvement in reach and precision compared with the previous fits shown in Fig. 42. At around $x=0.01$ the HERA fits make contact with the fit performed by the NMC collaboration on their own data. The agreement with the NMC result is very good.

A fit following the $\mathrm{H} 1$ prescription (but with fixed $\alpha_{s}=0.118$ ) was made using 


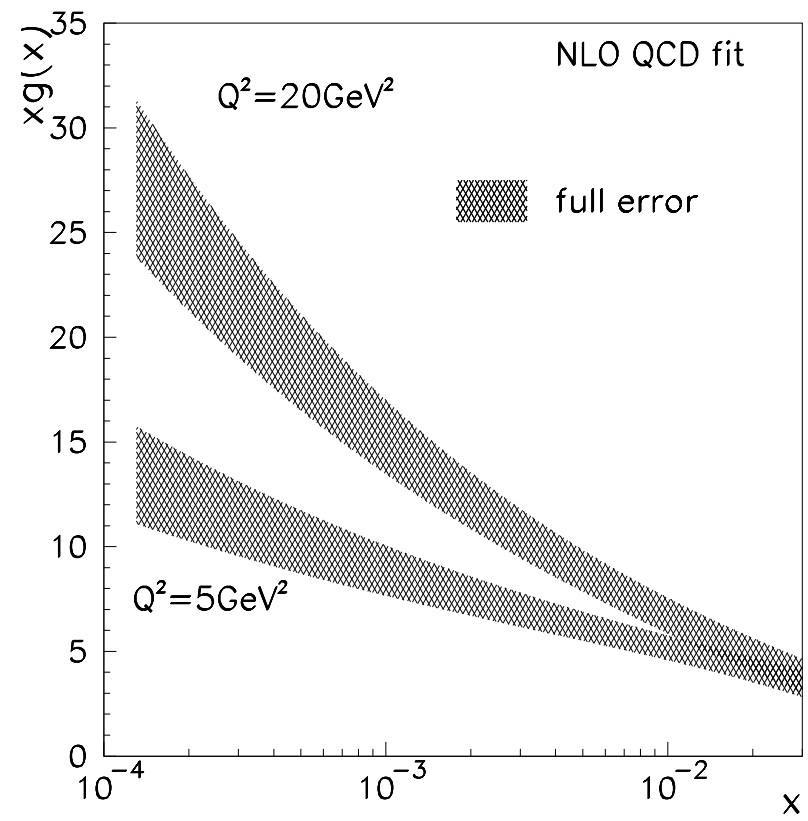

Fig. 43. The gluon density $x g(x)$ at $Q^{2}=5 \mathrm{GeV}^{2}$ and $Q^{2}=20 \mathrm{GeV}^{2}$ extracted from the H1 NLO DGLAP fit. The error bands represent statistical and systematic errors taking into account correlations.

both H1(94) and ZEUS(94) data, as well as NMC(97) and BCDMS fixed target data. Only HERA(94) data for which $Q^{2}>5 \mathrm{GeV}^{2}$ were used in this fit. The result (central value) is also shown in Fig. 44 and is close to the ZEUS fit result. Fig. 44 also shows a collection of recent determinations from the global analyses of CTEQ, GRV and MRS. Although these analyses include additional data to constrain quark and gluon distributions, the resulting gluon distributions are broadly similar to the HERA fits and to each other in the fitted region. However they are starting to deviate from each other at the lowest $x$ values.

It is interesting to investigate whether there is any evidence for higher twists in the low $x$ HERA data. ZEUS have included BCDMS and SLAC data in their fit in order to extend it to lower $Q^{2}$. Fits are made for various starting scales down to $Q_{0}^{2}=0.4 \mathrm{GeV}^{2}$ and all data above the starting scale are included in the fit. Higher twist terms of the form $f(x) / Q^{2}$ are included in the fit and the value of $f(x)$ is fitted in each $x$ bin. It is found that the higher twist contribution is negligible for $x<10^{-2}$, even for low $Q^{2}$ data. The fit is unable to describe the data if a starting scale $Q_{0}^{2}<0.8 \mathrm{GeV}^{2}$ is chosen, independent of whether or not such higher twist terms are included. This breakdown of the applicability of the DGLAP equations at low $Q^{2}$ is discussed further in Sec. 8 . 


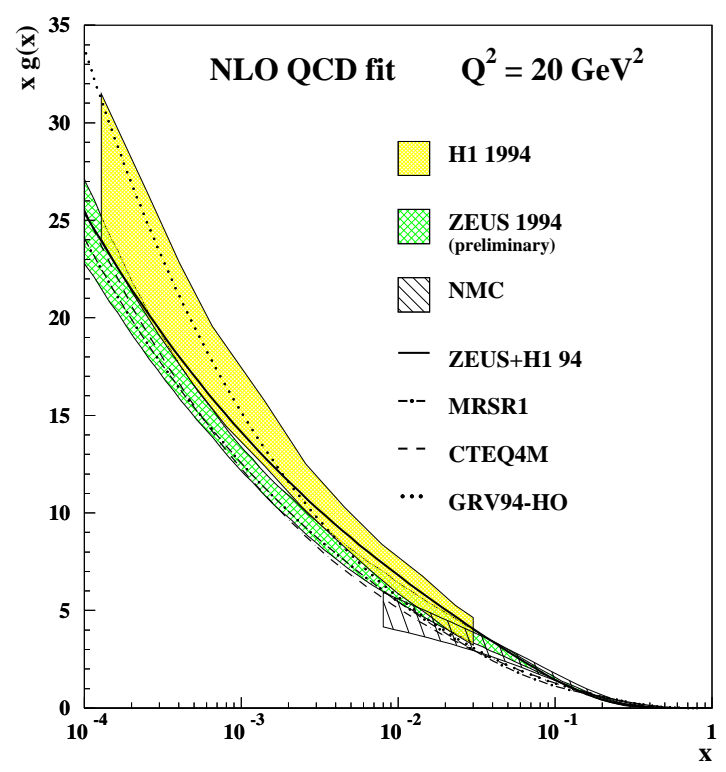

Fig. 44. The gluon density $x g(x)$ at $Q^{2}=20 \mathrm{GeV}^{2}$ extracted from a NLO DGLAP fits, for ZEUS and H1. The NMC fit is also shown at higher $x$. The solid line is from a new fit using all HERA(94) data. The predictions from CTEQ4 (dashed), GRV94 (dotted) and MRSR1 (dashed-dotted) are also shown for comparison

A common problem in extracting the gluon using only structure function data is the limited sensitivity to the gluon at large $x$. This is demonstrated in a fit of the HERA(94) and fixed target data using a Bayesian treatment of the systematic errors, by Alekhin 191. The resulting uncertainty on the gluon at $x>0.3$ is essentially $100 \%$. Thorne 125 has shown that using information from charm production in DIS and prompt photon production in non-DIS processes can reduce this uncertainty significantly, even when these data are only used to impose weak constraints. The global analyses of MRS, CTEQ and GRV have always included additional data from non-DIS processes in their fits in order to constrain the gluon. Data on prompt photon production and single inclusive jet data from hadronic collisions have been used to date.

Prompt photon data have been used for the $x$ range: $0.02<x<0.5$, see Fig.41. The process $p N \rightarrow \gamma X$ should provide information on the gluon distribution since the dominant subprocess is $g q \rightarrow \gamma q$, at leading order. However, one must also account for non-direct $\gamma$ production in fragmentation processes from other partons 192. There is also some uncertainty from factorization and renormalization scale dependence. The most reliable information comes from the lower energy WA70 data on $p p \rightarrow \gamma X 193$ which constrains the gluon in the $x$ range, $0.3 \leq x \leq 0.5$. 
There are also newer fixed target data from UA6 94 and E706 195 which cover the same $x$ range as WA70 and there are ISR data 196. which extend to lower $x$, $(0.15 \leq x \leq 0.3)$, and data from UA2 197 and CDF 198 which extend into the medium-small $x$ region $(0.05 \leq x \leq 0.15$ and $0.02 \leq x \leq 0.1$, respectively). These higher energy data are sufficiently accurate that one can see that the shape of the $E_{T}$ distribution of the photons with respect to the beam axis is steeper than that predicted by $\mathrm{pQCD}$ calculations (even after correction for fragmentation effects). This discrepancy can be remedied by including the effects of 'intrinsjc' $k_{T}$,i.e. $k_{T}$ broadening of initial state partons due to soft gluon radiation 163, 161, 199.200. Such broadening would have to increase with $\sqrt{s}$ in order to describe all the data 201. Until these effects are calculated in QCD it is difficult to use these higher energy data to pin down the gluon202 203204. For the lower energy data of WA70 and E706 the broadening is much less, but even here there are the scale uncertainties which lead to an uncertainty of $\sim 25 \%$ in the gluon distribution. The MRS and GRV fits use the WA70 data but the recent CTEQ analyses consider the uncertainty to be sufficiently serious that they either have not used these data or have introduced a scale parameter to account for this uncertainty.

Further information on the gluon may come from the single jet inclusive crosssections for jets with transverse energies $E_{T} \sim 100 \mathrm{GeV}$, since these are dependent on the gluon via $g g, g q$ and $g \bar{q}$ initiated subprocesses. There are high statistics jet measurements from the CDF and D0 collaborations at $\sqrt{s}=1.8 \mathrm{TeV} 205$.206. For jets with transverse energy $50 \lesssim E_{T} \lesssim 200 \mathrm{GeV}$ pQCD calculations are considered reliable. The slope of the $E_{T}$ distribution constrains the combination $\alpha_{s}\left(\mu^{2}\right) g\left(x, \mu^{2}\right)$ at the scale $\mu^{2}=E_{T}^{2}$ and $x=2 E_{T} / \sqrt{s} \sim 0.1$. Currently the jet data are more sensitive to $\alpha_{s}$ than to the gluon distribution. However an independent measure of $\alpha_{s}$, would allow us to use these data to constrain the gluon distribution. The CTEQ collaboration have included the jet data in their CTEQ4 fits whereas MRS merely compare their results to the jet data. Finally we note that the CDF jet data for $E_{T}>200 \mathrm{GeV}$ rise above the predictions from most conventional global fits such as MRSR and CTEQ4. This has given rise to many interesting suggestions of new physics effects 207. However CTEQ have found a conventional parametrization (CTEQ4HJ) 208 which is able to describe these data. Another conventional explanation is given by Klasen and Kramer 209.

We now briefly review other processes which may yield information on the gluon in future. Dijet production in high energy $p \bar{p}$ collisions can give information on the small $x$ gluon. If the jets are produced with equal transverse momentum and very forward (with pseudorapidity $\eta>>1$ ) then the gluon distribution can be probed down to about $x=0.005$, for $\sqrt{s}=1.8 \mathrm{TeV} 210$.

Dijet production initiated by the processes $\gamma g \rightarrow q \bar{q}$ and $\gamma q \rightarrow q g$, in DIS at HERA, have also been used to extract the gluon distribution. A LO extraction has been presented by H1211. The NLO corrections are known and the effects of scheme dependence and jet algorithms have been quantified 212. A method to extract the gluon density in NLO via an analysis in Mellin space has been proposed 
in ref.214. At present there are still some unresolved theoretical questions on the absolute normalization of the jet rates, but the outlook is promising.

One of the problems with the gluon extraction from dijet production in DIS is the background from non-gluon induced processes. This is largely avoided by measuring open charm production, which allows one to tag the boson-gluon fusion process and hence to measure the gluon density directly. The charm data in DIS have been discussed in Sec. 5.4, and the status of the theory in Sec. 3.6. Fig. 38 shows the comparison of the measurements of $F_{2}^{c \bar{c}}$ from ZEUS and H1 with the prediction using the gluon distribution extracted from the GRV94 parametrization. The agreement is very good. Clearly the errors on the measurements are still far too large to make a competitive extraction of the gluon from the charm data. Both high luminosity at HERA and improved experimental set-ups (e.g. use of Si vertex detectors) will allow considerable improvements in the future such that one should be able to measure the gluon density in the $x$ range $0.0001<x<0.01$. Open charm production in photoproduction has also been suggested as a means of measuring the gluon distribution. However it depends on the gluon density via a convolution over photon and proton parton densities, and is more likely to shed light on the former in the short term.

Measurement of the longitudinal structure function $F_{L}$ at HERA can provide a handle on the gluon distribution at low $x$. In Eq. 83 we gave the relationship between $F_{L}$ and $F_{2}$ and the gluon density, which is valid to order $\alpha_{s}$ in the coefficient functions. At $x \lesssim 10^{-3}$ the dominant contribution comes from the gluon regardless of the exact shape of the gluon distribution and we may write 23

$$
x g\left(x, Q^{2}\right)=\frac{3}{5} 5.8\left[\frac{3 \pi}{4 \alpha_{s}} F_{L}\left(0.417 x, Q^{2}\right)-\frac{1}{1.97} F_{2}\left(0.75 x, Q^{2}\right)\right]
$$

such that an accurate measurement of $F_{L}$ taken together with present measurements of $F_{2}$ should yield an accurate measurement of the gluon. This approximation is good to $\sim 2 \%$, within its own assumptions. However, heavy quark contributions and higher order corrections complicate the unfolding of the gluon density from $F_{L}$. Corrections to order $\alpha_{s}^{2}$ in the coefficient functions have heen given in ref. 215 and heavy quark contributions have been considered in ref.210. It remains true that a measurement of $F_{L}$ should be sensitive to the gluon distribution. The present data on $F_{L}$ have been given in Sec. 5.3. None of the fixed target measurements access small enough $x$ to be useful in extracting the gluon distribution. The measurement of $F_{L}$ presented by $\mathrm{H} 101$ cannot give a measurement of the gluon which is independent of the $\mathrm{H} 1$ gluon distribution as extracted from scaling violations, since the method of measurement of $F_{L}$ essentially represents a consistency check on the conventional NLO QCD fit 125 . The prospects for a future independent measurement of $F_{L}$ at HERA have been discussed in Sec. 5.3.

Inelastic $J / \psi$ photoproduction at HERA has been suggested as a process which could allow one to measure the gluon. However it appears that the perturbative calculation is not well behaved in the limit $p_{T}(J / \psi) \rightarrow 0$, and if the small $p_{T}$ region is excluded from the analvsis the predictions are not very sensitive to the small $x$ behaviour of the gluon 217. Elastic (diffractive) $J / \psi$ production in DIS and in 
Table 5. A summary of the development of the MRS ad CTEQ PDFs from 1992 to 1996, specifying the input data sets, the input $\left(Q^{2}=Q_{0}^{2}\right)$ slope of the gluon $\left(\lambda_{g}\right)$ and sea $\left(\lambda_{S}\right)$ distributions at low $x$ (the notation $\lambda$ is used when $\lambda_{g}=\lambda_{S}$ ) and the value of $\alpha_{s}$ (note this is a parameter of the global fits except for the latest MRSR sets)

\begin{tabular}{|c|c|c|c|c|}
\hline Year & $\mathrm{PDF}$ & Data Sets Used & $Q_{0}^{2}$ input & $\alpha_{s}\left(M_{Z}^{2}\right)$ \\
\hline \multirow[t]{4}{*}{1992} & $\mathrm{MRSD}^{\prime}$ & $B C D M S-F_{2}^{\mu p}\left(F_{2}^{\mu d}\right)$ & $\lambda=0\left(D 0^{\prime}\right)$ & 0.112 \\
\hline & $Q_{0}^{2}=4 \mathrm{GeV}^{2}$ & $N M C(92)-F_{2}^{\mu p}, F_{2}^{\mu d}, F_{2}^{n} / F_{2}^{p}$ & $\lambda=0.5\left(D-^{\prime}\right)$ & \\
\hline & CTEQ1 & $C C F R(93)-F_{2}^{\nu N}, x F_{3}^{\nu N}$ & low $x$ flat (1M) & 0.112 \\
\hline & $Q_{0}^{2}=2.56 \mathrm{GeV}^{2}$ & $W A 70-$ prompt $\gamma, E 605-D Y$ & low $x$ singular (1MS) & \\
\hline \multirow[t]{4}{*}{1993} & MRSH & & $\lambda=0.3$ & 0.112 \\
\hline & $Q_{0}^{2}=4 \mathrm{GeV}^{2}$ & $Z E U S(92), H 1(92)-F_{2}^{e p}$ & & \\
\hline & CTEQ2 & $(C D F-D Y)$ & $\lambda=0.258$ & 0.110 \\
\hline & $Q_{0}^{2}=2.56 \mathrm{GeV}^{2}$ & & & \\
\hline \multirow[t]{6}{*}{$1994 / 5$} & MRSA & & $\lambda=0.3$ & 0.112 \\
\hline & $\mathrm{MRSA}^{\prime}$ & $Z E U S(93), H 1(93)-F_{2}^{e p}$ & $\lambda=0.17$ & 0.113 \\
\hline & MRSG & $N A 51 A_{D Y}$ & $\lambda_{S}=0.067, \lambda_{g}=0.301$ & 0.114 \\
\hline & $Q_{0}^{2}=4 \mathrm{GeV}^{2}$ & $C D F A_{W}$ & & \\
\hline & CTEQ3 & & $\lambda=0.28$ & 0.112 \\
\hline & $Q_{0}^{2}=2.56 \mathrm{GeV}^{2}$ & & & \\
\hline \multirow[t]{6}{*}{1996} & MRSR & \multirow{6}{*}{$\begin{array}{c}Z E U S(94), H 1(94)-F_{2}^{e p} \\
E 665-F_{2}^{\mu p}\left(F_{2}^{\mu d}\right),\left(S L A C-F_{2}^{e p}\right) \\
N M C(95)-F_{2}^{\mu p}, F_{2}^{\mu d} \\
\left(C D F / D 0 \text { jet } E_{T}\right)\end{array}$} & $\lambda_{S} \neq \lambda_{g}(\mathrm{R} 1)$ & 0.113 \\
\hline & $Q_{0}^{2}=1 \mathrm{GeV}^{2}$ & & $\lambda_{S} \neq \lambda_{g}(\mathrm{R} 2)$ & 0.120 \\
\hline & & & $\lambda_{S}=\lambda_{g}(\mathrm{R} 3)$ & 0.113 \\
\hline & & & $\lambda_{S}=\lambda_{g}(\mathrm{R} 4)$ & 0.120 \\
\hline & CTEQ4 & & $\lambda_{S}=0.143, \lambda_{g}=0.206$ & 0.116 \\
\hline & $Q_{0}^{2}=2.56 \mathrm{GeV}^{2}$ & & & \\
\hline
\end{tabular}

photoproduction are more promising, since the cross-section depends on $x g\left(x, Q_{V}^{2}\right)^{2}$, where the scale of the process is given by $Q_{V}^{2}=M_{J / \psi}^{2} / 4218$. Similarly, diffractive vector meson $(\rho, \phi)$ production in DIS, at higher $Q^{2}$, depends on the square of the gluon density. These data could give information on the gluon distribution in the region $0.0001<x<0.01$. At the present time the theoretical framework for extracting the gluon distribution from these processes is still under development and the experimental precision of the data is still fairly low.

In summary, considerable progress has been made in pinning down the gluon in a wide kinematic region extending down to $x=10^{-4}$. At present the analyses from the scaling violations of the $F_{2}$ structure function are generally accepted as giving the most reliable information, since they are well understood both experimentally and theoretically (provided that the conventional NLLA is accepted as appropriate). However, further progress is expected in determinations from other processes over the next few years, such that we may expect to improve the determination of the gluon distribution over the full $x$ range.

\subsubsection{The MRSR and CTEQ4 parametrizations}

In Table 5 we summarize the development of the MRS and CTEQ parton distributions from 1992 to the 1996 sets. We specify the common data sets which were input to the fits and some of the interesting features of the parametrizations, 
such as the slope of the low $x$ gluon and sea distributions at $Q_{0}^{2}$ (where $\lambda_{g}=\lambda_{S}$ is fixed we use the common notation $\lambda$ ) and the value of $\alpha_{s}\left(M_{Z}^{2}\right)$. Note that this is a parameter of the global fit for all but the MRSR PDFs. Only the MRSR and CTEQ4 fits will be discussed in any detail.

The data sets originally used in 1992 were the older BCDMS data 21 on $F_{2}^{\mu p}, F_{2}^{\mu d}$ (note MRS only use the proton data), the $\mathrm{NMC}(92)$ data 170 on $F_{2}^{\mu p}, F_{2}^{\mu d}, F_{2}^{n / p}$ and CCFR(93) data 104 on $F_{2}^{\nu N}, x F_{3}^{\nu N}$. Both groups also supplemented this DIS data by E605 Drell Yan data and WA70 prompt photon data. When HERA(92) and HERA(93) data on $F_{2}^{e p}$ became available they were included in the fits extending the parton distributions into the low $x$ region. New non-DIS data from NA51 on the Drell Yan asymmetry, $A_{D Y} 178$, and from CDF on the $W^{ \pm}$asymmetry, $A_{W} 180$ also became available such that by 1995 one had the parametrizations MRSA', CTEQ3, valid in the regions $Q^{2} \gtrsim 4 \mathrm{GeV}^{2}, x \gtrsim 4 \times 10^{-4}$. These parametrizations still give a reasonable description of data in this kinematic region today.

However when the HERA(94) data were published in 1996 this stimulated new fits by each team: the MRSR and CTEQ4 series. New data from NMC(95) 93 and from E66597 on $F_{2}^{p}$ and $F_{2}^{d}$ were also included in these fits (note MRS do not use E665 $F_{2}^{d}$ data). In addition to this the CTEQ group include the inclusive jet measurements from CDF 205 and D0206 in their fit. MRS compare their fit to these data but they do not use them in the fit since the correct treatment of systematic errors is not clear. CTEQ drop all the prompt-photon data since the problems in fitting the $p_{T}$ spectrum for the CDF data cast doubt on our understanding of this process. MRS continue to include the lower energy WA70 prompt photon data in their fits. MRS also include SLAC data 6680 on $F_{2}^{e p}$ in their fits since they drop their starting scale to $Q_{0}^{2}=1 \mathrm{GeV}^{2}$. This change of input scale was made in response to the fact that $\operatorname{HERA}(94)$ data extend down to $Q^{2}=1.5 \mathrm{GeV}^{2}$ and to the work of various authors 219.220.38. 39 which made it apparent that DGLAP evolution could be successfully extended down to such low $Q^{2}$ values. We discuss this somewhat surprising observation further in Sec. 17. The quality of fit of the MRSR2 and CTEQ4 parametrizations to data in the kinematic range $1.5<Q^{2}<5000 \mathrm{GeV}^{2}$, $x>3 \times 10^{-5}$ is illustrated in Fig. 45 .

Both teams have allowed for variations on their basic fits. There are variants for use with LO calculations and there are variants for use in differing renormalization schemes at NLO, such as DIS rather than $\overline{\mathrm{MS}}$. There have also been variants 221 which extend below $Q^{2}=1 \mathrm{GeV}^{2}$. Such parametrizations are needed for calculating radiative corrections to higher $Q^{2}$ data as well as for making unfolding corrections to the very low $Q^{2}$ data described in Sec. 5.1. Such variants use phenomenological parametrizations of low $Q^{2}$ data adjusted to match smoothly onto the pQCD forms at higher $Q^{2}$. Variants which correspond to differing values of $\alpha_{s}$ (varying from 0.105 to 0.130 ) are also available for use in $\alpha_{s}$ determinations from data on jet production and non-DIS processes 164.222. 
Structure functions...

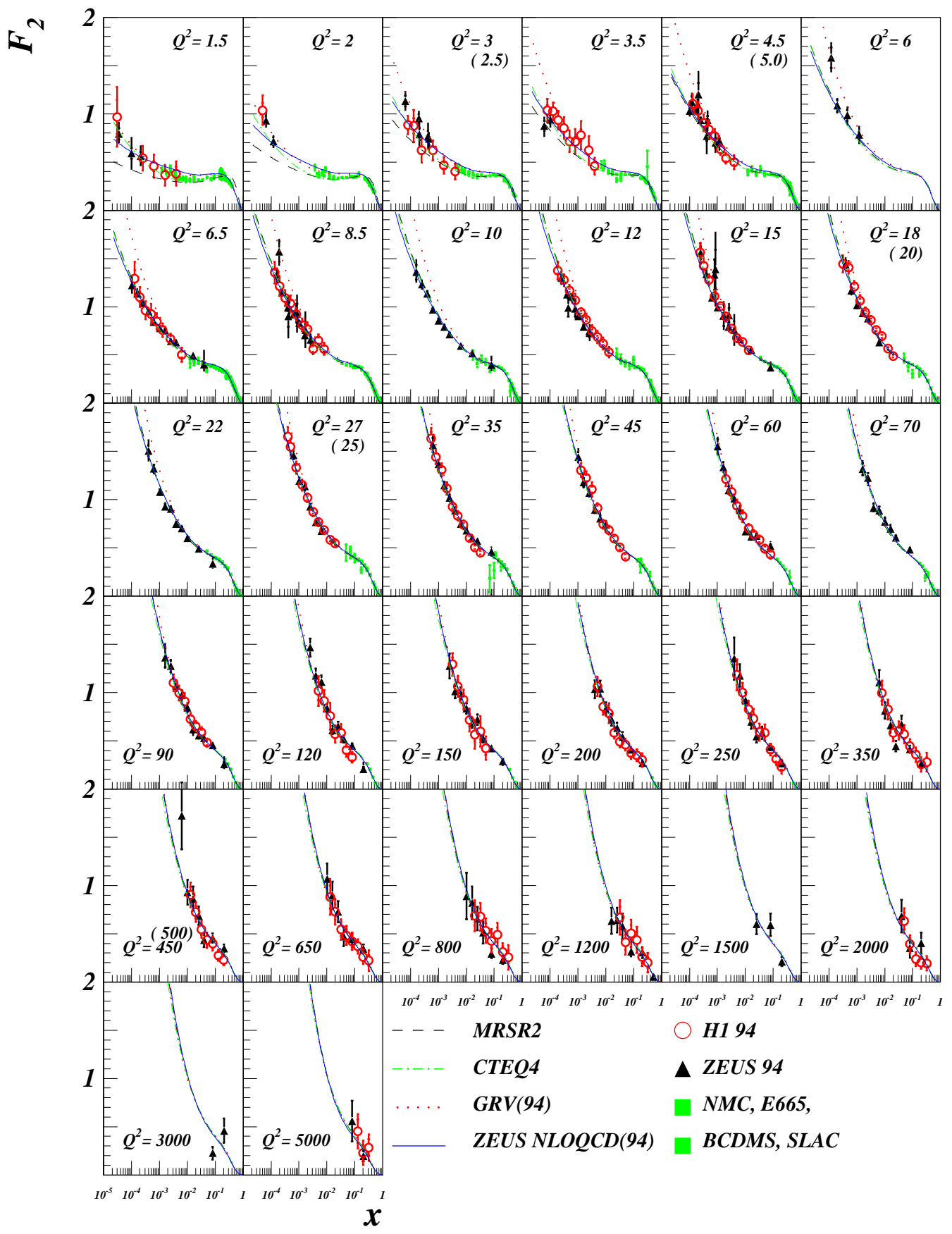

Fig. 45. ZEUS(94), H1(94), NMC(97), E665, BCDMS and SLAC $F_{2}^{p}$ data at fixed $Q^{2}$, with the MRSR2, CTEQ4 and GRV94 parton distribution functions and the ZEUS NLO QCD fit overlaid. At high $Q^{2}$ all these parametrizations are indistinguishable. 
Before the HERA data were included in the fits, the parametrizations also included variants which accounted for the possibilities of hard or soft Pomeron exchange. As noted above, the inclusion of high precision HERA data has made it possible to fit the slopes of the low $x$ gluon and sea distributions and thus to investigate the nature of the Pomeron exchange involved. The interesting theoretical possibilities which this opens up, and the limitations of conclusions drawn from fits made within the framework of conventional pQCD, are discussed in detail in Sec. 7 . Here we confine ourselves to giving a warning against over-interpreting the values of $\lambda_{g}$ and $\lambda_{S}$ which result from the fits to the parton parametrizations.

The exact values of $\lambda_{S, g}$ which give the best fit to $F_{2}$ depend on the $\epsilon_{S, g}$ values. There is a correlation between the values of $\lambda$ and $\epsilon$ such that a more negative $\epsilon$ reduces the value of $\lambda$ and a more positive value increases it. One can fit the same data with different $\epsilon_{S}$ values (and $\epsilon_{g}=0$ ) such that $\lambda_{g}=\lambda_{S}=0.2, \epsilon_{S}=-3.3$ or $\lambda_{g}=\lambda_{S}=0.3, \epsilon_{s}=-1.1$ or $\lambda_{g}=\lambda_{S}=0.4, \epsilon_{S}=+2.9$ and all these possibilities give similar fit quality. Hence the same data can appear in need of more or less singular input depending on the exact form of the parametrization. MRSA has $\lambda_{S}=\lambda_{g}=0.3, \epsilon_{S}=-1.1, \epsilon_{g}=0$, whereas MRSA' has $\lambda_{S}=\lambda_{g}=0.17, \epsilon_{S}=$ $-2.55, \epsilon_{g}=-1.9$. The minimal forms which have $\epsilon_{S, g}=0$ give a better guide to the low $x$ behaviour if one only considers the value of $\lambda_{S, g}$.

The MRSR1,2 fits are done for $\lambda_{g} \neq \lambda_{S}$ and fixed $\alpha_{s}=0.113,0.120$ respectively. The reasons for this choice of $\alpha_{s}$ values is discussed in Sec. 6.2. The best overall fit is MRSR2, which has $\alpha_{s}=0.120$ and $\lambda_{S}=0.15, \epsilon_{S}=1.1, \lambda_{g}=-0.51, \epsilon_{g}=-4.2$, at $Q_{0}^{2}=1 \mathrm{GeV}^{2}$. These values are not directly comparable to the previous MRS $\lambda$ values because of the drop in the starting scale. One notes that the gluon low $x$ slope has become valence-like, however this quickly changes as $Q^{2}$ increases, such that $\lambda_{S}$ and $\lambda_{g}$ become equal (to $\sim 0.2$ ) at $Q^{2} \sim 5 \mathrm{GeV}^{2}$ (as in the MRSA' distributions) and for larger $Q^{2}, \lambda_{g}>\lambda_{S}$, as expected by pQCD, see Fig. 46 .

Table 6 gives $\chi^{2}$ per data point for the parametrizations CTEQ3, CTEQ4, MRSA', MRSR1 and MRSR2 for the DIS data, and some of the relevant non-DIS data. The numbers of parameters differ between the parametrizations: CTEQ3, CTEQ4, MRSA', MRSR1, MRSR2 have 15,18,17,19,19 shape parameters respectively, in addition to the relative normalizations of the data sets, and the value of $\alpha_{s}$ when it is fitted rather than fixed. We also present the $\chi^{2}$ per degree of freedom for fits to the DIS data in the final row so that we can compare the goodness of fit of each of these parametrizations to current data.

When comparing these $\chi^{2}$ values one should bear in mind that it is not really possible to define a one standard deviation covariance matrix of uncertainties on these parton distribution functions. Firstly, because the correlations between experimental systematic errors are not easily taken into account. Secondly because there are theoretical uncertainties, such as the treatment of heavy quarks, the treatment of scale uncertainties when dealing with non-DIS data, the choice of $Q_{0}^{2}$ and of the form of the input parametrization, which are not easy to quantify in terms of a correlated systematic uncertainty. A related issue arises when using PDFs as input 

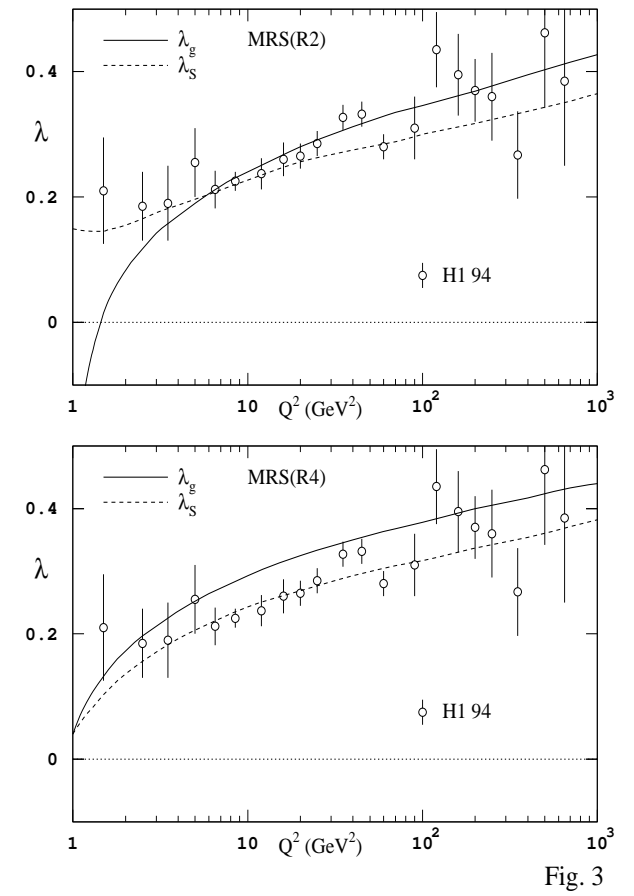

Fig. 46. The evolution of the parameters $\lambda_{S}$ and $\lambda_{g}$ with $Q^{2}$, for the MRSR2 and MRSR4 parametrizations. The evolution of the $\lambda$ parameter as evaluated from H1(94) data is also shown. Note that these parameters are not evaluated in exactly the same way. For $Q^{2}>Q_{0}^{2}$ the MRSR PDFs are not given by the analytic forms of Eq. 142. Hence to quote a value of $\lambda$ as a function of $Q^{2}$ the authors perform fits of the evolved distributions to forms of this type for each value of $Q^{2}$ which is of interest. The H1 $\lambda$ parameter is evaluated by the simpler procedure of fitting the form $x^{-\lambda}$ to $F_{2}$ data in the low $x$ range, $x<0.1$, for various $Q^{2}$ bins. The mean value of $x$ at which this evaluation is made will increase with $Q^{2}$. These different procedures this will not give fully comparable $\lambda$ values

for calculations of high energy scattering processes in order to constrain the parameters of the Standard Model, or even to investigate physics beyond the Standard Model. The systematic uncertainty on a result coming from the choice of PDF is not easily quantified since the best global fits are derived from similar assumptions. We need to consider variants on the standard PDFs in which the parameters most sensitive to the derived result are varied as widely as possible, while maintaining a reasonable global fit quality taking into account the systematic errors on the input data. For an interesting discussion of these issues see ref. 203.223 .224$.

Both the MRS team 4525 and the CTEQ team 44 have recently updated their parton distributions to account for heavy quark production consistently from the threshold region to the asymptotic region. The new CTEQ parton distributions, CTEQ4HQ, were produced by fitting to the same data sets as the CTEQ4 series to aid comparison. They are similar to the standard CTEQ4M set but the fit quality is somewhat improved, $\chi^{2} / n d f=1.03$ especially for low $x$ data. The MRS team have included the new $\mathrm{NMC}(97)$ data 95 in their (MRRS) analysis 45 . The fit quality 
Table 6. $\quad \chi^{2}$ per data point for the parametrizations CTEQ3, CTEQ4, MRSA', MRSR1 and MRSR2 for the DIS data, and relevant non-DIS data, including the CDF/D0 jet data, for which systematic errors have not been included. Note that the $\chi^{2}$ values are worked out for all the data sets using the published forms of the parametrizations and the CTEQ evolution programme for all the quoted values except those of MRSR, which were supplied by the authors. Not all of the data sets included were available at the time when the earlier parametrizations were issued, the $\chi^{2}$ are worked out retrospectively. The number of data points quoted differs for MRSR1, MRSR2, since these have a lower starting value $Q_{0}^{2}=1 \mathrm{GeV}^{2}$. The other parametrizations have their $\chi^{2}$ values worked out from the CTEQ starting point $Q^{2}=2.56 \mathrm{GeV}^{2}$. Some of the $\chi^{2}$ values for MRSR fits to non-DIS data are estimated since the authors indicate that they are similar in quality to those achieved with MRSA and MRSA'. The WA70 $\chi^{2}$ for MRSA' is estimated as equal to that for MRSA. The $\chi^{2}$ per degree of freedom given in the final row includes only the DIS data.

\begin{tabular}{cccccc}
\hline Data & CTEQ3 & MRSA $^{\prime}$ & CTEQ4 & MRSR1 & MRSR2 \\
\hline$B C D M S F_{2}^{p}$ & $130 / 168$ & $157 / 168$ & $145 / 168$ & $265 / 174$ & $320 / 174$ \\
$B C D M S F_{2}^{d}$ & $187 / 156$ & $214 / 156$ & $186 / 156$ & & \\
$C C F R(93) F_{2}$ & $69 / 63$ & $68 / 63$ & $83 / 63$ & $41 / 66$ & $56 / 66$ \\
$C C F R(93) x F_{3}$ & $41 / 63$ & $54 / 63$ & $47 / 63$ & $51 / 66$ & $47 / 66$ \\
$N M C r a t i o$ & $133 / 89$ & $143 / 89$ & $131 / 89$ & $136 / 85$ & $132 / 85$ \\
$N M C(95) F_{2}^{p}$ & $147 / 104$ & $129 / 104$ & $97 / 104$ & $155 / 129$ & $147 / 129$ \\
$N M C(95) F_{2}^{d}$ & $137 / 104$ & $152 / 104$ & $93 / 104$ & $139 / 129$ & $129 / 129$ \\
$Z E U S(94) F_{2}^{p}$ & $549 / 179$ & $369 / 179$ & $243 / 179$ & $326 / 204$ & $308 / 204$ \\
$H 1(94) F_{2}^{p}$ & $220 / 172$ & $150 / 172$ & $119 / 172$ & $158 / 193$ & $149 / 193$ \\
$E 665 F_{2}^{p}$ & $48 / 35$ & $38 / 35$ & $41 / 35$ & $62 / 53$ & $63 / 53$ \\
$E 665 F_{2}^{d}$ & $45 / 35$ & $29 / 35$ & $32 / 35$ & & \\
$S L A C F_{2}^{p}$ & & & & $108 / 70$ & $95 / 70$ \\
$C D F A_{W}$ & $3 / 9$ & $3.7 / 9$ & $4.3 / 9$ & $\sim 4 / 9$ & $\sim 4 / 9$ \\
$N A 51 A_{D} Y$ & $0.4 / 1$ & $0.1 / 1$ & $0.6 / 1$ & $\sim 0.1 / 1$ & $\sim 0.1 / 1$ \\
$E 605$ & $93 / 119$ & $94 / 119$ & $98 / 119$ & $\sim 95 / 119$ & $\sim 95 / 119$ \\
$W A 70$ & $23 / 39$ & $\sim 21 / 39$ & & & \\
$C D F j$ jet & & & & $222 / 24$ & $52 / 24$ \\
\hline total $\chi^{2} / n d f$ & 1.49 & 1.31 & 1.06 & 1.33 & 1.32 \\
\hline
\end{tabular}

has improved, $\chi^{2} / n d f=1.19$ (compared to $\chi^{2} / n d f=1.25$ for MRSR2 on the same data) especially at low $x$, and the corresponding value of $\alpha_{s}, \alpha_{s}\left(M_{Z}^{2}\right)=0.118$ also gives a better fit to BCDMS data. The resulting description of HERA and EMC charm data is good. More recently 225 MRS have also included the updated $\operatorname{CCFR}(97)$ data 100 in their fits (excluding the contentious region $x<0.1$ ). This does not change the fit quality and fit parameters significantly compared to MRRS. The resulting parton distributions are very similar to those of CTEQ4HQ when evolved to $Q^{2}=25 \mathrm{GeV}^{2}$.

\subsubsection{Dynamically generated partons: the GRV parametrization}

Whereas the parametrizations provided by the MRS and CTEQ groups depend crucially on the nop-perturbative input parametrization at $Q_{0}^{2}$, the parametrizations of the GRV group 383 are far less dependent on their inputs.

The original idea behind these parametrizations is that at some VERY LOW scale $Q^{2}=\mu^{2}$ the nucleon consists only of constituent valence quarks. As $Q^{2}$ increases, one generates the gluons and sea quarks in the nucleon dynamically from 
these valence quarks, through the conventional DGLAP equations for the processes $q \rightarrow q g, g \rightarrow q \bar{q}$. It did not prove possible to describe all relevant data (in particular the gluon distributions required by the prompt photon data) using such a picture, and it was accordingly modified to include gluons and sea quarks at the starting scale $\mu^{2}$, BUT these distributions have a valence-like shape (i.e. small at small $x$ ). One may even interpret this picture by saying that the gluons and sea quarks are frozen upon the valence current quarks for $Q^{2}<\mu^{2}$, and these composite objects form the constituent quarks 39 .

The valence quark distributions are fixed by taking the valence parametrizations from a conventional fit, such as MRSA, at $Q_{0}^{2}=4 \mathrm{GeV}^{2}$ and evolving them

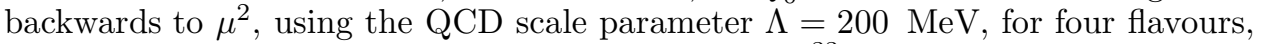
matching $\alpha_{s}$ at flavour thresholds in the usual way 33 . The valence-like sea and gluon distributions at $\mu^{2}$ are parametrized by simple forms like

$$
x g\left(x, \mu^{2}\right)=A x^{\alpha}(1-x)^{\beta}, \quad x \bar{q}\left(x, \mu^{2}\right)=A^{\prime} x^{\alpha^{\prime}}(1-x)^{\beta^{\prime}}
$$

where the free parameters are set by fitting to fixed target-DIS and prompt photon data, after $Q^{2}$ evolution from the starting scale $\mu^{2}$ back up to the $Q^{2}$ values of the relevant data sets has been performed (i.e. the gluon distribution at any scale $Q^{2}>\mu^{2}$ consists of the original gluon distribution at $\mu^{2}$ augmented by the gluons which evolve from the valence quarks). The scale $\mu^{2}$ is set as the scale at which the gluon distribution is as hard as the valence quark distribution $u_{v}$. The splitting $q \rightarrow q g$ naturally generates a softer distribution in the split products than that of the original quark, hence a gluon distribution which is harder than the $u_{v}$ distribution is considered physically unreasonable. Thus the scale $\mu$ at which $g \sim u_{v}$ is assumed to be the lowest scale at which the DGLAP equations may be used.

The original predictions of GRV (GRV91) 38 were a little too steep to describe the HERA low $x$ data, a better fit was obtained (GRV94) 39 by modifications which took into account more modern fixed target data to fix the input distributions, and by treating the heavy quark component of $F_{2}$ in a more sophisticated manner 40 , using a fixed flavour number (FFN) scheme. This essentially uses 3-flavours of massless parton and generates the charm contribution to $F_{2}$ through the boson-gluon fusion process.

It turns out that $\mu^{2} \sim 0.3 \mathrm{GeV}^{2}$, which seems a very low scale at which to use perturbative QCD. However, GRV argue that it is the value of $\alpha_{s}\left(\mu^{2}\right) / \pi \sim 0.2$, which determines the relative size of higher order corrections, not the value of $\alpha_{s}\left(\mu^{2}\right)$, and that their predictions are perturbatively stable between LO and NLO if one considers measurable quantities like structure functions, rather than parton distributions. They emphasize that their calculation is only applicable to the leading twist operators of $\mathrm{QCD}$, and clearly at very low $Q^{2}$ higher twist operators can be important. Hence GRV state that the calculation can be made consistently at low $Q^{2}$, but they only expect the resulting parametrization to describe reality for somewhat higher $Q^{2}\left(\gtrsim 0.6 \mathrm{GeV}^{2}\right)$. The very recent low $Q^{2}$ data from HERA indicate that this expectation is correct, GRV predictions begin to describe the data qualitatively, for $Q^{2}$ values, $Q^{2} \gtrsim 0.8 \mathrm{GeV}^{2}$, as illustrated in Fig. 45 and Fig. 84. 
However one can also see that the GRV94 parametrization lies systematically above the lowest $x$ data for moderate $Q^{2}$ values. Note that since HERA data were not input to the GRV94 parametrization it represents a prediction for the behaviour of $F_{2}$ in the HERA region. Whereas at larger $x, x>0.01$, the approach is similar to that of MRS and CTEQ, at small $x, x<0.01$, it loses sensitivity to the form of the initial distributions at $Q^{2}=\mu^{2}$. This is because of the long evolution length between $\mu^{2}$ and the $Q^{2}$ values at which we compare with data, taken together with the fact that the initial distributions are valence-like. The resulting gluon and sea quark distributions are very steep at small $x$ for large $Q^{2}$, but they flatten as $Q^{2}$ decreases, and this clearly describes the trend of the HERA low $x$ data qualitatively. Such behaviour is characteristic of the predictions of pQCD at low $x$. We discuss this in detail in Sec. ․

\subsection{Determinations of $\alpha_{s}\left(M_{Z}^{2}\right)$}

The simplest predictions of pQCD concern the $Q^{2}$ evolution of the non-singlet structure functions. In principle one can extract a value of $\Lambda$, and hence a value of $\alpha_{s}$, from such data independent of assumptions as to the shape of the nonsinglet quark distributions. However the early data on non-singlet quantities (such as $x F_{3}$ in neutrino scattering and $F_{2}^{p}-F_{2}^{n}$ in muon scattering) were insufficiently accurate for precision determinations. Hence $\alpha_{s}$ determinations from deep inelastic scattering data were done within the global fits to singlet and non-singlet structure functions, in which the parton distributions are fitted at the same time as $\alpha_{s}$. There is then a coupling between the value of $\Lambda$ extracted and the shape of the gluon distribution, which is most easily understood from the LO DGLAP equations (Eqs. 61, 62. Increasing $\Lambda$ increases the negative contribution from the $P_{q q}$ term, but this may be compensated by the positive contribution from the $P_{q g}$ term if the gluon is made harder. The global fits of the MRS and CTEQ teams address this problem by looking at data from other physical processes to tie down the gluon distribution.

Before the most recent high precision HERA(94) data were included in these fits the $\alpha_{s}$ values extracted were in the region of $\alpha_{s} \sim 0.113$. This contrasts with the values of $\alpha_{s} \sim 0.120$ extracted from LEP data from the $n$-jet production rates and the hadronic width of the $Z^{0} 226$. It also seems that the recent CDF data on the single jet inclusive $E_{T}$ distribution is best described by $\alpha_{s} \sim 0.120$. Much thought has gone into understanding the origins of this discrepancy including the suggestion that new physics maybe responsible for a process dependent discrepancy. However, when one looks more closely at the theoretical uncertainties involved in the extractions (as well as at the experimental statistical and systematic errors) it is not clear that there is any significant discrepancy. The reviews of Bethke 227 and Stirling 228 summarize the latest measurements and calculations of theoretical uncertainty for $\alpha_{s}$ extractions from non-DIS processes.

In the present section we discuss the uncertainties on extractions of $\alpha_{s}$ from 
Table 7. A summary of the results for $\alpha_{s}\left(M_{Z}^{2}\right)$ extracted from structure function data. The experimental errors include both statistical and systematic errors. The treatment of correlated errors and the methods used by different authors are discussed in the text

\begin{tabular}{|c|c|c|c|}
\hline Data & $\alpha_{s}\left(M_{Z}^{2}\right)$ & Authers & Method \\
\hline BCDMS/SLAC $F_{2}$ & $0.113 \pm 0.003(e x p) \pm 0.004(t h)$ & VM得 & DGLAP NLO \\
\hline $\operatorname{CCFR}(93) x F_{3}$ & $0.107 \pm 0.006(\exp )$ & CCFR些 & DGLAP NLO \\
\hline $\operatorname{CCFR}(93) F_{2}+x F_{3}$ & $0.111 \pm 0.004(\exp )$ & $\mathrm{CCFR} \stackrel{104}{=}$ & DGLAP NLO \\
\hline $\operatorname{CCFR}(97) x F_{3}$ & $0.118 \pm 0.006(\exp )$ & CCFR & DGLAP NLO \\
\hline $\operatorname{CCFR}(97) F_{2}+x F_{3}$ & $0.119 \pm 0.004(\exp )$ & CCFR & DGLAP NLO \\
\hline $\operatorname{CCFR}(97) F_{2}+x F_{3}$ & $0.119 \pm 0.002(e x p) \pm 0.004(t h)$ & CCFR 106 & DGLAP NLO \\
\hline Global GLS & $0.108_{-0.006}^{+0.005}(\exp ) \pm_{0.006}^{0.004}(t h)$ & $\mathrm{CCFR}=42$ & sum rule NNNLO HT \\
\hline CCFR GLS(97) & $0.112_{-0.009}^{+0.007}(\exp ) \pm 0.009(t h)$ & CCFR & sum rule NNNLO HT \\
\hline HERA(94) in global & $0.1146 \pm 0.0036(\exp )$ & Alekhinthy & Bayesian \\
\hline $\operatorname{HERA}(93)$ & $0.120 \pm 0.005(\exp ) \pm 0.009(t h)$ & $\mathrm{BF}^{245}$ & DGLAP NLO \\
\hline $\mathrm{H} 1(94)$ & $0.122 \pm 0.004(e x p) \pm 0.007(t h)$ & $\mathrm{BF} 247$ & DGLAP NLO \\
\hline
\end{tabular}

structure function data ${ }^{\mathrm{r}}$ paying particular attention to the estimation of experimental and theoretical uncertainties. We first consider the evaluations of $\alpha_{s}$ from fixed target data. We concentrate on analyses of data from individual experiments rather than global fits, since the systematic errors can then be specified properly. We then consider the impact of the HERA data including the further theoretical uncertainties which are relevant for small $x$ data. The results for $\alpha_{s}$ are presented in Table 7, where statistical and systematic errors are combined into a single experimental error and an estimate of the theoretical uncertainty is also given, where available.

\subsubsection{Determinations of $\alpha_{s}$ from fixed target data}

Two main methods are used: analysis of scaling violations and of sum rules. Analyses of scaling violation data are most usually done at NLO by numerical solution of the DGLAP equations. The experimental uncertainties on such measurements are now dominated by the systematic errors coming from energy scale uncertainties. For a discussion of possible future improvements see ref. 203. It is clear that taking into account correlations between systematic errors can lead to a significant reduction in the total systematic error 231. However, theoretical uncertainties are just as important. Blümlein et al 232 have considered some of the theoretical uncertainties involved in extracting $\alpha_{s}$ from such fits. Differences arise from various sources. There are different approximations to the solution of Eq. 60 for $\alpha_{s}$ at second order and there are different ways of dealing with the behaviour of $\alpha_{s}$ at the heavy quark thresholds (see Sec. 3.6) and these choices give an uncertainty of \pm 0.001 . One may choose to perform the evolution in Mellin space or in $x$ space and this choice leads to an uncertainty of \pm 0.003 . Scheme uncertainty seems to

\footnotetext{
$\mathrm{r}^{\mathrm{r}}$ We shall not discuss the use of $n+1$ jet rate in DIS measurements to measure $\alpha_{s}$, since there are still some unreso theoretical questions on the absolute malizations of the jet rates. For latest results see ref.229, for a review of the technique see ref. 230 .
} 
give the largest contribution. Considering the correct scale for the evaluation of $\alpha_{s}$ to range from $Q^{2} / 4$ to $4 Q^{2}$ gives an uncertainty of ${ }_{-0.006}^{+0.004}$ from renormalization and \pm 0.003 from mass factorization even when $Q^{2}$ is restricted to $Q^{2}>50 \mathrm{GeV}^{2}$. The scheme dependence should be considerably reduced if one could work to NNLO. Small $x$ data is particularly sensitive to the need for higher order corrections. One should also consider the uncertainty coming from restricting the analysis to the conventional leading $\ln Q^{2}$ approximations. There can be important corrections of the form $\alpha_{s} \ln (1-x)$ at high $x$ and $\alpha_{s} \ln (1 / x)$ at small $x$. Presently the highest $x$ data come from the BCDMS experiment, and their major contribution is in the region $0.3<x<0.5$, such that high $x \operatorname{logs}$ are not likely to be important. However the HERA data do access small enough $x$ that $\ln (1 / x)$ corrections could be important.

One of the earliest analyses which accounted for theoretical uncertainties was given by Virchaux and Milsztajn 147 using BCDMS and SLAC $F_{2}$ data. This analysis accounted for the correlated systematic errors in the BCDMS data, the relative normalizations of the data sets, and the need for target mass corrections and higher twist contributions at high $x$, low $Q^{2}$. Theoretical uncertainties from the choice of the renormalization and factorization scales, the position of the flavour thresholds, and the value of $R$ were also considered. The quoted value is very insensitive to the uncertainties on the gluon distribution, since a substantial part of the data lies at high $x, x>0.25$, where the non-singlet structure function dominates.

Accurate data are now available on non-singlet quantities. In 1993 CCFR gave an NLO QCD analysis of their non-singlet $x F_{3}$ data 104 for $Q^{2}>10 \mathrm{GeV}^{2}$ - large enough that higher twist effects are considered negligible. The analysis was also done using $F_{2}$ data instead of $x F_{3}$ data for $Q^{2}>15 \mathrm{GeV}^{2}, x>0.5$, where $F_{2}$ is dominantly non-singlet. (However, the increased precision gained by using higher statistics $F_{2}$ data, is partly negated by increased theoretical uncertainty in using a quantity which is not strictly a non-singlet.) These analyses gave low values of $\alpha_{s}$ in agreement with the BCDMS value. In 1997 CCFR updated their data106 as detailed in Sec. 5.2. Fits have again been made to $x F_{3}$ and $x F_{3}+F_{2}(x>0.5)$ data under the same $Q^{2}$ cuts as for the 1993 analysis, and the values of $\alpha_{s}$ extracted are both significantly higher. If the correlations between systematic errors are included in the fits then a reduced systematic error results, such that CCFR quote the final value $\alpha_{s}=0.119 \pm 0.002(\exp ) \pm 0.004(t h)$, where the theoretical uncertainty includes accounting for higher twist corrections and renormalization and factorization scale uncertainties. We present the values from both the updated and the older data to aid comparison with the analyses of other authors where the older data was used.

As an illustration of how different analysis techniques and different assumptions can affect the results extracted for $\alpha_{s}$, we briefly review some independent analyses of the CCFR data. Kataev et al 233 (KKSP) have used the alternative technique of reconstruction of the structure function from its Mellin moments, using an expansion in terms of Jacobi polynomials 234, in the same kinematic regions as defined by CCFR(93). The main advantage of this method is that NNLO corrections can be included. In practice the inclusion of NNLO correction in this analysis does not change 
the central values for $\alpha_{s}$ significantly (e.g. $\alpha_{s}=0.109 \pm 0.006(\exp ) \pm 0.003(t h)$ for the $x F_{3}$ data) although it reduces theoretical uncertainties due to scheme dependence. Target mass corrections were included in the KKSP analysis but dynamical higher twist contributions were not. Sidorov 151 has also used the Jacobi polynomial method to analyse the CCFR $(93) x F_{3}$ data, including higher twist terms by adding a term $h(x) / Q^{2}$ to the leading twist expressions for $x F_{3}$, and including data in the $x, Q^{2}$ range $0.015<x<0.65,1.3<Q^{2}<501 \mathrm{GeV}^{2}$, rather than cutting out the low $Q^{2}$ data. The value of $\alpha_{s}$ extracted is then even lower than that extracted by KKSP, $\alpha_{s}=0.104_{-0.008}^{+0.006}$. This analysis has also been used for a combined fit to world neutrino data from other collaborations 153 . The data from CDHS 235, SKAT 236, BEBC-WA59 150, BEBC-GGM237 and the new JINR-IHEP 238 data on $x F_{3}$ were analysed in the kinematic region $0.03<x<0.80,0.5<Q^{2}<196 \mathrm{GeV}^{2}$ (with a cut of $x>0.35$ on CDHS data to account for known problems 117). This global analysis also yields a very low value for $\alpha_{s}, \alpha_{s}=0.107 \pm 0.003$. More recently KKSP have updated their NNLO analysis to consider the $\operatorname{CCFR}(97) x F_{3}$ data including consideration of higher twist effects 152,239. Using the renormalon prediction for the higher twist correction 31 they obtain $\alpha_{s}=0.117 \pm 0.006(\exp ) \pm 0.003(t h)$ but note that this should be compared with $\alpha_{s}=0.121 \pm 0.006(\exp ) \pm 0.006(t h)$ at NLO from their own NLO analysis, rather than that of CCFR, and that they do not take into account correlations between systematic errors. Tokarev and Sidorov 240 have also used the Jacobi polynomial method to analyse the updated CCFR(97) $x F_{3}$ data, including target mass, higher twist and nuclear corrections, and they quote a value of $\alpha_{s} \sim 0.112$, much lower than the values from the corresponding analyses of CCFR or KKSP. In contrast, Shirkov et al 241 have made an analysis of the same data accounting for heavy quark thresholds, and they obtain, $\alpha_{s}=0.122 \pm 0.004$. Thus the theoretical error on $\alpha_{s}$ quoted by the CCFR collaboration from their CCFR(97) analysis may be somewhat underestimated.

Data on the GLS sum rule can also be used to extract $\alpha_{s}$. The GLS sum rule is a prediction for the $n=1$ moment of the non-singlet structure function $x F_{3}$ which has been worked out to NNNLO

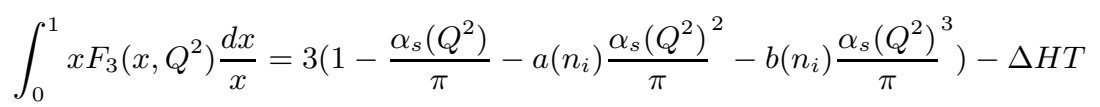

where $a, b$ are known calculable functions of the appropriate number of flavours $n_{i}$, and $\Delta H T$ represents the higher twist contribution. Higher twist contributions to the low $n$ moments are much more reliably estimated than contributions to the structure functions. The higher twist contribution to the GLS sum rule has been estimated by several different techniques 28 to be $0.27 \pm \frac{0.14}{Q^{2}}$. This constitutes the major theoretical uncertainty on the measurement. The major experimental uncertainty comes from the need to extrapolate to $x \rightarrow 0$ in order to perform the integral. Accordingly, the CCFR collaboration made a measurement using their own $\operatorname{CCFR}(93)$ data and that of BEBC-WA59, WA25, GGM, SKAT and FNAL-E180 (and some SLAC data on $F_{2}$ for $x>0.5$ ), to achieve the best possible kinematic coverage 242. One can measure the value of the sum for various different $Q^{2}$ values, and thus determine $\alpha_{s}\left(Q^{2}\right)$ as a 
function of $Q^{2}$, in the low $Q^{2}$ range where most of the data lie, $1<Q^{2}<20 \mathrm{GeV}^{2}$. Since $\alpha_{s}\left(Q^{2}\right)$ is changing rapidly in this region, such an analysis should be more sensitive than those made at higher $Q^{2}$. The equivalent value of $\alpha_{S}\left(M_{Z}^{2}\right)$ extracted is low ${ }^{\text {s }}$ (see Table 7). However, we note that Chyla and Kataev 243 (CK) have made an independent analysis of the data on the GLS sum rule from the CCFR(93) data alone 105 , paying particular attention to uncertainties due to scheme dependence, and they obtain the higher value $\alpha_{s}=0.115 \pm 0.005(\exp ) \pm 0.003(t h)$. Finally, CCFR have recently updated their GLS analysis 24 to use the updated CCFR(97)data, in the region $0.02<x<0.5$. A power law fit is used to make the extrapolations into the remaining $x$ region (where other $\nu N$ DIS data was used to determine the parameters of this fit). Their preliminary value for $\alpha_{s}$ from this GLS analysis is larger than the corresponding result on 93 data (see Table 7), but it is still lower than the result from the DGLAP NLO fit to the scaling violations.

\subsubsection{Determinations of $\alpha_{s}$ from HERA data}

We will now consider how the HERA data have contributed to our knowledge of $\alpha_{s}$. We have already discussed the global fits of MRS and CTEQ in Sec. 6.1. The purpose of these fits is to extract the parton distributions rather than to measure $\alpha_{s}$. Thus in the latest MRSR fits distributions are given for fixed values of $\alpha_{s}$ : low, $\alpha_{s}=$ 0.113 , and high, $\alpha_{s}=0.120$. We note that in these fits the HERA(94) data prefer the latter value, in contrast to the fixed target data (not including the CCFR(97) data) which prefer the former. The CTEQ4 analysis also uses the somewhat higher value of $\alpha_{s}=0.116$ to accommodate the HERA(94) data. However, the correlations between experimental systematic errors are not accounted for in these global fits. Alekhin 191 has made a new global fit to the high precision DIS data from BCDMS, the latest $\mathrm{NMC}(97)$ data and H1(94) and ZEUS(94) data, for which information on correlations is available. He takes account of point to point correlations using a Bayesian treatment of systematic errors. The data is cut such that $W^{2}>16 \mathrm{GeV}^{2}$, $Q^{2}>9 \mathrm{GeV}^{2}$ to reduce sensitivity to higher twist contributions. He makes target mass corrections and accounts for heavy quarks using the GRV formalism. The fit is made with $\alpha_{s}$ and the parametrization of the parton distributions as free parameters and the error quoted on $\alpha_{s}$ fully accounts for its correlation to the shape of the gluon distribution. The value of $\alpha_{s}$ extracted is $\alpha_{s}=0.1146 \pm 0.0036$.

It is also interesting to consider the HERA data alone rather than within a global fit. Ball and Forte 245 predict that structure functions in the small $x$, high $Q^{2}$ limit exhibit 'Double Asymptotic Scaling' (DAS), which we discuss in Sec. 7. One may fit directly to the DAS predictions for the structure functions in order to extract $\alpha_{s}$, and such a fit to H1(94) data yields $\alpha_{s}=0.113 \pm 0.002$ (stat) \pm 0.007 (sys) 24 , but such a procedure can only be approximate since the DAS formulae are merely good approximations to the full NLO QCD predictions. Ball and Forte have made

\footnotetext{
s Amore recent evaluation of the GLS sum rule using the new data from the JINR-IHEP detector 238 yields a much larger value of $\alpha_{s}\left(M_{Z}^{2}\right), \alpha_{s} \sim 0.120$, but the statistical and systematic errors on these data are still rather large.
} 
an NLO QCD fit tailored to low $x$ HERA data in order to measure $\alpha_{s}$. They take a set of parton distributions such as MRSR, known to fit data at high $x$, evolve them to a starting scale $Q_{0}^{2}$ (which is fixed in any one fit, but choices are varied in the range $1<Q_{0}^{2}<25 \mathrm{GeV}^{2}$ ) and then cut off their low $x$ tails for $x \leq 0.01$, replacing them with new tails: $x q \sim x^{-\lambda_{S}}$ for singlet quarks (at the low $x$ values considered the singlet quark distribution may be considered as identical to the sea quark distribution) and $x g \sim x^{-\lambda_{g}}$ for the gluon, where the $\lambda$ values are parameters of the fit. These new distributions (together with unmodified nonsinglet distributions) are then evolved to the $Q^{2}$ values of the HERA(93) data, using a value of $\alpha_{s}$ which is also a parameter of the fit.

The results of the best fit (see Table 7) show that $\alpha_{s}$ and the small $x$ shape of the gluon distribution, characterized by the parameter $\lambda_{g}$, are correlated as usual, BUT the shape of the gluon and the singlet quark distribution, characterized by the parameter $\lambda_{S}$, are also strongly correlated in this kinematic region and the singlet quark distribution is strongly constrained directly through the measurement of $F_{2}$. Thus the errors on $\alpha_{s}$ are smaller at low $x$ than at moderate $x$ because at small $x$ the gluon is 'driving' the behaviour of the singlet quark distribution. The theoretical uncertainties from sources such as scheme dependence are discussed in some detail, with similar conclusions to those of Blümlein232. However Ball and Forte go further and consider the need for $\ln (1 / x)$ corrections to the conventional leading $\ln Q^{2}$ expansion. The necessary modifications to the conventional expansion scheme will be discussed in Sec. 7.4, here we quote only the result that the uncertainty on $\alpha_{s}$ from this source is ${ }_{-0.006}^{+0.002}$, asymmetric because the central value of a fit including $\ln (1 / x)$ contributions is $\alpha_{s}=0.115$. An update to this analysis using the H1(94) data suggests that this uncertainty has now been reduced since fits including $\ln (1 / x)$ corrections are no longer competitive in $\chi^{2}$ to the conventional fits 247 .

However, Thorne 248 disagrees with the conclusion that $\ln (1 / x)$ terms are not necessary to fit the low $x$ data. This will be discussed in detail in Sec. 7.4. The consequence for the extraction of $\alpha_{s}$ is that low $x$ data cannot give a reliable estimate of $\alpha_{s}$ when treated conventionally. This is because a fully renormalization scheme consistent perturbative expansion necessitates the inclusion of $\ln (1 / x)$ terms. Unfortunately, this can only be done to leading order at the moment, due to the lack of full knowledge of the $\ln (1 / x)$ terms to next-to-leading order. Thus a fit to $\alpha_{s}$ at NLO cannot be consistently performed in regions of $x$ where $\ln (1 / x)$ terms are important. He estimates this region as $x \lesssim 0.01$. Thus conventional extractions of $\alpha_{s}$ using the HERA data are unreliable. When the next-to-leading $\ln (1 / x)$ terms are fully known an extraction which does not suffer from uncertainties due to scheme dependence can be made. Thorne's provisional value for a properly extracted value of $\alpha_{s}$ is $\sim 0.115249$.

A further reservation on the use of low $x$ data in conventional fits comes from the uncertainties due to the treatment of heavy quark thresholds. None of the analyses discussed above have treated heavy quark production consistently in all kinematic regions. A correct treatment of charm production will make a significant difference 
at small $x$ where $F_{2}^{c \bar{c}}$ is a greater fraction of $F_{2}$. If a QCD fit is made without accounting for the charm threshold correctly then threshold behaviour can fake a stronger dependence of $F_{2}$ on $Q^{2}$ than is truly attributable to QCD scaling violations and thus make the value of $\alpha_{s}$ seem larger. For example, the MRRS analysis discussed in Sec. 6.1.4 attempts to treat heavy quark production consistently from threshold to the asymptotic region and the corresponding value of $\alpha_{s}$ is 0.118 , lower than the previous best fit value of MRSR2 $\left(\alpha_{s}=0.120\right)$.

To summarize, when one considers the systematic and theoretical uncertainties quoted on $\alpha_{s}\left(M_{Z}^{2}\right)$ values it is not clear that there is any significant difference between values of $\alpha_{s}$ extracted from lower energy DIS processes $\left(\alpha_{s} \sim 0.113\right)$ and those extracted from LEP data $\left(\alpha_{s} \sim 0.120\right)$, particularly in view of the updated CCFR $(97)$ result $\left(\alpha_{s} \sim 0.119\right)$. The values of $\alpha_{s}$ extracted from HERA data $\left(\alpha_{s} \sim\right.$ $0.120)$ are quite consistent with those extracted from the lower energy, fixed target DIS data and with the LEP data. However, a correct treatment of the correlations between systematic errors, of the charm threshold and of the need to include $\ln (1 / x)$ terms at low $x$ would all lead to a reduction of $\alpha_{s}$ compared to that extracted from a conventional NLO QCD fit made without these corrections. One awaits an analysis which includes all of these considerations before drawing final conclusions.

\subsection{Models for parton distribution functions}

One of the problems encountered in establishing whether DGLAP alone is sufficient at low $x$ and $Q^{2}$ is the flexibility allowed in the functional form chosen for the shape in $x$ of the PDFs at the starting scale $Q_{0}^{2}$. The actual forms used by the MRS and CTEQ groups have been discussed in Sec. 6.1.2 and we noted in Sec. 3.2 the constraints from counting rules as $x$ tends to 1 and from Regge theory at low $x$. Typically about 15 to 20 shape parameters are used in global fits to describe the nucleon PDFs. In this section we review very briefly some of the other methods and approaches that have been used to model or constrain the input distributions.

\subsubsection{Quantum statistics}

The idea that the Pauli exclusion principle could have an effect on the parton distributions and in particular force the sea to be flavour non-symmetric is almost as old 167 as the quark-parton model. There is also considerable support from experiment, as we have seen from the measured value of the Gottfried sum rule and the difference in the Drell Yan asymmetry measured in $p p$ and $p n$ scattering. Recently a number of authors have explored the use of functional forms based on Fermi-Dirac statistics for quarks and antiquarks and on Bose-Einstein statistics for gluons. So, for example, the quark distributions are of the form

$$
x q(x)=\frac{f(x)}{\exp \left(\frac{x-x_{q}}{\bar{x}}\right)+1}
$$

where $\bar{x}$ is a parameter playing the role of a universal temperature, $x_{q}$ is the thermodynamic potential of quark species $q$ and $f(x)$ is the weight function $A x^{\alpha}(1-x)^{\beta}$ 
allowing incorporation of the usual counting rule and Regge constraints if required.

Bucella, Bourrely, Soffer and coworkers 250 have developed a model for polarized parton distributions and have added phenomenological constraints, specifically the dominance of $u^{\uparrow}$ at large $x$ (from $F_{2}^{n} / F_{2}^{p}$ and $g_{1}^{p} / F_{1}^{p}$ ) and the approximate equality $u^{\downarrow}=d / 2$ (from QPM sum rules for $u^{\uparrow, \downarrow}$, the parton distribution functions for the quark spin parallel and antiparallel to that of the proton). They also split the quark distribution functions into two pieces: a 'gas' part corresponding to the valence quarks which is important at large $x$ and a common 'liquid' part to model the rise in the sea at low $x$. Ref.250 $d$ gives the most detailed comparison with data. The 8 or so model parameters are determined at the starting scale $Q_{0}^{2}=4 \mathrm{GeV}^{2}$ by fitting unpolarized CCFR(93) $x F_{3}$ data and $\operatorname{NMC}(92) F_{2}^{p}, F_{2}^{n}$ data. Standard NLO DGLAP evolution is then used to calculate the structure functions at other values of $Q^{2}$. The resulting PDFs give a reasonable description of $F_{2}^{p}, F_{2}^{n}$ at higher $Q^{2}$ (including the early data from HERA) and also the polarized structure functions $g_{1}^{p}$ and $g_{1}^{n}$. The model has been refined further 251 to produce various relationships between polarized and unpolarized structure functions. Another important outcome of these ideas is that the discrepancy in the Ellis-Jaffe sum rule 252 for $g_{1}^{p}$ may be linked to that in the Gottfried sum rule, but we will not pursue this further here.

A more formal extension of the use of statistical mechanics to parameterize the parton distributions is outlined in the paper by Mangano, Miele \& Migliore 253. Ideas from non-equilibrium statistical mechanics are used to derive modifications to the DGLAP evolution equations to take account of Pauli blocking for quarks and antiquarks and statistical effects in gluon emission. Finally Bhalerao 254 uses a phenomenological approach to consider how the statistical distributions are modified by the finite size of the nucleon. The effect of the finite volume is to soften the input parton distribution somewhat, which when coupled with standard DGLAP evolution allows the model to be fit to $F_{2}$ data quite successfully.

\subsubsection{The MIT bag model}

The MIT bag model 255 was developed to study the spectroscopy of hadronic states, but it also provides hadronic wavefunctions. There have been many attempts over the last 20 years to use bag model wavefunctions as a basis for calculating valence quark-parton distributions. In the bag model the quarks are taken to be massless fermions satisfying the free particle Dirac equation inside a static sphere of fixed radius. The spectrum of hadronic states is derived by applying the gluon field as a surface boundary condition on the confined quark fields. One of the major problems faced is that in a naive approach the bag model calculations do not give the correct support for the PDFs (i.e. they are non-zero for $x$ outside $[0,1])$. Procedures for overcoming this difficulty have been proposed by a number of authors and a fairly recent paper 256 emphasizes the importance of ensuring the correct support. Steffens and Thomas 257 have taken bag model parton distributions one step further by providing a calculation at next-to-leading order. While the quality of the description of the structure function data is not much changed, the 
reliability of the calculation is greatly increased. In leading order, to get a good fit the starting scale must be taken very low $\left(\mu^{2}=0.0676 \mathrm{GeV}^{2}\right)$ and the corresponding value of $\alpha_{s}$ is larger than 1 . In NLO the starting scale becomes $\mu^{2}=0.115 \mathrm{GeV}^{2}$ and $\alpha_{s}=0.77$. In both LO and NLO, apart from the starting scale, the bag radius and the scalar and vector di-quark masses are parameters to be determined from data. In fact they are fixed by comparison with the $\mathrm{MRSD}_{0}^{\prime}$ parameterization at $Q^{2}=$ $10 \mathrm{GeV}^{2}$. An alternative approach to the support problem has been investigated in a recent paper by Jasiak 258. His method also allows the parton distributions to be normalized correctly, thus addressing a common problem in bag model calculations. He compares his bag model calculations to the GRV94 parameterization and finds that it is necessary to use the Politzer scaling variable (which reduces the effects of target mass at low energies) to get approximate agreement at the starting scale.

\subsubsection{Lattice $Q C D$}

Much progress has been made in receptyears in the calculation of nucleon parton distribution functions from lattice QCD259. The present situation was summarized by Schierholz 260 at the recent DIS97 workshop. The lattice calculations are based

on the operator product expansion for the leading twist moments of the structure functions (Sec. 3), which gives

$$
\int_{0}^{1} d x x^{n-2} F_{2}\left(x, Q^{2}\right)=\sum_{a=u, d, g} C_{2, n}^{a}\left(\mu^{2} / Q^{2}, \alpha_{s}\left(\mu^{2}\right)\right) A_{n}^{a}(\mu)
$$

where $C_{2, n}$ are Wilson coefficients and $A_{n}$ are forward $\gamma^{*} p$ matrix elements of various local operators at scale $\mu$. In parton model language the matrix elements are related to the moments of the corresponding parton distributions, $A_{n}^{a}=q_{n}^{a}$. On the lattice the matrix elements are calculated from two and three point correlation functions, so far in the quenched approximation, ${ }^{\mathrm{t}}$ and in terms of bare lattice operators which are functions of the lattice spacing $d$. Calculations are done for a range of quark masses, typically $30-200 \mathrm{MeV} / \mathrm{c}^{2}$ so that the chiral limit may be taken reliably. To estimate the physical matrix elements (PDF moments) the operators must be renormalized at scale $\mu$ as they are divergent in $d$. The renormalization constants have been calculated perturbatively to one loop order. To date results have been obtained for moments up to $n=3$ as this is the maximum possible on a hypercubic lattice. A further constraint is that the required computing time increases rapidly with $n$. Table 8 shows results from ref.26 for moments of the unpolarized structure functions compared to those obtained from the CTEQ3M global fit.

The table shows that the lattice results for the lowest moments are systematically larger than those from the CTEQ global fit, particularly for the $u$ quark. Although not the subject of this review, it is worth noting that the same lattice calculations also give results for the moments of the polarized structure functions and these are in better agreement with measurements. The lattice group still have a large number

$\mathrm{t}$ Which means that the effects of internal quark loops are ignored. 
Table 8. Lattice QCD results 260 for moments of unpolarized parton distributions compared with those calculated from the CTEQ3M global fit. The lattice calculations were done in the quenched approximation and the figures in brackets give the error.

\begin{tabular}{lcc}
\hline Moment & $\begin{array}{c}\text { Lattice } \\
\mu^{2} \approx 5 \mathrm{GeV}^{2}\end{array}$ & $\begin{array}{c}\text { CTEQ3M } \\
\mu^{2}=4 \mathrm{GeV}^{2}\end{array}$ \\
\hline$\langle x\rangle^{u}$ & $0.410(34)$ & 0.284 \\
$\langle x\rangle^{d}$ & $0.180(16)$ & 0.102 \\
$\left\langle x^{2}\right\rangle^{u}$ & $0.108(16)$ & 0.083 \\
$\left\langle x^{2}\right\rangle^{d}$ & $0.036(8)$ & 0.025 \\
$\left\langle x^{3}\right\rangle^{u}$ & $0.020(10)$ & 0.032 \\
$\left\langle x^{3}\right\rangle^{d}$ & $0.000(6)$ & 0.008 \\
$\langle x\rangle^{g}$ & $0.53(23)$ & 0.441 \\
\hline
\end{tabular}

of systematic effects to study, for example lattice spacing, improved actions and the effect of the quenched approximation. However they do not think that these will change the pattern of the above results significantly. One possible explanation is that higher twist effects in the data are responsible for the difference. This is an area that is beginning to receive attention again for a number of reasons, but it will take a lot more study, both from the lattice and phenomenological sides before this explanation is established.

In principle the idea of using lattice QCD to give the parton distribution functions at the starting scale is a very attractive as one would then be able to compare data at all $Q^{2}$ with a complete QCD calculation. However, leaving aside the present discrepancy in the unpolarized moments, it will be a long time before lattice technology is able to provide calculations of other than the lowest order moments. The challenge of how to use the incomplete information provided by the low order moments from the lattice has been taken up by Mankiewicz \& Weigl 261. Their idea is to use the lattice moments to give the large $x$ behaviour of the parton distributions and phenomenological or Regge ideas to fix the small $x$ behaviour. Technically they do this by using the Ioffe-time distributions 262, effectively transforming the parton distributions to coordinate space. To be more specific the parton distribution function $q\left(x, \mu^{2}\right)$ and the Ioffe distribution $Q\left(z, \mu^{2}\right)$ are related by

$$
\begin{aligned}
Q\left(z, \mu^{2}\right) & =\int_{0}^{1} d u q\left(u, \mu^{2}\right) \sin u z \\
q\left(x, \mu^{2}\right) & =\frac{2}{\pi} \int_{0}^{\infty} d z \quad Q\left(z, \mu^{2}\right) \sin u z
\end{aligned}
$$

where $z$ is the Ioffe-time variable, which is a Lorentz invariant measure of the longitudinal distance along the light cone between the quark fields. $Q\left(z, \mu^{2}\right)$ is the gauge invariant correlation function of two quark fields on the light cone. The advantage of using $Q\left(z, \mu^{2}\right)$ is that it allows the separation, in a very clear way, of the two different regimes (large and small $x$ ) which determine the behaviour of the PDFs. Behaviour at large $z$ corresponds to small $x$ and this has to be determined phenomenologically at present. One finds that if $q(x) \sim x^{-(1+\alpha)}$ then $Q(z) \sim z^{\alpha}$ 
and vice-versa. The authors have used CTEQ3, MRSA and GRV94 to evaluate the singlet $Q(z)$ distributions for the $u$ and $d$ quarks at the scale $\mu^{2}=4 \mathrm{GeV}^{2}$. In all cases they find that the large $z$ behaviour is approximately constant or slowly rising, indicating that $\alpha$ is either 0 or a small positive number. The results also indicate that at large $z>10$ (small $x$ and corresponding to linear distances of order $2 \mathrm{fm}$ ) quark-quark correlations at distances larger than the electromagnetic size of the nucleon are important. The behaviour at small values of $z$ (large $x$ ) is almost linear and in principle well determined by the low order parton distribution moments from lattice QCD. The two different behaviours have to be matched smoothly at a transition region around $z=5$. The scheme that Mankiewicz and Weigl use is a power series interpolation in $z$ with the parameters determined from the lattice moments at small $z$, and from the CTEQ NLO fit and the nucleon electromagnetic radius at large $z$. The resulting singlet distribution for the $u$ quark is larger than that from global fits, which is a reflection of the larger lattice moments. A similar approach for the non-singlet valence quark distributions gives results in better agreement with global fits, which indicates that perhaps the contribution of the $q \bar{q}$ sea at small $x$ is not properly understood yet.

\subsubsection{Other approaches}

It is clear from the discussion above that a different approach is needed to calculate the small $x$ behaviour of the structure functions. Recently there has been a revival of interest in non-perturbative methods that use other than quark degrees of freedom 263. The ideas go back to those of Skyrme and more recently the instanton 264, which are based on classical solutions of Euclidean QCD field equations. QCD has chiral symmetry which is spontaneously broken giving rise to the almost massless pion as the corresponding Goldstone boson. Diakonov, Petrov and coworkers 265 have used these ideas to calculate the quark momentum distributions in the large- $N_{c}$ limit and at a low scale. The basis of their model is that in the large$N_{c}$ limit QCD is equivalent to an effective meson theory with baryons as solitonic excitations. The effective theory is expressed in the form of a chiral lagrangian for the pion field. There are various parameters in the model, such as the constituent quark mass which is taken to be $350 \mathrm{MeV} / \mathrm{c}^{2}$ and a UV cutoff (technically a PauliVillars regularization of $600 \mathrm{MeV}$ is used in the numerical calculations). The latter parameter also sets the scale at which the parton distributions are calculated. The calculated isosinglet singlet unpolarized distributions are compared with the NLO GRV94 parameterization and the isovector polarized distributions with the corresponding parameterizations from ref.266. In both cases the agreement between the calculated and the phenomenological distributions (which are taken as a good indication of the behaviour of the data) is quite good in shape but the normalizations do not quite agree. Fig. 47 shows the comparison for the isosinglet unpolarized distribution.

From the figure it can be seen that the model lies above the 'data' in the isosinglet case. For the isovector it is the other way around. Diakonov et al argue that this 


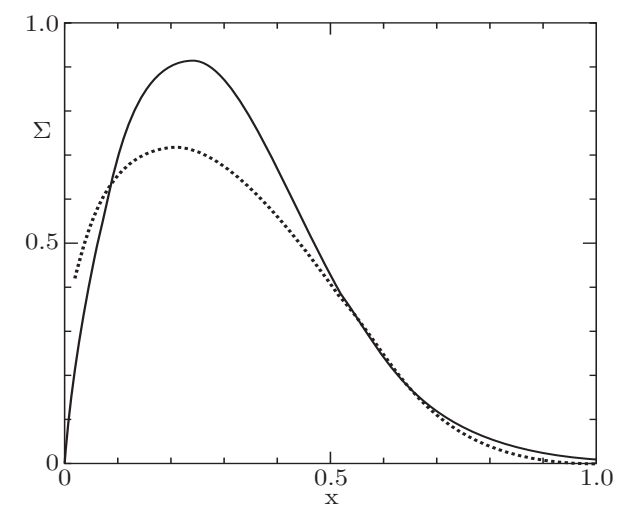

Fig. 47. The isosinglet unpolarized distribution $\Sigma=\frac{1}{2} x[u+d+\bar{u}+\bar{d}]$ from the large $N_{c}$ chiral model calculation of Diakonov et al (line) compared to that from GRV94 (points).

is very encouraging as the calculations can be improved and that they already give a lot of support to the GRV approach of a low starting scale with 'valence-like' input distributions. A somewhat similar approach based gn the Nambu-Jona-Lasino model is discussed by Gamberg, Reinhardt and Weigl 267.

Another recent calculation that gives support to the GRV assumption of an intrinsic 'valence like' gluon momentum distribution is that of Hoyer and Roy 268. It is based on an analysis of the long time-scale structure of the nucleon using a Fock state decomposition of the nucleon wave-function proposed some years ago 269. The basic idea is that the partons in the nucleon Fock states $(|q q q\rangle,|q q q g\rangle,|q q q q \bar{q}\rangle, \ldots)$ have similar velocities in order to stay together over long times. This then gives a probability for an $n$-parton state proportional to $\left(m_{N}^{2}-\sum_{1}^{n} \frac{m_{\perp i}^{2}}{x_{i}}\right)^{-2}$, where $m_{\perp i}^{2}=m_{i}^{2}+k_{\perp i}^{2}$ is the squared transverse mass of parton $i$. This leads to the probability distribution of a given Fock state being peaked at $x_{i}=m_{\perp i} / \sum_{i}^{n} m_{\perp i}$. For the 3 quark plus multi-gluon states, assuming a common transverse mass $m_{\perp i} \approx$ $0.3-0.4 \mathrm{GeV}$, the probability of the $n$-gluon state is found to be proportional to $1 / n^{4}$. This then gives $\overline{x_{q}}=1 / 4, \overline{x_{g}}=1 / 6$ for the mean fractional momenta carried by the quarks and gluons and $\epsilon_{q}=3 / 4, \epsilon_{g}=1 / 4$ for the fractions of the nucleon momentum at the starting scale, where both quark and gluon states have a valencelike shape.

To summarize this brief survey of non-perturbative models for the PDF input functions, we have seen that there are some very promising ideas but that much more work needs to be done before one could use such results directly in a global fit. A more likely scenario is to use some of the non-perturbative results as constraints on the input functional forms and parameters - as Mankiewicz and Weigl have done with the structure function moments from lattice calculations. It is also interesting to note that a number of the model approaches do appear to give support to the 
GRV valence-like input forms.

\section{7. pQCD at low $x$}

With the advent of data from the HERA collider we are into a new phase of testing the applicability of pQCD. This time we are interested in testing its predictions at very low $x$ where we may be moving out of the region where the conventional approximations (LLA and NLLA) as embodied in the DGLAP equations are applicable. We outline the main theoretical approaches to the low $x$ region in Sec. 7.1 and we discuss the current phenomenology of each of these approaches in the subsequent sections.

\subsection{Theoretical approaches to low $x$ physics}

\subsubsection{Low $x$ solutions of the DGLAP equations}

Consider Eqs. 64- 67 for the (LO) splitting functions as $z=x / y \rightarrow 0$.

$$
P_{q q} \rightarrow \frac{4}{3}, P_{q g} \rightarrow \frac{1}{2}, P_{g q} \rightarrow \frac{4}{3} \frac{1}{z}, P_{g g} \rightarrow 6 \frac{1}{z}
$$

We see that the gluon splitting functions are singular as $z \rightarrow 0$ (and this result remains true for higher orders). Thus the gluon distribution will become steep as $x \rightarrow 0$, and its contribution to the evolution of the quark distribution will become dominant, so that the quark singlet distributions, and hence the structure function $F_{2}$, will also become steep as $x \rightarrow 0$.

Quantitatively,

$$
\frac{d g\left(x, Q^{2}\right)}{d \ln Q^{2}} \simeq \frac{\alpha_{s}\left(Q^{2}\right)}{2 \pi} \int_{x}^{1} \frac{d y}{y} \frac{6}{z} g\left(y, Q^{2}\right)
$$

may be solved subject to the nature of the boundary function $x g\left(x, Q_{0}^{2}\right)$. There are two possibilities, depending on whether this input function is singular or nonsingular. Formally, the distinction is made by considering whether the singularities of the operator product expansion matrix elements lie to the left or the right of those of the anomalous dimensions. In practice, this means that if the input function is flatter(steeper) than $x g\left(x, Q_{0}^{2}\right) \sim x^{-0.25}$ it is considered non-singular(singlerp).

The non-singular solution was given (in the DLLA) by de Rujula et al 270

$$
x g\left(x, Q^{2}\right) \simeq \exp \left(2\left[\xi\left(Q_{0}^{2}, Q^{2}\right) \ln \frac{1}{x}\right]^{\frac{1}{2}}\right)
$$

where

$$
\xi\left(Q_{0}^{2}, Q^{2}\right)=\int_{Q_{0}^{2}}^{Q^{2}} \frac{d q^{2}}{q^{2}} \frac{3 \alpha_{s}\left(Q^{2}\right)}{\pi}
$$

Given a long enough evolution length from $Q_{0}^{2}$ to $Q^{2}$, this will generate a steeply rising gluon distribution at small $x$, starting from the flattish behaviour (or even a valence-like shape 220) of $x g\left(x, Q^{2}\right)$ at $Q^{2}=Q_{0}^{2}$. 
Specifically, the gluon distribution rises faster than any power of $\ln (1 / x)$, but slower than any power of $x$. However, over the limited $x, Q^{2}$ range of HERA data it may mimic a power behaviour, $x g\left(x, Q^{2}\right) \sim x^{-\lambda_{g}}$, with

$$
\lambda_{g}=\left(\frac{12}{\beta_{0}} \frac{\ln \left(t / t_{0}\right)}{\ln (1 / x)}\right)^{\frac{1}{2}}
$$

where $t=\ln \left(Q^{2} / \Lambda^{2}\right), t_{0}=\ln \left(Q_{0}^{2} / \Lambda^{2}\right)$. The steep behaviour of the gluon will generate a seep behaviour of $F_{2}$ at small $x, F_{2} \sim x^{-\lambda_{S}}$, where the DGLAP evolution kernels give271

$$
\lambda_{S} \approx\left(\frac{12}{\beta_{0}} \frac{\ln \left(t / t_{0}\right)}{\ln (1 / x)}\right)^{\frac{1}{2}}-\frac{a}{\ln (1 / x)}
$$

where $a$ depends on the input shape of the gluon at $t_{0},(a=0.75$ for a flat input, $a=1.25$ for a valence-like input). In Fig. 48 we illustrate the behaviour of this equation by showing $\lambda_{S}=d \ln F_{2} / d \ln (1 / x)$ as a function of $Q^{2}$ for two different $x$ values.

This steep behaviour of $F_{2}$ at small $x$ contrasts with the Regge expectation of a flattish behaviour of $F_{2}$ coming from the exchange of the soft Pomeron (see Sec. 3.2). However, there is not necessarily any contradiction between these two predictions, since it is not clear over what range of $Q^{2}$ the Regge prediction should be applicable. If we assume it is appropriate for very low $Q^{2}$ values, then pQCD will generate a steep shape for higher $Q^{2}$ by its evolution. This is the usual explanation of conventiopal DGLAP evolution for the steep shape of $F_{2}$ observed at low $x$ in HERA data 219.220. The current phenomenology of this explanation is explored in Sec. 7.2 .

This approach suggests that we might extend our concept of the Pomeron. The Regge prediction was based on the exchange of the soft Pomeron in the t-channel. If we continue this idea into the higher $Q^{2}$ realm it implies that the Pomeron can be identified with the strongly ordered gluon ladder of Fig. 11. Retracing the argument that a flattish behaviour of $F_{2}$ as $x \rightarrow 0$ derives from a flattish behaviour of the $V^{*} N$ total cross-section as $s\left(V^{*} N\right)\left(=W^{2}\right)$ increases, we see that a steeply rising behaviour of $F_{2}$ as $x \rightarrow 0$ would imply a steeply rising $V^{*} N$ total cross-section, and the data for larger $Q^{2}$ do indeed exhibit this behaviour, see Fig. 71 . Hence the corresponding 'QCD' Pomeron is hard, i.e. it has an intercept $\alpha_{P}$ significantly in excess of unity.

The singular solution to Eq. 160 has been given by Yndurain et al24.445272. If a steep $\lambda$ value is input then it remains stable underion, overriding the prediction of de Rujula et al 270 such that one has $185,219,158$

$$
F_{2} \sim \ln Q^{2} x^{-\lambda}
$$

with a fixed value of $\lambda$ equal to the input value, for all $Q^{2}$. Whereas such a solution implies an unconventional hard Pomeron right from the start, it may be preferred theoretically because such solutions are stable against the inclusion of higher order diagrams, whereas the solutions with flattish (or valence-like) input gluons are quite 


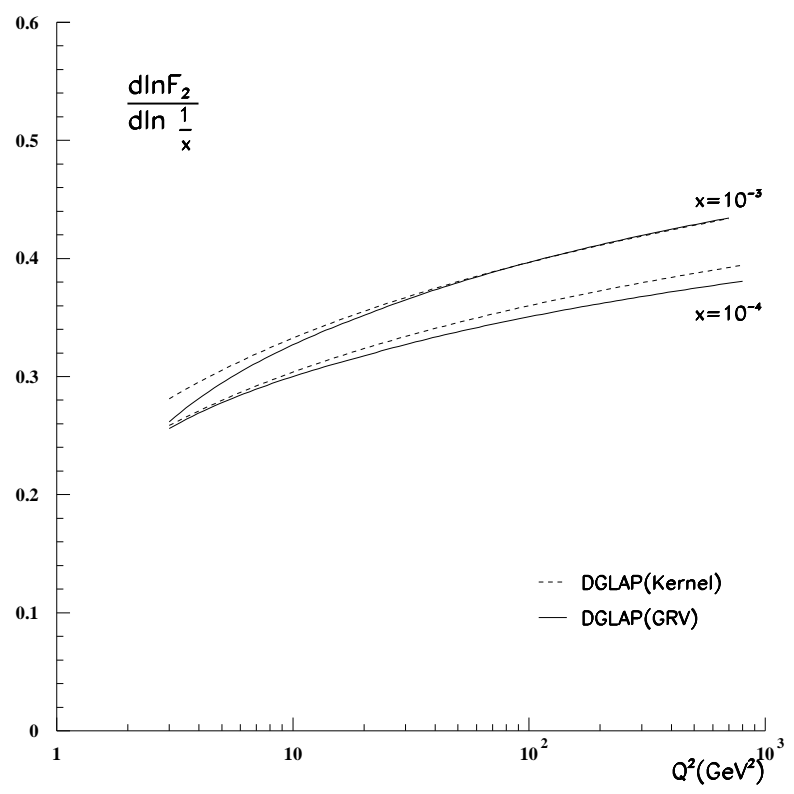

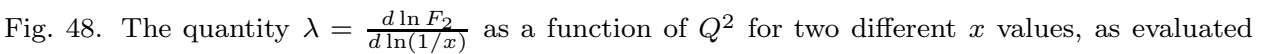
from the DGLAP kernels 271 and from the parametrization of GRV

strongly sensitive to the inclusion of NNLO and NNNLO terms 185. The current phenomenology of this solution is explored further in Sec. 7.2

\subsubsection{The BFKL equation}

Although it is now evident that it is possible to fit all data with $Q^{2} \gtrsim 1.5 \mathrm{GeV}^{2}$ conventionally, there have been criticisms of this explanation. Firstly, because it is necessary to begin the $Q^{2}$ evolution at $Q^{2} \simeq 1.0 \mathrm{GeV}^{2}$, or lower, and one may doubt whether perturbative calculations are reliable for such low $Q^{2}$ values, since $\alpha_{s}$ is correspondingly large $\left(\alpha_{s} \gtrsim 0.4\right)$. Secondly, in the usual LLA approach we are summing terms which are leading in $\ln (1 / x)$ only when they are accompanied by leading $\ln Q^{2}$. One might legitimately ask if, in the low $x$ region, it would not also be appropriate to sum diagrams which are leading in $\ln (1 / x)$ independent of $\ln Q^{2}$. This is what is done by the BFKL equation 187. This involves considering the evolution of a gluon distribution which is not integrated over $k_{T}$, since breaking the association to leading $\ln Q^{2}$ implies that the gluon ladder need not be ordered in $k_{T}$. The unintegrated gluon distribution $f\left(x, k_{T}^{2}\right)$ relates to the more familiar gluon distribution as follows

$$
x g\left(x, Q^{2}\right)=\int_{0}^{Q^{2}} \frac{d k_{T}^{2}}{k_{T}^{2}} f\left(x, k_{T}^{2}\right)
$$


The BFKL equation may then be written

$$
\frac{d f\left(x, k_{T}^{2}\right)}{d \ln (1 / x)}=\int d k_{T}^{\prime 2} K\left(k_{T}^{2}, k_{T}^{\prime 2}\right) f\left(x, k_{T}^{\prime 2}\right)=K \otimes f=\lambda f
$$

which describes the evolution in $\ln (1 / x)$ of the unintegrated gluon density.

The solution of this equation is controlled by the largest eigenvalue $\lambda$ of the kernel $K$ u. To leading order in $\ln (1 / x)$ ), and fixed $\alpha_{s}$, we obtain the very steep power law behaviour

$$
x g\left(x, Q^{2}\right) \sim f\left(Q^{2}\right) x^{-\lambda_{L}}
$$

where

$$
\lambda_{L}=\frac{3 \alpha_{s}}{\pi} 4 \ln 2 \simeq 0.5
$$

(for $\alpha_{s} \simeq 0.25$, as appropriate at $Q^{2} \sim 4 \mathrm{GeV}^{2}$ ). This would lead to very steeply rising $V^{*} N$ cross-sections, corresponding to a hard Pomeron of intercept $\alpha_{P}=1.5$. This hard Pomeron has been termed the BFKL Pomeron.

This power law behaviour of the BFKL solution could explain data which is already steeply rising at moderate $Q^{2}$, without need of a long evolution length from $Q_{0}^{2}$ to $Q^{2}$, and hence without need to use perturbative QCD at very low $Q^{2}$. The power $\lambda_{L} \simeq 0.5$ is too steep to fit current data, however the simple derivation just sketched should be improved in various ways. One must solve the full equation with an appropriate boundary conditions rather than consider just the leading eigenvalue. Then one must consider incorporating the running of $\alpha_{s}$ with $Q^{2}$ (or more appropriately $k_{T}^{2}$ ) and one must consider the upper(UV) and lower(IR) cut-offs on the $k_{T}^{2}$ integration in Eq. 167 and finally one must bear in mind that the kernel of the equation has only been completely calculated to leading order in $\ln (1 / x)(L L(1 / x))$. All component parts for next-to-leading order $(N L L(1 / x))$ calculations are now complete, but it is not yet clear how to implement them274, 279,276. The current phenomenology of the BFKL equation is discussed in Sec. 7.3.

\subsubsection{Unifying the DGLAP and BFKL equations}

Whereas the conventional DGLAP equations deal with $Q^{2}$ evolution and may be inadequate at low $x$, the BFKL equation deals with $1 / x$ evolution and may be inadequate at high $Q^{2}$. Fig. 49 gives a diagram of the applicability of various evolution equations across the $x, Q^{2}$ plane. One would like to have an approach which can be used throughout the kinematic plane. One possible way is to attempt to incorporate extra terms in $\ln (1 / x)$ into the DGLAP equations ('Unconventional' DGLAP). This amounts to recalculating the splitting functions and coefficient functions to 'resum' all $\ln (1 / x)$ contributions. If we rewrite Eq. 62 for small $x$, where the gluon is dominant, as follows

$$
\begin{gathered}
\frac{d g\left(x, Q^{2}\right)}{d \ln Q^{2}}=\int_{x}^{1} \frac{d y}{y} P_{g g}\left(\frac{x}{y}, \alpha_{s}\right) g\left(y, Q^{2}\right) \\
P\left(x, \alpha_{s}\right)=P^{0}(x) \alpha_{s}\left(Q^{2}\right)+P^{1}(x) \alpha_{s}^{2}\left(Q^{2}\right)+\ldots
\end{gathered}
$$

\footnotetext{
$\mathrm{u}$ For the form of the BFKL kernel and details of the techniques for solution see Kwiecinski 273 .
} 


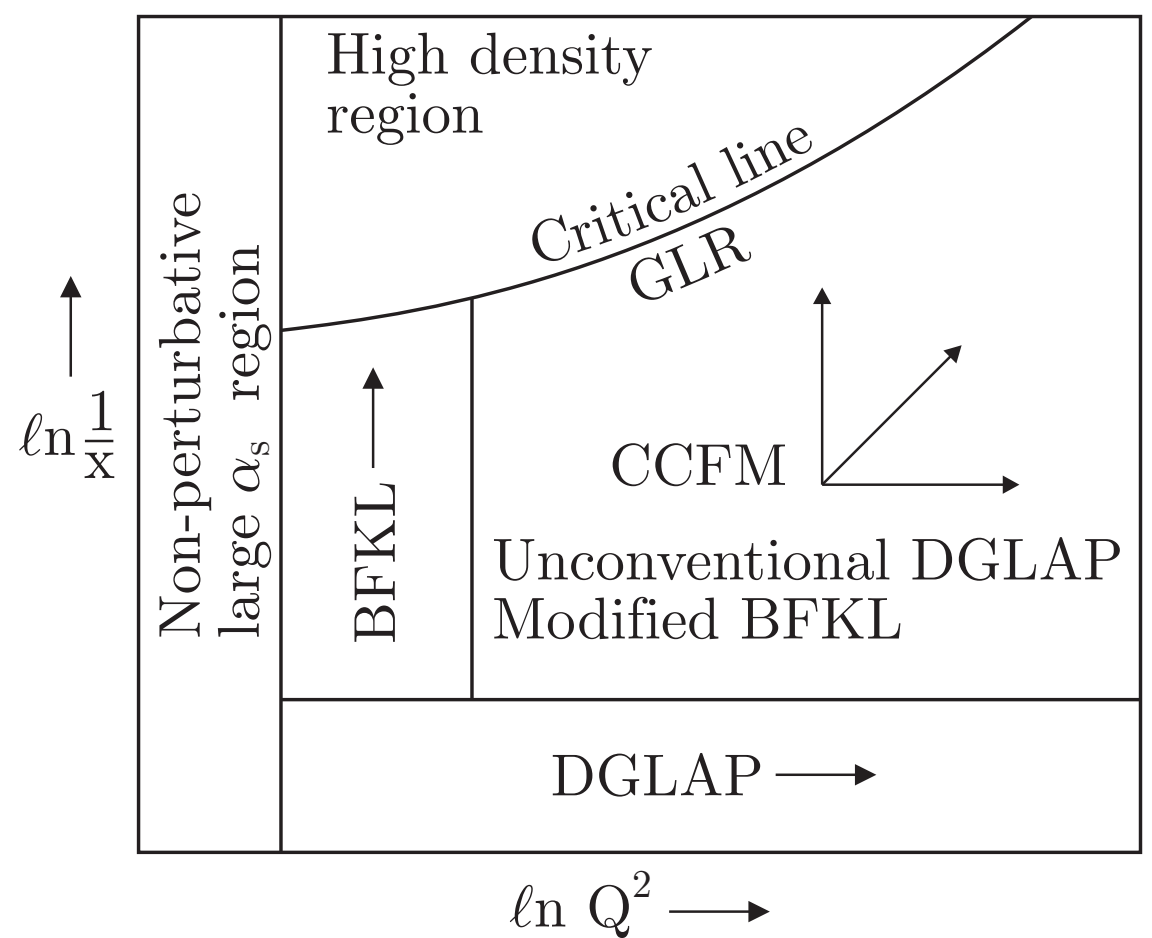

Fig. 49. Schematic representation of the applicability of various evolution equations across the $x, Q^{2}$ plane

in order to bring out the dependence on $x$ and $Q^{2}$, then at small $x$ we have

$$
P^{n}(x)=\frac{1}{x}\left[a_{n} \ln ^{n}\left(\frac{1}{x}\right)+b_{n} \ln ^{n-1}\left(\frac{1}{x}\right)+\ldots\right]
$$

so that in general

$$
x P\left(x, \alpha_{s}\right)=\Sigma_{n=1}^{\infty} \Sigma_{m=1}^{n} A_{n m} \alpha_{s}^{n}\left(Q^{2}\right) \ln ^{m-1}\left(\frac{1}{x}\right)
$$

and the coefficient functions may be expanded similarly. It is convenient to write this in terms the anomalous dimensions, as follows

$$
\gamma^{N}\left(\alpha_{s}\right)=\Sigma_{n=1}^{\infty} \Sigma_{m-\infty}^{n} A_{n m} \alpha_{s}^{n} N^{-m}
$$

where the sum over $m$ extends to negative values to represent the contribution of the terms which are non-singular as $x \rightarrow 0$, and $N$ is the moment index (see Sec. 3.3.4 . Ideally we should sum the whole of $n, m$ space, but in practice we make different approximations as to which terms are most important. Conventionally we consider terms which are leading in $\ln Q^{2}$ so we sum Eq. 174 over $m$ for $n=1$ in the LLA, and for $n=2$ as well in the NLLA. However one could re-order the sum to first sum over terms with a leading $\ln (1 / x)(L L(1 / x))$. These are the terms for which $n=m$ and this approximation gives the BFKL equation at $L L(1 / x)$. Terms with $m=n-1$ give the $N L L(1 / x)$ approximation. The common point $n=m=1$ 


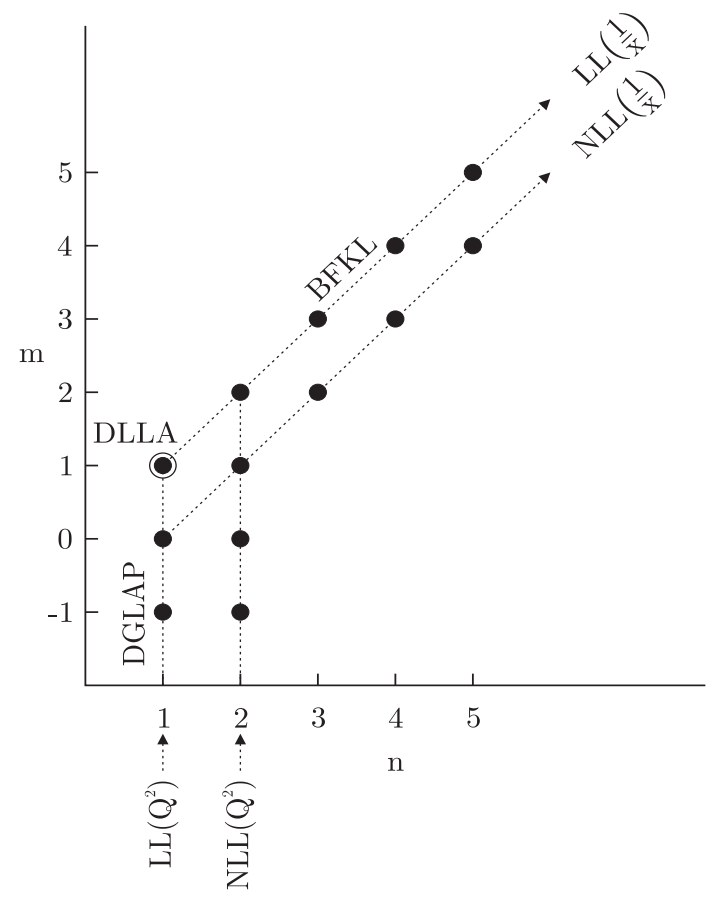

Fig. 50. The $n, m$ plane with the BFKL and DGLAP summations indicated

represents the DLLA. The $n, m$ plane and these differing summations are illustrated in Fig. 50 .

We are defining different expansion schemes to sum up the contributions of different terms to the evolution of the parton distributions, and eventually to the $\gamma^{*}-$ parton cross-section. The conventional large $x$ scheme, where $\ln Q^{2}$ is considered leading, sums up all logs of the form

$$
\alpha_{s}^{p}\left(\ln Q^{2}\right)^{q}\left(\ln \frac{1}{x}\right)^{r}
$$

for which $p=q \geq r \geq 0$, at LO. At NLO terms for which $p=q+1 \geq r \geq 0$ are also summed. In the small $x$ scheme, where $\ln (1 / x)$ is considered leading, terms for which $p=r \geq q \geq 1$ are summed at $L L(1 / x)$, and terms for which $p=r+1 \geq q \geq 1$ are also summed at $N L L(1 / x)$. This small $x$ scheme can be used to supplement the conventional splitting functions (and coefficient functions) in order to include $\ln (1 / x)$ higher order terms appropriately at small $x$ within the framework of the DGLAP equations. The current phenomenology of this approach is discussed in Sec. 7.4

Another way to approach the need for a solution applicable across the whole kinematic plane is to develop alternative evolution equations which sum more general classes of diagrams. The CCFM equation 277278 is the best established of such equations. It is based on the idea of coherent gluon radiation, which leads to angular ordering of gluon emissions in the gluon ladder such that $\theta_{i}>\theta_{i-1}$, where $\theta_{i}$ is the 
angle that the $i$ th gluon makes to the original direction (see Fig. 11). Outside this angular region there is destructive interference such that multi-gluon contributions vanish to leading order. Angular ordering implies ordering in the transverse momenta $k_{T}$ divided by the energies $E$ of the gluons on the rungs of the gluon ladder. An additional scale is necessary to specify the maximum angle of gluon emission. This extra scale can be taken to be the scale $Q$ of the probe, such that we deal with a scale dependent unintegrated gluon density $f\left(x, k_{T}^{2}, Q^{2}\right)$. The integral equation for $f\left(x, k_{T}^{2}, Q^{2}\right)$ can be approximated by the BFKL equation at small $x$, where $f$ becomes independent of $Q^{2}$ and ordering in $k_{T} / E$ does not imply $k_{T}$ ordering. However, at moderate $x$, ordering in $k_{T}$ is implied and one recovers the DGLAP equation for the integrated gluon distribution $g\left(x, Q^{2}\right)$. The CCFM equation takes into account some of the effects of the next-to-leading order $\ln (1 / x)$ terms which have yet to be implemented in the BFKL equation 279.280281.

Li 282, 283 has taken an alternative approach to the unification of the DGLAP and BFKL equations. He proposes a modified BFKL equation which contains an intrinsic $Q^{2}$ dependence which does not come from a $\ln Q^{2}$ summation but from consideration of the phase space boundary for radiative corrections.

Kwiecinski, Martin and Stasto 284 have developed a different modified BFKL equation which incorporates both $\ln (1 / x)$ and $\ln Q^{2}$ resummation, such that the equation maybe used over all $x$ and $Q^{2} \gtrsim 1 \mathrm{GeV}^{2}$. A major uncertainty in the solution of the BFKL equation comes from the treatment of the infra-red region. The modified equation improves on this by only considering $f\left(x, k_{T}^{2}\right)$ in the perturbative domain.

The CCFM equation and these modified BFKL equations may be preferable to the approach which incorporates $\ln (1 / x)$ resummation within DGLAP since they all deal with an unintegrated gluon density and hence preserve more of the physics of the non- $k_{T}$ ordered gluon ladder. The current phenomenology of the CCFM equation and of modified BFKL equations is discussed in Sec. 7.5

\subsubsection{Non-linear effects and higher twist at low $x$}

Two further, related questions occur when considering the implications of a steeply rising gluon density at small $x$. The first is that a steeply increasing $V^{*} N$ cross-section would eventually violate the Froissart (unitarity) bound. This bound may, of course, not be applicable in the case of particles off-mass shell285, but one can view the problem from a slightly different perspective which needs consideration independent of such a violation. As $x \rightarrow 0$ the gluon density is becoming very large so that the possibility of gluon annihilation, or recombination, may compete with the usual evolution. Another way of expressing this is to say that the gluons shadow, or screen, each other from the virtual-boson probe. A possible multi-ladder diagram representing such a gluon-gluon interaction is given in Fig. 51 .

An estimate of the size of gluon density necessary for shadowing to become important may be given as follows. A measurement at the scale $Q^{2}$ probes a gluon of transverse size $\sim 1 / Q$, longitudinal size $\sim 1 /(x P)$. The number of gluons 


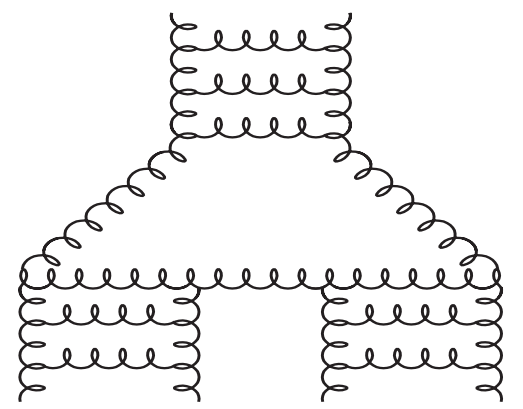

Fig. 51. An example of a multi-ladder diagram representing gluon-gluon interaction

per unit rapidity $\ln (1 / x)$ which can interact with the probe is $x g\left(x, Q^{2}\right)$, so the transverse area which they occupy is $x g\left(x, Q^{2}\right) \pi / Q^{2}$. When this is comparable to $\pi R^{2}$, the transverse area of the nucleon, we should expect to get parton shadowing 286, i.e. when $x g\left(x, Q^{2}\right) \gtrsim R^{2} Q^{2}$. For $R \sim 1 \mathrm{fm}$ and $Q^{2} \sim 10 \mathrm{GeV}^{2}$ we obtain $x g\left(x, Q^{2}\right) \gtrsim 250$. However there is a large uncertainty on this estimate, a more realistic value for the area occupied by the gluons may be $x g\left(x, Q^{2}\right) 4 \pi / Q^{2}$, which would already give shadowing for $x g\left(x, Q^{2}\right) \gtrsim 60$, at $Q^{2} \sim 10 \mathrm{GeV}^{2}$. Such values of the gluon momentum distribution have not yet been reached even for the lowest $x$ data at HERA. However the relevant size for $R$ may not be the nucleon radius, but the radius of a constituent quark $(\sim 0.4 \mathrm{fm})$ if the gluon ladders of Fig. 51 couple to the same parton within the nucleon. In this case shadowing may be expected in HERA data. Such a scenario is called 'hot-spot' shadowing 287, see Fig. 22.

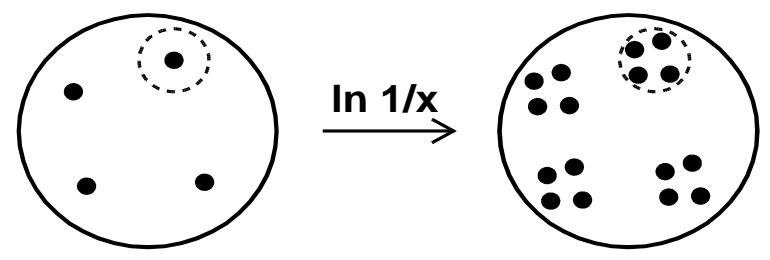

Fig. 52. Schematic diagram showing the increase in local parton density due to evolution in $x$, leading to hotspot regions in the proton.

In considering shadowing we are moving towards a new region in the $x, Q^{2}$ plane where the parton density is high (see Fig. 49) and the usual methods of perturbative QCD cannot be applied despite the fact that $\alpha_{s}$ is still quite small. We need quantum statistical methods which are in process of development. It is useful to define a parameter, $\omega\left(x, Q^{2}\right)=\frac{\alpha_{s}}{Q^{2}} \rho$, where $\rho=\frac{x g\left(x, Q^{2}\right)}{\pi R^{2}}$ is the gluon density in the transverse plane. Since $\alpha_{s} / Q^{2}$ gives the cross-section for gluon absorption by a parton in the hadron, $\omega$ represents the probability of gluon recombination (or 
shadowing). When $\omega \ll \alpha_{s}$ shadowing is negligible and we may use linear evolution equations such as BFKL or DGLAP. When $\omega \gg \alpha_{s} \lesssim 1$ we have reached the high density region and we approach the unitarity limit. When $\omega \simeq \alpha_{s}$ shadowing is significant but parton densities are not overwhelmingly large. In Fig. 49 we indicate a 'critical line' separating this region from the very high density region. (Note that this figure is only schematic and the relative positions of many of the lines is still in debate, for example Mueller 288 contends that the non-perturbative region extends to higher $Q^{2}$ as $x$ decreases such that it may subsume the high density region (see also ref.289) and Levin 276 contends that the BFKL region may be completely hidden in the shadowing region).

To the right of this critical line a first attempt to account for shadowing was developed in which one adds a non-linear term to the usual evolution equations. The probability of evolution, or parton splitting is $\propto \alpha_{s} \rho$, and the probability of recombination is $\propto \alpha_{s}^{2} \rho^{2} / Q^{2}$, so that the evolution of the gluon distribution is given by

$$
\frac{d^{2} x g\left(x, Q^{2}\right)}{d \ln Q^{2} d \ln (1 / x)}=\frac{3 \alpha_{s}}{\pi} x g\left(x, Q^{2}\right)-\frac{\alpha_{s}^{2} \gamma}{Q^{2} R^{2}}\left[x g\left(x, Q^{2}\right)\right]^{2}
$$

where the proportionality factor $\gamma=81 / 16$ is calculated from Regge theory as the triple Pomeron (or triplegluon ladder) vertex (see Fig. 51). This equation is known as the GLR equation 290. When $x g\left(x, Q^{2}\right) \sim \pi Q^{2} R^{2} / \alpha_{s}\left(Q^{2}\right)$ the non-linear term cancels the linear term and the evolution of $x g\left(x, Q^{2}\right)$ stops. This is known as saturation. Correspondingly the steep rise in the gluon distribution would flatten off and the $V^{*} N$ cross-section would also flatten off, hence there would be no violation of the unitarity bound. However we have now moved to the left of the critical line and the GLR equation cannot be trusted.

There have been some phenomenological attempts to consider shadowing corrections to both the DGLAP and the BFKL equations 159291292 using this simple picture. Gotsman et al 292 have suggested that such corrections are responsible for the reduction of the BFKL slope $\lambda_{L}$ to match the observed values. However the GLR equation has been criticized as inadequate even in its supposed region of validity. Zhu et al293 have pointed out that the GLR equation does not conserve the momentum of the gluons and have proposed a modified GLR equation. Bartels 294 has pointed out that there may be ladder-ladder interactions before the recombination and furthermore, considering the recombination of just two gluon ladders may not go far enough, multi-ladder diagrams may also be important. An extension of the GLR equation to account for multi-ladder correlations was developed by Laenen and Levin 295 and, more recently, the Geiger-Mueller 296 approach to shadowing has been developed by Ayala, Gay-Ducati and Levin297 such that results should be applicable closer to the critical line. This work has suggested that shadowing effects become significant in the low $x$ region before it is necessary to take into account large $\alpha_{s} \ln (1 / x)$ terms (see also ref.298).

The interpretation of shadowing in terms of the Operator Product Expansion of operators of different twists is not obvious. A more rigorous evaluation of higher twist effects at small $x$ is undoubtedly necessary. We expect twist-4 gluon operators 
to be most important at small $x 299$. The relevant operators are the 2-gluon and 4gluon operators and these may mix via a $4 \rightarrow 2$ gluon transition vertex. Bartels 300 has recently made a phenomenological study of such higher twist effects at low $Q^{2}$. He uses the fact that diffractive vector meson production has been established as a twist-4 effect to put a lower limit on the twist-4 contribution to DIS of a few percent at $Q^{2} \sim 10 \mathrm{GeV}^{2}, x \sim 10^{-3}$. The resulting small $x$ behaviour of the twist- 4 contribution to $F_{2}$ comes out as negative and its expected $1 / Q^{2}$ fall off is compensated by a strong dependence on $Q^{2}$ of the twist- 4 scaling violations ${ }^{\mathrm{v}}$ such that twist-4 effects may remain important to unexpectedly large $Q^{2}$.

A new picture of the physics of the BFKL equation has been developed by considering the interaction of chains of colour dipoles in impact parameter space 301 . This picture may also enable us to study saturation and unitarity effects, which occur when the dipole density grows, in a theoretically consistent manner 302. There have also been studies of the high density region in which attempts are made to formulate a Lagrangian for high density QCD 03 . For example, one may replace the complex QCD interaction between partons by the interaction of a parton with momentum fraction $x$ with a classical field created by all partons with momentum fraction larger than $x$. The DGLAP and BFKL equations are recovered at the correct low density limits. There are also formal studies of this region which build on the old idea of Reggeon Calculus, but using reggeized gluons (i.e. BFKL Pomeron) as the basic object in an effective field theory 04 .

Higher twist effects, shadowing, parton saturation and the approach to the critical line are also of relevance to the second question which arises when considering a steeply rising gluon density. Namely, whereas the $V^{*} N$ cross-section does rise steeply with $W^{2}$ (for small $x$ ) the real photon nucleon cross-section does not. We need to understand this transition from hard to soft physics, but we will require non-perturbative techniques since perturbative $Q C D$ cannot be used when $Q^{2}$ is small and $\alpha_{s}$ is correspondingly large. Bartels 300 has suggested that the transition may need an interplay of higher and leading twist effects. Shadowing is another of the possible explanations for the transition. The transition region is discussed fully in Sec. 8.

\subsubsection{Simple parametrizations}

We finish this introductory section by mentioning a few simple parametrizations of $F_{2}$ at small $x$ which have been inspired by various of the theoretical approaches mentioned above, but which find their ultimate justification in the quality of the fits to the data.

As we have seen, $\mathrm{pQCD}$ predicts $F_{2} \propto x^{-\lambda}$ at small $x$, where $\lambda$ is a constant for the naive solution to the BFKL equation (Eq. 169) and for the singular input solution to the DGLAP equations (Eq. 165), but $\lambda$ varies with $x$ and $Q^{2}$ for the

\footnotetext{
$\mathrm{v}$ In one scenario considered the twist- 4 contribution has an $x, Q^{2}$ dependence approximately proportional to the square of the leading twist contribution to $F_{2}$. This would imply that the usual forms used to describe higher twist contributions in fits to structure function data (see Sec. 3.4) are inadequate.
} 
commonly used non-singular input solution to the DGLAP equations in the DLLA (Eq. 164). The latter solution inspired the parametrization

$$
F_{2}=n_{i} x^{-\gamma} \sqrt{\frac{T}{\xi}}
$$

of De Roeck, Klein and Naumann246, where $\xi=\ln (1 / x)$ and $T=\ln \left(\alpha_{s}\left(Q_{0}^{2}\right) / \alpha_{s}\left(Q^{2}\right)\right)$. This form yields a $\chi^{2} / n d f$ of $109 / 167$ (using correlated systematic errors) in a fit to $\mathrm{H} 1(94)$ data in the kinematic region $x<0.1, Q^{2}>1.5 \mathrm{GeV}^{2}$, with two free parameters: $Q_{0}^{2}=0.365 \mathrm{GeV}^{2}$ and $\alpha_{s}=0.113$. This parametrization is essentially a simplification of the 2-loop result of the DAS approach, see Sec. 7.2.1

The simple parametrization

$$
F_{2}=a+b \ln \left(\frac{Q^{2}}{Q_{0}^{2}}\right) \ln \left(\frac{x_{0}}{x}\right)
$$

has been given by Buchmuller and Haidt 305, inspired by the search for a form which would saturate, but not violate, the Froissart bound at very low $x$. This double logarithmic form also represents the first term of the sum which produces the non-singular input solution to the DGLAP in the DLLA. This form yields a $\chi^{2} / n d f$ of $83 / 72$ (using uncorrelated systematic errors) in a fit to H1(94) data in the kinematic region $x<0.01, Q^{2}>5 \mathrm{GeV}^{2}$, with four free parameters $Q_{0}^{2}=0.5 \mathrm{GeV}^{2}$, $x_{0}=0.074, a=0.078$ and $b=0.364$. It has proved possible to extend this simple form to describe very low $Q^{2}$ data 306 , by making the substitution $\ln \left(Q^{2} / Q_{0}^{2}\right) \rightarrow$ $\ln \left(1+Q^{2} / Q_{0}^{2}\right)$. Both H1(94) data and H1(95)SVX data at very low $Q^{2}\left(Q^{2}\right.$ down to $0.35 \mathrm{GeV}^{2}$ ) can be well described in a fit to all data for which $x<0.005$. The parameter values are $Q_{0}^{2}=0.55 \mathrm{GeV}^{2}, x_{0}=0.04$, with intercept $a \simeq 0$, slope $b \simeq 0.45$.

De Roeck and De Wolf 307 have given the parametrization

$$
F_{2}=C_{0} \Gamma(\delta)\left(\frac{z}{2}\right)^{1-\delta} I_{\delta+1}(z)
$$

where $z=2\left(\frac{12}{\beta_{0}} \ln \left(\frac{1}{x}\right) T^{a}\right)^{\frac{1}{2}}, \Gamma$ is the Gamma function and $I_{\delta+1}(z)$ is a Bessel function of order $\delta+1$, where $\delta=\left(11+2 n_{i} / 27\right) / \beta_{0}$. This parametrization is inspired by the similarity between the $x$ dependence of $F_{2}$ at small $x$ and the $\sqrt{s}$ dependence of the average charged multiplicity in $e^{+} e^{-}$collisions. A fit with $\chi^{2} / n d f=265 / 231$ (using correlated systematic errors) to both H1(94) and ZEUS(94) data in the kinematic region $x<0.05,5<Q^{2}<250 \mathrm{GeV}^{2}$ has been obtained, with values of the two free parameters: $C_{0}=0.389, a=0.708,\left(Q^{2}=1 \mathrm{GeV}^{2}\right.$ and $\alpha_{s}=0.113$ are fixed). The success of the fit suggests that both deep inelastic scattering at small $x$ and $e^{+} e^{-}$annihilation can be adequately described by angular ordered QCD radiation in an essentially free phase space.

\subsection{Phenomenology: conventional DGLAP}

The success of the MRSR, CTEQ4 and GRV94 parametrizations already indicates that purely conventional DGLAP evolution summing only the leading (and next-to-leading) $\ln Q^{2}$ terms is adequate to describe the data down to $Q^{2}=1.5 \mathrm{GeV}^{2}$. 
This is surprising. When the HERA(92) data first revealed the steep rise of $F_{2}$ at low $x$ in 1993 this was taken as an indication that one was moving beyond conventional DGLAP into the region where the BFKL equation is applicable. The rise in $F_{2}$ at the lowest available $Q^{2}$ value, $Q^{2} \sim 10 \mathrm{GeV}^{2}$, was too steep to have been generated by DGLAP evolution from a conventional flat input at the conventional starting points for $Q^{2}$ evolution in the MRS and CTEQ parametrizations $\left(Q_{0}^{2}=4 \mathrm{GeV}^{2}\right.$ and $Q_{0}^{2}=2.56 \mathrm{GeV}^{2}$ respectively) and the GRV approach starting from a much lower input scale was considered controversial. The most popular explanation for the rise of $F_{2}$ at low $x$ was that given by the prediction of the AKMS group 291; $F_{2} \sim A x^{-\lambda_{L}} Q+F_{2}^{b g}$, where the first term represents a solution of the BFKL equation obtained by evolving down in $x$ from $x=0.01$, and the second term represents a soft non-perturbative term which describes larger $x$ data. Such solutions will be discussed further in Sec. 7.3

The MRS team had anticipated a need for non-conventional input by including a term $x^{-\lambda}$ in the gluon and sea distributions input at $Q_{0}^{2}$, in the MRSD parametrizations 35 . The Regge prediction, $\lambda=0$, from the soft Pomeron, represents the conventional input, but the alternative value, $\lambda=0.5$, from the BFKL Pomeron, was also tried. The data preferred a value $\lambda \simeq 0.3$, and this was initially taken as evidence for non-conventional BFKL evolution, since the prediction of $\lambda=0.5$ is rather naive, as we have already mentioned ${ }^{\mathrm{w}}$.

However the need for unconventional behaviour was rapidly challenged by the following considerations. Firstly, the data are only too steep to be generated from a flat input if the input scale is as high as $Q_{0}^{2} \simeq 4 \mathrm{GeV}^{2}$. If one lowers the input scale to $Q_{0}^{2}=1 \mathrm{GeV}^{2}$, one can fit the data with conventional DGLAP evolution and an input which is quite compatible with the conventional Regge soft Pomeron. The work of Ball and Forte219, which we discuss below, has been influential in establishing this idea, but such an approach had already been suggested by the success of the GRV91 parametrization which has a flattish shape $(\lambda \simeq 0.1)$ at $Q^{2}=1 \mathrm{GeV}^{2}$. The latest MRSR fits confirm the success of this idea for all data for which $Q^{2}>1.5 \mathrm{GeV}^{2}$. For example, MRSR4 has the flat input $\lambda_{g}=\lambda_{S}=0.04$ at $Q_{0}^{2}=1 \mathrm{GeV}^{2}$.

The success of these approaches derives from the DLLA prediction of de Rujula et al 270 given in Eqs. 161, 162. As we have already remarked, this is not strictly compatible with a power law dependence of $F_{2} \sim x^{-\lambda}$, but it can mimic a power law behaviour, over a limited region of $x, Q^{2}$ (see Eq. 164). Of course the exact value of $\lambda$ predicted for any $x, Q^{2}$ value depends on the chosen input values of $Q_{0}^{2}$ and $\Lambda$, but the variation of $\lambda$ with $Q^{2}$ (and $x$ ) will always have the same general shape (see Fig. 48). Thus we see that if we make parametrizations of the data which start conventional DGLAP evolution from a high value of $Q_{0}^{2}$ we will need a steeper input value of $\lambda$ than if we use a lower value of $Q_{0}^{2}$. Freedom to lower the input scale means that the conventional approach CAN be successful with a flattish input.

\footnotetext{
${ }^{\mathrm{w}}$ Of course if it is really necessary to use the BFKL equation, one cannot use parametrizations like ARS or CTEQ (which are based on the DGLAP equations) at all. However Kwiecinski et al 158 suggested that such a BFKL inspired input could represent a first approximation to the correct BFKL treatment.
} 
Secondly, even if we wish to maintain the somewhat larger input scale of $Q_{0}^{2}=$ $4 \mathrm{GeV}^{2}$, in order to avoid the non-perturbative region, the observation that one needs a steep input $\lambda$ at this scale is not necessarily an indication of unconventional behaviour. One has inadequate knowledge of the non-perturbative physics which determines the inputs, and hence one cannot exclude this as a reasonable input to conventional DGLAP evolution. Thus one should consider conventional explanations based on the solution of Eq. 160 applicable when one has a singular input glueg distribution: namely Eq. 165. Such a solution was chosen by Yndurain et al 145 for their early parametrizations and recent work, which is discussed further below, suggests that it can still provide a reasonable fit to present data272.

\subsubsection{Non-singular input gluon: double asymptotic scaling}

The work of Ball and Forte 19 on Double Asymptotic Scaling seeks to eliminate any dependence of the conventional DGLAP predictions on the form of the nonperturbative input. They define two new variables $\sigma$ and $\rho$, as follows

$$
\sigma=\sqrt{\ln \left(x_{0} / x\right) \cdot \ln \left(t / t_{0}\right)} ; \quad \rho=\sqrt{\ln \left(x_{0} / x\right) / \ln \left(t / t_{0}\right)}
$$

where $Q_{0}^{2}, x_{0}$ are low $Q^{2}$, high $x$ starting points. Taking the leading singularities in the splitting functions, as appropriate at low $x$ and high $Q^{2}\left(Q^{2}>Q_{0}^{2}, x<x_{0}\right)$, they derive that the gluon distribution, $g(\sigma, \rho)$, should rise exponentially with $\sigma$, for fixed $\rho$, and should be independent of $\rho$, for fixed $\sigma$, provided that asymptotic values of $\sigma$ and $\rho$ are reached. In other words, $\ln (g(\sigma, \rho)) / \rho$ should scale in both variables asymptotically, and this property is termed Double Asymptotic Scaling (DAS). Double Asymptotic Scaling is a property of conventionally evolved pQCD provided that the gluon distribution at $Q_{0}^{2}$ is NOT very singular. This caveat is the only remaining dependence of this prediction on the input distribution. The term 'very singular' is specified as $\lambda>\gamma / \rho \gtrsim 0.3$, where $\gamma=2 \sqrt{N_{c} / \beta_{0}}$.

Of course what we actually measure is $F_{2}(\sigma, \rho)$ rather than $g(\sigma, \rho)$, so these predictions need to be extended. Rescaling factors $R_{F}, R_{F}^{\prime}$ are defined as

$$
R_{F}^{\prime}=R \exp \left[\delta \frac{\sigma}{\rho}+\frac{1}{2} \ln (\sigma)+\ln \left(\frac{\rho}{\gamma}\right)\right] ; \quad R_{F}=R_{F}^{\prime} \exp (-2 \gamma \sigma)
$$

where $\delta=\left(11+2 n_{i} / 27\right) / \beta_{0}$ and $R$ is an arbitrary normalization factor. The prediction for $F_{2}$ is that $\ln \left(R_{F}^{\prime} F_{2}(\sigma, \rho)\right)$ should rise linearly with $\sigma$ for fixed $\rho$. In the rescaling factors, the term in $\ln (\rho / \gamma)$ takes care of the transition from the gluon distribution to $F_{2}$ and the terms in $\delta(\sigma / \rho)$ and $\ln (\sigma)$ take care of calculable subasymptotic effects, such that $\ln \left(R_{F}^{\prime} F_{2}\right)$ versus $\sigma$ at fixed $\rho$ lies on the SAME straight line independent of $\rho$. The slope of this straight line is predicted as $2 \gamma=2.4$, at leading order, for $N_{c}=3$ colours and $n_{i}=4$ flavours. A measurement of this slope is thus a measurement of the first coefficient of the QCD $\beta$ function $\beta_{0}$. The final rescaling term, $\exp (-2 \gamma \sigma)$, which defines $R_{F}$, removes this slope such that $\ln \left(R_{F} F_{2}\right)$ exhibits scaling in both $\sigma$ and $\rho$ (DAS).

The HERA(92) and HERA(93) data confirmed the predictions for a non-singular gluon input (and $x_{0}=0.1, Q_{0}^{2}=1 \mathrm{GeV}^{2}$ ). They exhibit Double Asymptotic Scaling 
for $\rho, \sigma ; \rho>1.8, \sigma>1.4$. However, the interpretation of the HERA(94) data needs a little more care, since these data include points at very low $Q^{2}, Q^{2}<5 \mathrm{GeV}^{2}$, for which the evolution length from a $1 \mathrm{GeV}^{2}$ starting scale may not be sufficient for DAS to be manifest. Even if we exclude these low $Q^{2}$ points, the data are now sufficiently precise that one can see that leading order DAS is not perfect, see Fig. 53. The slope of $\ln \left(R_{F}^{\prime} F_{2}\right)$ versus $\sigma$ is somewhat lower than $2 \gamma$ and $R_{F} F_{2}$ versus $\rho$ is not quite flat, it rises as $\rho$ increases.
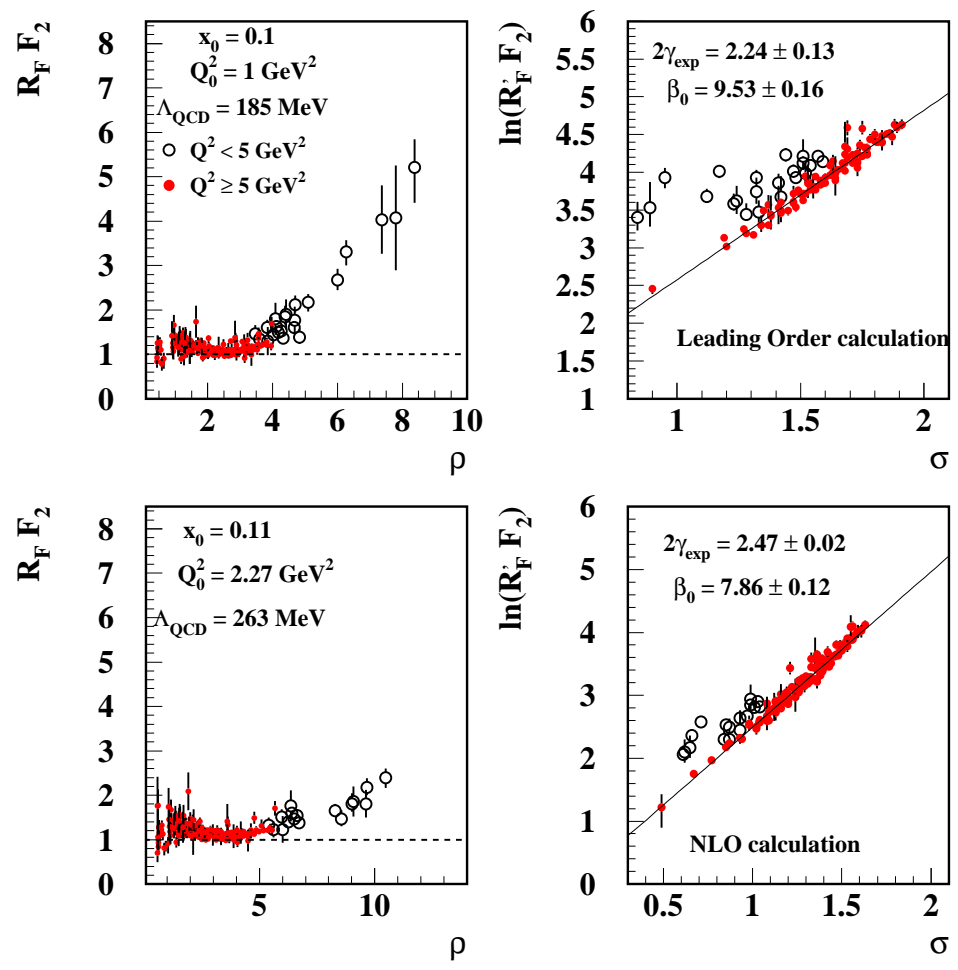

Fig. 53. The quantities $\ln \left(R_{F}^{\prime} F_{2}\right)$ versus $\sigma$ and $R_{F} F_{2}$ versus $\rho$ are illustrated for both LO (top) and NLO (bottom) evaluations. The data points are from the ZEUS(94) analysis, points with $Q^{2}<5 \mathrm{GeV}^{2}$ are indicated separately.

Agreement can be improved somewhat by the inclusion of subleading terms 308 , but Ball and forte find better agreement by extending their calculation to next-toleading order 309. Most of the NLO correction can be incorporated into a redefinition of the scaling variables such that one uses $\ln \left(\alpha_{s}\left(t_{o}\right) / \alpha_{s}(t)\right)$, rather than $\ln \left(t / t_{0}\right)$, where $\alpha_{s}$ is taken at second order. A slight change in the definition of the rescaling factor $R_{F}$ is also necessary

$$
R_{F}^{2-l o o p s}=R_{F}\left[1+\frac{\rho}{\gamma}\left(\epsilon \alpha_{s}\left(Q^{2}\right)-\epsilon^{\prime} \alpha_{s}\left(Q_{0}^{2}\right)\right)\right]
$$


where $\epsilon=\frac{1}{\beta_{0} \pi}\left(\frac{103}{27}+\frac{3 \beta_{1}}{\beta_{0}}\right)$ and $\epsilon^{\prime}=\epsilon+\frac{78}{\pi \beta_{0} \gamma^{2}}$. If one plots the data in terms of these new scaling variables one recovers the prediction that the slope of $\ln \left(R_{F}^{\prime 2-l o o p s} F_{2}\right)$ versus $\sigma$ is equal to $2 \gamma$ (with a somewhat higher input scale $Q_{0}^{2} \sim 2 \mathrm{GeV}^{2}$ ), but $\ln \left(R_{F}^{2-\text { loops }} F_{2}\right)$ is still not flat, see Fig. 53. However, although DAS is not perfect, the shape of $\ln \left(R_{F}^{2-l o o p s} F_{2}\right)$ CAN be described by a full (conventional) 2-loop calculation at the expense of reintroducing some dependence on the non-perturbative input. Data in the $Q^{2}$ region, $1.5<Q^{2}<5 \mathrm{GeV}^{2}$, is also described by such a full 2-loop calculation.

The procedure adopted in this NLO fit has already been described in Sec. 6.2 where it was used to extract $\alpha_{s}$. A fit to H1(94) data in the $\overline{\mathrm{MS}}$ scheme produces a $\chi^{2}$ of 80 for 169 data points, to be compared with the $\chi^{2}$ values given in Table 6 .

\subsubsection{Singular input gluon}

An alternative view predictions of conventional DGLAP is given by Yndurain and collaborators 272 310. They use the non-singular input solution of Eq. 160, such that $F_{2} \sim x^{-\lambda}$, with a fixed value of $\lambda$ equal to the input value, for all $Q^{2}$. The early work of Yndurain et al 145 gave a parametrization of the structure functions from an approximate analytic solution to the DGLAP evolution equations. As we have remarked, an exact analytic solution cannot be found for the entire $x, Q^{2}$ plane, but their solution was designed to be a 890 approximation in the very large and very small $x$ regions. More recent work 272 has pointed out that the low $x$ solution is of relevance to HERA data today. At small $x$ they predict (at leading order)

$$
F_{2}\left(x, Q^{2}\right) \simeq B_{S}\left[\alpha_{s}\left(Q^{2}\right)\right]^{-d_{+}(1+\lambda)} x^{-\lambda}
$$

where the only free parameters are, $B_{S}, \lambda$ and the QCD scale parameter $\Lambda$, since $d_{+}$is specified by the anomalous dimensions of quark and gluon operators. This form gives a good fit to the HERA(93) data $\left(x<0.01,8.5<Q^{2}<65 \mathrm{GeV}^{2}\right)$ with the same sort of values for the parameters, which were used in the ordinal fits to fixed target DIS data in 1980, namely $\lambda=0.38, B_{S}=2.7 \times 10^{-3}$ and $\Lambda=200 \mathrm{MeV}$ for four flavours.

When HERA(94) data are considered several further considerations arise. One must now fit data across a broad kinematic range. The form $\alpha_{s}^{-d_{+}(1+\lambda)}$ ensures that this term grows rapidly with $Q^{2}$ producing the strong spiking at low $x$ and high $Q^{2}$ which is seen in the data. However it also decreases as $Q^{2}$ decreases and sub-dominant terms could become important. The dominance of the singlet term at low $x$ is only overwhelming if we also have large $Q^{2}$, and since the HERA(94) data comprises points at $Q^{2} \lesssim 5 \mathrm{GeV}^{2}$, it is necessary to include the non-singlet contribution to $F_{2}$. The increased precision of the data also requires a fit at nextto-leading order. Most of the effect can be accounted for by evaluating $\alpha_{s}$ to second order, but there are somemodifications to the formulae. The full extended forms are detailed in references 310,311 . We note that the NLO corrections can be quite large and that the analytic NLO expressions are not as exact a solution to the NLO DGLAP equations as the analytic LO expressions are to the LO DGLAP equations. Finally, the range of $Q^{2}$ values is such that one is crossing flavour 
thresholds. The solutions are only meant to hold with respect to a constant number of flavours. If separate fits are made in different $Q^{2}$ regions: $n_{i}=3, Q^{2}<10 \mathrm{GeV}^{2}$ : $n_{i}=4,10<Q^{2}<100 \mathrm{GeV}^{2}: n_{i}=5, Q^{2}>100 \mathrm{GeV}^{2}$, then the $\chi^{2}$ values are $112 / 181$ for H1(94) data and 202/175 for ZEUS(94) data, and the $\lambda$ values are $\lambda=0.29,0.33,0.35$ respectively, for the different $Q^{2}$ ranges.

In principle, a picture which predicts constant $\lambda$ changing only at flavour thresholds should be experimentally distinguishable from the non-singular input prediction of a smooth variation of $\lambda$ with $Q^{2}$ (and $x$ ). However one must account for the way in which $\lambda$ can actually be measured. A fit of the form $x^{-\lambda}$ is made to data in the region $10^{-4}<x<10^{-2}$ for each $Q^{2}$ bin. Such an average $\lambda$ can also be evaluated for the theoretical predictions. This comparison is illustrated in Fig. 54 where one can see that in practice the predictions for singular and non-singular inputs are hard to distinguish.

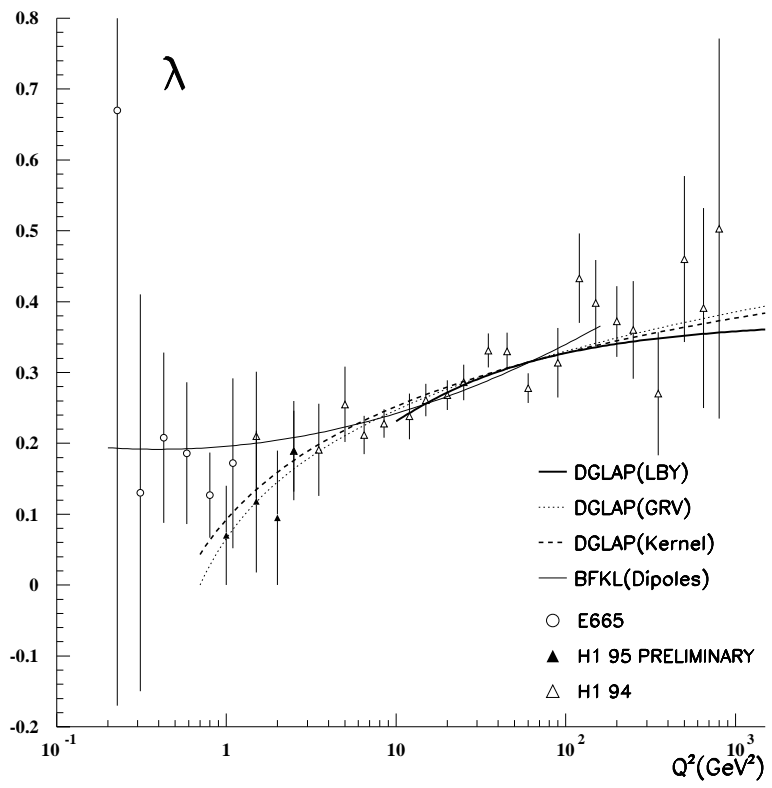

Fig. 54. The quantity $\lambda=\frac{d \ln F_{2}}{d \ln (1 / x)}$ as a function of $Q^{2}$ averaged over the $x$ range $10^{-4}<x<10^{-2}$, as evaluated from the DGLAP kernels, the parametrization of GRV, the parametrization of Lopez, Barreiro and Yndurain and from the BFKL dipole approach. Data from H1(94) and E665 are also shown for comparison.

The fact that a singular input, $F_{2} \sim x^{-\lambda}, \lambda \gtrsim 0.3$, is not compatible with the conventional soft Pomeron is not a problem for the present approach because it should be taken together with an alternatie model of total hadronic cross-sections, which was also suggested many years ago 312 , in which there is alyvays a contribution from hard scattering. This work has recently been updated 13 to provide a consistent picture of the transition region from DIS to photo-production, $Q^{2}=8.5 \rightarrow 0 \mathrm{GeV}^{2}$, 
which we discuss in Sec. 8. This approach also suggests that the higher $Q^{2}$ data, $Q^{2}>12 \mathrm{GeV}^{2}$, is best fitted by a combination of singular and non-singular inputs (corresponding to a soft and hard Pomeron respectively) at $Q_{0}^{2} \sim 2 \mathrm{GeV}^{2}$, each of which evolves in $Q^{2}$ in its own characteristic way: i.e.

$$
F_{2}\left(x, Q_{0}^{2}\right)=\left(B_{S} x^{-\lambda}+C_{S}\right)(1-x)^{\nu}
$$

where $B_{S}$ and $C_{S}$ are both constants and the larger $x$ data require the $(1-x)^{\nu}$ term. The $B_{S}$ term evolves to become $B_{S} \alpha_{s}^{-d_{+}\left(1+\lambda_{0}\right)}$ (at LO) and the $C_{S}$ term evolves according to the prediction of de Rujula et al. A fit of this combined approach to ZEUS data gives $\lambda_{0}=0.43$, larger than the values obtained from fits to the singular term alone, but interestingly consistent with the value $\lambda_{0}=0.47$ which is imposed by the condition that $F_{2} \rightarrow 0$ as $Q^{2} \rightarrow 0$ B13.

\subsection{Phenomenology: BFKL and beyond}

Phenomenological attempts to solve the BFKL equation are still somewhat underdeveloped because of the problems we referred to earlier namely: incorporating the running of $\alpha$ and introducing IR and UV cut-offs on the $k_{T}^{2}$ integrations. Many authors 314 315,299 have restricted themselves to addressing technical aspects and there is general agreement that no definitive work can be done until the consequences of the $N L L(1 / x)$ contributions have been fully worked out. Progress is being made on this matter at the time of writing 316 .

Much work has been done on the cut-offs and on their relationship to running $\alpha_{s}$. These limits need more careful consideration for BFKL than for DGLAP. Firstly because the lack of $k_{T}^{2}$ ordering means that the BFKL solution may diffuse into the infra-red region where perturbative calculations are unreliable 317 (given that $\alpha_{s}$ in the BFKL kernel is allowed to run, $\alpha_{s} \rightarrow \alpha_{s}\left(k_{T}^{2}\right)$, and $\alpha_{s}\left(k_{T}^{2}\right)$ can be very large for low $k_{T}^{2}$ ). Secondly because the DGLAP formulation ensures energy conservation order by order, but the BFKL formulation does not. Hence one has to impose it by the choice of the UV cut-off when working at finite order.

Thus the exact value of the power $\lambda_{L}$ for the BFKL gluon depends on the details of the full solution. Criticisms of the BFKL equation based purely on the naive value $\lambda_{L}=\frac{3 \alpha_{s}}{\pi} \ln 2$ are correspondingly inappropriate. 272318 In some early work it was suggested that the power $\lambda_{L} \simeq 0.5$ wguld be significantly reduced by consideration of the cut-offs 319 . Further work 291 315,314 has modified these conclusions. Askew et al 291 find that the effect of the IR cut-off alters the normalization of the resulting gluon but does not affect its slope very significantly. Forshaw et al 314 suggest that the effect of the UV cut-off on the predicted value of the slope is not very significant, basically because the running of $\alpha_{s}$ weights the integrand in the BFKL equation towards the infra-red region.

However there is another constraint which may be more significant than that of the UV cut-off 280. Consider a link in the gluon chain where the longitudinal momentum fraction decreases from $x / z$ to $x$ and the transverse momentum $k_{T}^{\prime}$ changes to $k_{T}$, with emission of a gluon of transverse momentum $q_{T}$. We require

$$
k_{T}^{2} / z>q_{T}^{2}
$$


in order that the virtuality of exchanged gluons is controlled by their transverse momenta. This implies that $k_{T}^{2} / z>k_{T}^{\prime 2}$, for any given value of $k_{T}$, and this is a much stronger constraint than that due to energy momentum conservation, which merely imposes, $Q^{2} / x \sim W^{2}>k_{T}^{\prime 2}$, provided that $Q^{2}>k_{T}^{2}$. If one considers the effect of this constraint on the solutions for the BFKL equation one finds that it is very significant for fixed $\alpha_{s}$. It modifies the asymptotic solution $x^{-\lambda_{L}}$, such that $\lambda_{L}$ is a far less steep function of $\alpha_{s}$. The usual value quoted $\lambda_{L} \sim 0.5$ is reduced to $\lambda_{L} \sim 0.3$. However, if one considers the case when $\alpha_{s}$ is running, the effect is less extreme because of the weighting of the integrand towards the infra-red. The application of this constraint goes some way towards accounting for $N L L(1 / x)$ effects. We consider this constraint further in Sec. 7.5 where it is applied to the CCFM and modified BFKL equations.

Recent work has concentrated on the consequences of incorporating running $\alpha_{s}$ into the BFKL equation, and the way in which it exacerbates the problem of drift into the non-perturbative infra-red region, such that the full solution to the BFKL equation may depend crucially on the IR boundary conditions and be essentially determined by non-perturbative physics 320.321 . This implies that it may be essential to include higher twist terms in our analysis of low $x$ physics as discussed by Bartels 300 . Indeed, if the transition to low $Q^{2}$ needs an interplay of higher and leading twist effects it is also reasonable to assert that higher twist terms must be non-negligible just above the transition region and thus that the success of leading twist DGLAP down to very low $Q^{2}$ is misleading. Furthermore Mueller 288 (see also Lev 289) has recently warned that leading twist pQCD may not be usable to very low $x$ unless $Q^{2}$ is larger than the mean parton transverse momentum. The OPE may breakdown because of diffusion into the infra-red in the non- $k_{T}^{2}$ ordered gluon ladder and this only gets worse if $\alpha_{s}$ is running,

Less pessimistically Camici and Ciafaloni 321 consider that one may use leading twist pQCD provided that $\alpha_{s} \ln (1 / x)$ is not too large. They have begun the work of putting together the $N L L(1 / x)$ corrections to the BFKL equation in order to understand running coupling effects 316 and they conclude that these corrections go in the direction of softening the BFKL Pomeron 322 . Recent work by several other authors points in the same direction $323,224275276$.

\subsubsection{Phenomenology of the BFKL equation}

The practical application of the BFKL equation to predict $F_{2}$ requires convolution of the gluon ladder with the quark box which connects it to the vector-boson probe, see Fig. 55. This is done using the $k_{T}$ factorization formula, which is a generalization of collinear factorization,

$$
F\left(x, Q^{2}\right)=\int_{x}^{1} \frac{d y}{y} \int \frac{d k_{T}^{2}}{k_{T}^{2}} f\left(y, k_{T}^{2}\right) \sigma^{b o x}\left(\frac{x}{y}, k_{T}^{2}, Q^{2}\right)
$$

where $f\left(y, k_{T}^{2}\right)$ is the unintegrated gluon density at the top of the gluon ladder and $\sigma^{b o x}$ denotes the quark box (and crossed box) contributions to the bosongluon subprocess. This convolution requires further consideration of the limits on 


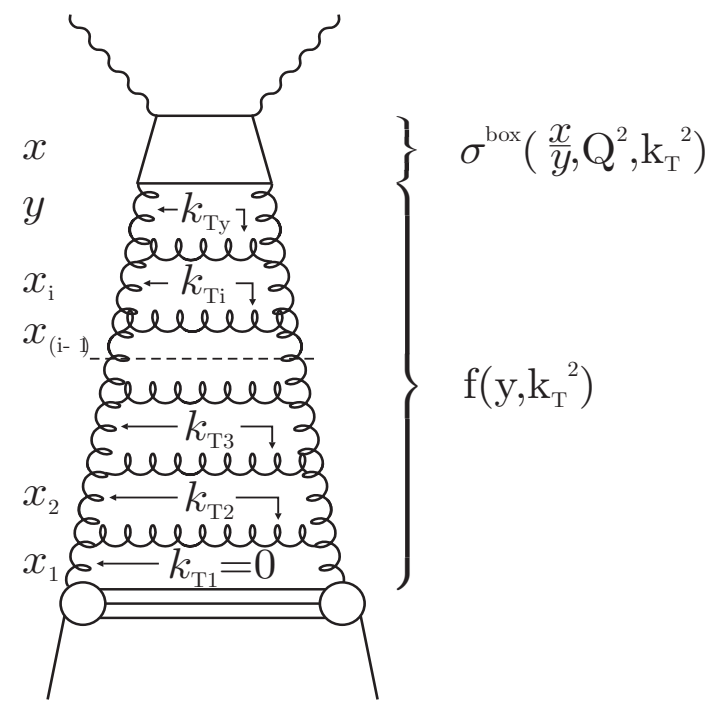

Fig. 55. Schematic representation of the convolution of the gluon ladder with the quark box

the $k_{T}$ integration. One expects the behaviour of the unintegrated gluon distribution, $f\left(x, k_{T}^{2}\right) \simeq x^{-\lambda_{L}}$, to feed through into the structure function. However, the hard physics which this embodies should be added to a background of conventional soft processes, which must be there to describe the behaviour of $F_{2}$ at larger $x$. Phenomenologically this gives a description of $F_{2}$ like that suggested by Eq. 184 . The combination of these two terms results in a smaller effective power of $\lambda$ for $F_{2} \sim x^{-\lambda}$, in the region of the present HERA data, than that coming from the BFKL contribution alone 291325.

An early phenomenological attempt to solve the BFKLequation incorporating many of the above considerations was given by the AKMS 291 group. This picture was successfully confronted with the early HERA data 326 . However it is unable to describe present HERA data which extend to much lower $Q^{2}$, since the effective $\lambda$ values necessary to describe data with $Q^{2}<10 \mathrm{GeV}^{2}$ are decreasing and the BFKL slope calculated by AKMS is simply too steep 327 . A modified BFKL equation which addresses the deficiencies of the AKMS approach has recently been developed and we discuss this in Sec. 7.5.

The colour dipole formulation of the BFKL equation 301 can be used to give a phenomenologically successful description of low $x F_{2}$ data One considers a $q \bar{q}$ (onium) state, within the proton, as a colour dipole. Since the valence quarks of the proton are irrelevant at small $x$, it is a reasonable physical picture to consider only the onia in the proton, these represent both sea quarks and gluons since the wave function of the onium state may evolve as it emits one, or more, soft gluons. If one pictures this development in impact parameter space, the original dipole of size $b$ becomes two colour dipoles $q g$ and $g \bar{q}$ of smaller size. Each of these dipoles can then branch independently, leading to a cascade of dipoles as $x$ gets smaller, explaining the rise in the number of dipoles (or gluons) at small $x$. To reveal the properties of the gluon distribution so generated, we must consider an interaction 
which probes the onium state. Theoretically it is simplest to consider onium-onium scattering. One derives a cross-section

$$
\sigma\left(b, b^{\prime}, Y\right)=\frac{8 \pi \alpha_{s}^{2} b b^{\prime}}{\sqrt{\pi k Y}} \exp \left(\lambda_{L} Y-\ln ^{2}\left(b^{\prime} / b\right) /(k Y)\right)
$$

where $b, b^{\prime}$ are the sizes of the two onia, $Y=\ln (1 / x)$ and $k=\alpha_{s} N_{c} 14 \zeta(3) / \pi$ (where the $\zeta$ function gives $\zeta(3)=1.202$ ). One notes that the high energy (small $x$ ) behaviour of the BFKL Pomeron $x^{-\lambda_{L}}$ is reproduced, and also that the dependence on the ratio $\left(b^{\prime} / b\right)$ will reproduce the dynamics of the BFKL equation such that there is diffusion in $k_{T} 302$.

In order to relate this to the structure functions in DIS we must consider a photon probe, of transverse size $1 / Q$. One first derives an expression for $F_{2}$ of an onium state of size $b$, and one then convolutes this with the probability to find a dipole of this size in the proton, which is essentially a non-perturbative function. However, since $b$ can be regarded as a factorization scale, the final result must be independent of $b$ so that we may set the $b$ dependence of this non-perturbative function to cancel out that in $F_{2}^{\text {onium }}$. For the detailed formalism see reference 328 , a simple representation of the solution is given by

$$
F_{2}=C a^{\frac{1}{2}} x^{-\lambda_{L}} \frac{Q}{Q_{0}} \exp \left(-\frac{a}{2} \ln ^{2}\left(\frac{Q}{Q_{0}}\right)\right)
$$

where $a=\left(\alpha_{s} N_{c} 7 \zeta(3) \ln (1 / x) / \pi\right)^{-1}, C$ is the normalization of the non-perturbative function and $Q_{0}$ is the non-perturbative scale $\left(Q_{0} \gg 1 / b\right)$.

Navelet et al 328 have used this form to explain the rise of $F_{2}$ at small $x$ seen in the HERA data. They restrict themselves to low $x\left(x<5 \times 10^{-2}\right)$ and moderate $Q^{2}$ $\left(1.5<Q^{2}<150 \mathrm{GeV}^{2}\right)$ data since they are not concerned with conventional physics at larger $x$, and the BFKL equation does not evolve with $Q^{2}$. They fit H1(94) data with 3 parameters: the non-perturbative scale $Q_{0}$, the normalization of the nonperturbative function which gives the probability of finding an onium in the proton, and the slope $\lambda_{L}$. They obtain a $\chi^{2}$ of 101 for 130 data points, giving parameter values $Q_{0}=0.63 \mathrm{GeV}$, for the non-perturbative scale, and $\lambda_{L}=0.28$, for the dominant contribution to the steep behaviour of $F_{2}$ at small $x$. Their prediction for the experimentally measurable quantity $\lambda=d \ln F_{2} / d \ln (1 / x)$ as a function of $Q^{2}$, averaged over the $x$ range $10^{-4}<x<10^{-2}$, is illustrated in Fig. 54 along with the measured values, and the predictions from the DGLAP equations with both singular and non-singular inputs. Very accurate data at low $Q^{2}\left(1<Q^{2}<10 \mathrm{GeV}^{2}\right)$ may be able to discriminate these theoretical pictures.

We note that within this picture $\lambda_{L}$ is predicted to be $12 \alpha_{s} \ln 2 / \pi$ as given in Eq. 169, so that the fitted value of $\lambda=0.28$ would give an effective value of $\alpha_{s} \simeq$ 0.11 , which corresponds well with the values derived from conventional analyses of DIS experiments, at the scale $M_{Z}^{2}$. However, most other work on the BFKL equation has assumed that a scale more like $Q^{2} \sim 5 \mathrm{GeV}^{2}$ would be appropriate, so that $\lambda_{L} \sim 0.5$. The authors interpret this discrepancy as indicating that the data yield an effective value of $\lambda_{L}$ for which the effect of cut-offs, running $\alpha_{s}$ and $N L L(1 / x)$ terms in the BFKL equation are already taken into account. 
Alternatively, Nikolaev et al 329 point out that whereas the solution of the BFKL equation is dominated by the leading eigenvalue, subleading values can be important non-asymptically, and introducing a running coupling constant can lead us into this region 330 . They have calculated the subleading eigenfunctions and singularities of the BFKL equation, with a running coupling constant, in the colour dipole representation. They describe $F_{2}\left(x, Q^{2}\right)$ by the simple Regge expansion

$$
F_{2}\left(x, Q^{2}\right)=\sum_{n} A_{n} F^{n}\left(Q^{2}\right)\left(\frac{x_{0}}{x}\right)^{\lambda_{n}}
$$

and find that they can fit data from E665, ZEUS(94) and H1(94), in the $x, Q^{2}$ range, $10^{-5}<x<10^{-1}, Q^{2}=1 \rightarrow 10^{3} \mathrm{GeV}^{2}$, with only 3-poles: $\lambda_{0}=0.4, \lambda_{1}=$ $0.22, \lambda_{2}=0.15$. The variation $d \ln F_{2} / d \ln (1 / x)$, with $Q^{2}$ (at small $x$ ) is also well described.

\subsection{Phenomenology: resummation of $\ln (1 / x)$ terms}

Much work has been done to establish that the resummation approach is really equivalent to the BFKL equation at small $x$ 331,332,333.334.335. The $L L(1 / x)$ solution of the BFKL equation (Eqs. 168, 169) can be rederived although it is approached only slowly. This approach has further important features. It introduces running $\alpha_{s}$ into the formalism, and it extends the original BFKL equation into the quark sector. One is essentially recasting the leading twist part of the BFKL $k_{T}$ factorization formula into a collinear form with $\ln (1 / x)$ terms included. It will simplify the discussion if we assume that we are working in a scheme like the DIS scheme, so that we may just consider the modifications to the splitting functions rather than needing to refer to both splitting functions and coefficient functions throughout. Most calculations have been performed in moment space so that it is actually the modified anomalous dimensions which are calculated.

The calculation of the gluon anomalous dimensions rederives the BFKL result at $L L(1 / x)$. However, it turns out that the approach to the steep asymptotic behaviour $x g(x) \simeq x^{-\lambda}, \lambda \simeq 0.5$, is rather slow, because the gluon anomalous dimension is given by the series

$$
\gamma_{g g, N}\left(\alpha_{s}\right)=\Sigma_{n=1}^{\infty} A_{n}\left(\frac{\alpha_{s}}{N}\right)^{n}
$$

and the coefficients $A_{n}$ are zero for $n=2,3,5$ due to strong cancellations coming from colour coherence 336 . Thus, in the HERA region, the resummation of $\ln (1 / x)$ terms to $L L(1 / x)$ in the gluon anomalous dimension has only a limited impact on predictions for $F_{2}$.

However, we may also calculate the quark anomalous dimensions with $\ln (1 / x)$ terms included. These are zero at leading order $L L(1 / x)$. (Recall that at small $x$ it was only the gluon splitting functions which were becoming singular, see Eq. 159.) However the next-to-leading $N L L(1 / x)$ contributions to the quark anomalous dimensions have been fully calculated (it is the $N L L(1 / x)$ contributions to the gluon anomalous dimensipns which are not yet fully understood) and they are found to be quite significant 331, since all the coefficients $A_{n}$ in the series

$$
\gamma_{q g, N}\left(\alpha_{s}\right)=\Sigma_{n=1}^{\infty} A_{n} \alpha_{s}\left(\frac{\alpha_{s}}{N}\right)^{n}
$$


are positive definite and large. Thus, the splitting function $P_{q g}$ is much steeper than in conventional DGLAP. Hence the quark sector, though formally subleading, turns out to have a significant impact on the predictions for $F_{2}$, such that $Q^{2}$ evolution from a flat to a steep quark distributions occurs much more quickly. Thus one may begin evolution at larger $Q_{0}^{2}$ values (so that $\alpha_{s}$ is small enough to trust perturbative QCD) and still achieve $F_{2}$ in agreement with HERA data at moderate $Q^{2}$.

Ellis, Hautmann and Webber 337 made the first quantitative investigations of resummation, giving a fit to HERA(93) data starting from flat gluon and sea input distributions at a scale of $Q_{0}^{2}=4 \mathrm{GeV}^{2}$. We do not discuss their work in detail since the HERA(94) data are manifestly not flat at this scale, however many of their results have more general relevance. They find that a steep behaviour of $F_{2}$ at low $x$ can be generated after a very short evolution length (from $Q^{2}=4 \rightarrow 8.5 \mathrm{GeV}^{2}$ ) only if $N L L(1 / x)$ effects in the quark sector are included, and that the size of this dominant contribution from the quark sector is very sensitive to the prescription chosen to impose momentum conservation.

Unlike the conventional DGLAP anomalous dimensions, the resummed anomalous dimensions do not obey momentum conservation automatically, hence it must be the higher order or subleading terms which restore it. Blümlein et al 338 have used various prescriptions for imposing energy and momentum conservation ${ }^{\mathrm{x}}$ and they conclude that the effects of subleading terms can be very significant. These authors have also considered the effects of resummation for both singlet and nonsinglet partons and for polarized and unpolarized structure functions. We will only discuss the unpolarized singlet case here ${ }^{\mathrm{y}}$.

The input gluon and sea quark distributions are taken to be of the form $x^{-0.2}$ at $Q_{0}^{2}=4 \mathrm{GeV}^{2}$, which gives a reasonable fit to $\operatorname{HERA}(94)$ data at this low $Q^{2}$. If a standard prescription (prescription $A$ of ref. 338 ) is used to impose momentum conservation then the effect of resummation is found to be very large compared to the NLO conventional DGLAP calculation. We may quantify this by taking the ratio of the predictions for the parton distributions when resummation terms are included to the corresponding predictions including only the conventional NLO terms, at the point $Q^{2}=10 \mathrm{GeV}^{2}, x=10^{-4}$. Including only $L L(1 / x)$ resummation one obtains a ratio of 1.3 for the gluon distribution and 1.03 for the sea quark distribution: adding the $N L L(1 / x)$ resummations in the quark sector changes the sea quark ratio dramatically to 3.3 and consequently the predictions for $F_{2}$ are strongly increased.

However, if a different prescription is used to impose momentum conservation then the effect of $L L(1 / x)$ and $N L L(1 / x)$ (for quarks) resummation terms is completely cancelled by the effective inclusion of higher order terms which this alternative prescription represents. The final result lies slightly below the conventional NLO prediction. These results are illustrated for prescriptions $A$ and $D$ of ref. 338

$\mathrm{x}$ Some of their prescriptions are disputed by Bojak and Ernst 327

y Resummation effects for unpolarized non-singlet structure functions are estimated to be only at the $1 \%$ level 
in Fig. 56.

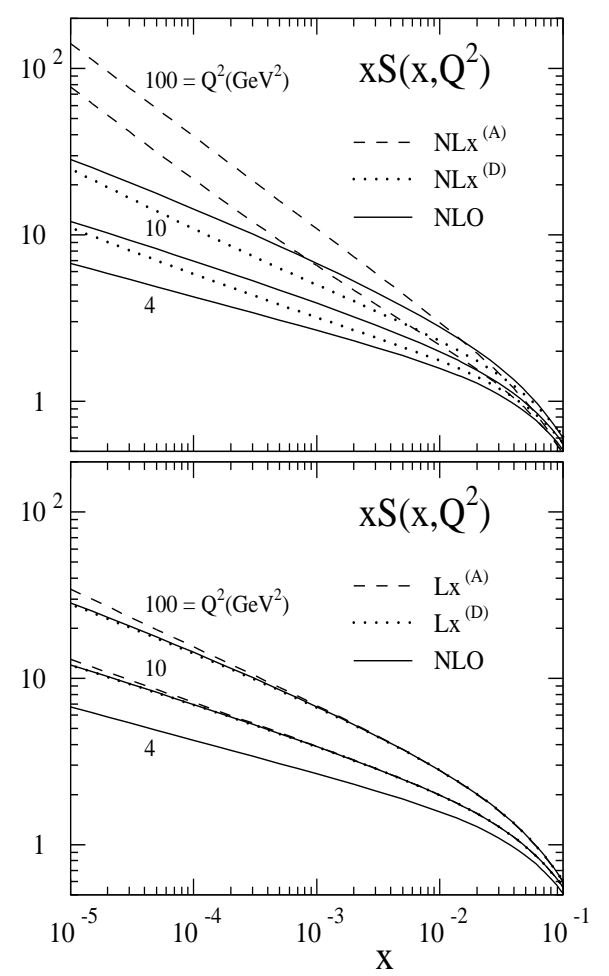

Fig. 56. Predictions from Blümlein, Riemersma and Vogt for the sea quark density as a function of $x$, for various different $Q^{2}$ values, including $L L(1 / x)$ resummation in the gluon sector (Lx), and $N L L(1 / x)$ resummation in the quark sector (NLx), for two different methods of implementing energy-momentum conservation labelled as (A) and (D)

Recently these authors have extended this analvsis 339 to include the calculation of the $N L L(1 / x)$ gluon anomalous dimension $\gamma_{g g} 274$. This has an almost negligible impact on the results for $F_{2}$, but it does soften the predictions for the gluon distribution considerably. However, it is not yet clear that the calculations of $\gamma_{g g}$ are complete to $N L L(1 / x) 34$ and the $N L L(1 / x)$ expressions for $\gamma_{g q}$ may not have the trivial relationship to $\gamma_{g g}$ which obtains at $L L(1 / x)$. These questions must be clarified before drawing further conclusions 341 .

From this discussion we conclude that the conventional BFKL equation, which deals only with the gluon sector, may not be very important at HERA, but extending the ideas of BFKL, by considering $\ln (1 / x)$ summation in the quark sector, certainly might be.

\subsubsection{Problems of scheme dependence}

We now consider ambiguities due to scheme dependence. If we work in renormalization schemes other than the DIS scheme then there is freedom in assigning 
the resummations to the coefficient functions or the splitting functions according to the renormalization scheme. This choice does not substantially alter our conclusions. However the choice of factorization scheme does. Differences in the speed of evolution (in either coefficient functions or splitting functions) can be absorbed into a difference in initial_conditions, i.e. we have the freedom to redefine the initial parton distributions 333 336.342. This is most easily seen by considering that we usually measure only $F_{2}$ and its scaling violations $d F_{2} / d \ln Q^{2}$. This does not provide enough information to disentangle perturbative from non-perturbative dynamics. Essentially we have $F_{2} \sim x q$ and $\frac{d F_{2}}{d \ln Q^{2}} \sim P_{q g} x g$ (at small $x$ ), thus steep behaviour of $d F_{2} / d \ln Q^{2}$ due to $\ln (1 / x)$ resummation in $P_{q g}$, could be accounted for by a redefinition of the gluon distribution $x g$, without redefining the quark distribution $x q$ and hence without affecting the prediction for $F_{2} 342$. Redefining the gluon distribution in this way (SDIS scheme) will allow us to continue to use conventional DGLAP evolution. This freedom of redefiniton can only be restricted by knowledge of the $N L L(1 / x)$ resummation in the gluon anomalous dimensions 343 or by a measurement of the gluon density at small $x$ which does not derive from the scaling violations of $F_{2}$, e.g. measurement of $F_{L}$ of $F_{2}^{c \bar{c}}$.

This scheme dependence explains why various authors 344 have considered resumming $\ln (1 / x)$ terms in calculating the splitting functions and coefficient functions and come to somewhat different conclusions as to the importance of such resummation. There is agreement that resummation effects can be very significant, but it is unclear that the HERA data require them. One can always modify the input distribution shapes and/or the input scale to fit the data. Ball and Forte 343 have made fits to HERA data using conventional NLO DGLAP evolution and compared them with fits including non-conventional resummation terms, in a variety of renormalization and factorization schemes. Fits to HERA(93) data with no resummation terms and a long evolution length had comparable $\chi^{2}$ to fits including resummation terms with a shorter evolution length. The work of Forshaw et al 345 comes to a very similar conclusion from a completely different choice of schemes. This lack of discrimination essentially comes from the fact that, for $Q^{2} \gtrsim 10 \mathrm{GeV}^{2}$, resummation corrections affect the predicted size of $F_{2}$ much more drastically than its predicted shape. This is no longer the case when calculations are extended to lower $Q^{2}$.

The high precision HERA(94) data, including the low $Q^{2}, 1.5<Q^{2}<5 \mathrm{GeV}^{2}$ region, considerably reduces the freedom of choice in input shape and input scale. Ball and Forte compare the conventional leading $\ln Q^{2}$ (or large $x$ ) expansion scheme with two other expansion schemes which perform resummation in slightly different ways: the leading $\ln (1 / x)$ (or small $x$ ) scheme and the double leading scheme in which the two logs are treated symmetrically (in the notation of Eq. 175 all terms with $p \geq q \geq 1, p \geq r \geq 0$ and $q+r \geq p \geq 1$ are summed at leading order, with an extra power of $\alpha_{s}$ at next-to-leading order). The latter two schemes are only adequate if $x$ is small, hence perturbative calculations are made conventionally down to an $x$ value, $x=x_{0}$, and then these schemes are used for lower $x$. The NLO fit in the conventional expansion scheme and $\overline{\mathrm{MS}}$ renormalization/factorization scheme, 
has already been described in Sec. 6.2. For the non-conventional expansion schemes, in addition to the free parameters, $\lambda_{S}, \lambda_{g}, \alpha_{s}$, the value of $x_{0}$ is also a parameter of the fit.

The detailed results depend on precisely which choice of schemes is made but one conclusion is common. The data do not favour unconventional (resummation) expansion schemes at all. Fig. 57 illustrates the variation of $\chi^{2}$ with $x_{0}$ for fits in the small $x$ and double leading expansion schemes. There is no minimum in $x_{0}$ indicating that the data do not need to use the unconventional schemes. The best $\chi^{2}$ is 80 to $169 \mathrm{H1}(94)$ data points, for a conventional NLO fit as applied in the $\overline{\mathrm{MS}}$ scheme. The other features of this fit are in broad agreement with the MRSR2 fit with a high value of $\alpha_{s}\left(\alpha_{s}=0.122\right)$ and values of $\lambda_{S}, \lambda_{g}$ such that the gluon distribution is valence-like and the quark distribution is rising moderately for low $Q^{2}\left(\simeq 1 \mathrm{GeV}^{2}\right)^{\mathrm{z}}$.

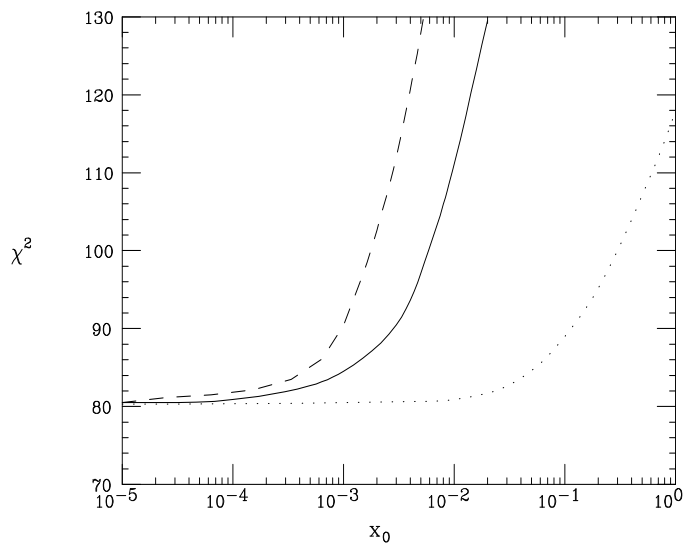

Fig. 57. The $\chi^{2}$ of the Ball and Forte fit as a function of $x_{0}$ in the $\overline{\mathrm{MS}}$ scheme. The different line types correspond to: double leading expansion with standard (full) or $Q_{0}$ (dashed) factorization and small $x$ expansion (dotted).

A similar but stronger conclusion, that resummation of $\ln (1 / x)$ terms cannot fit the HERA(94) data (for both ZEUS and H1) for low $Q^{2},\left(1.5<Q^{2}<5 \mathrm{GeV}^{2}\right)$ is made by Bojak and Ernst 346. 327. These authors have repeated the analyses of Ellis et al 33 and Forshaw et al 345 using the HERA(94) data, and they have extended the work of Ball and Forte to use more sophisticated parametrizations in their fits. However in all cases they conclude that the resummation terms introduce an $x$ dependence which is simply too steep to fit the lower $Q^{2}$ data, for which the slope of $F_{2}$ is flattening.

\footnotetext{
${ }^{\mathrm{z}}$ As usual one must beware of making direct comparisons of $\lambda$ values which are evaluated somewhat differently by different authors
} 


\subsubsection{Scheme independent calculations}

However these conclusions are disputed by work 347,248 which follows the suggestion of Catani 342 that one should try to formulate the dynamics of scaling violation entirely in terms of scheme independent quantities such as the measurable structure functions rather than parton distributions. Consider two observables such as $F_{2}$ and $F_{L}$ (or $F_{2}$ and $F_{2}^{c \bar{c}}$ ). One can write down the evolution equation

$$
\frac{d F_{2}\left(x, Q^{2}\right)}{d \ln Q^{2}}=\int_{x}^{1}\left[\Gamma_{22}\left(\frac{x}{y}, \alpha_{s}\right) F_{2}\left(y, Q^{2}\right)+\Gamma_{2 L}\left(\frac{x}{y}, \alpha_{s}\right) F_{L}\left(y, Q^{2}\right)\right]
$$

where we relate the scaling violations in the observables to the values of the same observables. The kernels $\Gamma$ are thus observables themselves. Because of the structure of this equation they may be thought of as physical anomalous dimensions. They can be related to the usual splitting factors and coefficient functions as evaluated in any scheme, and it can be established that the results turns out to be scheme independent as expected for an observable. The results do, of course, differ according to order of accuracy at which the evaluation is made, and in particular evaluations can be made conventionally, or including $\ln (1 / x)$ resummation terms, such that one may establish whether such resummation terms are necessary without scheme ambiguity.

Thorne 347248 goes further and argues that the fact that calculations for $L L(1 / x)$ terms are scheme dependent indicates that the method of incorporating these terms is incorrect. A correct, complete, leading order renormalization scheme consistent calculation for an observable quantity like a structure function (rather than a parton distribution) will naturally include $L L(1 / x)$ terms in the form of Catani's physical anomalous dimensions. Each of our usual expansion schemes (the conventional loop expansion in $\alpha_{s}$ or the small $x$ expansion in $\left.\alpha_{s} \ln (1 / x)\right)$ have shortcomings because some of the terms appearing at what we call higher orders are not actually subleading to terms which have already appeared. However the full set of terms in the combination of leading order expressions for both expansion schemes is genuinely leading order, and it is renormalization (and hence factorization) scheme independent. He labels this combined scheme the LORSC scheme aa and it is in this combined expansion scheme that the physical anomalous dimensions appear.

Thus the power counting of small $x$ logarithms is different for the physical anomalous dimensions than for the usual anomalous dimensions (and coefficient functions) and terms which are normally considered as classifiable as leading order or next-to-leading order get somewhat mixed up. An analysis to $L L(1 / x)$ in the physical anomalous dimensions (LORSC scheme) can be performed consistently using the $L L(1 / x)$ gluon anomalous dimensions $\gamma_{g g}$ and the $N L L(1 / x)$ quark anomalous dimensions $\gamma_{q g}$ which have already been calculated. An analysis to $N L L(1 / x)$ in the physical anomalous dimensions (NLORSC scheme) is not possible until the $N L L(1 / x)$ gluon anomalous dimensions $\left(\gamma_{g g}\right.$ and $\left.\gamma_{g q}\right)$ have been fully evaluated.

One further feature of Thorne's LORSC fit is notable. He demands that any deviation of the structure function from a flat Regge type behaviour at small $x$ must

aa In some of the earlier references the LORSC scheme is called the LO(x) scheme. 
Table 9. $\chi^{2}$ per data point, for QCD fit de to DIS datasets in the LORSC scheme and in the conventional NLO scheme by Thorne 347248 and by MRS 37

\begin{tabular}{cccc}
\hline Data & LORSC & NLO & MRSR2 \\
\hline BCDMS F $F_{2}^{p}$ & $181 / 174$ & $218 / 174$ & $320 / 174$ \\
CCFR $(93) F_{2}$ & $59 / 66$ & $48 / 66$ & $56 / 66$ \\
CCFR $(93) x F_{3}$ & $48 / 66$ & $39 / 66$ & $47 / 66$ \\
NMC ratio & $142 / 85$ & $137 / 85$ & $132 / 85$ \\
$N M C(97) F_{2}^{p}$ & $122 / 129$ & $131 / 129$ & $135 / 129$ \\
$N M C(97) F_{2}^{d}$ & $114 / 129$ & $107 / 129$ & $99 / 129$ \\
$Z E U S(94) F_{2}^{p}$ & $253 / 204$ & $281 / 204$ & $308 / 204$ \\
$H 1(94) F_{2}^{p}$ & $123 / 193$ & $145 / 193$ & $149 / 193$ \\
$E 665 F_{2}^{p}$ & $63 / 53$ & $63 / 53$ & $63 / 53$ \\
\hline
\end{tabular}

come from perturbative effects. Thus his inputs are non-perturbative functions, flat at small $x$, convoluted with functions of the physical anomalous dimensions which are determined by evolution from a non-perturbative scale $A_{L L}$ to the input scale $Q_{0}^{2}$. Only when $Q_{0}^{2}=A_{L L} \simeq 1 \mathrm{GeV}^{2}$, are the inputs purely non-perturbative. He then requires insensitivity of the fits to $Q_{0}^{2}$. This approach predicts a relationship between the small $x$ inputs for $F_{2}, d F_{2} / d \ln Q^{2}$ and $F_{L}$ such that one can no longer redefine inputs freely.

Thorne 34248 has performed fits to H1(94) and ZEUS(94) data, and also to the data of BCDMS, NMC(97), E665 and CCFR(93), which establish that one does get a better $\chi^{2}$ working in the LORSC scheme than with conventional NLO fits such as those of MRSR. The quality of the fit is shown in Fig. 58 and the $\chi^{2}$ are presented in Table 9, where the $\chi^{2}$ for the MRSR2 fits are also given for comparison. Note that the $\chi^{2}$ values for MRSR fits to NMC data differ from those already given in Table 6, since Thorne has used the new NMC(97) data 95, and has re-evaluated the MRSR $\chi^{2}$ for these data. A fairer comparison between the LORSC scheme and the conventional NLO treatment may be obtained by making a conventional NLO fit in exactly the same circumstances as the LORSC fit, i.e. including the same data sets and using the same programme but leaving out resummation terms. Thorne has done such a fit and it is included in Table 9 as the 'NLO' fit. One sees that the LORSC fit is an improvement over the NLO fit overall. As expected most of the improvement is at low $x$ : if a cut $x<0.1$ is made on all data sets then the LORSC fit gives a $\chi^{2}$ of 483 for 548 data points, whereas the NLO fit gives a $\chi^{2}$ of 554 . The NLO fit is superior for higher $x$ data where $\alpha_{s}^{2}$ terms are important, a more definitive comparison should come when an NLORSC fit becomes available.

The major criticism of the approach of the present section is that it deals with an integrated gluon density. One has lost some of the physics of the non-integrated gluon ladder. Thus such an approach cannot address the problem that $k_{-}^{2}$ values in a non- $k_{T}$ ordered gluon ladder may drift into the infra-red region 34 , 321 . However Camici and Ciafaloni 321 consider that Thorne's approach should be valid provided that $\alpha_{s} \ln (1 / x)$ is not 'too' large. A related criticism is that one also cannot evaluate the effect of the imposition of constraints such as Eq. 185 and the approach does 


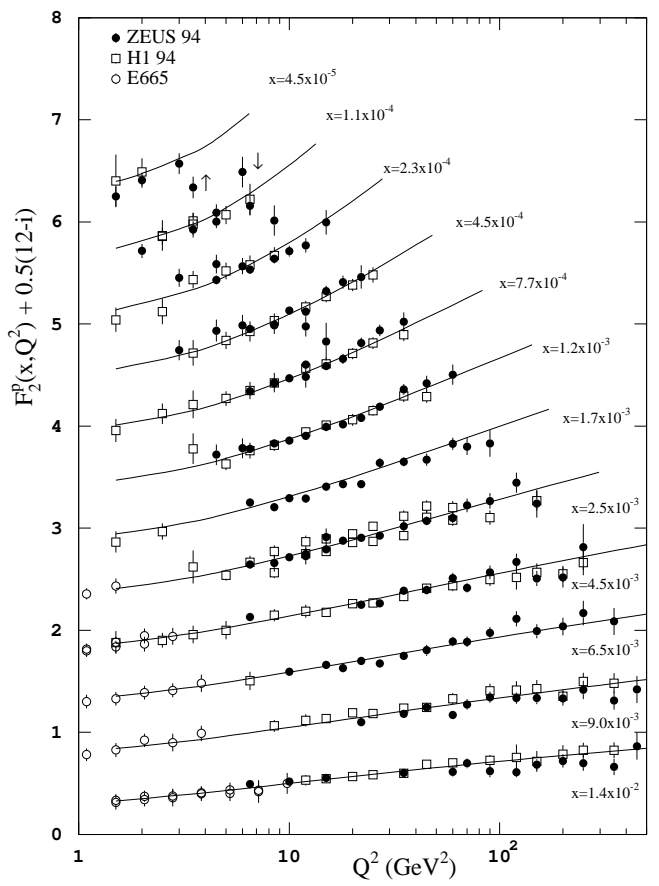

Fig. 58. The solid line shows the LORSC fit to HERA(94) and E665 data on $F_{2}$ as a function of $Q^{2}$ for different $x$ bins. For clarity of display 0.5(12-i) is added to the value of $F_{2}$ each time the value of $x$ is decreased, for $i=1,12$. The ZEUS data are renormalized by 1.015 to produce the best fit. Data are assigned to the $x$ value closest to the experimental $x$ bin.

not consider higher twist operators 284. Thus it is interesting to consider the CCFM equation or the modified BFKL equations which preserve a richer physical structure.

\subsection{Phenomenology: CCFM and modified BFKL equations}

\subsubsection{The CCFM equation}

The CCFM equation 277 is defined in terms of a scale dependent unintegrated gluon density $f\left(x, k_{T}^{2}, Q^{2}\right)$ which specifies the chance of finding a gluon with longitudinal momentum fraction $x$ and transverse momentum $k_{T}$ at the scale $Q^{2}$. The practical application of the CCFM equation to predict $F_{2}$ involves many of the same considerations as the application of the BFKL equation. One has to incorporate running of $\alpha_{s}$ and to consider the UV and IR cut-offs on the $k_{T}$ integration (although this is somewhat more straightforward since the angular ordering constraint acts as an infra-red regulator and we may avoid the severe problems of drift into the non-perturbative region which beset the implementation of the BFKL equation 281). One also has to convolute the CCFM gluon with the quark box at the top of the gluon ladder and add the result to the soft background appropriate at large $x$.

The phenomenology has been explored by Kwiecinski et al 279. They obtain 
$f\left(x, k_{T}^{2}, Q^{2}\right)$ by approximating the full CCFM equation appropriately at small $x$. Accordingly, the splitting functions retain only the $1 / x$ terms and the effect of singlet quarks on the gluon evolution is ignored. Running $\alpha_{s}$ is incorporated by using $\alpha_{s}\left(k_{T}^{2}\right)$. One must input a non-perturbative gluon distribution to the equation, at a starting scale $Q_{0}^{2}$. Kwiecinski et al chose the form $f\left(x, k_{T}^{2}, Q_{0}^{2}\right) \sim$ $3(1-x)^{5} \exp \left(-k_{T}^{2} / Q_{0}^{2}\right)$, i.e. flat at low $x$ and with a narrow $k_{T}$ distribution. The starting scale is chosen as $Q_{0}^{2}=1 \mathrm{GeV}^{2}$.

The resulting gluon distribution can be compared with that which would be obtained conventionally from the DGLAP equations, or from the BFKL equation, starting from the same input. The CCFM unintegrated distribution has a $k_{T}^{2}$ dependence which broadens and develops a significant tail as $x$ decreases, however diffusion in $k_{T}$ is reduced compared to the BFKL equation, and correspondingly sensitivity to the IR cut-off is reduced. The angular ordering in CCFM also introduces a dependence of the unintegrated gluon on the scale $Q^{2}$, which is significant at low $Q^{2}$, whereas the BFKL gluon acquires $Q^{2}$ dependence only from the $k_{T}^{2}$ integration. Hence the CCFM gluon evolves faster in $Q^{2}$ than the BFKL gluon.

Thus, if we compare the integrated gluon distributions, the CCFM gluon distribution is much steeper at small $x$ than that generated by conventional DGLAP in the DLLA, but less steep than the BFKL gluon at moderate $Q^{2}$, evolving to become very similar at high $Q^{2}$. These features are illustrated in Fig. 59. Characterizing the steepness of the gluon's slope in terms of the form $x^{-\lambda}$, we see that the CCFM slope $\lambda$ has a stronger $Q^{2}$ dependence than that of the BFKL, such that for low $Q^{2} \sim 10 \mathrm{GeV}^{2}$ it is smaller by $\sim 0.1$, whereas at higher $Q^{2}$ one has $\lambda \sim 0.5$ just as for the BFKL result. Kwiecinski et al conclude that the part of the $N L L(1 / x)$ effects which are included in the CCFM equation modify the steep behaviour of the $L L(1 / x)$ BFKL result such that the onset of this form is more delayed for CCFM. The recent work of Bottazzi et al comes to similar conclusions 281 .

Kwiecinski et al also use the CCFM gluon to make predictions for $F_{2}$, using the $k_{T}$ factorization theorem and imposing an infra-red cut-off, $k_{0}^{2} \sim 1 \mathrm{GeV}^{2}$, on the $k_{T}^{2}$ integration. The CCFM prediction for $F_{2}$ is then added to a background from the conventional soft Pomeron in order to connect smoothly to the larger $x$ region. The result was compared to HERA(93) data and it gave a good description, lying between the MRSA' and GRV predictions. Note that it is NOT a fit but a prediction, although there is some freedom in the choice of the non-perturbative input shape and input scale, the background shape and the infra-red cut-off value. It is encouraging that physically reasonable choices give a reasonable description.

In the preceding discussion no consideration has been given to the constraints coming from energy-momentum conservation, which impose a UV cut-off on $k_{T}$ integrations, or to the constraint embodied in Eq.185, which is more restrictive than angular ordering at small $z$, since it automatically embodies the angular ordering constraint $\left(Q^{2}(1-z)^{2} / z^{2}>q_{t}^{2}\right)$ until $Q^{2}$ falls below $k_{T}^{2}$. These two constraints were considered in a separate publication of Kwiecinski et al 280 (see also 349 ). As a result the slope $\lambda$ for the CCFM gluon is further reduced by $\sim 0.1$, and the 


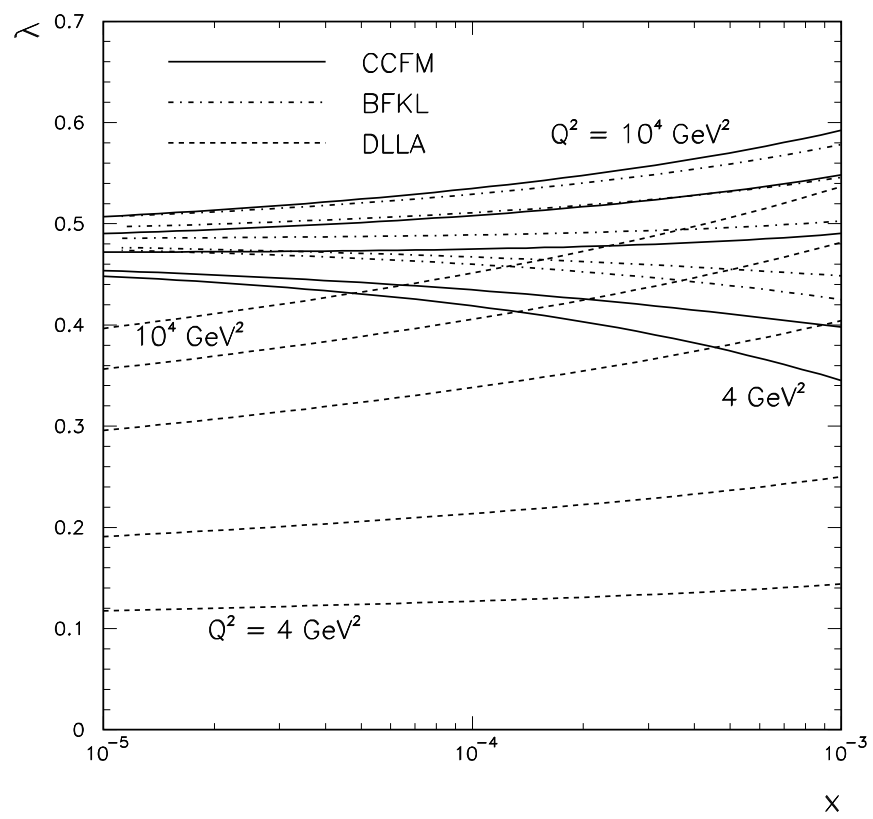

Fig. 59. The effective values of $\lambda, x g \sim x^{-\lambda}$, for CCFM, BFKL and the DLLA, for $Q^{2}=$ $4,10,10^{2}, 10^{3}, 10^{4} \mathrm{GeV}^{2}$ versus $x$.

predictions for $F_{2}$ show a reduced slope with respect to $Q^{2}$ as well as with respect to $1 / x$. A reasonable description of HERA(93) data can still be obtained. However, the authors have not pursued this approach to make detailed comparisons with HERA(94) data, preferring to regard the work as a comparison of the behaviour of the CCFM, BFKL and DGLAP equations within the present incomplete state of theoretical understanding.

\subsubsection{Modified BFKL equations}

The modified BFKL equation of Lie82 introduces $Q^{2}$ dependence into the BFKL equation essentially from applying a UV cut-off at $Q^{2}$ to integrals over loop momenta and from introducing a fixed IR cut-off $Q_{0}^{2}$ on virtual gluon emission. A $Q^{2}$ dependent unintegrated gluon distribution is obtained which is used to predict $F_{2}$ using the $k_{T}$ factorization theorem and a form of the gluon input which is flat at $Q_{0}^{2}=1 \mathrm{GeV}^{2}, x_{0}=0.1$, with a narrow $k_{T}$ distribution, similar to that used for the CCFM solution discussed above. The resulting integrated gluon distribution has a $Q^{2}$ dependent slope at small $x$ given by $x g \sim\left(\frac{x}{x_{0}}\right)^{-\alpha_{s} \ln \left(Q^{2} / Q_{0}^{2}\right)}$ and correspondingly the rise in $F_{2}$ at small $x$ is less steep at smaller $Q^{2}$ than that predicted by the unmodified BFKL equation. Thus reasonable agreement with the data is obtained in the $Q^{2}$ range $8<Q^{2}<20 \mathrm{GeV}^{2}$ (only 4 flavours are considered). More recently 283 this work has been extended to relax the strong ordering in $x$ usually assumed 
in the derivation of the BFKL equation, with the result that the predicted gluon distribution saturates (and thus obeys the unitarity bound) at small $x,\left(x \lesssim 10^{-4}\right)$.

Kwiecinski, Martin and Stasto 284 have developed a modified BFKL equation which is applicable over all $x$ and $Q^{2}>1 \mathrm{GeV}^{2}$. They develop a unified BFKL and DGLAP equation for the gluon by adding to the BFKL equation terms which include leading order DGLAP evolution (i.e. terms which are leading in $\alpha_{s}$ but subleading in $\alpha_{s} \ln (1 / x)$ ) as follows

$$
\begin{aligned}
f\left(x, k_{T}^{2}\right)=f^{0}\left(x, k_{T}^{2}\right) & +\frac{3 \alpha_{s}\left(k_{T}^{2}\right)}{\pi} k_{T}^{2} \int_{x}^{1} \frac{d z}{z} \int_{k_{0}^{2}} d k_{T}^{\prime 2} K\left(k_{T}^{\prime 2}, k_{T}^{2}\right) f\left(\frac{x}{z}, k_{T}^{\prime 2}\right) \\
& +\frac{3 \alpha_{s}\left(k_{T}^{2}\right)}{\pi} \int_{x}^{1} \frac{d z}{z}\left(\frac{z}{6} P_{g g}(z)-1\right) \int_{k_{0}^{2}}^{k_{T}^{2}} \frac{d k_{T}^{\prime 2}}{k_{T}^{\prime 2}} f\left(\frac{x}{z}, k_{T}^{\prime 2}\right) \\
& +\frac{\alpha_{s}\left(k_{T}^{2}\right)}{2 \pi} \int_{x}^{1} d z P_{g q}(z) \Sigma\left(\frac{x}{z}, k_{T}^{2}\right)
\end{aligned}
$$

The first term is the starting distribution. The second term embodies the usual BFKL equation integrated from the IR cut-off $k_{0}^{2}$ but the kernel $K\left(k_{T}^{\prime 2}, k_{T}^{2}\right)$ is subject to the constraint given in Eq. 185. The third term in $P_{g g}$ gives the usual DGLAP convolution of the gluon density with the splitting function but the gluon density has been split into an infra-red piece $\frac{x}{z} g\left(\frac{x}{z}, k_{0}^{2}\right)$ which is incorporated in the starting distribution and a piece which is obtained from integrating over the unintegrated gluon density. The -1 allows for the contribution already included in the BFKL summation. The fourth term in $P_{g q}$ is the usual DGLAP contribution allowing quarks to contribute to the evolution of the gluon. The starting distribution is taken to be $f^{0}\left(x, k_{T}^{2}\right)=\frac{\alpha_{s}\left(k_{T}^{2}\right)}{2 \pi} \int_{x}^{1} d z P_{g g}(z) \frac{x}{z} g\left(\frac{x}{z}, k_{0}^{2}\right)$, given entirely in terms of a flat non-perturbative gluon distribution, $\frac{x}{z} g\left(\frac{x}{z}, k_{0}^{2}\right)=N\left(1-\frac{x}{z}\right)^{\beta}$, so that the rise in $F_{2}$ at small $x$ is generated entirely by perturbative dynamics (either from $\ln (1 / x)$ or $\left.\ln Q^{2}\right)$.

Before we can solve the above equation we must specify the quark distribution $\Sigma\left(y, k_{T}^{2}\right)$. This is given by a further coupled equation

$$
\begin{aligned}
\Sigma\left(x, k_{T}^{2}\right)=S^{a}(x)+V\left(x, k_{T}^{2}\right) & +\int_{k_{0}^{2}}^{k_{T}^{2}} \frac{d k_{T}^{\prime 2}}{k_{T}^{\prime 2}} \frac{\alpha_{s}\left(k_{T}^{2}\right)}{2 \pi} \int_{x}^{1} d z P_{q q}(z) S\left(\frac{x}{z}, k_{T}^{\prime 2}\right) \\
& +\sum_{i} \int_{k_{0}^{2}}^{\infty} \frac{d k_{T}^{\prime 2}}{k_{T}^{\prime 2}} \int_{x}^{1} \frac{d z}{z} S_{i}^{b o x}\left(z, k_{T}^{\prime 2}, Q^{2}\right) f\left(\frac{x}{z}, k_{T}^{\prime 2}\right)(194) \\
& +\sum_{i} \int_{x}^{1} \frac{d z}{z} S_{i}^{b o x}\left(z, k_{T}^{\prime 2}=0, Q^{2}\right) \frac{x}{z} g\left(\frac{x}{z}, k_{0}^{2}\right)
\end{aligned}
$$

The first, fourth and fifth terms in the above equation express the application of the $k_{T}$ factorization theorem in different kinematic regions. Recall that this theorem is usually used to predict $F_{2}$ from the unintegrated gluon distribution. However, it is really only the sea quark part of $F_{2}$, which is predicted since the cross-section $\sigma^{b o x}$ in Eq. 186 involves only the $g \rightarrow q \bar{q}$ transition. In the present formalism one wants to evaluate $S\left(x, k_{T}^{2}\right)$, the sea quark distribution, rather than its contribution 
to $F_{2}$ and this is why the cross-sections are denoted as $S^{b o x}$ rather than $\sigma^{b o x}$. This evaluation implicitly involves an integration over the transverse momentum $\kappa$ of the exchanged quark in the box, thus it involves three separate contributions according to the relative sizes of $\kappa, k_{T}$ and $k_{0}$. The contribution from the infrared region $k_{T}^{2}, \kappa^{2}<k_{0}^{2}$ is parametrized by a form appropriate for light sea quarks $S^{a}(x)=C_{P} x^{-0.08}(1-x)^{8}$ as suggested by the soft non-perturbative Pomeron. The contribution from the region $k_{T}^{2}<k_{0}^{2}<\kappa^{2}$ is given by a convolution of the integrated gluon distribution $\frac{x}{z} g\left(\frac{x}{z}, k_{0}^{2}\right)$ with $\sigma^{b o x}$ for each flavour of quark (5th term). Finally, when $k_{T}^{2}, \kappa^{2}>k_{0}^{2}$, the $k_{T}$ factorization theorem may be used fully perturbatively (4th term). Note that the charm component of the sea is evaluated perturbatively in all regions. Thus far the $k_{T}$ factorization theorem has been used to play the role of $P_{q g} \otimes x g$ convolution in the DGLAP equations. To complete the evaluation of $\Sigma\left(x, k_{T}^{2}\right)$ one must also add the $P_{q q} \otimes x q$ convolution as given by the third term (for sea quarks) and a parametrization of the valence quarks $V\left(x, k_{T}^{2}\right)$. (This is mainly determined by fixed target data and the GRV94 parametrization is used). In summary, one no longer just adds a background of conventional soft processes to the result from the $k_{T}$ factorization theorem. The physics of conventional processes is incorporated in a more thorough manner.

Thus we have two coupled equations which can be solved for the unintegrated gluon distribution and the singlet quark distribution. From these $F_{2}$ (in the DIS scheme) and the integrated gluon distribution can easily be obtained. This gluon distribution exhibits a much less steep behaviour than that resulting from the unmodified BFKL equation. In fact it is quite similar to that of the conventional DGLAP parametrization of MRSR2 and its slope varies with $Q^{2}$ similarly. The result for $F_{2}$ exhibits a rise at small $x$ which softens as $Q^{2}$ decreases just as observed. There are only 2 variable parameters in the fit, $N$ and $\beta$, which specify the flat non-perturbative gluon input. The fits are not sensitive to the valence parameters or indeed to the Regge form chosen for the light quark sea. The normalization $C_{P}$ of the light quark sea is determined from the momentum sum rule and $\alpha_{s}\left(M_{Z}^{2}\right)=0.12$ is kept fixed. The input scale is $k_{0}^{2}=1 \mathrm{GeV}^{2}$. Fits are made to HERA(94) data and to $\mathrm{NMC}(97)$, BCDMS, E665 data, with a resulting $\chi^{2} / n d f=1.07$ and parameters $N=1.57, \beta=2.5$. This fit is an improvement on the fully conventional fit of the MRSR2 parametrization to the same data, which has $\chi^{2} / n d f=1.12$. Finally we note that it is important that the kinematic constraint of Eq. 185 be included in the analysis. A fit without this constraint results in too steep a slope of $F_{2}$ at small $x$ and moderate $Q^{2}$ and a much poorer $\chi^{2} / n d f(=1.8)$. It is also interesting that it is the constrained fit which gives the better description of the WA70 prompt photon data which sample the gluon at high $x$. The quality of the fit and the effect of the constraint are illustrated in Fig. 60.

Fig. 60 also compares the present approach to a DGLAP NLO calculation, where the DGLAP calculation replaces the unified DGLAP/BFKL approach in both the quark and gluon sectors, or only in the gluon sector. All the calculations start from the same flat gluon input at scale $1 \mathrm{GeV}^{2}$. From this comparison we can see 

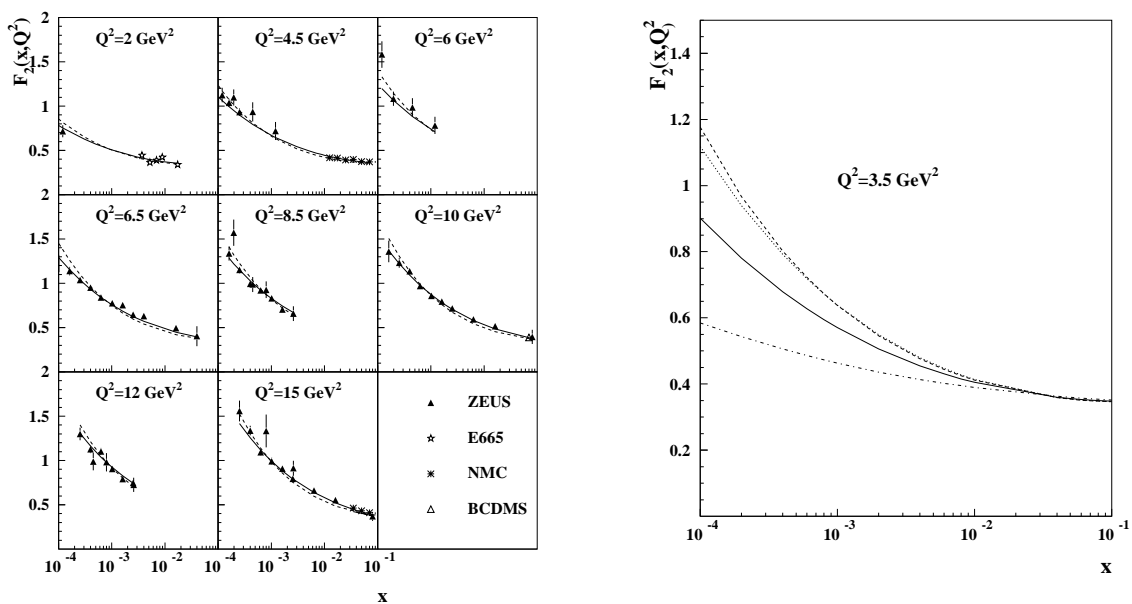

Fig. 60. lhs: the Kwiecinski, Martin and Stasto description $F_{2}$ data (from EMC, BCDMS, $\mathrm{NMC}(97)$, ZEUS(94)) at small $x$ using $f\left(x, k_{T}^{2}\right)$ evaluated with (continuous curves) and without (dashed curves) the kinematic constraint of Eq. 185: rhs: the dotted and dot-dashed curves are obtained using DGLAP in the gluon sector and in both the gluon and the quark sectors respectively, whereas the continuous and dashed curves are as for the lhs.

that the use of the unified approach is most significant in the quark sector. In the previous subsection we found that $\ln (1 / x)$ resummation was also most significant in the quark sector. A further similarity in the non-conventional approaches of these two subsections is that one starts with a non-perturbative flat gluon distribution (and flattish sea distribution) at a scale of about $Q^{2} \sim 1 \mathrm{GeV}^{2}$ and the rise in $F_{2}$ is generated completely perturbatively, rather than being input or generated from a much lower starting scale. If we focus instead on the differences between the current approach and the unmodified $(L L(1 / x)$ BFKL equation, we see that the partial inclusion of $N L L(1 / x)$ effects, which the introduction of running $\alpha_{s}$ and the application of the constraint of Eq. 185 represent, has lead to a softening of the BFKL Pomeron.

\subsection{Summary and outlook for $p Q C D$ at low $x$}

To sum up, there has been much debate as to whether non-conventional QCD evolution is required by the HERA data. Good fits to the data can be obtained from all approaches described in this section if one restricts the $Q^{2}$ range to $Q^{2} \gtrsim 10 \mathrm{GeV}^{2}$. However it is more of a challenge to fit data, in the $Q^{2}$ range, $1<Q^{2}<10 \mathrm{GeV}^{2}$. The approaches which meet this challenge are: conventional DGLAP evolution with soft gluon input, BFKL in the colour dipole approach, unconventional DGLAP including $\ln (1 / x)$ resummation worked out in the LORSC scheme and the modified BFKL equation which incorporates $\ln Q^{2}$ and $\ln (1 / x)$ summation. The extension 
of the validity of perturbative QCD into what was previously thought of as the non-perturbative region $\left(Q^{2} \lesssim 4 \mathrm{GeV}^{2}\right)$ is surprising, the transition region to nonperturbative physics appears to be very narrow. However we must be mindful of the warnings issued by Levine7d (that non-linear shadowing effects may be a more importantmodification to the conventional evolution equations than $\alpha_{s} \ln (1 / x)$ terms), Bartels 300 (that higher twist effects should be important above the transition region) and Mueller 288 (that the pQCD may not be appropriate at small $x, Q^{2}$ because of diffusion into the infra-red). In a recent review of higher order QCD corrections Van Neerven 350 has also emphasized that considering only the most singular parts of the $\ln (1 / x)$ corrections can give misleading results. It is also possible that further higher order and subleading terms (requiring non-perturbative calculations) could be important 321 .

The $\chi^{2}$ of the conventional fits are comparable to those of the unconventional fits. Thus it is hard to establish that non-conventional QCD processes are definitely needed to describe HERA data on $F_{2}$ and its scaling violations. However, if they are not needed, this itself requires some explanation since we have clearly reached a region where $\alpha_{s} \ln (1 / x) \sim 1$, so that conventional expansions in $\alpha_{s}$ should no longer be reliable. We gather together here a few possible reasons which we have mentioned in passing. It may be because of cancellations between higher order or subleading terms and the leading $\ln (1 / x)$ contributions: we already know that there are extraordinary cancellations such that there are no $\ln ^{2}(1 / x)$ terms in the singlet sector 351 338 , and that the coefficients of many of the $L L(1 / x)$ terms in the gluon anomalous dimensions are zero. It may be because the dominant effect of the $\ln (1 / x)$ resummation is already included in the conventional sum when it is taken to NLO such that the term with $n=m=2$ is included. It may be because the leading behaviour of the BFKL equation (Eqs. 168, 169) is approached very slowly as derived in $\ln (1 / x)$ resummation calculations. It may be because shadowing effects mask the effect of $\ln (1 / x)$ terms. It may be because the effect of incorporating running $\alpha_{s}$ and higher order effects into the BFKL formalism softens the BFKL Pomeron 322. Work on the CCFM equation and on modified BFKL equations points in this direction. Haakman et al 323 even contend that the solution of the BFKL equation is modified so that the BFKL Pomeron behaves instead like the Pomeron corresponding to the result of de Rujula et al 270 (see Eq. 164) which softens as $Q^{2}$ decreases. Ball and Forte 324 contend that if the BFKL equation is derived from a high energy factorization theorem it does not result in a hard Pomeron at all, and the conventional and non-conventional sums give results which are very alike for the structure function $F_{2}$. Camici and Ciafaloni 316 consider that a full understanding of $N L L(1 / x)$ effects would also lead to an understanding of the transition to soft physics as $Q^{2} \rightarrow 0$.

We should be able to gain some answers by measuring different structure functions. The freedom to redefine the parton distributions can only be maintained if we consider only $F_{2}$ and $d F_{2} / d \ln Q^{2}$. If we use the parton distributions so determined to predict the high energy behaviour of other observables such as $F_{L}$ or $F_{2}^{c \bar{c}}$, 
then these predictions will differ if the parton densities are redefined, and accurate measurements of such quantities coyld lead to a resolution of the ambiguities 336 . For example, both Forshaw et al 345 and Ball and Forte 351 found that including the $\ln (1 / x)$ resummation terms alters the relative normalizations of the quark and gluon distributions One requires a larger gluon to fit the $F_{2}$ data if one does not include resummation terms because the starting scale has to be lower and there is more time for evolution. Thus if one compares predictions for $F_{L}$ based on parton distributions extracted from a conventional fit to predictions for $F_{L}$ based on parton distributions extracted from a fit including $\ln (1 / x)$ resummation terms one will see a significant difference: the conventional fit will predict a larger $F_{L}$. Within the conventional framework one may relate the gluon distribution at small $x$ both to $F_{L}$ and $F_{2}$ (Eq. 153) and to $d F_{2} / d \ln Q^{2}$ and $F_{2}$ (Eq. 151) and refs. 185, 186. Thus it is clearly possible to obtain a set of self consistent relationships between $F_{L}$ and $F_{2}$ and $d F_{2} / d \ln Q^{2}$ which will be violated if non-conventional effects enter. Such relationships have been given by in a simple form by Kotikov and Parente 352 (see also ref. 353 ).

Many of the non-conventional analyses give predictions for $F_{L}$ : BFKL dipoles, CCFM and the modified BFKL equation of Kwiecinski Martin and Stasto, and the scheme independent analysis of Thorne. All of these non-conventional predictions share the common feature of being lower than the conventional predictions. Predictions from the analysis of Thorne are shown in Fig. 61. There is a similar discrepancy between conventional and non-conventional predictions for $F_{2}^{c \bar{c}}$. The prediction from the CCFM analysis of Kwiecinski et al is shown in Fig. 62.

Present measurements of $F_{L}$ and $F_{2}^{c \bar{c}}$ were given in Secs. 5.3, 5.4. Most of the measurements of $F_{L}$ are not in the region of the $x, Q^{2}$ plane which would allow us to use them to establish or refute the need for $\ln (1 / x)$ terms. We need to measure $F_{L}$ at HERA energies to reach small $x$ and moderate $Q^{2}$. We note that the glimpse of $F_{L}$ given by H1 101 is not a model independent measurement and thus cannot be used to discriminate the need for $\ln (1 / x)$ terms, see ref. 125 for a full discussion. Thus, as explained in Sec 5.3, we need to run HERA at different beam energies 23 , or to use the ISR events 126.128 . It is unlikely that HERA will run at lower beam energies in the near future. However, measurement of $F_{2}^{c \bar{c}}$ and of $F_{L}$ using ISR events should become possible with the proposed upgrades to the HERA machine, which will yield very large integrated luminosity.

In view of the difficulty in distinguishing signals of non-conventional behaviour in current structure function measurements it will also be useful to look for other signals of non-conventional behaviour, for example in the hadronic final state.

\subsection{Searching for BFKL effects in the hadron final state}

Two characteristic features of BFKL dynamics are the absence of strong $k_{T}$ ordering along the gluon chain ${ }^{\mathrm{bb}}$, and the consequent growth of the cross-section as

\footnotetext{
$\mathrm{bb}$ However, note that even the lack of strong $k_{T}$ ordering which is taken to be a signal for BFKL evolution is already there to some extent in conventional NLO, for one pair of gluons in the ladder.
} 


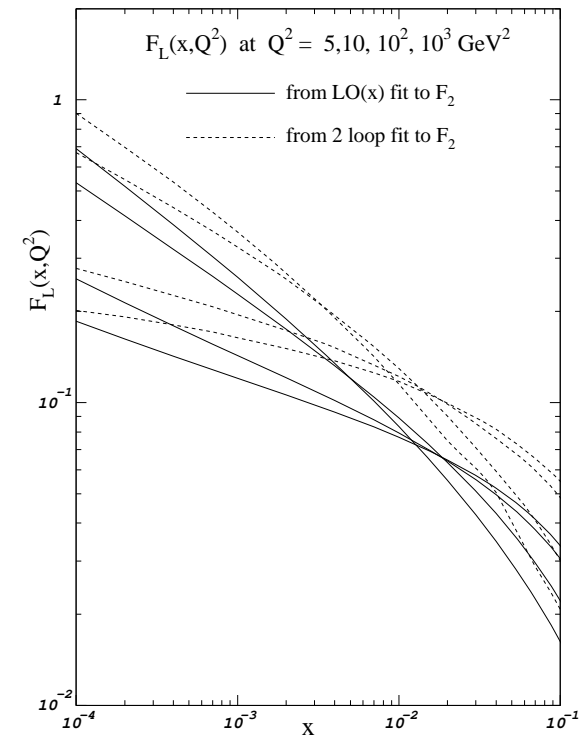

Fig. 61. The prediction for $F_{L}$ from the renormalization scheme consistent calculation including $\mathrm{LL}(1 / \mathrm{x})$ terms (called $\mathrm{LO}(\mathrm{x})$ fit), compared to the conventional prediction from an NLO (2-loop) fit.

$\exp (\lambda \Delta y)$ where the rapidity interval, $\Delta y=\ln \left(x_{1} / y\right)$, is defined between the gluons at either end of the gluon ladder, with longitudinal momentum fractions $x_{1}$ and $y$ (see Fig. 11). The larger the value of $\Delta y$ the more dominant are the leading $\ln (1 / x)$ contributions.

Thus for events at low $x$, hadron production in the region between the current jet and the proton remnant should be sensitive to the difference between BFKL and DGLAP dynamics. In the leading log DGLAP scheme the parton cascade follows strong ordering in transverse momentum $k_{T n}^{2} \gg k_{T n-1}^{2} \gg \ldots \gg k_{T 1}^{2}$, whereas there is no ordering in transverse momentum 354 for the BFKL scheme. The transverse momentum follows a kind of random walk in $k_{T}$ space: the $k_{T i}$ value is close to the $k_{T i-1}$ value, but it can be both larger or smaller 355 . As a consequence, BFKL evolution is expected to produce more gluons with substantial transverse energy $E_{T}$ in the region between the struck quark and the proton remnant, for low $x$ events, compared to DGLAP evolution 356.

Some processes which may reveal these effects are: deep inelastic events with a measured forward jet; total transverse energy flow and single particle $p_{T}$ distributions in DIS events; production of a pair of jets at large rapidity interval in hadronic collisions or in DIS, and the azimuthal decorrelations between these jets. The 'forward' direction at HERA is the proton direction. We discuss only the former two predictions in detail, (see Fig. 63), since there is, as yet, no data from DIS on the 


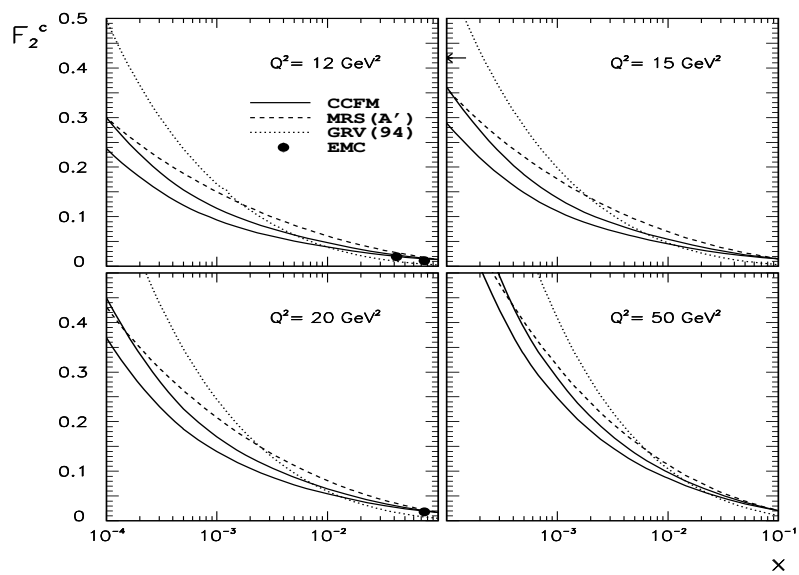

Fig. 62. The prediction for $F_{2}^{c \bar{c}}$ from the CCFM equation for two values of the charmed quark mass $m_{c}=1.4 \mathrm{GeV}$ (upper) and $m_{c}=1.7 \mathrm{GeV}$ (lower) compared to that from the GRV and $\mathrm{MRSA}^{\prime}$ parametrizations. EMC charm data are shown for comparison

latter processes. For a discussion of the interesting effects which may be observed in future and possible present indications in non-DIS data, see references 357.359 .360 .36

To make comparisons of such predictions with data we must account for hadronization of the final state partons and for the experimental acceptance and resolution. This is usually done within Monte Carlo models, based upon QCD phenomenology. We briefly discuss the three main models used. Firstly, the LEPTO Monte Carlo incorporates the QCD matrix elements to first order and takes higher order parton emissions into account approximately by using the concept of parton showers i.e. branching processes of partons. This model is often called the MEPS model. The branching processes are based on DGLAP dynamics and the partons generated in the leading log parton showers are strongly ordered in $k_{T}$. Hadronization is done within the LUND string model 662 . Secondly the HERWIG Monte Carlo has a similar QCD treatment with leading log parton showers, but has a different hadronization model involving a clustering algorithm 363. Thirdly the ARIADNE Monte Carlo calculates multi-gluon radiation by emitting gluons from a chain of independently radiating colour dipoles spanned by colour connected partons 8 . This is often called the CDM model (or CDMBGF since the boson-gluon fusion process has to be added from the QCD matrix elements). It has no strong $k_{T}$ ordering and should thus give some indication of BFKL behaviour, although it does not explicitly use the BFKL equation. Hadronization is again done by the LUND string model.

Monte Carlo programmes which incorporate BFKL evolution have recently been developed by Schmidt 364 and Orr and Stirling 365, and a Monte Carlo implementation of the CCFM equation in the linked dipole chain LDC model is under development 366, but these Monte Carlos have not yet been used extensively to confront the data.

Apart from these Monte Carlo implementations of QCD based models, there are 


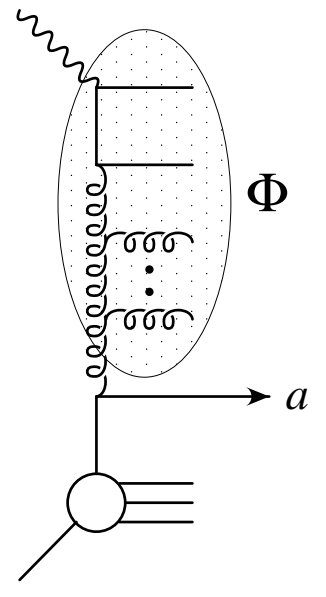

(a) DIS + jet

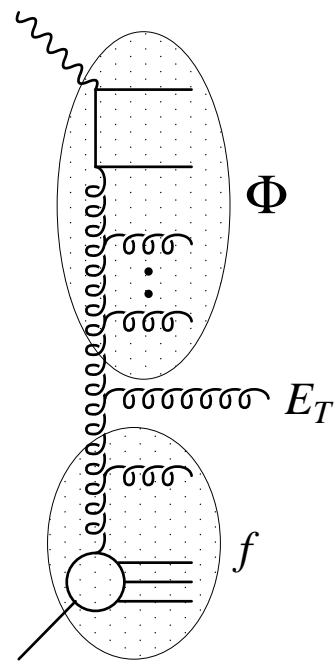

(b) $\mathrm{E}_{\mathrm{T}}$ flow

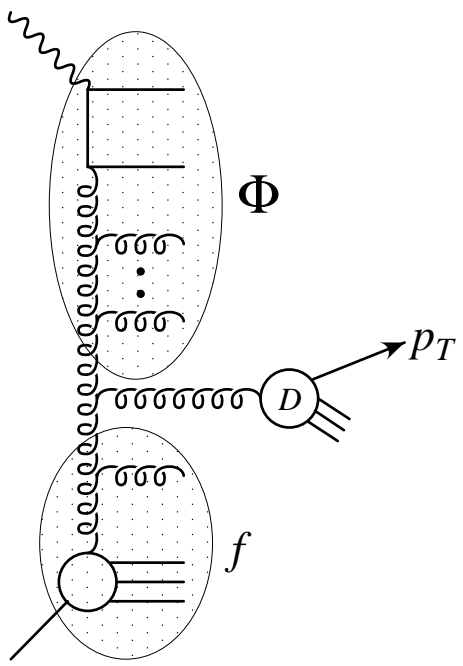

(c) Single particle spectrum

Fig. 63. Processes which test BFKL dynamics: a) DIS with a forward jet: b) $E_{T}$ flow in DIS: c) single particle $p_{T}$ spectra in DIS.

some analytic calculations, based on (approximate) solutions of the BFKL equation. These are generally calculations at the parton level, and thus they should not really be compared directly with the data. Such calculations are used to give some indication of the differences between BFKL and conventional dynamics, before hadronization. Thus they serve to give a first indication of the effect of BFKL dynamics on the variable under study.

\subsubsection{Energy flows}

We first discuss transverse energy flow in DIS events. If we suppose that there is no strong $k_{T}$ ordering then we expect to find more transverse energy, $E_{T}$, emitted in the central region between the current jet and the proton remnant (in the lab frame this is the experimentally problematic forward region) than would result from conventional evolution. Parton level calculations incorporating BFKL dynamics yield a fairly flat central plateau with $E_{T} \simeq 2 \mathrm{GeV}$ per unit of rapidity in the HERA regime 356 . Early observations 367 of such a level of $E_{T}$ were taken as indicating a need for BFKL dynamics, particularly since comparison of the data with LEPTO and ARIADNE favoured the latter. However, these observations led to a reconsideration of the hadronization prescriptions in both Monte Carlos and comparisons with more recent versions (LEPTO6.4368, ARIADNE(4.07) 369) show that one can produce $E_{T}$ values $\simeq 2 \mathrm{GeV}$ even from conventional dynamics. In LEPTO the new features included in the model are soft colour interactions (SCI) 370 , which are intended to describe rapidity gap events (usually thought to be of diffractive 
origin) without introducing the concept of a Pomeron and its structure function, and a modified sea-quark/remnant treatment (SQT), giving a smoother transition from BGF events to events where the photon interacts with a sea quark.

Results for the energy flow versus pseudorapidity $\eta$ are shown in the hadronic centre of mass system (CMS) in Fig. 64, for two different $x$ bins at a $Q^{2} \sim 14$ $\mathrm{GeV}^{2}$. Overlaid are model predictions, which broadly agree with the data. It is of interest to check the $x$ dependence of the $E_{T}$ for $\eta<0$ (the proton direction). This is illustrated in Fig. 65 where the H1 171 and ZEUS 372 data from the 1994 run are displayed. The average value of $E_{T}$ in the central region $\left(-0.5<\eta^{*}<0.5\right)$ is plotted as a function of $x$ for $Q^{2}=14 \mathrm{GeV}^{2}$, and compared with model predictions. As $x$ decreases the values of $\left\langle E_{T}\right\rangle$ increase. This observation can be explained by the Monte Carlo models, but in different ways. Models based on DGLAP evolution with strong $k_{T}$ ordering produce $60-80 \%$ of the $E_{T}$ in hadronization. Hadronization compensates for the fact that at the parton level the $\left\langle E_{T}\right\rangle$ actually decreases as $x$ decreases, contrary to the trend in the data. The importance of hadronization is demonstrated by the predictions from LEPTO without the newly introduced ingredients SCI and SQT. This prediction is much below the data and has clearly a different $x$ dependence.

By contrast the CDM model with no $k_{T}$ ordering produces more $E_{T}$ in the central region, with the right $x$ dependence, at parton level, so that only $30-40 \%$ extra needs to be supplied by hadronization. The $x, Q^{2}$ dependence of the $\left\langle E_{T}\right\rangle$

also agrees with the analytical BFKL calculation 356 , but this calculation was only made at the parton level, thus we see that it leaves room for only $10-20 \%$ more of $\left\langle E_{T}\right\rangle$ to come from hadronization ${ }^{\text {cc }}$.

Hence we are now in a situation that all models can account for the data, despite differences in the underlying parton dynamics: the mean $E_{T}$ can be made to agree by exploiting as yet unconstrained variations in hadronization models. This unsatisfactory situation may be clarified by considering $E_{T}$ distributions rather than mean $E_{T}$. One expects $E_{T}$ from hadronization to be limited, whereas high $E_{T}$ should come from partonic activity. Thus the tails of the $E_{T}$ distributions are of interest. First studies indeed show that for fixed $Q^{2}$ the high $E_{T}$ tail gets harder with decreasing $x$, and similarly for fixed $x$ it gets harder with increasing $Q^{2}$. This is demonstrated more clearly when studying $p_{T}$ spectra of charged particles as discussed in the next section.

\subsubsection{Charged particle transverse momentum spectra}

It has been demonstrated with Monte Carlo studies that a more direct measurement of the partonic activity in the hadronic final state could be the measurement of single particle transverse momentum $\left(p_{T}\right)$ spectra 373 . A harder $p_{T}$ tail is expected when there is unordered partonic activity in the ladder. H1 has made a measure-

\footnotetext{
cc Even at the parton level BFKL calculations are not yet fully reliable sinfe the $N L L(1 / x)$ corrections have yet to be worked out, for example study of the CCFM equation 281 indicates that angular ordering significantly reduces transverse energy flow.
} 


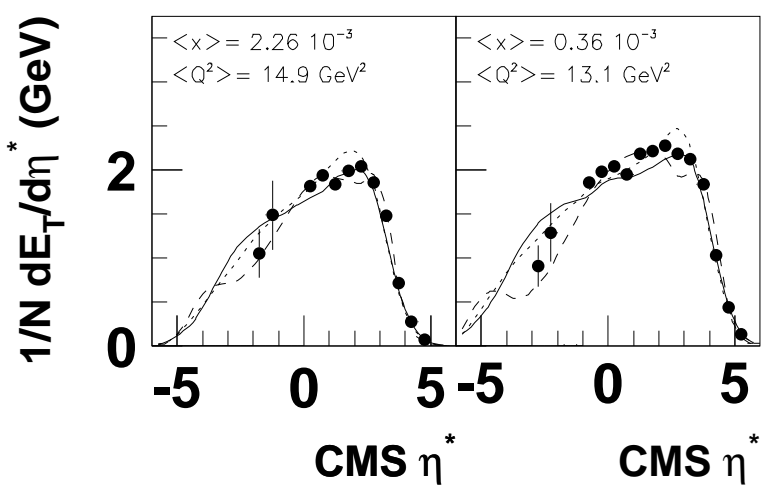

- H1 94 prel. ARIADNE HERWIG LEPTO

Fig. 64. The $E_{T}$ flow versus $\eta$ in the hadronic CMS for H1(94) preliminary data. The proton direction is to the left. The data are compared to the models CDM, ARIADNE and HERWIG (see text).

ment of the charged particle $p_{T}$ spectra in the CMS region $0.5<\eta<1.5$, limited by the acceptance of the tracker detectors 374 . The result is shown for two $x$ bins at $Q^{2} \simeq 14 \mathrm{GeV}^{2}$, together with model predictions, in Fig. 66. The errors reflect the statistical and systematic uncertainties. Clearly at high $x$ all models agree with the data, whereas at small $x$ the ARIADNE prediction is closest to the data. LEPTO and HERWIG produce significantly too few large $p_{T}$ tracks.

In Fig. 67 the result of a theoretical calculation 375 with and without BFKL evolution is shown. It consists of a BFKL calculation at the parton level folded with experimentally measured fragmentation functions. The normalization of the BFKL calculations has been made using the preliminary forward jet cross-sections (see next subsection). Good agreement with the data is observed for the calculation which includes BFKL evolution.

\subsubsection{Forward jets}

Next we consider DIS events with a measured forward jet. Jets are expected to be less sensitive to hadronization than $\left\langle E_{T}\right\rangle$ and should give a more definitive indication of BFKL behaviour. Mueller 376 suggested that studying deep inelastic events at small $x$, with a measured jet with transverse momentum $k_{T j}^{2} \sim Q^{2}$ and large longitudinal momentum $x_{j}$, such that $z=x / x_{j}$ is small (see Fig. 63a, where the forward jet is indicated as particle $a$ ). The choice $k_{T j}^{2} \sim Q^{2}$ suppresses con- 


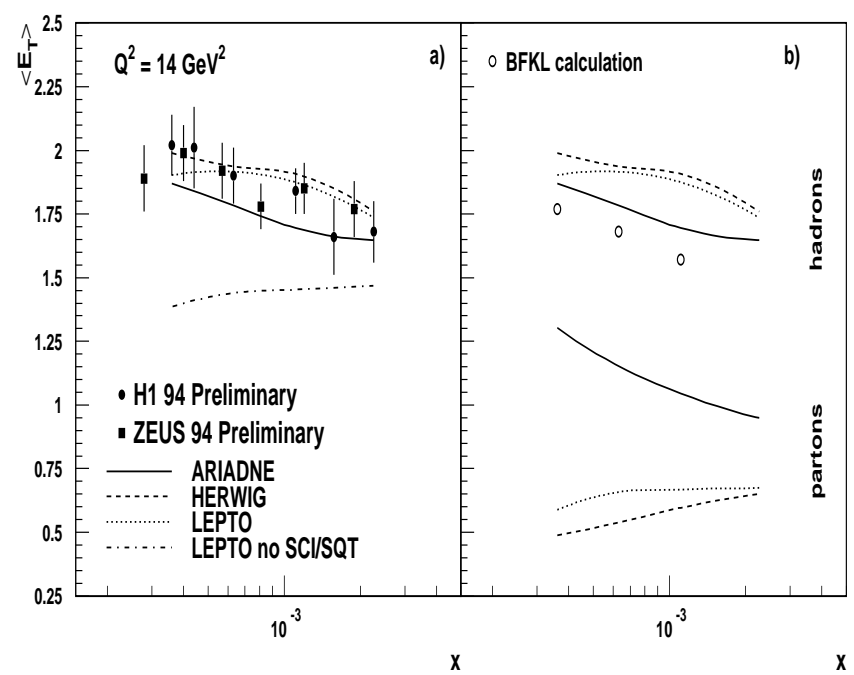

Fig. 65. Mean transverse energy in the central rapidity region at fixed $Q^{2}$ as functions of $x$; (a) shows H1(94) and ZEUS(94) preliminary data compared to the ARIADNE, HERWIG and LEPTO model; (b) Compares hadron and parton level calculations of these models with a BFKL calculation at the parton level.

ventional gluon radiation from DGLAP evolution, since there is no room for such evolution if $k_{T}$ is strongly ordered, whereas BFKL dynamics predicts a cross-section which depends on $z^{-\lambda}$ at small $z$. Hence the cross-section for such events should be much larger for BFKL than for DGLAP dynamics. Moreover the choice $k_{T j}^{2} \sim Q^{2}$ also minimizes the problems of drift into the infra-red region which beset the BFKL calculation for $F_{2}$ (provided $Q^{2}$ is chosen large enough) and the parton distributions need only be used at the large value $x_{j}$, where they are well known ${ }^{\text {dd }}$. Hence the BFKL calculations should be reliable. Such calculations have been made by various authors $358,375,361$.

H1367 377 have produced data on this process from both the ' 93 and '94 runs. We discuss both sets of data, since the '94 data are still preliminary. For the '93 data, DIS events are selected by the cuts, $E_{e}>12 \mathrm{GeV}$, to minimize backgrounds and, $160^{\circ}<\theta_{e}<173^{\circ}$, to concentrate the study at small $x$. These cuts allow a study of forward jet production in the region $Q^{2} \simeq 20 \mathrm{GeV}^{2}$, and $2 \times 10^{-4}<x<2 \times 10^{-3}$. A further cut, $y>0.1$, is necessary to ensure separation of the forward jet from the current jet at the top of the ladder. The jets are found by a cone algorithm, requiring $E_{T}>5 \mathrm{GeV}$ in a cone of radius $R=\sqrt{\Delta \eta^{2}+\Delta \phi^{2}}=1.0$, in pseudorapidity, $\eta$, and azimuthal angle, $\phi$, in the HERA frame. Jets are defined as forward jets if, $x_{j}=E_{j} / E_{p}>0.025,0.5<p_{T j}^{2} / Q^{2}<4,6^{\circ}<\theta_{j}<20^{\circ}$, and $p_{T j}>5 \mathrm{GeV}$ (where

\footnotetext{
dd Note that the parton emerging from the proton is considered to be collinear with the proton and that it emits a soft $t$ channel gluon. Thus the energy of the forward jet is nearly equal to that of the parton emerging from the proton so that $x_{j}$ can be taken to be equal to the longitudinal momentum fraction of this emergent parton.
} 


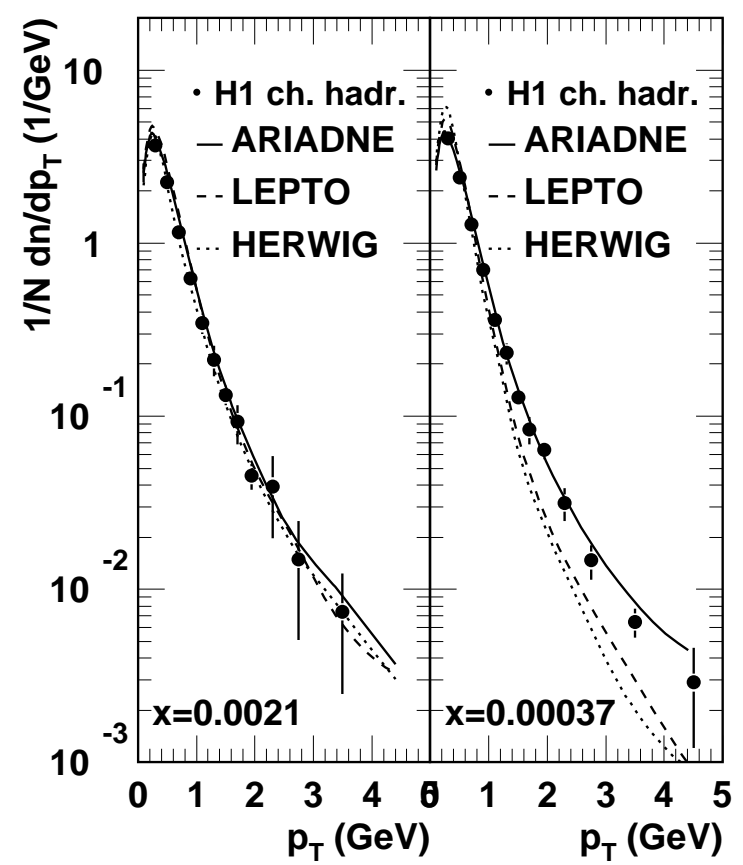

Fig. 66. The $p_{T}$ spectra of the charged particles from $0.5<\eta<1.5$ (CMS). H1(94) data are shown for two different $x$ bins at $Q^{2}=14 \mathrm{GeV}^{2}$. Overlaid are the models ARIADNE, LEPTO and HERWIG (see text).

$p_{T j}$ is taken as an approximation to $k_{T j}$ ). These criteria ensure that $x / x_{j} \lesssim 0.1$. For the 94 data the criteria are modified as follows. The DIS selection cuts remain almost the same, but the $x$ region is shifted to $5 \times 10^{-4}<x<3.5 \times 10^{-3}$. Jets are found by requiring $E_{T}>3.5 \mathrm{GeV}$ in the cone of radius $R=1$, and the criteria to select forward jets are tightened to: $x_{j}>0.035,0.5<p_{T j}^{2} / Q^{2}<2,7^{\circ}<\theta_{j}<20^{\circ}$ and $p_{T j}>3.5 \mathrm{GeV}$.

ZEUS have produced data on forward jets from their ' 95 run 378 with similar DIS selection cuts: $E_{e}>10 \mathrm{GeV}, y>0.1$ : and jet selection cuts: $x_{j}>0.036$, $0.5<p_{T j}^{2} / Q^{2}<2, \theta_{j}>8.5^{\circ}, p_{T j}>5 \mathrm{GeV}$, using the cone algorithm with $R=1$ : and a somewhat wider kinematic range: $Q^{2} \gtrsim 15 \mathrm{GeV}^{2}, 4.5 \times 10^{-4}<x<4.5 \times 10^{-2}$.

This is the best that can currently be done in fulfilling the requirements $k_{T j}^{2} \simeq Q^{2}$ and $x / x_{j}$ small, given the limitations of statistics and the need to separate the forward jet from the proton remnant ${ }^{\mathrm{ee}}$. This requires $x_{j} \lesssim 0.1$, essentially imposed by the lower angular cuts.

For the H1(93) data the number of forward jet events, in two $x$ bins, is shown in Fig. 68 and is compared with the two Monte Carlo models LEPTO and ARIADNE in Table 10. We see that the data favour ARIADNE (where there is no $k_{T}$ ordering) even when the latest versions of each Monte Carlo are used.

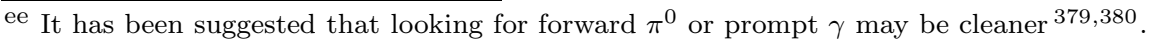




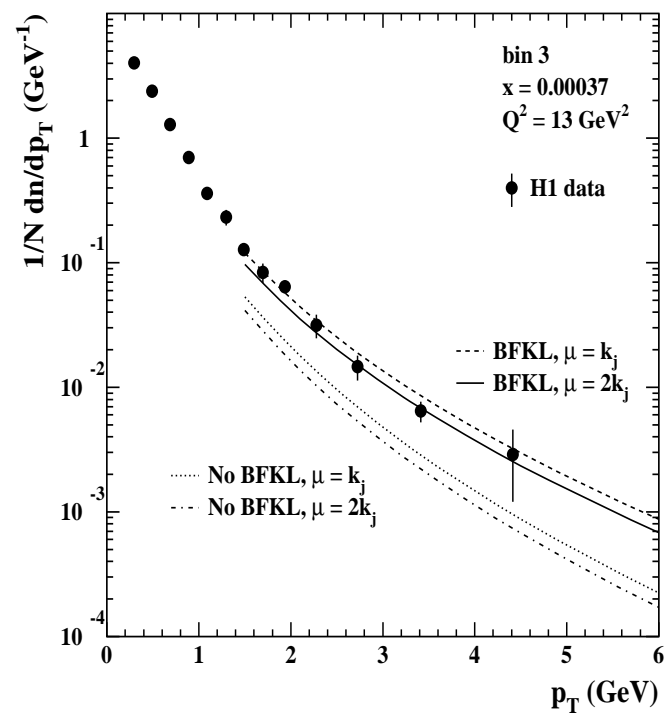

Fig. 67. Transverse momentum distribution (H1(94) data) compared with a calculation (including fragmentation) including and excluding BFKL effects, shown for 2 choices of the scale.

Table 10. Number of observed DIS events with a selected forward jet (H1(93) selection), compared with predictions from the LEPTO(MEPS) and the ARIADNE(CDM) Monte Carlos.

\begin{tabular}{cccc}
\hline$x \times 10^{3}$ & Observed events & MEPS & CDM \\
\hline $0.2 \rightarrow 1.0$ & 271 & 135 & 240 \\
$1.0 \rightarrow 2.0$ & 158 & 96 & 121 \\
\hline
\end{tabular}

The H1(94) data have sufficient statistics to look at the cross-section as a function of $x$ in six $x$ bins. They are shown in Fig. 69a together with the predictions of the LEPTO and ARIADNE Monte Carlos. ARIADNE gives a reasonable description of the data, while LEPTO is somewhat worse. Furthermore, Fig. 69b shows that for the jets selected it appears that the hadronization corrections are again large for the LEPTO model, somewhat masking the true parton dynamics. These effects should become smaller for increased jet $E_{T}$. However, it has been shown 381 that, in the kinematic range considered, increasing $E_{T}$ reduces the sensitivity to the BFKL effect.

The ZEUS '95 data is shown in Fig. 70. The wider kinematic range allows us to see clearly that the Monte Carlos agree with each other and with the data at higher $x$ but begin to disagree for $x<10^{-2}$. ARIADNE gives a successful description of the data. However, as remarked earlier, we should not interpret the success of ARIADNE as a definitive indication for BFKL dynamics because the ARIADNE Monte Carlo does not actually incorporate BFKL evolution. Moreover, the current LEPTO/HERWIG and ARIADNE Monte Carlos do not treat the conventional dy- 


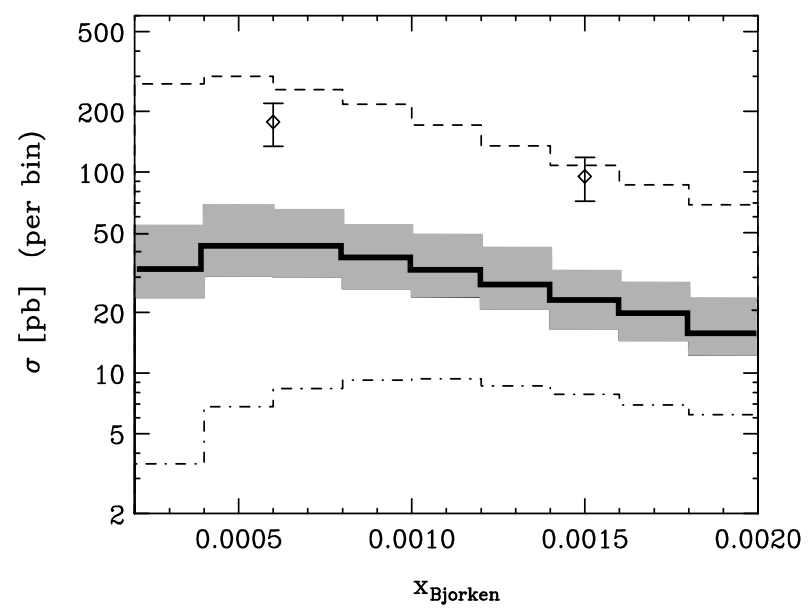

Fig. 68. Forward jet cross-section as a function of $x$ from the NLO(LO) MEPJET calculations given as the solid(dash-dot) histograms. The BFKL calculation of Bartels is shown as the dashed histogram. H1(93) data are shown for comparison

namics of jet production exactly beyond LO. We need calculations of both BFKL and conventional dynamics to higher orders, in order to make a fair comparison. Such calculations are just becoming available.

There are now three different Monte Carlos incorporating conventional NLO jet calculations, MEPJET by Mirkes and Zeppenfeld 382, DISENT by Catani and Seymour 383 and DISASTER ++ by Graudenz 384 . Results indicate that the conventional predictions for the jet cross-sections with one of the jets going forward, with large $x_{j}$ and $k_{T j}^{2} \sim Q^{2}$, are very sensitive to the inclusion of higher orders. When a forward jet is required the NLO $(2+1)$ jet cross-section exceeds the LO $(2+1)$ jet cross-section by a factor of $\sim 4$, and further corrections may be expected from higher order effects. However these are not expected to be so dramatic, since the main effect of the LO to NLO correction is dominated by the special kinematic requirements of these jets. The NLO calculation of the forward jet cross-section from MEPJET is shown compared to the H1(93) data in Fig. 68 and compared to the ZEUS 95 data in Fig. 70. The NLO calculation from DISENT is shown in Fig. 69b. These calculations indicate that conventional dynamics at NLO is still inadequate to explain the data.

Bartels et al 361 have compared a BFKL calculation with an analytic calculation of conventional dynamics at the Born level. For the BFKL calculations one considers the cross-sections for the processes $g \rightarrow g+(n g)+q \bar{q}$ and $q \rightarrow q+(n g)+q \bar{q}$, where the many gluons, $(n g)$, are summed by the BFKL ladder. For the analytic calculation one considers the Born cross-sections for these processes without $(n g)$. The calculations are subject to the experimental cuts used by the HERA collaborations, where both the '93 and the '94 data are considered, but hadronization of the forward parton into a jet is not considered. These calculations are illustrated on the H1(93) data in Fig. 68, on the H1(94) data in Fig. 69b and on the ZEUS 


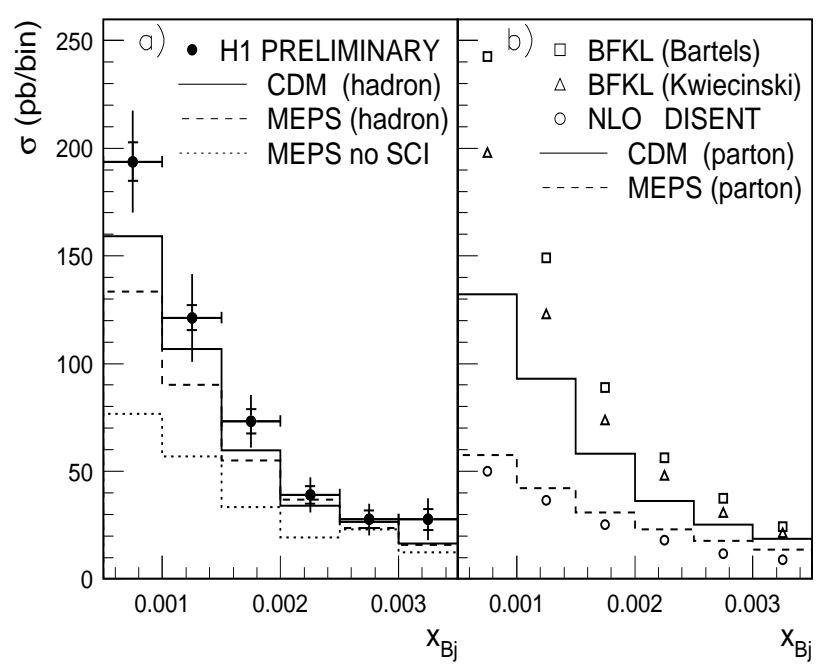

Fig. 69. a) H1(94) preliminary data on the forward jet cross-section as a function of $x$ compared to the hadron level predictions of the ARIADNE(CDM) and LEPTO(MEPS) Monte Carlos (both with and without SCI): b) parton level predictions from the same Mente Carlos, and from NLO Monte Carlo DISENT and the BFKL calculations of Bartels et al 361 and Kwiecinski et al 375

'95 data in Fig. 70. Kwiecinski et al 375 have also made a BFKL calculation of the forward jet cross-section at the parton level, which differs from that of Bartels et al by including massive charm contributions and by incorporating the running of $\alpha_{s}$ numerically. This calculation is compared to the H1(94) data in Fig. 69b.

These comparisons seem to indicate that BFKL dynamics are necessary. However, we should be cautious because there are still some limitations to the calculations. It has been found that conventional NLO calculations also cannot explain the rate of centrally produced dijets in HERA data212. However, a study of NLO calculations for jet production in photoproduction events has shown that the calculation 213 become unreliable in some special regions of phase space for dijet quantities defined with equal cuts on the transverse energy of the observed jets, as is usually done for dijet measurements. This issue needs to be clarified for the DIS case. The discrepancy between data and conventional NLO calculations could also be due to the need for conventional higher order corrections or it may be attributable to more subtle BFKL effects. The BFKL calculations are also not exact: charm production is not properly treated and hadronization is still not included. We also note that Jung has suggested that a resolved photon contribution in DIS eyents could be a possible explanation for rising forward jet cross-sections at low $x 385$.

In conclusion the data from the hadron final states gives tantalizing indications that BFKL dynamics are necessary. We await further developments both theoretical, in more sophisticated calculations, using the newly developed BFKL and CCFM(LDC) Monte Carlos, and experimental, in measurement of further interest- 

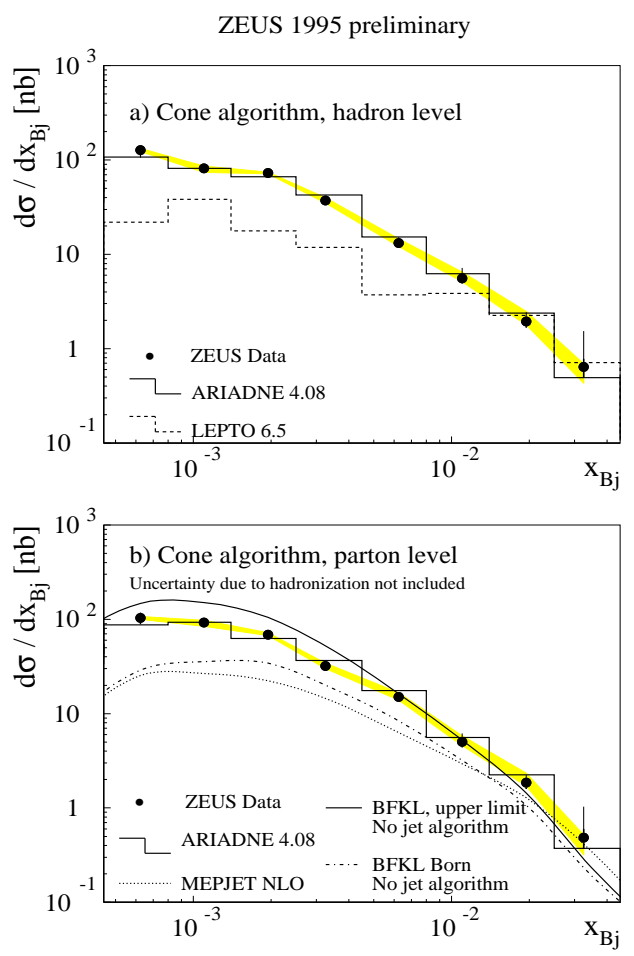

Fig. 70. ZEUS 95 preliminary data on the forward jet differential cross-section as a function of $x$ compared to the hadron level and parton level predictions of the ARIADNE and LEPTO nte Carlos and to the NLO Monte Carlo MEPJET and the BFKL calculations of Bartels et al 361

ing processes. We note that a promising novel way to investigate BFKL dynamics has been proposed for deep inelastic $\gamma^{*} \gamma^{*}$ scattering at LEP and at future linear $e^{+} e^{-}$colliders 386 . 387 . The total cross section for this process is expected to be strongly affected by BFKL dynamics. The 1997 LEP data may yield a first measurement of this process.

\section{The low $Q^{2}$ region}

In Sec. 5 it was shown that the HERA data now cover the region down to $Q^{2} \sim 0.1 \mathrm{GeV}^{2}$. The major goal for these data is the study of the transition from deep inelastic scattering to the photoproduction $\left(Q^{2} \rightarrow 0\right)$ limit. At $Q^{2}=0$ the dominant processes are of non-perturbative nature and are well described by Regge phenomenology. As $Q^{2}$ increases, the exchanged photon is expected to shrink and pQCD to take over. The HERA data clearly demonstrate that the inclusive crosssections in the region $Q^{2}>1 \mathrm{GeV}^{2}$ can be described by pQCD. How far down in $Q^{2}$ does pQCD continue to work? Where exactly is the transition region and is this transition smooth? These are some of the intriguing questions to be answered by these low $Q^{2}$ data. The transition region is expected to shed light on the interplay 
between soft and hard interactions. Several phenomenological models attempt to describe this region and will be compared to the data.

It is instructive to remember that the behaviour of $F_{2}$ at small $x$ is related to the behaviour of $\sigma^{\gamma^{*} p}$ at large $\gamma^{*} p$ centre of mass energy $W$. Recall Eqs. $122-124$ and the small $x$ approximation Eq. 126. For ease of reference we shall write this as

$$
\sigma^{\gamma^{*} p}\left(W^{2}, Q^{2}\right) \approx \frac{4 \pi^{2} \alpha}{Q^{2}} F_{2}\left(x, Q^{2}\right)
$$

where $\sigma^{\gamma^{*} p}=\sigma_{T}+\sigma_{L}$. As we discussed in Sec. 3.2, Regge theory provides a reasonable description of the $s$ dependence of the photoproduction cross-section, and we might thus expect it to describe low $Q^{2}$ DIS data. We recall that in this picture the $s\left(\gamma^{*} p\right)=W^{2}$ dependence of the cross-section at high energy is given by $\sigma^{\gamma^{*} p} \sim W^{2 \lambda}$, where $\lambda$ is a constant determined by the intercept of the soft Pomeron trajectory $\alpha_{P}=1+\lambda$, and $\lambda \simeq 0.08$ gives a good fit to hadron-hadron crosssections 17. (We note that Cudell et al 388 have suggested that $\lambda=0.096$ gives a better fit.) Using this idea Donnachie and Landshoff (DOLA) 389 developed a model which successfully described the low $Q^{2}$ fixed target $F_{2}$ data (for $Q^{2}<10 \mathrm{GeV}^{2}$ ) and gave the correct limit as $Q^{2} \rightarrow 0$. The structure function is described by a sum of two terms: one for the Pomeron (which describes the contribution of sea quarks and dominates at high energy) and one for a Reggeon of intercept $\alpha_{R} \simeq 0.5$ (which describes the contribution of valence quarks). Each of these terms takes the form

$$
\left(\frac{Q^{2}}{Q^{2}+M_{R, P}^{2}}\right)^{\alpha_{R, P}} x^{\left(1-\alpha_{R, P}\right)}(1-x)^{\beta_{R, P}}
$$

By construction the $W$ dependence of $\sigma^{\gamma^{*} p}$ is very weak, as it is for hadron-hadron total cross-sections. At HERA it is clear that this simple picture cannot be adequate as the steep rise of $F_{2}$ as $x \rightarrow 0$ becomes a strong rise of $\sigma^{\gamma^{*} p}$ as $s$ increases. This is shown in Fig. 71 which shows $\sigma^{\gamma^{*} p}$ as a function of $W^{2}$ for different values of $Q^{2}$ from 0 to $2000 \mathrm{GeV}^{2}$.

It is of interest to check if the new low $Q^{2}$ data from HERA approach the DOLA limit. Data in the $Q^{2}$ range, $0.11<Q^{2}<6.5 \mathrm{GeV}^{2}$, are shown in Fig. 72 compared with the DOLA and GRV94 (see Sec. 6.1.5) predictions. Considering the HERA data together with the lower $W^{2}$ data from E665, we see that the energy dependence predicted by the DOLA model is too weak for $Q^{2}$ values above $\sim 0.4 \mathrm{GeV}^{2}$. For lower $Q^{2}$ values we do not have the benefit of a large $W$ lever arm in the data. The shape of the model is compatible with the data in this limited $W$ interval, but the magnitude of the DOLA prediction lies significantly below the data. We note that the HERA photoproduction data were included in the DOLA fit and hence affect the normalization somewhat These photoproduction cross-sections have total errors of typically $15 \%-20 \% 390$. 391 . By contrast we see that the GRV prediction describes the data reasonably well for higher $Q^{2}$ and begins to fail for $Q^{2} \lesssim 0.8 \mathrm{GeV}^{2}$ (as shown more clearly in Fig. 74). ZEUS has studied the effect of lowering the starting scale $Q_{0}^{2}$ and including lower $Q^{2}$ data in the NLO DGLAP fit which they used to extract the gluon distribution (see Sec. 6.1.3). The result is shown in Fig. 73. It is found that standard DGLAP works well for data and starting 


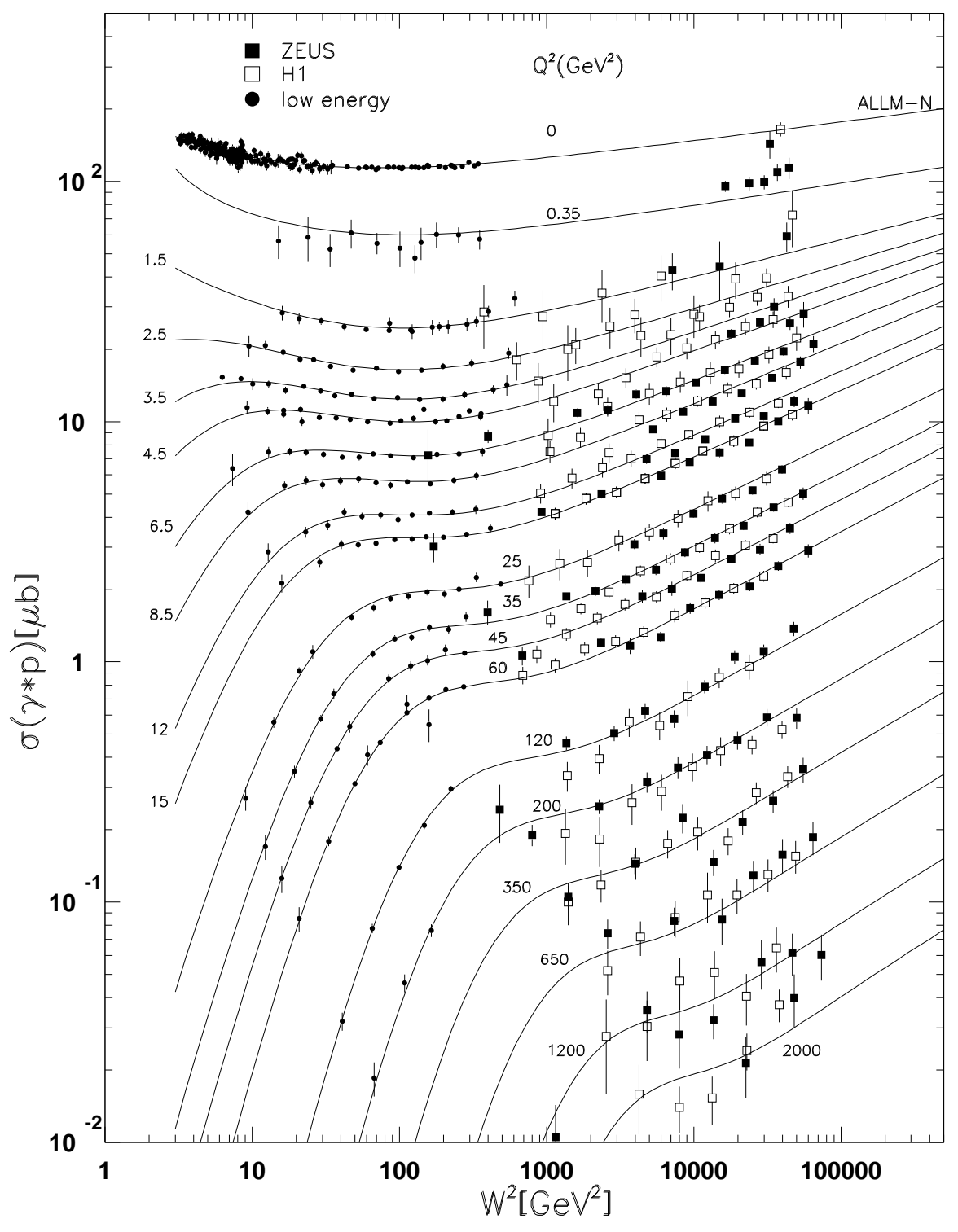

Fig. 71. The total $\gamma^{*} p$ cross-section $F_{2}$ measurements as a function of $W^{2}$ for different $Q^{2}$. The curves are the model of ALLM 394. 
scales $Q_{0}^{2}$ as low as $0.8 \mathrm{GeV}^{2}$, but for fits starting from lower $Q^{2}$ values the scaling violations become too large at low $x$. ZEUS has also introduced higher twist terms of the form $f(x) / Q^{2}$ into this fit and it is found that such terms are negligible for $x<0.02$ and that the fit it still unable to describe the data for starting scales lower than $0.8 \mathrm{GeV}^{2}$. This comparison demonstrates that data with $Q^{2}$ less than about $0.8 \mathrm{GeV}^{2}$ approach the pure soft regime, and can no longer be described by leading twist pQCD (DGLAP) calculation, or even by the inclusion of conventional higher twist terms. Hence these data constitute the effective transition region, and the transition from DIS to photoproduction appears to be smooth.

We now turn to the phenomenological developments and models which aim to describe this region. More details and the formulae for many of the models discussed below are summarized in ref. 392 and in the very extensive review of low $x$, low $Q^{2}$ electroproduction by Badelek and Kwiecinski 393 . Comparisons with data are presented in Fig. 74, where $F_{2}$ is shown as a function of $x$ for the smallest $Q^{2}$ bins, and in Fig. 75, where the quantity $\sigma^{\text {eff }}$ is shown as function of $Q^{2}$ for different $W$ bins. The quantity $\sigma^{e f f}$ is the effective measured virtual photon-proton crosssection, $\sigma^{e f f}=\sigma_{T}+\varepsilon \sigma_{L}$, for ep collisions in the HERA kinematic range, as defined by Eqs. 122, 124, 125. It is used because it can be determined from the data without assumptions about $R$. Since $\varepsilon \gtrsim 0.9$, for most of the kinematic range, it also represents most of the total $\sigma^{\gamma^{*} p}$ cross-section.

A number of groups have modified the Regge approach of DOLA by allowing the parameter $\lambda\left(=\alpha_{P}-1\right)$, which fixes the Pomeron intercept, to vary with $Q^{2}$, for example ALLM 394 and CKMT 395. Supposing that the soft Pomeron of DOLA, is not a 'bare' Pomeron but a shadowed Pomeron, then as $Q^{2}$ increases shadowing gradually disappears and the bare Pomeron is revealed to be a harder Pomeron of larger intercept 395] 396. In the approach of CKMT the data are fitted to the sum of contributions from Pomeron exchange and from Reggeon exchange using similar forms to that given in Eq. 196. However, the intercept of the Pomeron Regge trajectory is expressed as $\alpha_{P}=1+\lambda\left(Q^{2}\right)$ and the form taken for the $Q^{2}$ dependence is $\lambda\left(Q^{2}\right)=\lambda_{0}\left(1+2 Q^{2} /\left(Q^{2}+d\right)\right)$, where the parameters $\lambda_{0}$ and $d$ have been determined to be $\lambda_{0}=0.07684$ and $d=1.117 \mathrm{GeV}^{2}$, from a fit to $\operatorname{NMC}(92)$ data in the region $1<Q^{2}<5 \mathrm{GeV}^{2}$ and real photoproduction data from HERA and from lower $W$ fixed target experiments. The model assumes that this prescription accounts for non-perturbative contributions to $F_{2}$ for $Q^{2}$ values up to about $2 \mathrm{GeV}^{2}$. For higher $Q^{2}$ the pQCD DGLAP evolution equations are applied to predict the $Q^{2}$ dependence of $F_{2}$. Thus, since the effective value of $\lambda$ quickly becomes larger than that used by DOLA as $Q^{2}$ increases, the structure function $F_{2}$ predicted by CKMT rises faster with decreasing $x$ (as $Q^{2}$ increases) than the DOLA prediction, as shown in Fig. 74. However, although the CKMT prediction is systematically above that from DOLA, for $Q^{2} \gtrsim 0.3 \mathrm{GeV}^{2}$ it is still below the data.

The model of Abramowicz et al 394 (ALLM) is also based on a Regge motivated approach extended into the large $Q^{2}$ regime compatibly with pQCD expectations. Contributions from Reggeon and Pomeron exchange, of a similar form to that given 


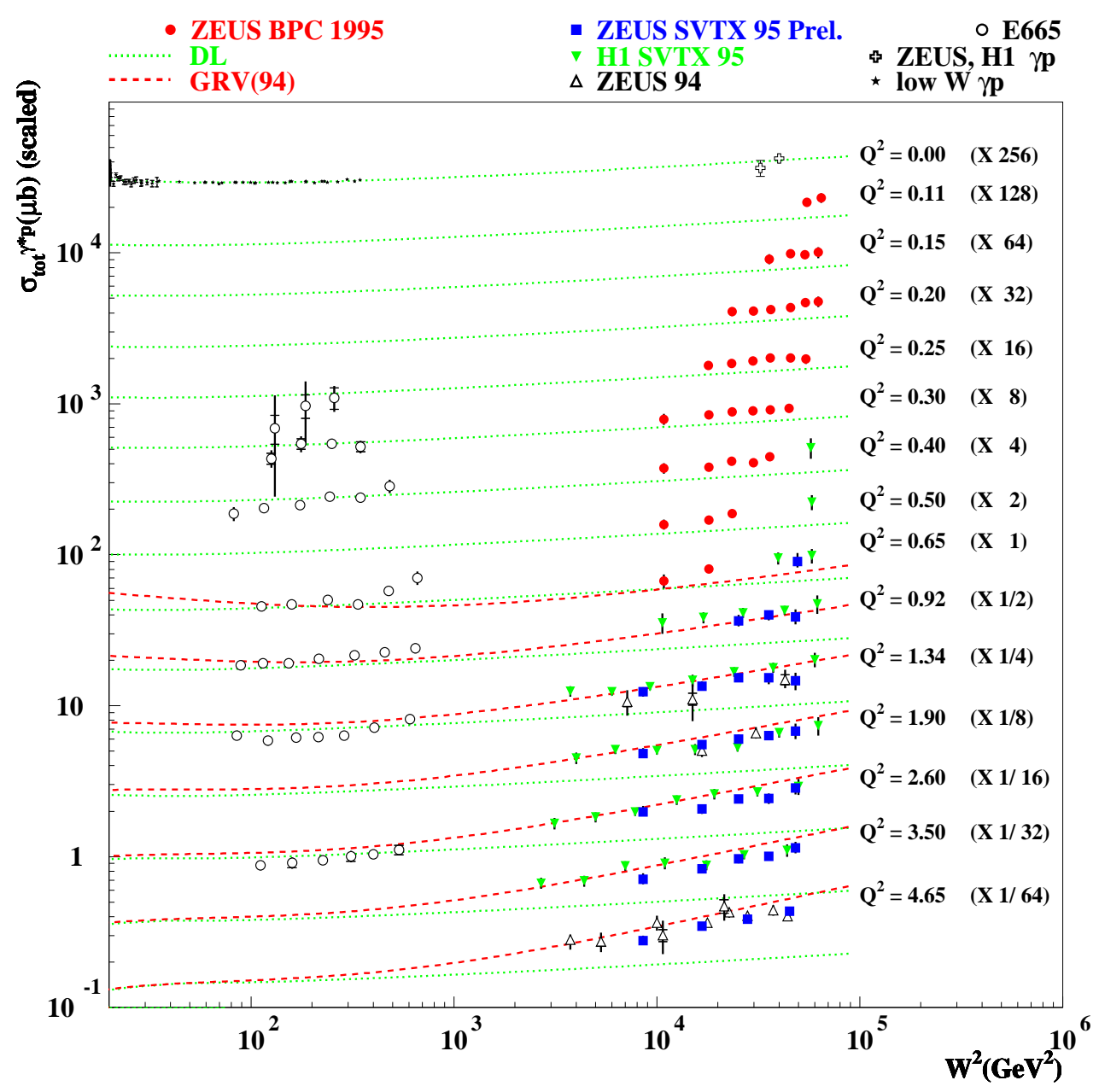

Fig. 72. The total $\gamma^{*} p$ cross-section from $F_{2}$ measurements as a function of $W^{2}$ at low $Q^{2}$. The curves are the models of DOLA (dotted lines) and GRV (dashed lines). 


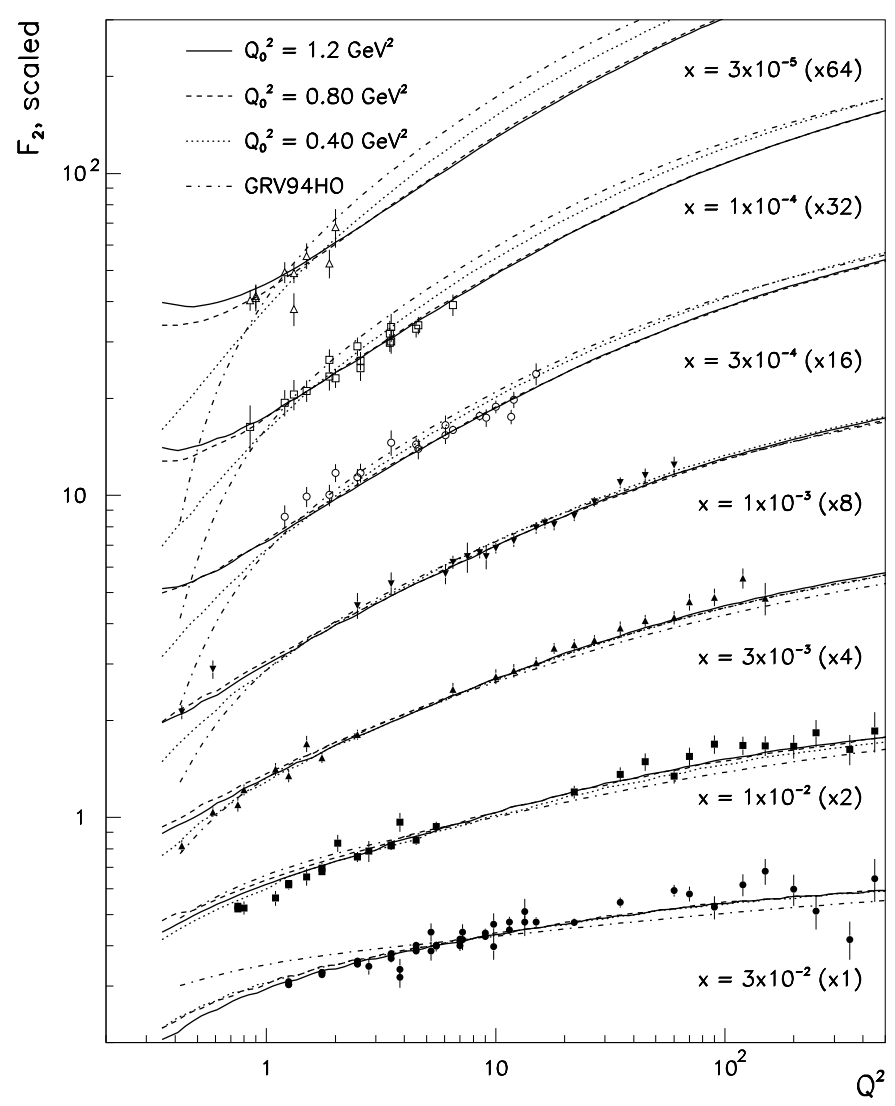

Fig. 73. $F_{2}$ data and results from a perturbative QCD DGLAP global fit for 3 different starting scales $Q_{0}^{2}=0.4,0.8$ and $12 . \mathrm{GeV}^{2}$. The GRV prarametrization for $F_{2}$ is shown for comparison. The data sets used are ZEUS(94), ZEUS(95)SVX (preliminary) and H1(95)SVX, ZEUS(95)BPC, NMC(97), BCDMS, SLAC, E665. The data in each $x$ bin are scaled by the factors indicated in brackets

in Eq. 196, are generalized such that the dependence $x^{1-\alpha_{R, P}}$ becomes $x^{-\lambda_{R, P}\left(Q^{2}\right)}$ for both the Reggeon and the Pomeron terms and the parameters $\lambda_{R, P}$ vary with $Q^{2}$ logarithmically, emulating pQCD evolution in the high $Q^{2}$ region. This model gives a parametrization of the whole $x, Q^{2}$ phase space. It was fitted to DIS and photoproduction data before the final HERA(94) and HERA(95)(low $\left.Q^{2}\right)$ data became available. The fitted value of $\lambda_{P}$ changes smoothly from 0.08 at $Q^{2}=0$, to about 0.4 at $Q^{2} \sim 10^{3} \mathrm{GeV}$. In Fig. 75 one can see that this model has the correct limit at $Q^{2}=0$ and that it agrees with data for $Q^{2}>2 \mathrm{GeV}^{2}$. However the prediction is below the data for small $Q^{2}$ values, such that the transition from the soft to the hard regime is too slow.

The model of Badelek and Kwiecinski 397 (BK) combines the concepts of Generalized Vector Meson Dominance (GVMD) with dynamical parton models such as that of GRV. Here the structure function is assumed to be the sum of two contribu- 


\section{ZEUS 1995}

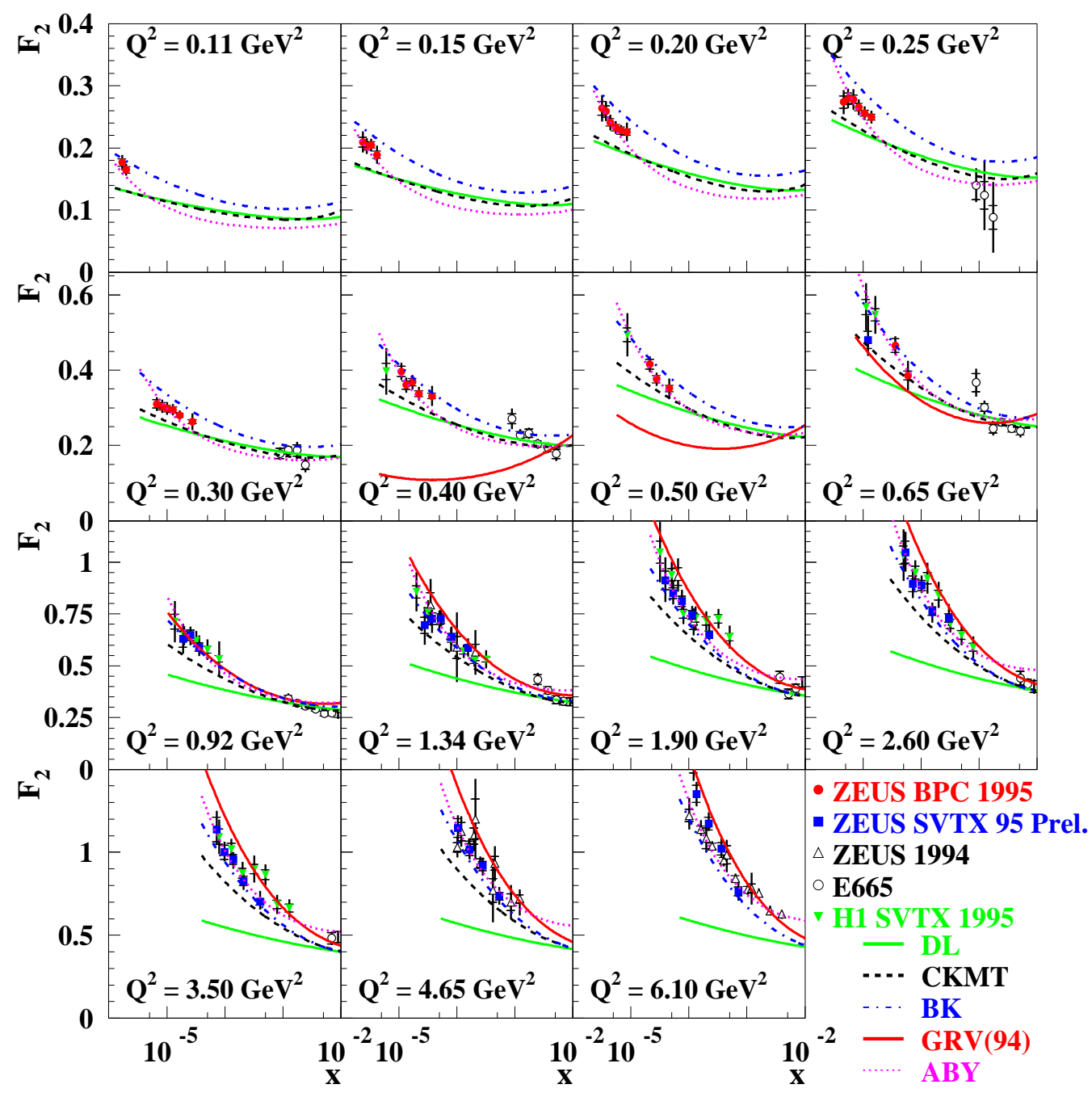

Fig. 74. Measurement of the proton structure function $F_{2}\left(x, Q^{2}\right)$ in the low $Q^{2}$ region by H1(95)SVX (full triangles), ZEUS(95)BPC (full circles) and ZEUS(95)SVX (preliminary)(full squares) together with previously published results from ZEUS(94) (open triangles) and E665 (open squares). Various predictions for $F_{2}$ are compared with the data: the model of DOLA (full line/small), the model of CKMT (dashed line ), the model of BK (dashed-dotted line), the parametrization of GRV (full line/large) and the model of ABY (dotted line). 


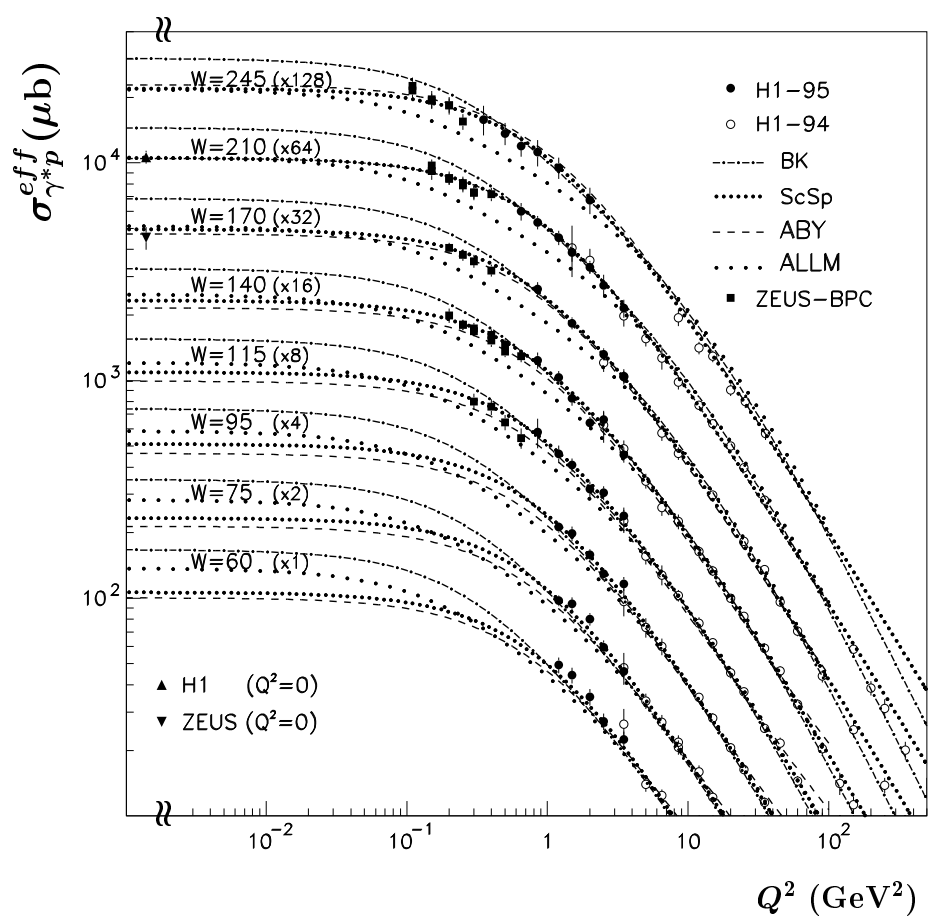

Fig. 75. Measurement of the virtual photon-proton cross-section $\sigma_{\gamma^{*} p}^{e f f}$ as a function of $Q^{2}$ at various values of $W$ (in GeV), from H1(95)SVX and H1(94) (circles) and ZEUS(95)BPC (squares). The cross-sections for consecutive $W$ values are multiplied with the factors indicated in brackets. The errors represent the statistical and systematic errors added in phe photoproduction points (cross: $W=210 \mathrm{GeV}$, diamond: $W=170 \mathrm{GeV}$ ) are from 390.391. Global normalization uncertainties are not included in the errors shown. The curves represent the ALLM (dotted/small), BK (dashed-dotted line), ScSp (dotted line/large) and ABY (dashed)

tions: a GVMD model term $F_{2}^{V M D}$ and a partonic term $F_{2}^{\text {part }}$. For the latter the GRV model was used and it becomes dominant above $Q^{2} \sim 1 \mathrm{GeV}^{2}$. In this model $F_{2}$ is given by

$$
F_{2}\left(x, Q^{2}\right)=F_{2}^{V M D}\left(x, Q^{2}\right)+\frac{Q^{2}}{Q^{2}+Q_{V M D}^{2}} F_{2}^{\text {part }}\left(\bar{x}, Q^{2}+Q_{V M D}^{2}\right)
$$

with $\bar{x}=\left(Q^{2}+Q_{V M D}^{2}\right) /\left(W^{2}+Q^{2}+Q_{V M D}^{2}\right)$. Vector mesons with mass squared smaller than $Q_{V M D}^{2}$ are included in the $F_{2}^{V M D}$ term. The value of $Q_{V M D}^{2}$ is the only parameter of this model and it was chosen, rather than fitted, to be $1.2 \mathrm{GeV}^{2}$ before the HERA low $Q^{2}$ data were available. The model has perforce a smooth transition from the pQCD region to the real photon limit, but the predictions lie above the data in the limit $Q^{2} \rightarrow 0$, as can be seen in Fig. 75. This figure and Fig. 84 illustrate that there is better agreement with HERA data for the BK model 
than for the ALLM, however there is a tendency for the BK predictions to lie too high at the lowest $Q^{2}$ values, whereas the ALLM predictions lie too low. Thus it seems that the transition from the hard to the soft regime is also too slow in the BK parametrization.

A GVMD inspired approach has been proposed by Schildknecht and Spiesberger (ScSp) 398 to fit the low and medium $Q^{2}$ HERA data up to $Q^{2}$ values of $350 \mathrm{GeV}^{2}$. For real photoproduction the cross-section is almost saturated (up to $78 \% 399$ ) by low mass vector meson states, namely $\rho^{0}, \omega, \phi$. For non-zero $Q^{2}$ these contributions decrease rapidly and, according to GVMD, their role at small $x$ is taken over by more massive states. The authors make a 4 parameter fit to all available $\gamma^{*} p$ data with $W>30 \mathrm{GeV}$, and obtain a remarkably good description of the HERA data, as shown in Fig. 75. A similar approach has been proposed in 400 , where in addition to the pure GVMD contribution, a region of large masses, $m$, and large coherence lengths, $l=\left(M x\left(1+m^{2} / Q^{2}\right)\right)^{-1}$, of photon fluctuations is considered. In this region the authors expect that the hadronic behaviour of the photon becomes a complicated multi-jet state. This model allows for good fits to HERA(94) data and fixed target data, but has not been confronted yet with the very low $Q^{2} \operatorname{HERA}(95)$ data.

A different approach to the low $Q^{2}$ behaviour of $F_{2}$ in the transition region has been presented in 311,13 by Adel, Barreiro and Yndurain (ABY). It assumes that perturbative QCD evolution is applicable to the lowest values of $Q^{2}$. The strong coupling constant is assumed to become independent of $Q^{2}$ for values below roughly $1 \mathrm{GeV}^{2}$, that is $\alpha_{s}$ 'saturates'. There are assumed to be two contributions to $F_{2}$, one with a singular and one with a non-singular input (see Sec. 7.2.2) to pQCD evolution. These translate into hard and soft contributions to the $\gamma^{*} p$ cross-section respectively. The hard contribution prevents $F_{2}$ decreasing with decreasing $x$ for $Q^{2}$ values below $1 \mathrm{GeV}^{2}$ and thus remedies the failure of the GRV approach. The form of the singular input is $\sim x^{-\lambda_{S}}$, with $\lambda_{S}=0.47$ independent of $Q^{2}$, chosen to ensure that $F_{2} \rightarrow 0$ as $Q^{2} \rightarrow 0$. The form of the non-singular input is $\sim Q^{2} /\left(Q^{2}+M^{2}\right)$, $M=0.87 \mathrm{GeV}$, and this does not evolve with $Q^{2}$ for $Q^{2} \lesssim 10 \mathrm{GeV}^{2}$. Above this $Q^{2}$ a constant term is used for the non-singular input to the DGLAP equations, and evolution is started from $Q_{0}^{2} \sim 2 \mathrm{GeV}^{2}$. The result of a fit of the ABY approach to data is compared with the new $F_{2}$ results in Fig. 74 and Fig. 75 . Note that this fit used the HERA(94) data $\left(Q^{2}>1.5 \mathrm{GeV}^{2}\right)$ and also a preliminary version of the ZEUS(95)BPC low $Q^{2}$ data, but not the H1(95)SVX low $Q^{2}$ data. There is good agreement with present data, but one can see that in this approach the rise of $F_{2}$ with decreasing $x$ occurs at lower $x$ than for any of the other approaches, namely for $x<10^{-4}$. In this sort of approach the singular (hard) term always dominates as $x \rightarrow 0$ ( $W$ increases). Thus it should be easy to check these predictions with lower $x$ data.

There are several other models which use both a hard and a soft component to explain the data in the transition region 401402403 . Gotsman et al 403 have developed an explanation for the transition region using soft and hard contributions, which 
suggests both that the hard contribution can still be significant at $Q^{2}=0$ and that the soft contribution can be sizeable at large $Q^{2}$. Both ABY and Troshin and Tyurin 401 have observed that the Donnachie Landshoff fit of the soft Pomeron to high energy hadron-hadron cross-section data using the form, $\sigma_{t o t} \sim s^{0.08}$, is not the only good fit to available data. The form, $\sigma_{t o t} \sim a+b s^{0.5}$, which combines contributions from a soft and a hard Pomeron, also gives a good fit. One may contrast approaches which consider two Pomerons, hard and soft, with approaches which consider a single Pomeron whose intercept changes with $Q^{2}$. It is important to remember that both of the forms given above are pre-asymptotic, unitarity requires that cross-sections rise as $\ln ^{2} s$ asymptotically ${ }^{\mathrm{ff}}$.

The ABY and GVDM models appear to give a good description of the data shown in Fig. 75. Can we be satisfied with these models? The answer is partially given by checking the $W$ dependence of the photoproduction cross-section 404 . This is shown in Fig. 76 for the ALLM, BK, ABY and GVDM models. The ALLM model includes all these data in their fit, while other models include data above a minimum $W$ value (ScSp, ABY), or no data at all (BK). Consequently ALLM describes the data, while the other models fail. In particular, naively extrapolating the GVDM prediction to low $W$ yields negative cross-sections for $W<6 \mathrm{GeV}$. It has also been pointed out that the ratio $R$ for this model is anomalously high in the high $Q^{2}$ region 405 .

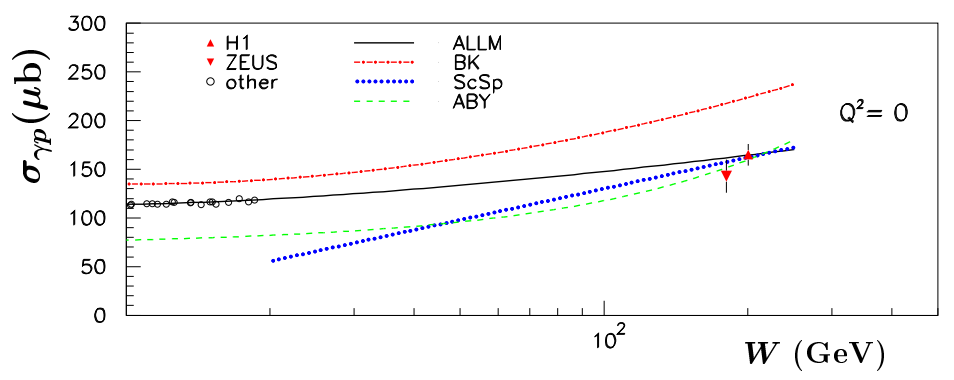

Fig. 76. The $W$ dependence of the photoproduction cross-section. The curves represent the ALLM (full line), BK (dashed-dotted line), ScSp (dotted line/large) and ABY (dotted line/small)

In summary, it turns out that the region $0.1<Q^{2}<1 \mathrm{GeV}^{2}$ spans a kinematic range in which Regge or VMD inspired models describe the data at low $Q^{2}$, and models based on pQCD account well for the higher $Q^{2}$ domain (but see the discussion in Sec. 7.6 for comments on the reliability of conventional pQCD at such $Q^{2}$ values). The road towards a full understanding of this region lies in finding a proper marriage between these two limits, as has already been attempted with partial success, and further work is in progress 405 . Clearly the HERA data in the

\footnotetext{
ff Strictly one should not use the terminology Pomeron in the pre-asymptotic regime, but this has now become common practice.
} 
low $\left(x, Q^{2}\right)$ region will help to discriminate between different theoretical approaches to low- $Q^{2}$ dynamics. Improved measurements of $\sigma^{\gamma p}$ over a wider $W$ range from HERA will also be important.

\section{9. $e p$ cross-sections at very large $Q^{2}$}

Complete formulae for the $\mathrm{NC}$ and $\mathrm{CC}$ cross-sections applicable at high $Q^{2}$ have been given in Secs. 2.2, 2.3. At $Q^{2}$ of order $M_{Z}^{2}$ the parity violating structure function $F_{3}$ gives a positive contribution to $\sigma^{N C}\left(e^{-} p\right)$ but reduces $\sigma^{N C}\left(e^{+} p\right)$. At such large $Q^{2}$ values, the CC cross-section is of comparable magnitude to the $\mathrm{NC}$ - a visible manifestation of electroweak unification - with $\sigma^{C C}\left(e^{-} p\right)$ larger than $\sigma^{C C}\left(e^{+} p\right)$ again from the $F_{3}$ contributions.

Fig. 77 shows the cross-sections at large $Q^{2}$ from HERA data collected in the period 1993-95 406. At $Q^{2} \sim 100 \mathrm{GeV}^{2} \sigma^{N C}$ is dominated by $\gamma$ exchange, is charge independent and is two orders of magnitude larger than the CC cross-section. For $Q^{2} \geq 4000 \mathrm{GeV}^{2}$ the measured $\mathrm{CC}$ and NC cross-sections are indeed of comparable magnitude, with some indication of charge dependence. The precision of the data do not yet permit a measurement of $x F_{3}$.

Apart from providing a foretaste of future physics at HERA, the measurement of high $Q^{2}$ cross-sections has recently been a very topical issue. In February 1997 the DESY Laboratory announced that both H1 and ZEUS had observed an excess of NC events above the expectation of the Standard Model calculation for $Q^{2}>15000 \mathrm{GeV}^{2}$. Detailed results have since been published by $\mathrm{H} 1407$ and ZEUS 408. While it is not our intention to consider this in any detail, we would like to summarise some characteristics of the data, to discuss the reliability of the Standard Model (SM) calculation and to summarise very briefly some of the possible explanations.

\subsection{The Standard Model calculation}

To calculate the Standard Model NC and CC cross-sections at large $Q^{2}$ requires knowledge of the parton distributions at large $x$, for example at $Q^{2}=5000 \mathrm{GeV}^{2}$ the $x$ range is roughly 0.05 to 1 . In this region of $x$ one is relying on the high statistics data from SLAC and BCDMS to determine the $x$ dependence. The valence quarks dominate and their momentum distributions are relatively well determined by the global fits. At the very largest $Q^{2}$ values considered, the $u$ quark contribution is the most important. The large $x$ region generally is also where one has greatest confidence in the DGLAP equations. Higher twist effects can be minimised by suitable cuts on the input data. All the required Standard Model input couplings and masses are very well determined 409 , many of them from the LEP1. The uncertainty on the cross-section comes largely from the systematic uncertainty in the large $x$ input data and from the uncertainty in $\alpha_{s}$. One possible estimate of the uncertainty in the structure functions at large $Q^{2}$ is simply to take recent global fit results, e.g. CTEQ4 and MRSR and compare them. The fitting teams have also considered the 


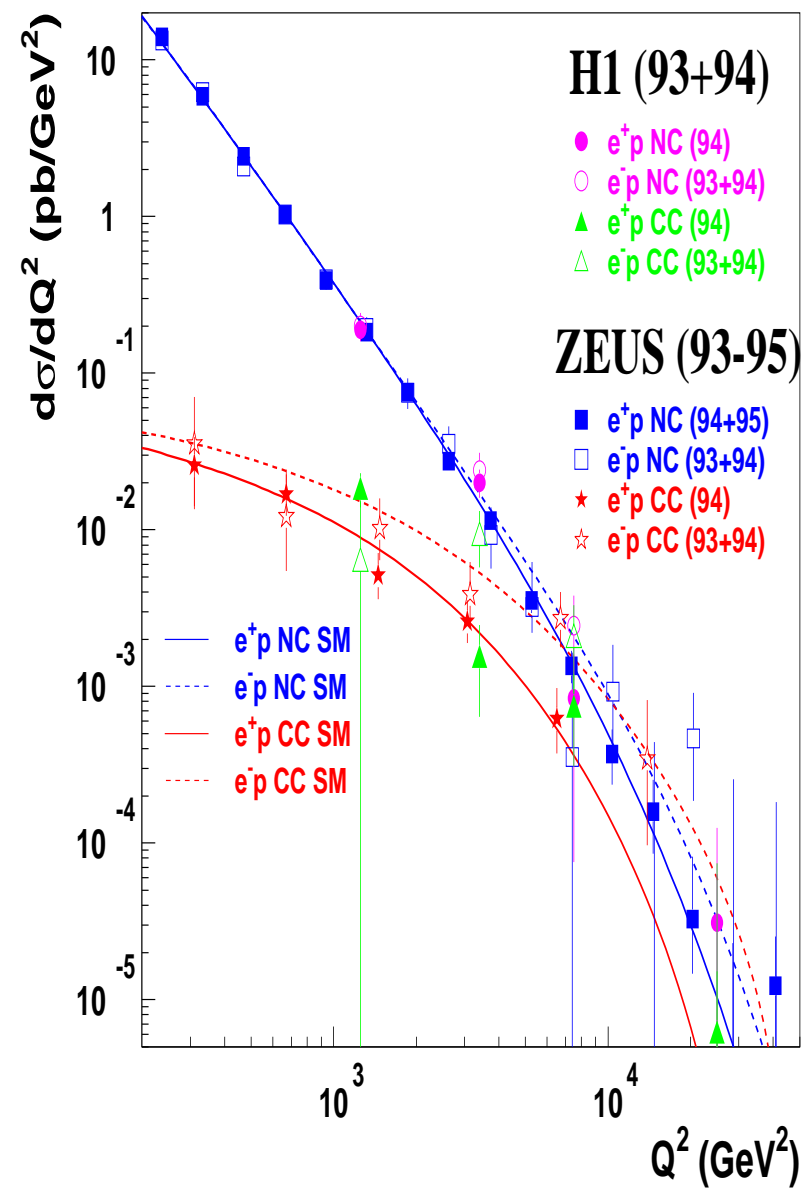

Fig. 77. High $Q^{2} \mathrm{CC}$ and NC cross-sections measured at HERA compared to Standard Model predictions.

effect of varying $\alpha_{s}$ on the resulting PDFs. However this does not give a complete estimate of the uncertainty as both CTEQ and MRS use NLO DGLAP evolution, fit essentially the same data, and use very similar functional forms for the input distributions. As we have noted elsewhere, the problem of the proper inclusion of experimental systematic errors in pQCD fits has been addressed by the HERA experimental groups in their own attempts to extract the gluon momentum distribution 410 . Both $\mathrm{H1}$ and ZEUS have performed NLO QCD fits to extrapolate the fixed target data with $x>0.1$ from SLAC, BCDMS and NMC(97) to the high $Q^{2}$ region. The systematic errors on the data when propagated to the HERA kinematic region $x>0.5, y>0.25$ result in a $6-7 \%$ uncertainty. Varying $\alpha_{s}\left(M_{Z}^{2}\right)$ between 0.113 and 0.123 gives an additional $2 \%$ uncertainty.

\subsection{The high $Q^{2}$ data}


H1 and ZEUS base their analyses on samples of $\mathrm{e}^{+} \mathrm{p}$ DIS collected in the period 1994-96. The full details of the event selections are given in the refs. 407,408 . Both experiments identify the large $Q^{2}$ events by the presence of a beam positron scattered through a large angle, with the primary vertex defined by reconstructed tracks located within a reasonable distance from the nominal interaction point. As discussed in Sec. 4.2 the HERA detectors enable the struck quark jet to be detected as well, thus allowing radiative corrections and photoproduction background to be limited by cuts on the quantity $\sum\left(E-p_{z}\right)$, summed over all calorimeter cells. A brief summary of the cuts and the NC high $Q^{2}$ samples is given in Table 11. In addition to the cuts listed $\mathrm{H} 1$ also requires $0.1<y<0.9$.

Table 11. The NC high $Q^{2}$ data samples from H1 and ZEUS. $E_{\min }^{\prime}$ is the minimum scattered electron energy; $z_{v}$ is the reconstructed primary vertex position along the beam line with its allowed range; the minimum and maximum values of $\sum\left(E-p_{z}\right)$ are given within the brackets. The overall event selection efficiencies for both $\mathrm{H} 1$ and ZEUS are about $80 \%$ for $Q^{2}>Q_{m i n}^{2}$.

\begin{tabular}{cccccccc}
\hline & $\begin{array}{c}\int \mathcal{L} d t \\
{\left[\mathrm{pb}^{-1}\right]}\end{array}$ & $\begin{array}{c}E_{\min }^{\prime} \\
{[\mathrm{GeV}]}\end{array}$ & $\begin{array}{c}z_{v} \\
{[\mathrm{~cm}]}\end{array}$ & $\begin{array}{c}\sum\left(E-p_{z}\right) \\
{[\mathrm{GeV}]}\end{array}$ & $\begin{array}{c}Q_{\min }^{2} \\
{\left[\mathrm{GeV}^{2}\right]}\end{array}$ & $\begin{array}{c}\text { No. } \\
\text { events }\end{array}$ & $\begin{array}{c}\text { SM } \\
\text { expect. }\end{array}$ \\
\hline ZEUS & 20.1 & 20 & \pm 50 & $(40,70)$ & 5000 & 191 & $196 \pm 17$ \\
H1 & 14.2 & 25 & \pm 35 & $(43,63)$ & 2500 & 443 & $427 \pm 38$ \\
\hline
\end{tabular}

Note that for these samples there is no discrepancy with the Standard Model expectations. Also the difference in event numbers for the two experiments is explained by the differences in integrated luminosities and cut values, particularly $Q_{\min }^{2}$. For reconstruction of event kinematics, H1 use the electron method and ZEUS the double-angle method but each experiment uses the other as a systematic check. For both methods at large $Q^{2}$ the most important detector uncertainty comes from the absolute energy calibration of the calorimeters and for both experiments it amounts to about $3 \%$. Other systematic uncertainties in the measurement of the event sample are $2.3 \%$ in the luminosity determination and about $2 \%$ in the radiative correction estimate. Taking these uncertainties together with the cross-section uncertainty above gives a total systematic error of about $8.5 \%$ for each experiment. Because the energy scale uncertainty has a bigger effect at large $Q^{2}$, the systematic error may reach $30 \%$ for the largest $Q_{e}^{2}$, which is why it is so important that both reconstruction methods be used. When the samples are studied in more detail it is found that there is an excess of events above the Standard Model expectation in the region of large $x$ and $y$.

The ZEUS group analyse their data using kinematic variables $Q^{2}, x, y$ reconstructed using the double angle method. Fig. 78 shows the $Q^{2}$ distribution for the ZEUS data with $y>0.25$ compared to the SM expectation. An excess of events is visible for $Q^{2}>35000 \mathrm{GeV}^{2}$. H1 use the variables $Q^{2}, y$ and $M=\sqrt{x s}$ ( $M$ is the invariant mass of the $e-q$ system) reconstructed using the electron method. Fig. 79 shows the distribution in $M$ of the H1 sample compared to the SM expectation for $y>0.2$ and $y>0.4$. There is an excess of events in the bin centered on $200 \mathrm{GeV}$. 


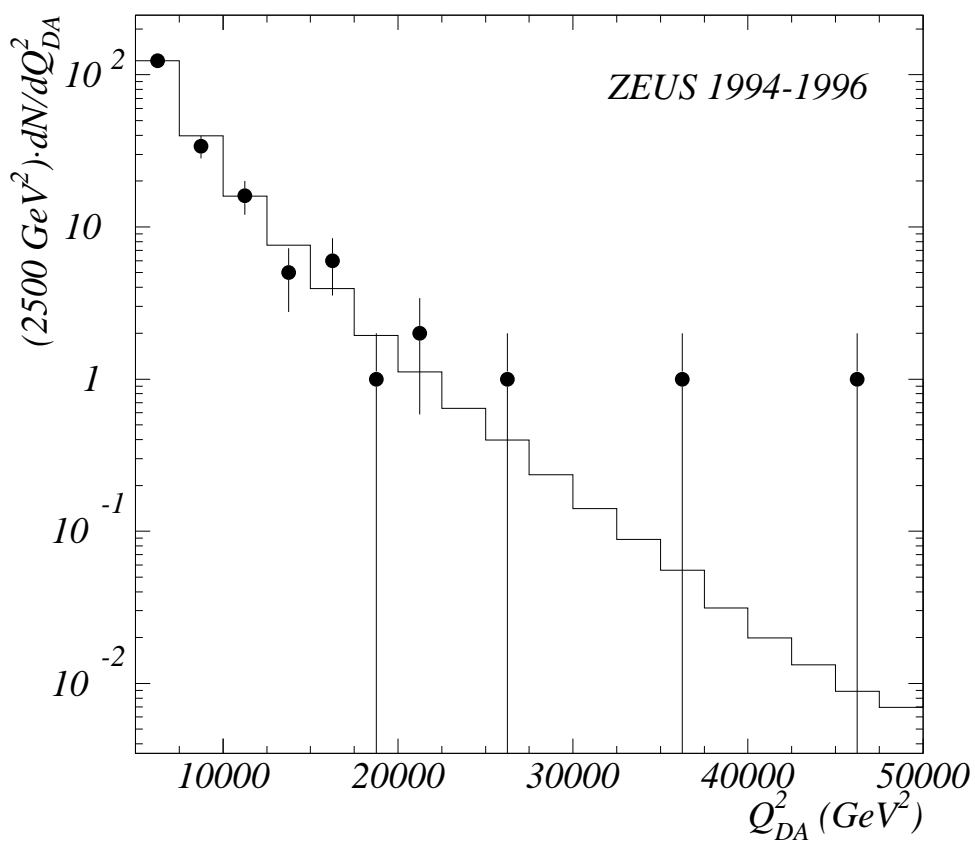

Fig. 78. The $Q^{2}$ distribution for the ZEUS high $Q^{2}$ sample with $y>0.25$ compared to the SM expectation.

In more detail, ZEUS observe 2 events with $Q^{2}>35000 \mathrm{GeV}^{2}$ where 0.15 are expected, giving a Poisson probability of $0.39 \%$ (for 2 or more) and for $x>0.55$ and $y>0.25$, ZEUS observe 4 events where 0.91 are expected (probability of $0.60 \%$ for 4 or more). Using simulated experiments ZEUS find that, for a luminosity of $20.1 \mathrm{pb}^{-1}$, there is a $6.0 \%$ probability to observe a fluctuation of $0.39 \%$ or less in $Q^{2}$ for any $Q^{2}$ in the range. For $Q^{2}>15000 \mathrm{GeV}^{2} \mathrm{H} 1$ observe 12 events where 4.7 are expected with a probability of $0.6 \%$ (for 12 or more) and for the interval $187.5<$ $M<212.5 \mathrm{GeV}$ with $y>0.4,7$ events are observed where 0.95 are expected with a probability of $0.4 \%$ (for 7 or more). Using simulated experiments for a luminosity of $14.2 \mathrm{pb}^{-1} \mathrm{H} 1$ find that there is a $1 \%$ probability to observe a fluctuation to the minimum or less (averaged over windows of $25 \mathrm{MeV}$ ) for any mass in the $M$ range 80 to $250 \mathrm{GeV}$ Full details for each of the $7 \mathrm{H} 1$ and 4 ZEUS 'excess' events are given in Refs. 407,408

$\mathrm{H} 1$ have in addition measured the $\mathrm{e}^{+} \mathrm{p} \mathrm{CC}$ cross-section at high $Q^{2}$. The crucial event selection cut is to require a total missing transverse momentum of at least $50 \mathrm{GeV}$, giving a sample of $31 \mathrm{CC}$ events with $Q^{2}>2500 \mathrm{GeV}^{2}$ compared to a SM expectation of 34 events. For $Q^{2}>20000 \mathrm{GeV}^{2} \mathrm{H} 1$ find 3 events where 0.74 are expected, with a probability of $5 \%$. 

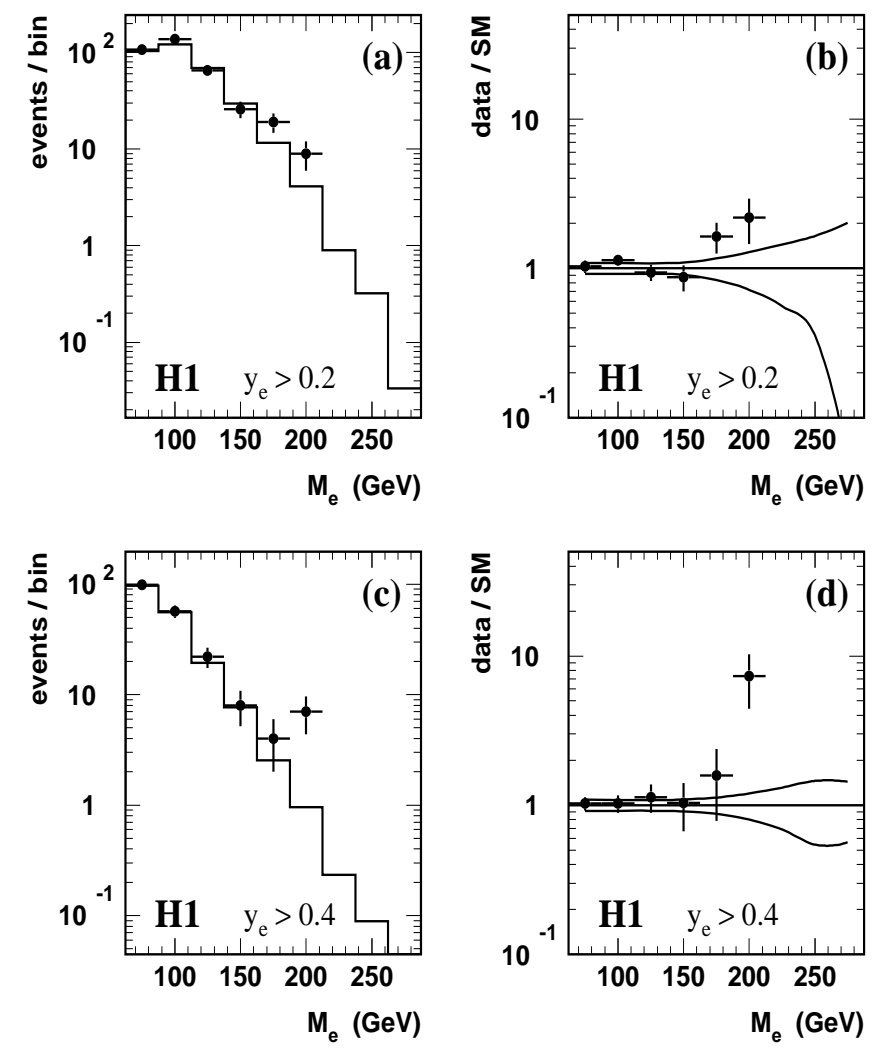

Fig. 79. The distribution in $M=\sqrt{x s}$ of the $\mathrm{H} 1$ high $Q^{2}$ sample for $y>0.2$ and $y>0.4$.

\subsection{Possible explanations}

First could the excess be due to background? Apart from misidentified QED Compton scattering and weak boson production, the most dangerous background processes are all related to photoproduction in which an electromagnetic cluster of large energy (probably from $\pi^{0}$ decay) fakes the scattered electron. Visually the events in the region of the excess are very clean with an isolated electron balanced by a single hadronic jet. On a more quantitative level ZEUS estimate less than 1 event out of 46 observed in the large $Q^{2}$ sample (restricted to $x>0.55$ ) from all background channels and $\mathrm{H} 1$ estimate less than 0.1 event $(95 \% \mathrm{CL})$ from all background sources in their sample of 20 events with $Q^{2}>10000 \mathrm{GeV}^{2}$.

The effect could be a statistical fluctuation. This explanation awaits the increased statistics expected from the long HERA run in 1997.

One of the first questions to be addressed is are the effects from the two experiments compatible? The answer at present is yes but only just. The 7 excess events 
from H1 cluster around a mass value of about $200 \mathrm{GeV}(M=\sqrt{x s})$ whereas the 4 ZEUS events have mass values spread between 230 and $250 \mathrm{GeV}$. The difference in mean mass is larger than the difference expected from using different methods of reconstruction and both groups have checked that their results are internally consistent by using the other reconstruction method, electron in place of doubleangle for ZEUS and vice-versa for H1. Another possible cause of spread is initial state radiation from the beam positron, though it is unlikely that this would affect more than a few of the events. ISR affects the electron and double-angle methods differently, and in fact if both methods are used on the same events the energy of the radiated photon can be reconstructed, assuming that is collinear with the positron beam. The details have been discussed in general terms in a recent paper by Wolf 411 and specifically for these events by Bassler \& Bernardi 112 . The latter authors also propose a modification of the $\Sigma$ method (see Sec. 4.2.1) which improves the resolution somewhat at very large $Q^{2}$. They use the method on the combined event sample, correcting two events for ISR and find that although the difference in mean mass between the ZEUS and $\mathrm{H} 1$ events is reduced from $26 \pm 10$ to $17 \pm 7 \mathrm{GeV}$, it is still larger than one would expect from the decay of a single narrow resonance.

A number of authors have considered various ways in the which the parton distributions could be modified at large $x$ without altering the good description of low $Q^{2}$ fixed target data. Note that, because of the feed-down effect in $x$ as one evolves up in $Q^{2}$, any changes have to be made well above the $x$ region at which the excess has been observed at HERA. Kuhlmann, Lai \& Tung 113 , within the context of the CTEQ global fit, investigate how large a change would be required to the valence quark distributions to fit the HERA excess. They first establish that the effect of changing the parameters of the standard $u_{v}$ distribution to increase its magnitude for $x>0.75$ is not nearly sufficient. They then show that adding an extra component to $u_{v}$ of the form $0.02(1-x)^{0.1}$ at $Q_{0}^{2}$ produces an increase at $x \approx 0.5-0.6$ of about the right size to explain the number of excess events. Of course by its nature such a modification predicts an even larger increase in the cross-section at larger $x$ values. They do not attempt to look for any justification for the extra term and point out that if the effect is associated with a narrow structure or structures then this sort of modification would not be appropriate.

Rock and Bosted 414 suggest that one might learn something about $F_{2}$ in the range $0.7 \leq x \leq 0.97$ from SLAC $e p$ data taken in the $N^{*}$ resonance region. Their idea is to use Bloom-Gilman duality 415 which states that the average of the structure function in the resonance region is approximately equal to its value in DIS. They compare SLAC data to the NMC parametrization (Eq. 121) and to the recent CTEQ4M global fit and find that for $x>0.7$ the average resonance data give a value of $F_{2}$ about one order of magnitude above the conventional fit. This is however still roughly an order of magnitude below what is required to explain the excess at high $Q^{2}$. The authors warn of the care necessary at large $x$ as $F_{2}$ is decreasing rapidly, but argue that the form of the fit parametrization should perhaps be looked at again including allowance for higher twist terms. 
Gunion \& Vogt 416 point out that an intrinsic charm component in the proton could give rise to an excess of events localised in the $e-q$ mass near $200 \mathrm{GeV}$. The intrinsic charm model is formulated in terms of nucleon Fock states 269 and in particular the state $|u u d c \bar{c}\rangle$, will be important at large $x$ values. Existing constraints limit the size of this contribution to only $15 \%$ in the $e^{+} p$ NC channel, but could be much larger in the CC channel. So, if the size of the HERA excess in the $\mathrm{NC}$ channel is confirmed, then their version of intrinsic charm would not be a viable explanation. An alternative version of the intrinsic charm approach has been suggested by Melnitchouk and Thomas 417 in the context of the meson cloud model for long-range nucleon structure. In this the charm sea component is taken to arise from quantum fluctuations to identified states - for example $D^{-}+\Lambda_{c}$. Using this approach the authors show that they can produce a larger effect than that of Gunion \& Vogt. However Melnitchouk \& Thomas are not claiming that this is the explanation of the HERA excess but merely that there is a SM approach with some physical basis which could provide a cross-section of about the right magnitude without violating the standard PDF description of data at lower $Q^{2}$. The direct investigation of charm in DIS is very much on the agenda for study at HERA so as luminosity increases it may be possible to answer some of the questions raised by these authors directly.

Szczurek and Budzabowski 118 have studied whether the effect of a meson cloud and the inclusion of target mass corrections in the description of the low $Q^{2}$ DIS data may, after QCD evolution, influence the large $x$ and large $Q^{2}$ region. Meson cloud effects, i.e. the admixture of $\pi N, \pi \Delta$ and similar components in the Fock expansion of the proton wave function, have been put forward as a way of understanding the Gottfried Sum Rule, the measured Drell Yan asymmetries in $p p$ and $p d$ collisions and semi-inclusive production of fast neutrons at HERA. The inclusion of such effects at low $Q^{2}$ leads to an enhancement of the quark distributions at large $x$, which survives QCD evolution to the HERA high $Q^{2}$ region. For $Q^{2}>10^{4} \mathrm{GeV}^{2}$ the predicted increase due to these effects is $30 \%(100 \%)$ for $x=0.7(0.9)$. When integrating the region $x>0.5(0.7)$ the relative enhancement of the cross section due to meson cloud effects and target mass corrections for $Q^{2}=10^{4} \mathrm{GeV}^{2}$ is $20 \%$ $(60 \%)$, slowly increasing with $Q^{2}$. Hence it is unlikely that these mechanisms can provide a complete explanation of the high $Q^{2}$ effect, but they need to be considered when analysing future high $Q^{2}$ data at large $x$.

Kochelev 419 suggests the possibility that the excess of events at high $Q^{2}$ is due to an instanton contribution to the proton structure function. Instantons are nonperturbative strong vacuum fluctuations, which describe the tunnelling between different gauge rotated vacua in QCD. A characteristic feature is that they yield a large multi-gluon final state in DIS. The instanton contribution does not evolve with $Q^{2}$ at the same rate as the standard PDF contribution and this leads to a relative increase in the instanton fraction at large $x$ and $Q^{2}$. First estimates suggest, albeit with large uncertainties, that the instanton contribution in the region of the high $Q^{2}$ HERA events can be $50 \%$ or more, and would therefore increase the SM prediction 
considerably. Due to the specific nature of the instanton final state in DIS, which includes flavour democracy, the study of such final state properties as energy flow and strange or charm particle abundances could provide decisive information on this scenario.

The most exciting possibility is that one is seeing the hint of something beyond the Standard Model. The HERA observations have unleashed a flood of theoretical speculation which it would not be appropriate to summarise here. A compact survey of the field has been given by Dokshitzer 275 in his summary of theoretical developments at the DIS97 Workshop. The models are in three broad categories: contact interactions, leptoquarks or an $R$-parity violating supersymmetric squark; all are conveniently summarised in the paper by Altarelli et al 120 , in which existing constraints are also discussed. Contact interactions are a way of describing the effect of new physics at a scale well below that of the new force quanta (as the Fermi theory approximates the full electroweak theory for low $Q^{2} \mathrm{CC}$ interactions). The effective Lagrangian for the four-point interaction is of the form

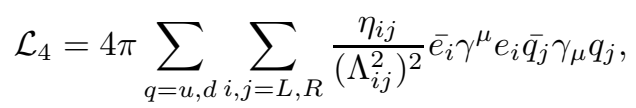

where $\Lambda_{i j}$ is the mass scale and coupling parameter and $\eta_{i j}= \pm 1$ allows for destructive and constructive interference. Contact interactions will generate an excess of events as $Q^{2}$ increases, but not a structure in $x$. There are of course limits on contact interactions from $e^{+} e^{-}$interactions at LEP and p $\bar{p}$ interactions at the Tevatron, but there are still a few possibilities which could just about explain the HERA excess with mass scales in the range $1.7-2.5 \mathrm{TeV}$ depending on the sign and helicity of the coupling. If the effect is concentrated around a specific $M=\sqrt{x s}$ value then the most natural explanation is either a leptoquark (an electron-quark bound state) or an $R$-parity violating squark coupling to $e-q$. There are many possible leptoquarks and they are classified by spin and isospin quantum numbers. An $e^{+} u$ or $e^{+} d$ state with standard Yukawa couplings and a mass of about $200 \mathrm{GeV}$ is not yet ruled out by Tevatron data but such an exclusion is getting close. One would not expect such a scalar leptoquark to decay to the $\nu \bar{q}$ final state, whereas a vector one could. Perhaps the most favoured explanation is that of an $R$-parity violating squark coupling to valence $d$ quarks ( $e^{+} d$, such as stop) or one coupling to the $\bar{u}$ sea $\left(e^{+} \bar{u}\right)$. When one takes into account limits from other high energy processes and atomic parity violation experiments, the most favoured options are left-handed scalar charm or top squarks.

\subsection{August 1997 update}

For the Hamburg Lepton-Photon Symposium 421 and the Jerusalem EPS HEP conference ${ }^{222}$, the HERA high $Q^{2} \mathrm{NC}$ results were updated with the addition of data collected to the end of June 1997. This has increased the accumulated luminosity by roughly $67 \%$ (to $23.7 p b^{-1}$ for $\mathrm{H} 1$ and $33.5 p b^{-1}$ for ZEUS). Unfortunately the new data have not clarified the experimental picture very much. The measured NC cross- 
sections are still in excess of the SM expectation, but the number of 'signal' events has only increased slightly. For H1, in the mass range $187.5<M<212.5 \mathrm{GeV}$, 8 events are now observed where $1.53 \pm 0.29$ are expected with probability $0.3 \%$ (compared to 7 observed and 0.95 expected in the earlier report). For ZEUS, with $x>0.55$ and $y>0.25,5$ events are observed where $1.51 \pm 0.13$ are expected with a probability of $1.9 \%$ (compared to 4 observed and 0.91 expected before). Preliminary $\mathrm{CC}$ cross-sections for the same total luminosities were also presented, the results from both experiments are above the SM expectation, for $Q^{2}>10^{4} \mathrm{GeV}^{2} \mathrm{H} 1$ and ZEUS together observe 28 events where $17.7 \pm 4.3$ are expected. The tentative conclusion that a single narrow resonance structure in $x$ cannot explain the data from both experiments has been reinforced. From the discussion in the previous section on uncertainties in and novel models for the PDFs at large $x$, it is also clear that more work needs to be done on the calculation of the standard model NC and CC cross-sections at large $x$ and $Q^{2}$. Full analyses of all high $Q^{2}$ data collected up to the end of 1997 running (estimated to be totals of 38 and $48 \mathrm{pb}^{-1}$ for $\mathrm{H1}$ and ZEUS respectively), which more than double the statistics of the original publications, are expected in early 1998.

\section{Summary and outlook}

In this review we have concentrated on the complete data sets from the latest round of muon beam fixed target experiments, NMC at CERN and E665 at FNAL, and the first substantial data on proton structure from the two HERA general purpose experiments, H1 and ZEUS. We have covered briefly the revised neutrino beam data (on an iron target) from CCFR at FNAL. The CCFR data are important because they are the most accurate measurements of $\nu N$ structure functions and they provide a direct measurement of the non-singlet component from which $\alpha_{s}$ has been determined free of the convolution with the gluon momentum distribution. The NMC and E665 data on $F_{2}^{p}$ and $F_{2}^{d}$ are consistent with each other and with the earlier high statistics measurements on $e N$ scattering at SLAC and the BCDMS $\mu N$ experiment at CERN. There is still a discrepancy at low $x$ between the $F_{2}^{p}$ values derived from CCFR data and those of NMC and E665. In the design of both E665 and NMC special attention was paid to efficient triggering and kinematic reconstruction at very small angles in the laboratory allowing the measurement of structure functions at small $x$. The push to small $x$ has seen a natural continuation with the data from the HERA collider, in which the $e p$ centre of mass energy has increased by an order of magnitude (from $20-30 \mathrm{GeV}$ to $300 \mathrm{GeV}$ ). The $F_{2}^{p}$ data from $\mathrm{H} 1$ and ZEUS are consistent with each and match smoothly to the fixed target data, though the region of direct overlap is small. At HERA the measurement of $F_{2}$, or equivalently $\sigma^{\gamma^{*} p}$, at very small values of $Q^{2}$ has been performed either by shifting the $e p$ interaction point and/or by the use of dedicated small electromagnetic calorimeters near the beam line. The data provides almost complete coverage from $Q^{2}=0$ to the 'safely' deep inelastic region with $Q^{2}>4 \mathrm{GeV}^{2}$ and has enabled precision studies of the transition region in which the description by pQCD breaks 
down.

New measurements of $R$ have been made by both NMC and CCFR. If $R$ has not been measured in the experiment then the teams have taken a consistent approach on how to handle the correction for $R$ in the extraction of $F_{2}$ from the cross-sections. For the fixed target experiments the SLAC parametrization of $R$ has been used and for HERA experiments $R_{Q C D}$ has been used. The systematic errors of the fixed target experiments are in the range $2-3 \%$ and those from HERA around $5 \%$. We now have data on $F_{2}$ in the ranges $3 \cdot 10^{-6}<x<0.9$ and $0.11<Q^{2}<5000 \mathrm{GeV}^{2}$.

The expansion of the kinematic region to very low $x$ at $Q^{2}$ values of at least a few $\mathrm{GeV}^{2}$ has transformed the study of gluon dynamics within QCD. Two major facts about $F_{2}$ have emerged from the HERA measurements: the first is that $F_{2}$ rises as $x$ decreases, the rise increasing as $Q^{2}$ increases and the second is that standard NLO QCD appears to describe the data from the highest $Q^{2}$ values to the surprisingly low value of $1-2 \mathrm{GeV}^{2}$. It is agreed that the rise of $F_{2}$ at low $x$ is caused by an increase in the $q \bar{q}$ sea which in turn is being driven by a rapid increase in the gluon density. Much theoretical effort has been expended on trying to understand exactly what the dynamical mechanism is. So far there is no definitive answer. Early on some argued strongly that the data would require the use of BFKL inspired multi-gluon dynamics giving singular parton distributions at a conventional starting scale of $Q_{0}^{2} \approx 4 \mathrm{GeV}^{2}$, while others pointed to the success of the GRV approach using a very low starting scale, $\sim 0.3 \mathrm{GeV}^{2}$, with valence-like non-singular input distributions. The success of Double Asymptotic Scaling as advocated by Ball and Forte also indicated that conventional DGLAP evolution could describe the data. However, as the precision and extent of the data improved it became apparent that he GRV94 parametrization itself cannot describe all the low $x$ data and that Double Asymptotic Scaling needs corrections within a full NLO calculation. The legacy of the GRV approach is that most successful fits to data within the conventional framework use a low starting scale, $Q^{2} \sim 1 \mathrm{GeV}^{2}$, with non-singular inputs.

However, much work continues on the question whether or not BFKL dynamics are necessary to describe the HERA data. The phenomenologically successful nonconventional approaches incorporating (and going beyond) BFKL dynamics are: the resummation of $\ln (1 / x)$ terms using 'physical' anomalous dimensions as advocated by Catani and implemented by Thorne; unified DGLAP-BFKL approaches based on the unintegrated gluon distribution, such as the CCFM equation and modified BFKL equations; and attempts to solve the BFKL equation within the colour dipole approach. Thus we have a situation whereby both conventional and nonconventional QCD dynamics can fit the data for $Q^{2}>1 \mathrm{GeV}^{2}$, and the difference in $\chi^{2}$ of the fits to $F_{2}$ data is not sufficiently large to allow us to make a clear decision.

We have clearly reached a region where $\alpha_{s} \ln (1 / x) \sim 1$ so that conventional expansions in $\alpha_{s}$ should no longer be reliable. The fact that conventional approaches are successful thus needs some explanation. We have summarized some of the possible explanations in Sec. 7.6. Here we recall that recent theoretical work suggests that a full treatment of $N L L(1 / x)$ effects within the BFKL equation would lead 
to a softening of the BFKL Pomeron such that it becomes more consistent with the behaviour predicted by the conventional expansions. Camici and Ciafaloni have even suggested that a full understanding of $N L L(1 / x)$ contributions would lead to an understanding of the transition to soft physics as $Q^{2} \rightarrow 0$. It is also possible to imagine a 'conspiracy' whereby higher twist terms and shadowing corrections at low $x$ could be large, even at moderate $Q^{2}$, hiding BFKL effects. Finally there is always the nagging doubt that the freedom to choose a fairly arbitrary function of $x$ for the input parton distributions may be hiding a real breakdown of the standard description. Hence it is very important that the efforts to derive information about the input parton distributions using non-perturbative techniques, such as the lattice and chiral dynamics, continue to be pursued vigorously.

Some of these questions should find answers when different variables affected by non-conventional dynamics are measured. The measurements from the hadron final state already give tantalizing glimpses of new dynamics. We look forward to accurate measurements of structure functions other than $F_{2}$. Measurements of $F_{L}$ will be made in the HERA region, when increased luminosity allows us to exploit the ISR method, or, by use of different beam energies. The period covered by this review has seen the publication of the first measurements of the charm structure function, $F_{2}^{c \bar{c}}$, at HERA from a DIS event sample containing identified $D$ and $D^{*}$ mesons, and we look forward to more accurate measurements in future. There has also been a major advance in the theoretical treatment of heavy flavours in DIS with the calculation to NLO of the massive quark coefficient functions. This has in turn stimulated a lively debate on the correct way to handle heavy flavours, in particular charm, through the threshold region, where the mass effects are obviously important, to large $Q^{2}$, where the mass is no more important than that of the light quarks. The jury is still out.

In the immediate future we expect to see more data on $F_{2}$ from the HERA experiments. The data that we have reported on was collected in 1994 when HERA delivered $6 \mathrm{pb}^{-1}$. When quality checks and detailed cuts are made typically about $50 \%$ of the delivered luminosity is used for cross-section measurement and structure function determination. In 1995, 1996 and 1997 HERA delivered 12, 17 and $36 \mathrm{pb}^{-1}$, respectively. If the full samples from 1995-97 are used then the $Q^{2}$ at which systematic errors dominate will increase from around $100 \mathrm{GeV}^{2}$ now to above $500 \mathrm{GeV}^{2}$. The aggregated sample should allow of initial studies of $F_{L}$ using the ISR method. The understanding of systematic errors will also improve as detector studies can be made with finer geometrical binning. The number of identified DIS charm events will also increase from higher luminosity and from more efficient methods of tagging, particularly from the use of micro-vertex detectors. H1 has one installed and has taken some data with it, ZEUS hopes to install one for operation in the year 2000. The urgent question of whether the excess of HERA DIS events seen at very high $Q^{2}$ is simply a statistical fluctuation or not should be resolved. If the anomaly remains it will require much more high luminosity at high $Q^{2}$ for its complete understanding. Also in the future is the prospect of running with 
polarized $\mathrm{e}^{ \pm}$beams and possibly polarized protons. The option of running with deuterons instead of protons is also under consideration. It is planned to upgrade the luminosity of the HERA collider incrementally between now and the year 2000, many details of this and the physics that will then become possible can be found in the report of the 1996 Workshop on Future Physics at HERA 423.

Apart from HERA we have the prospect of the NuTeV experiment at Fermilab. Thus the next five years should see a further significant increase in our understanding of the nature of parton distributions and the forces which shape them. Deep inelastic scattering and hadronic structure functions are still vibrant and important fields of study and uniquely important tools in unravelling the complexities of QCD 30 years after such methods first led us to the dynamical quark-parton model.

\section{Acknowledgements}

Many people have helped us to accumulate information for this review. For advice on the data and helping to prepare plots we thank: H. Abramowicz, A. Bodek, G. Contreras, E. Kabuss, M. Kuhlen, F. Lehner, A. Levy, A. Quadt, E. Rondio, W. Seligman, W. Smith, B. Surrow, J. Tickner, U. Yang. For many discussion on the complexities of QCD and small $x$ physics we thank R. Ball, A. D. Martin, R. G. Roberts, R. Thorne and for supplying original figures we thank B. R. Webber, Ch. Royon, P. Sutton. We thank M. Lancaster for reading and commenting on an early version of the manuscript.

\section{References}

1. Symposium on Lepton and Photon Interactions, Hamburg July 1997, http://www.desy.de/lp97/.

2. EPS HEP97 Conference, Jerusalem, August 1997, http: //www . cern. ch/hep97/.

3. E. Gallo, invited talk at Proc. of the 1997 Symposium on Lepton Photon Interactions, Hamburg, July 1997, eds. A. De Roeck and A. Wagner, hep-ex/9710013.

4. R. Eichler, invited talk at EPS/HEP Conference 97, Jerusalem, August 1997, http://www . cern.ch/hep97/.

5. F. Halzen and A.D. Martin, Quarks and Leptons, John Wiley, 1984.

6. R.G. Roberts, The Structure of the Proton, CUP 1990.

7. C.G. Callan and D. Gross, Phys. Rev. Lett. 22, 156 (1969).

8. R.P. Feynman, Phys. Rev. Lett. 23, 1415 (1969);

Photon Hadron Interactions, Benjamin N.Y. 1972.

9. J.D. Bjorken, Phys. Rev. 179, 1547 (1969).

10. J.I. Friedman, H.W. Kendall \& R.E. Taylor, Rev. Mod. Phys. 63, 573,597,615 (1991).

11. H.E. Fisk \& F. Sciulli, Ann. Rev. Nucl. Part. Sci. 32, 499 (1982).

12. D.J. Gross and C.H. Llewellyn-Smith, Nucl. Phys. B 14, 337 (1969).

13. S.L. Adler, Phys. Rev. 143, 1144 (1966).

14. K. Gottfried, Phys. Rev. Lett. 18, 1154 (1967).

15. H. Abramowicz et al, Z. Phys. C 17, 283 (1983).

16. S.J. Brodsky and G. Farrar, Phys. Rev. Lett. 31, 1153 (1973).

17. A. Donnachie and P.V. Landshoff, Phys. Lett. B 296, 257 (1992).

18. J.R. Forshaw and D.A. Ross, QCD and the Pomeron, CUP 1997. 
19. see for example, A.Buras, Rev.Mod.Phys 52, 199 (1982).

20. M.R. Pennington, Phys. Rev. D 26, 2048 (1982);

P.M. Stevenson, Phys. Rev. D 23, 2916 (1981).

21. Yu. Dokshitzer, Soviet Phys. JETP 46, 641 (1977);

V.N. Gribov and L.N. Lipatov, Soviet J. Nucl. Phys. 15, 438,675 (1972);

L.N. Lipatov, Soviet J. Nucl. Phys. 20, 95 (1975);

G. Altarelli and G. Parisi, Nucl. Phys. B 126, 298 (1977).

22. P. Renton, Electroweak Interactions, CUP, 1990.

23. A.M. Cooper-Sarkar et al, Z. Phys. C 39, 281 (1988);

A.M. Cooper-Sarkar et al, Proc. of the Workshop on Physics at HERA, DESY 1991, eds W. Buchmüller \& G. Ingelman, Vol I, p155.

24. F.J. Yndurain, Quantum Chromodynamics, Springer-Verlag 1983.

25. H. Georgi and H.D. Politzer, Phys. Rev. D 14, 1829 (1976);

O. Nachtmann, Nucl. Phys. B 63, 237 (1973).

26. High $p_{t}$ physics and higher twists, Nucl.Phys.Proc.Supp 7B, (1989).

27. J.L. Miramontes, M.A. Miramontes and J. Sanchez-Guillen,

Phys. Rev. D 40, 2184 (1989);

R.K. Ellis, W. Furmanski and R. Petronzio, Nucl. Phys. B 212, 29 (1980);

E.V. Shuryak and A.I. Vainshtein, Nucl. Phys. B 199, 451 (1982).

28. V.M. Braun and A.V. Kolesnichenko, Nucl. Phys. B 283, 723 (1987);

G.G. Ross and R.G. Roberts, Phys. Lett. B 322, 425 (1994);

I.I. Balitsky, V.M. Braun and A.V. Kolesnichenko, Phys. Lett. B 242, 245 (1990), and Phys. Lett. B 318, 648 (1993).

29. A. Harindranath et al, hep-ph/9711298.

30. B.R. Webber, Proc. of DIS96, Rome, Eds G. D'Agostini \& A. Nigro, World Scientific 1997, p77.

31. M. Dasgupta and B.R. Webber, Phys. Lett. B 382, 273 (1996);

M. Maul et al, hep-ph/9710531.

32. G. Sterman, Summary talk at DIS97, Chicago http: //www.hep.anl.gov/dis97/.

33. W.J. Marciano, PRD 29, 5801 (1984).

34. E. Laenen et al, Proc. of the Workshop on Future Physics at HERA, DESY 1996, eds G. Ingelman, A. De Roeck \& R. Klanner, Vol. I, p393;

Wu-Ki Tung, Proc. of DIS97, Chicago, http://www.hep.anl.gov/dis97/; M.Buza et al, hep-ph/9707263;

W.L.van-Neerven, hep-ph/9708452;

J.C.Collins, M.G. Ryskin and A.D. Martin, hep-ph/9709440.

35. A.D. Martin, R.G. Roberts and W.J.Stirling, Phys. Rev. D 47, 867 (1993).

36. A.D. Martin, R.G. Roberts and W.J.Stirling, Phys. Rev. D 50, 6734 (1994).

37. A.D. Martin, R.G. Roberts and W.J.Stirling, Phys. Lett. B 387, 419 (1996).

38. M. Gluck, E. Reya and A Vogt, Z. Phys. C 48, 471 (1990), Z. Phys. C 53, 127 (1992).

39. M. Gluck, E. Reya and A. Vogt, Z. Phys. C 67, 433 (1995).

40. M. Gluck, E. Reya and M. Stratmann, Nucl. Phys. B 422, 37 (1994).

41. J.P Leveille \& T. Weiler, Nucl. Phys. B 147, 147 (1979)

42. E. Laenen et al, Nucl. Phys. B 291, 325 (1992); ibid 392, 162,229 (1993);

S. Riemersma, J. Smith \& W.L. van Neerven, Phys. Lett. B 347, 143 (1995).

43. A. Vogt, Proc. of DIS96, Rome, Eds G. D'Agostini \& A. Nigro, World Scientific 1997, p254.

44. H. L. Lai and W.K. Tung, Z. Phys. C 74, 463 (1997).

45. A.D. Martin et al, hep-ph/9612449.

46. M.A.G. Aivazis et al, Phys. Rev. D 50, 3102 (1994). 
47. C.R. Schmidt, Proc. of DIS97, Chicago, hep-ph/9706496.

48. M. Buza et al, hep-ph/9612398.

49. F.I. Olness and R.J. Scalise, Proc. of DIS97, Chicago, hep-ph/9707459.

50. R.G. Roberts and R.S. Thorne, hep-ph/9709442, hep-ph/9711223.

51. S.R. Mishra \& F. Sciulli, Ann. Rev. Nucl. Part. Sci. 39, 259 (1989).

52. T. Sloan, G. Smadja \& R. Voss, Phys. Rep. 162, 45 (1988).

53. NMC, P. Amaudruz et al, Nucl. Phys. B 371, 3 (1992);

I.G. Bird, PhD Thesis, Free University of Amsterdam (1992).

54. R.P. Mount, Nucl. Instrum. Methods 187, 401 (1981).

55. E665, M.R. Adams et al, Nucl. Instrum. Methods A 291, 533 (1990).

56. W.K. Sakumoto et al, Nucl. Instrum. Methods A 294, 179 (1990);

B.J. King et al, Nucl. Instrum. Methods A 302, 254 (1991).

57. CCFR, R. Blair et al, Phys. Rev. Lett. 51, 343 (1983);

P. S. Auchinloss et al, Z. Phys. C 48, 411 (1990);

CDHSW, P. Berge et al, Z. Phys. C 49, 187 (1991).

58. S. Bentvelsen, J. Engelen \& P. Kooijman, Proc. of the Workshop on Physics at HERA, DESY 1991, eds W. Buchmüller \& G. Ingelman, Vol I, 23;

S. Bentvelsen, PhD Thesis, University of Amsterdam 1994.

59. F. Jacquet \& A. Blondel, Proc. of the Study of an ep facility for Europe, ed U Amaldi, DESY 79/48, p391.

60. H1, I. Abt et al, NIMA 386, 310 (1997).

61. H1, Technical proposal for the upgrade of the rear region of the H1 detector, DESY Internal Report PRC-93/02.

62. H1, S. Aid et al, Nucl. Phys. B 470, 3 (1996).

63. H1, C. Adloff et al, DESY preprint DESY 97-042.

64. U. Bassler \& G. Bernardi, Nucl. Instrum. Methods A 361, 197 (1995).

65. ZEUS, M. Derrick et al, Phys. Lett. B 293, 465 (1992);

The ZEUS Detector Status Report 1993, DESY 1993;

M. Derrick et al, Z. Phys. C 63, 391 (1994).

66. W.H. Smith et al, Nucl. Instrum. Methods A 355, 278 (1995).

67. ZEUS, M. Derrick et al, Z. Phys. C 72, 399 (1996).

68. L.W. Whitlow et al, Phys. Lett. B 250, 193 (1990).

69. L.W. Mo \& Y.S. Tsai, Rev. Mod. Phys. 41, 205 (1969);

Y.S. Tsai, SLAC preprint SLAC-PUB-848, 1971.

70. A.A. Akhundov et al, Soviet J. Nucl. Phys. 26, 660 (1977);

A.A. Akhundov et al, Proc. 1992 Zeuthen Workshop on Elementary Particle Theory, eds J. Blümlein \& T. Riemann, p209 and references therein.

71. Report of the Working Group on Radiative Corrections, Proc. of the Workshop on Physics at HERA, DESY 1991, eds W. Buchmüller \& G. Ingelman, vol II, 787.

72. A. Arbuzov et al, Comp. Phys. Comm. 94, 128 (1996).

73. J. Blümlein, Proc. of the Workshop on Physics at HERA, DESY 1991, eds W. Buchmüller \& G. Ingelman, vol III, 1272.

74. A.A. Akhundov et al, ibid, 1285.

75. K. Kwiatkowski, H. Spiesberger \& H-J. Möhring, ibid, 1294.

76. G.A Schuler \& H. Spiesberger, ibid, 1419.

77. G. Ingelman, Proc. of the Workshop on Physics at HERA, DESY 1991, eds W. Buchmüller \& G. Ingelman, vol III, 1366.

78. L. Lönnblad, Comp. Phys. Comm. 71, 15 (1992); Z. Phys. C 65, 285 (1995).

79. B. Badelek et al, J. Phys. G. 19, 1671 (1993).

80. A. V. Kotwal, Harvard Thesis 1995.

81. D. Bardin et al, Proc. of the Workshop on Future Physics at HERA, DESY 1996, 
eds G. Ingleman, A. De Roeck \& R. Klanner, Vol I, p13.

82. V. Blobel, Proc. 1984 CERN School of Computing, Aiguablava, Spain, CERN 85-09, 88, (1985).

83. G. D'Agostini, Nucl. Instrum. Methods A 362, 487 (1995).

84. G. Zech, DESY preprint DESY 95-113, (1995).

85. A. Quadt, Oxford Thesis 1997.

86. I.G. Bird, NMC Internal Report NMC/91/1, (1991).

87. D.E. Soper \& J.C. Collins, Issues in the determination of PDFs, CTEQ Note 94/01, hep-ph/9411214;

M. Botje, M. Klein \& C. Pascaud, Proc. of the Workshop on Future Physics at HERA, DESY 1996, eds G. Ingleman, A. De Roeck \& R. Klanner, Vol I, p33;

P. Burrows et al, Proc. of Snowmass Workshop 1996, hep-ex/9612012.

88. T. Sloan, K. Bazizi, S.J. Wimpenny, Proc. ICHEP90, Singapore, edited by K.K.Phua and Y. Yamaguchi, World Scientific 1990, p639.

89. L.W. Whitlow et al, Phys. Lett. B 282, 475 (1992).

90. L.W. Whitlow, Ph.D. Thesis Stanford University, SLAC Report 357 (1990).

91. A Benvenuti et al, Phys. Lett. B 223, 485 (1989), ibid 237, 592 (1990).

92. BCDMS, A.C. Benvenuti et al, Phys. Lett. B 195, 91 (1987);

A. Ouraou, Thèse, Université Paris VII (1988).

93. NMC, M. Arneodo et al, Phys. Lett. B 364, 107 (1995).

94. NMC, E.-M. Kabuss, Proc. of DIS96, Rome, Eds G. D'Agostini \& A. Nigro, World Scientific 1997, p.136.

95. NMC, M. Arneodo et al, Nucl. Phys. B 483, 3 (1997).

96. A. Milsztajn et al, Z. Phys. C 49, 527 (1991).

97. E665, M.R. Adams et al, Phys. Rev. D 54, 3006 (1996).

98. H1, I. Abt et al, Nucl. Phys. B 407, 515 (1993);

ZEUS, M. Derrick et al, Phys. Lett. B 316, 412 (1993).

99. H1, T. Ahmed et al, Nucl. Phys. B 439, 471 (1995);

ZEUS, M. Derrick et al, Z. Phys. C 65, 379 (1995).

100. ZEUS, M. Derrick et al, Z. Phys. C 69, 607 (1996).

101. H1, C. Adloff et al, Phys. Lett. B 393, 452 (1997).

102. ZEUS, J. Breitweg et al, DESY preprint DESY 97-135.

103. ZEUS, B. Surrow, Proc. of DIS97, Chicago, http://www.hep.anl.gov/dis97/.

104. CCFR, P.Z. Quintas et al, Phys. Rev. Lett. 71, 1307 (1993).

105. CCFR, W.C. Leung et al, Phys. Lett. B 317, 655 (1993);

D. Harris et al, Fermilab-Conf-95-114.

106. CCFR, W. G. Seligman et al, hep-ex/9701017, PRL 79, 1213 (1997);

W. G. Seligman, Ph. D. thesis, Columbia University 1997, Nevis Report 292.

107. A. De Rújula et al, Nucl. Phys. B 154, 394 (1979).

108. CCFR, A. O. Bazarko et al, Z. Phys. C 65, 189 (1995).

109. S. Brodsky \& B. Ma, Phys. Lett. B 381, 317 (1996).

110. S.P. Mishra, Proc. of Lepton-Photon Symposium and Europhysics Conf, Geneva 1991, edited by S. Hegarty, K. Potter and E. Quercigh, p135.

111. M. Gluck et al, Phys. Lett. B 398, 381 (1997).

112. A. Caldwell, Unpublished talk at the 1997 DESY Theory Workshop, Hamburg, September 1997.

113. IHEP-JINR, L. S. Barabash et al, hep-ex/9611012, submitted to Phys. Lett. B.

114. L.N. Hand Phys Rev 129 (1963) 1834.

115. F.J.Gilman, Phys Rev 167 (1968) 1366.

116. EMC, J.J. Aubert et al, Nucl. Phys. B 259, 189 (1985); ibid 293, 740 (1987).

117. CDHSW, P. Berge et al, Z. Phys. C 49, 187 (1991). 
118. E140X, L.H. Tao et al, Z. Phys. C 70, 387 (1996).

119. CCFR, U.K. Yang et al, J. Phys. G. 22, 775 (1996);

A. Bodek, Proc. of DIS96, Rome Eds G. D'Agostini \& A. Nigro,

World Scientific 1997, p213.

120. NMC, A.J. Milsztajn, ibid, p220.

121. A. Bodek, S. Rock \& U.K. Yang, Univ. Rochester preprint UR-1355, 1995.

122. E. Zijlstra \& W.L. van Neerven, Nucl. Phys. B 383, 525 (1992).

123. H. Navelet et al., Phys. Lett. B 364, 357 (1996).

124. A. De Roeck and E. De Wolf, Phys. Lett. B 388, 843 (1996).

125. R.S. Thorne, hep-ph/9708302.

126. M.W. Krasny, W. Placzek \& H. Spieseberger, Z. Phys. C 53, 687 (1992);

W. Placzek, Ph.D. Thesis, Acta Physica Polonica B24, 1229 (1993).

127. A. Frey, Ph.D. Thesis, University of Bonn, BONN-IR-96-03 (1996).

128. L. Favart et al, Z. Phys. C 72, 425 (1996).

129. L. Bauerdick, A Glazov and M. Klein, Proc. of the Workshop on Future Physics at HERA, DESY 1996, editors G. Ingleman, A. De Roeck and R. Klanner, Vol I, p77.

130. BFP, A.R. Clark et al, Phys. Rev. Lett. 45, 1465 (1980);

G.D. Gollin et al, Phys. Rev. D 24, 55 (1981).

131. EMC, J.J. Aubert et al, Nucl. Phys. B 213, 31 (1983).

132. S. Riemersma, J. Smith \& W.L. van Neerven, Phys. Lett. B 347, 143 (1995).

133. B.W. Harris \& J. Smith, Nucl. Phys. B 452, 109 (1995);

Phys. Lett. B 353, 535 (1995).

134. Particle Data Group, R. M. Barnett et al, Phys. Rev. D 54, 1 (1996).

135. H1, C. Adloff et al, Z. Phys. C 72, 593 (1996).

136. G. Ingelman, J. Rathsman \& G.A. Schuler, AROMA2.1 DESY ISSN 0418-9833 (1995).

137. ZEUS, M. Derrick et al, DESY-97-089, Phys. Lett. B 407, 402 (1997).

138. NMC, M. Arneodo et al, Nucl. Phys. B 487, 3 (1997).

139. E665, M. R. Adams et al, Phys. Rev. Lett. 75, 1466 (1995).

140. NMC, M. Arneodo et al, Phys. Rev. D 50, R1 (1994).

141. R.K. Ellis, W.J. Stirling \& B.R. Webber, QCD and Collider Physics, CUP 1996.

142. A. Buras and K. Gaemers, Nucl. Phys. B 132, 249 (1978).

143. A. Buras and Bialas, Phys. Rev. D 21, 1825 (1980).

144. J.F. Owens and K. Reya, Phys. Rev. D 17, 3003 (1979).

145. C. Lopez and F.J. Yndurain, Nucl. Phys. B 171, 231 (1980), Nucl. Phys. B 183, 157 (1981); A. Gonzalez-Arroyo, et al, Nucl. Phys. B 153, 161 (1979), Nucl. Phys. B 174, 474 (1980).

146. H. Plothow-Besch, Comp.Phys.Comm 75, 396 (1993).

147. M. Virchaux and A. Milsztajn, Phys. Lett. B 274, 221 (1992).

148. P.Amaudruz et al, Nucl. Phys. B 371, 3 (1992).

149. J.J. Aubert etal, Nucl. Phys. B 293, 740 (1987), Nucl. Phys. B 213, 31 (1983).

150. K. Varvell et al, Z. Phys. C 36, 1 (1987).

151. A.V. Sidorov, Phys. Lett. B 389, 379 (1996).

152. A.L.Kataev et al, hep-ph/9706534.

153. A.V. Sidorov, hep-ph/9609345, JINR Rapid Comm.80,11 (1996).

154. J. Gomez et al, PRD 49, 4348 (1994):

R.G. Arnold et al, Phys. Rev. Lett. 52, 727 (1984);

M. Arneodo et al, Nucl. Phys. B 331, 1 (1990);

J. Ashman et al, Z. Phys. C 57, 211 (1993);

M. Arneodo et al, Nucl. Phys. B 481, 3 (1997), Nucl. Phys. B 481, 23 (1997).

155. R. Brock, PRL 44, 1027 (1980), Proc. of Physics at Fermilab in the 1990's, Breckenridge, Colorado, ed. D. Green and H.Lubatti, World Scientific 1990, p358; 
J. Kaplan and F. Martin, Nucl. Phys. B 115, 333 (1976);

H. Georgi and H.D. Politzer, Phys. Rev. D 14, 1829 (1976).

156. P.N. Harriman et al., Phys. Rev. D 42, 798 (1990).

157. J.F.Owens and W.K.Tung, Ann. Rev. Nucl. Sci. 42, 291 (1992).

158. J. Kwiecinski et al., Phys. Rev. D 42, 3645 (1990).

159. J.G. Morfin and W.K.Tung, Z. Phys. C 52, 13 (1991).

160. A.D. Martin, R.G.Roberts and W.J. Stirling, Phys. Lett. B 30, 145 (1993).

161. A.D. Martin, R.G. Roberts and W.J. Stirling, Phys. Lett. B 354, 155 (1995).

162. J. Botts et al, Phys. Lett. B 304, 159 (1995).

163. H. Lai et al, Phys. Rev. D 51, 4763 (1995).

164. H. Lai et al, Phys. Rev. D 55, 1280 (1997).

165. M.A. Parker et al, Nucl. Phys. B 232, 1 (1984).

166. D. Allasia et al, Nucl. Phys. B 239, 301 (1984), Phys. Lett. B 135, 231 (1984), Z. Phys. C 28, 321 (1985).

167. R. Field and R.P.Feynman, Phys. Rev. D 15, 2590 (1977).

168. S.A. Gurvitz, nucl-th/9701014.

169. A.W. Thomas and W. Melnitchouk, hep-ph/9708484.

170. P. Amaudruz et al, Phys. Lett. B 295, 159 (1992), Phys. Rev. Lett. 66, 2712 (1991).

171. F.M. Steffens and A.W. Thomas, Phys. Rev. C 55, 900 (1997).

172. S. Kumano, hep-ph/9707524.

173. F. Buccella et al, hep-ph/9705475.

174. H. Abramowicz et al, Z. Phys. C 15, 19 (1982).

175. H. Georgi and H.D. Politzer, Phys. Rev. D 14, 1829 (1976).

176. G. Moreno et al, Phys. Rev. D 43, 2815 (1991);

D. Malde et al, Phys. Rev. Lett. 64, 2479 (1990);

C.N. Brown et al, Phys. Rev. Lett. 63, 2537 (1989).

177. F. Abe et al,Phys. Rev. D 49, 1 (1994).

178. A. Baldit et al, Phys. Lett. B 332, 244 (1994).

179. R. Towell, Proc. of DIS97, Chicago, http://www.hep.anl.gov/dis97/.

180. F. Abe et al, Phys. Rev. Lett. 74, 850 (1995), Phys. Rev. Lett. 69, 28 (1992).

181. NMC, M. Arneodo et al., Phys. Lett. B 309, 222 (1993).

182. K. Prytz, Phys. Lett. B 311, 286 (1993);

K. Prytz, Rutherford-Appleton Laboratory preprint : RAL-94-036.

183. H1, I. Abt et al., Phys. Lett. Phys. Lett. B 321, 161 (1994).

184. K. Golec-Biernat, Phys. Lett. B 328, 495 (1994).

185. R.K. Ellis, Z. Kunst and E.M. Levin, Nucl. Phys. B 420, 517 (1994).

186. K. Bora nd D.K.Choudury, Phys. Lett. B 354, 151 (1995);

A.V.Kotikov and G.Parente, Phys. Lett. B 379, 195 (1996);

M.B. Gay-Ducati and V.P.B. Goncalves, Phys. Lett. B 390, 401 (1997).

187. E.A. Kuraev, L.N. Lipatov, V. Fadin, Soviet Phys. JETP 45, 199 (1977);

Ya.Ya. Balitsky and L.N. Lipatov, Soviet J. Nucl. Phys. 28, 822 (1978);

L.N. Lipatov, Soviet J. Nucl. Phys. 63, 904 (1980);

L.N. Lipatov,Perturbative QCD, editor A. Mueller, (World Scientific, 1989), p411.

188. J. Kwiecinski, Z. Phys. C 29, 561 (1985).

189. H1, S. Aid et al., Nucl. Phys. B 470, 3 (1996).

190. ZEUS, paper N-647, submitted to EPS HEP97 Conference, Jerusalem, August 1997.

191. S. Alekhin, IHEP 96-79, hep-ph/9611213.

192. P. Aurenche et al., Nucl. Phys. B 399, 34 (1993).

193. M. Bonesini et al, Z. Phys. C 38, 371 (1988).

194. A. Bernasconi et al, Phys. Lett. B 206, 163 (1988); 
G. Sozzi et al,Phys. Lett. B 317, 243 (1993).

195. G. Alversen et al, Phys. Rev. D 48, 5 (1993);

M. Zielinski, Proc. of DIS97, Chicago,

http://www .hep.anl.gov/dis97/, hep-ex/9711017.

196. T. Akesson et al, Soviet J. Nucl. Phys. 51, 836 (1990).

197. J. Alitti et al, Phys. Lett. B 299, 174 (1993).

198. F. Abe et al, Phys. Rev. Lett. 73, 2662 (1994).

199. H. Baer and D. Reno, Proc. of DIS97, Chicago;

R.Blair, Proc. of DIS97, Chicago, http://www.hep.anl.gov/dis97/.

200. M. Zielinski, Proc. of DIS97, Chicago, http://www.hep.anl.gov/dis97/.

201. J. Huston et al., Phys. Rev. D 51, 6139 (1995).

202. R.G. Roberts, J. Phys. G. 22, 675 (1996).

203. M. Albrow et al, hep-ph/9706470, Summary of Structure Function Subgroup, Proc. of Snowmass Workshop 1996.

204. J. Blümlein et al, Summary of Working Group I, Proc. of DIS97, Chicago, hep-ph/9707420.

205. F Abe et al, Phys. Rev. Lett. 77, 438 (1996)

F. Chlebana, Proc. of DIS97, Chicago, http://www.hep.anl.gov/dis97/

206. G.C. Blazey, Proc. of XXXI Rencontre de Moriond, March 1996, p155;

R. Hirosky, Proc. of DIS97, Chicago, http://www.hep.anl.gov/dis97/.

207. V. Barger et al, Phys. Lett. B 382, 178 (1996);

G. Altarelli etal, Phys. Lett. B 375, 292 (1996);

P. Chiappetta et al, Phys. Rev. D 54, 789 (1996);

J. Ellis and D. Ross, Phys. Lett. B 383, 187 (1996);

R.S. Chivukula et al, Phys. Lett. B 380, 92 (1996);

M. Bander, Phys. Rev. Lett. 77, 601 (1996).

208. J. Huston, Phys. Rev. Lett. 77, 444 (1996).

209. M. Klasen and G. Kramer, Phys. Lett. B 386, 384 (1996).

210. W. Giele et al, Phys. Rev. D 53, 120 (1996).

211. H1, S. Aid et al., Nucl. Phys. B 354, 494 (1995).

212. E.A.DeWolf et al, Summary of Hadron Final States Working Group, Proc. of DIS97, Chicago, http://www.hep.anl.gov/dis97/.

213. G. Frixione and G. Ridolfi, hep-ph/9707345.

214. D. Graudenz et al., Z. Phys. C 70, 77 (1996).

215. E.Zijlstra and W.L.vanNeerven, Nucl. Phys. B 383, 525 (1992);

S. Larin and J. Vermaseren, Z. Phys. C 57, 93 (1993)

216. E. Witten, Nucl. Phys. B 104, 445 (1976);

L. Orr and W.J.Stirling, Phys. Rev. Lett. 66, 1673 (1991);

E. Laenen et al, Phys. Lett. B 291, 325 (1992), Nucl. Phys. B 392, 162 (1993), Phys. Lett. B 392, 229 (1993).

217. M. Kramer et al, Phys. Lett. B 348, 657 (1995), Nucl. Phys. B 459, 3 (1996).

218. M.G.Ryskin, Z. Phys. C 57, 89 (1993);

M.G. Ryskin et al, Z. Phys. C 76, 231 (1997);

S.J.Brodsky et al, Phys. Rev. D 50, 3134 (1994).

219. R.D. Ball and S. Forte, Phys. Lett. B 335, 77 (1994), and Phys. Lett. B 336, 77 (1994).

220. M. Gluck, E. Reya and A. Vogt, Phys. Lett. B 306, 391 (1993), Nucl. Phys. B 130, 76 (1992), Z. Phys. C 53, 433 (1995).

221. A.D. Martin, R.g.Roberts and W.J.Stirling, Phys. Rev. D 51, 4763 (1995).

222. A.D. Martin et al, Phys. Lett. B 356, 89 (1995).

223. J. Huston, Proc. of DIS97, Chicago, 
S. Kuhlmann et al, Proc. of DIS97, Chicago, http://www.hep.anl.gov/dis97/.

224. M. Botje, Proc. of DIS97, Chicago, hep-ph/9707289.

225. R.G. Roberts, Proc. of DIS97, Chicago, hep-ph/9706269.

226. S. Marti, Proc. of DIS97, Chicago, http://www.hep.anl.gov/dis97/.

227. S. Bethke, Nucl.Phys.Proc.Supp. 54A, 314 (1997).

228. W.J. Stirling, hep-ph/9709429.

229. M. Weber, Proc. of DIS97, Chicago, http://www.hep.anl.gov/dis97/.

230. D. Graudenz, hep-ph/9708362.

231. M. Botje, Proc. of DIS97, Chicago, hep-ph/9707292.

232. J. Blümlein et al, Proc. of the Workshop on Future Physics at HERA, DESY 96, eds G. Ingelman, A. De Roeck \& R. Klanner, Vol I, p52.

233. A.L. Kataev et al, Phys. Lett. B 388, 179 (1996).

234. J. Chyla and J.Rames, Z. Phys. C 31, 151 (1986): V.G. Krivokhizkhin et al, Z. Phys. C 36, 51 (1987),Z. Phys. C 48, 347 (1990);

A.V. Kotikov, G. Parente and J. Sanchez-Guillen, Z. Phys. C 58, 465 (1993);

A.L. Kataev and A.V. Sidorov, Phys. Lett. B 331, 179 (1994);

J. Chyla and J. Rames, Phys. Lett. B 343, 351 (1995)

235. H. Abramowicz et al, Phys. Rev. Lett. 57, 298 (1986), Z. Phys. C 28, 51 (1985);

J. Berge et al, Z. Phys. C 49, 187 (1991).

236. V.V. Ammsov et al, Z. Phys. C 30, 175 (1986).

237. P.C. Bosetti et al, Nucl. Phys. B 203, 362 (1982).

238. L.S. Barabash et al., JINR-E1-96-308, hep-ex/9611012.

239. A.L. Kataev, hep-ph/9709509.

240. M.V. Tokarev and A.V.Sidorov, hep-ph/9707438.

241. D.V. Shirkov et al, hep-ph/9707514.

242. M. Shaevitz et al, Nucl. Phys. Proc. Supp. B38, 188 (1995).

243. J. Chyla and A. L. Kataev,Phys. Lett. B 297, 385 (1992).

244. P. Spentzouris, Proc. of DIS97, Chicago, http://www.hep.anl.gov/dis97/.

245. R.D. Ball and S. Forte, Phys. Lett. B 358, 365 (1995).

246. A. De Roeck et al, Phys. Lett. B 385, 411 (1996).

247. R.D. Ball and S. Forte, Proc. of DIS96, Rome, Eds G. D'Agostini \& A. Nigro, World Scientific 1997, p208.

248. R.S. Thorne, Phys. Lett. B 392, 463 (1997), hep-ph/9701241, hep-ph/9710541, Proc. of DIS97, Chicago, hep-ph/9706233.

249. R.S. Thorne, private communication.

250. (a) F. Buccella \& J. Soffer, Mod. Phys. Lett. A8, 225 (1993);

(b) C. Bourrely et al, Z. Phys. C 62, 431 (1994);

(c) C. Bourrely \& J. Soffer, Phys. Rev. D 51, 2108 (1995);

(d) C. Bourrely \& J. Soffer, Nucl. Phys. B 445, 341 (1995).

251. C. Bourrely \& J. Soffer, Phys. Rev. D 53, 4067 (1996); F. Buccella et al, Proc. of HADRONS96, Ukraine, p130; G. Miele, Cont. to Lepton-Photon Symposium, Beijing 1995, hep-ph/9508203; F. Buccella et al, hep-ph/9705475.

252. J. Ellis and R.L. Jaffe, Phys. Rev. D 9, 1444 (1974), Phys. Rev. D 10, 1669 (1974).

253. G. Mangano, G. Miele \& G. Migliore, Nuovo Cim. A 108, 867 (1995).

254. R. S. Bhalerao, Phys. Lett. B 380, 1 (1996); erratum ibid B387, 881 (1996).

255. A. Chodos et al, Phys. Rev. D 10, 2599 (1974).

256. F.M. Steffens \& A.W. Thomas, Nucl. Phys. A 568, 798 (1994).

257. F.M. Steffens \& A.W. Thomas, Prog. Th. Phys. Suppl. 120, 145 (1995).

258. J. Jasiak, hep-ph/9706503.

259. M. Göckeler et al, Phys. Rev. D 53, 2317 (1996).

260. C. Best et al, Minireview, Proc. of DIS97, Chicago, hep-ph/9706502. 
261. L. Mankiewicz \& T. Weigl, Phys. Lett. B 380, 134 (1996);

Phys. Lett. B 389, 334 (1996).

262. B.L. Ioffe, V.A. Khoze \& L.N. Lipatov, Hard Processes, North-Holland (1984), Ch. 3.

263. J.D. Bjorken, Talk at the SLAC Summer Institute 1996, hep-ph/9611421.

264. T. Schäfer \& E.V. Shuryak, hep-ph/9610451.

265. D.I. Diakonov et al, Nucl. Phys. B 480, 341 (1996); Phys. Rev. D 56, 4069 (1997).

266. M. Glück, E. Reya, M. Stratmann \& W. Vogelsang, Phys. Rev. D 53, 4775 (1996).

267. L. Gamberg, H. Reinhardt \& H. Weigl, talk at the MENU97 Conference, Vancouver, July 1997, hep-ph/9708266.

268. P. Hoyer \& D.P. Roy, hep-ph/9705273

269. S.J. Brodsky et al, Phys. Lett. B 93, 451 (1980); Phys. Rev. D 23, 2745 (1981).

270. A. de Rujula et al., Phys. Rev. D 10, 1649 (1974).

271. H. Navelet et al, Mod. Phys. Lett. A 12, 857 (1997).

272. F.J. Yndurain, hep-ph/9604263, hep-ph/9605265.

273. J. Kwiecinski, J. Phys. G. 19, 1443 (1993).

274. V. Fadin and L.N. Lipatov, Nucl. Phys. B 477, 767 (1996);

V.S. Fadin, R.Fiore and A.Quartarolo, Phys. Rev. D 53, 2729 (1996);

V.S. Fadin, R.Fiore and M.I.Kotsky, Phys. Lett. B 359, 181 (1995), Phys. Lett. B 389, 737 (1996), Phys. Lett. B 387, 593 (1996), hep-ph/9704267;

V.Del Duca, Phys. Rev. D 54, 989 (1996), Phys. Rev. D 54, 4474 (1996);

G. Camici and M. Ciafaloni, Phys. Lett. B 386, 341 (1996), Nucl. Phys. B 496, 305 (1997), hep-ph/9707390;

A.Stepanova and F.V. Tkachov, hep-ph/9710242;

V.S. Fadin et al, hep-ph/9711427.

275. Y.L. Dokshitzer, Summary talk, Proc. of DIS97, Chicago, hep-ph/9706375.

276. E.M. Levin, Summary talk, Proc. of DIS97, Chicago, hep-ph/9706341, hep-ph/9706448.

277. M. Ciafaloni, Nucl. Phys. B 296, 49 (1988);

S. Catani, F. Fiorini and G. Marchesini, Phys. Lett. B 234, 339 (1990); Nucl. Phys. B 336, 18 (1990).

278. G. Marchesini and B.R. Webber, Nucl. Phys. B 349, 617 (1991);

G. Marchesini, Proc. of 1990 Workshop on QCD at $200 \mathrm{TeV}$, edited by L. Cifarelli and Yu. Dokshitzer,(N.Y.Plenum, 1990)p.183

279. J. Kwiecinski, A.D. Martin and P.J. Sutton, Phys. Rev. D 52, 1445 (1995), Phys. Rev. D 53, 6094 (1996).

280. J. Kwiecinski, A.D. Martin and P.J. Sutton, Z. Phys. C 71, 585 (1996);

B. Andersson, G. Gustafson and J. Samuleson, Nucl. Phys. B 463, 217 (1996);

M. Ciafaloni, Nucl. Phys. B 296, 49 (1988).

281. G. Bottazzi et al, hep-ph/9702418; hep-ph/9708474;

G. Salam, Proc. of DIS97, Chicago, hep-ph/9705233; hep-ph/9707383;

M. Scorletti, hep-ph/9710559.

282. H. Li, hep-ph/9703328.

283. H. Li, hep-ph/9709236.

284. J. Kwiecinski, A.D. Martin and A.M. Stasto, Phys. Rev. D 56, 3991 (1997).

285. A.L.Ayala et al, Phys. Lett. B 388, 188 (1996);

S.M.Troshin and N.E.Tyurin, Europhys.Lett. 37, 239 (1997).

286. A.H. Mueller, J. Phys. G. 19, 1463 (1993),

Nucl. Phys. B (Proc. Suppl.) 18C (1990) 125.

287. M.G. Ryskin and E.M. Levin, Phys.Rep. 189, 267 (1990);

A.H. Mueller, Nucl. Phys. B 335, 115 (1990);

W.Zhu et al, Phys. Rev. D 54, 847 (1996). 
288. A.H. Mueller, Phys. Lett. B 396, 251 (1997).

289. F.M. Lev, hep-ph/9606204, hep-ph/9606334.

290. L.V. Gribov, E.M. Levin, M.G. Ryskin, Phys.Rep 100, 1 (1983);Nucl. Phys. B 188, 155 (1981) A.H. Mueller and J. Qiu, Nucl. Phys. B 260, 427 (1986).

291. J. Kwiecinski et al, Phys. Rev. D 44, 2640 (1991), Phys. Rev. D 46, 921 (1992); A.J. Askew et al, Phys. Rev. D 47, 3775 (1993), Nucl. Phys. B 416, 739 (1994), Phys. Rev. D 49, 4402 (1994), Phys. Lett. B 325, 212 (1994), Mod. Phys. Lett. A 8, 3813 (1993).

292. E. Gotsman, E.M. Levin and U. Maor, Phys. Lett. B 379, 186 (1996).

293. W.Zhu et al, —NPB 449, 183 (1995)

294. J. Bartels, Z. Phys. C 60, 4171 (1993), Phys. Lett. B 298, 204 (1993);

E.M. Levin,M.G. Ryskin and A.G. Shuvaev, Nucl. Phys. B 387, 519 (1992);

E. Laenen, E.M. Levin and A.G. Shuvaev, Nucl. Phys. B 419, 38 (1994);

A. Martin, J. Phys. G. 19, 1604 (1993).

295. E. Laenen and E.M. Levin, Nucl. Phys. B 451, 207 (1995).

296. see for example, E.M. Levin, hep-ph/9710546.

297. A.L. Ayala, M. B. Gay-Ducati and E.M. Levin, Nucl. Phys. B 493, 305 (1997), hep-ph/9706347, hep-ph/9710539.

298. Yu. M. Shabelski and D.Treleani, Phys. Lett. B 403, 364 (1977).

299. J. Bartels, Phys. Lett. B 298, 204 (1993), Z. Phys. C 60, 471 (1993);

E.M. Levin, M.G. Ryskin and A .G. Shuvaev, Nucl. Phys. B 387, 580 (1992);

A.P. Bukhvostov et al, Nucl. Phys. B 258, 601 (1985).

300. J. Bartels and C. Bontus, Proc. of DIS97, Chicago, http://www.hep.anl.gov/dis97/.

301. A.H. Mueller, Nucl. Phys. B 415, 373 (1994);

A.H. Mueller and B. Patel, Nucl. Phys. B 425, 471 (1995);

A.H. Mueller, Nucl. Phys. B 437, 107 (1995);

Chen Zhang and A.H. Mueller, Nucl. Phys. B 451, 579 (1995)

302. R. Peschanski and G. Salam, Proc. of the Workshop on Future Physics at HERA, DESY 1996, eds G. Ingelman, A. De Roeck \& R. Klanner, Vol. I, p110.

303. J. Jalilian-Marian et al, Phys. Rev. D 55, 5414 (1997), hep-ph/9701284, hep-ph/9706377, hep-ph/9709432;

L. McLerran and R. Venugopalan, Phys. Rev. D 49, 2233,3352 (1994), Phys. Rev. D 50, 2225 (1994), Phys. Rev. D 53, 458 (1996)

304. A.R. White, Proc. of DIS97, Chicago, hep-ph/9705297;

C. Ewerz, hep-ph/9707257.

305. W. Buchmuller and D. Haidt, hep-ph/9605428.

306. D. Haidt, Proc. of DIS97, Chicago, http://www.hep.anl.gov/dis97/.

307. A. De Roeck and E. de Wolf, Phys. Lett. B 388, 843 (1996).

308. L. Mankiewicz et al, Phys. Lett. B 393, 175 (1997).

309. R.D. Ball and S. Forte, Acta Physica Polonica B26, 2097 (1995)

310. C. Lopez, F. Barreiro and F.J. Yndurain, Z. Phys. C 72, 561 (1996).

311. K. Adel, F. Barreiro and F.J.Yndurain, Nucl. Phys. B 495, 221 (1997).

312. C. Lopez and F.J. Yndurain, Phys. Rev. Lett. 44, 1118 (1980).

313. K. Adel et al, hep-ph/9612469.

314. J.R. Forshaw, P.N. Harriman and P.J. Sutton, J. Phys. G. 19, 1616 (1993).

315. M.F. McDermott, J.R. Forshaw and G.G. Ross, Phys. Lett. B 349, 189 (1995); M.F. McDermott and J.R. Forshaw, Nucl. Phys. B 484, 283 (1997).

316. G. Camici and M. Ciafaloni, hep-ph/9707390, Phys. Lett. B 395, 118 (1997).

317. J. Bartels, J. Phys. G. 19, 1611 (1993);

J. Bartels, H. Lotter and M. Vogt, Phys. Lett. B 373, 215 (1996). 
318. I. Bojak and M. Ernst, Phys. Rev. D 53, 80 (1996).

319. J.C. Collins and P.V. Landshoff, Phys. Lett. B 276, 196 (1992).

320. K.D. Anderson et al, Proc. of DIS97, Chicago, hep-ph/9706215; hep-ph/9705466.

321. G. Camici and M. Ciafaloni, Phys. Lett. B 386, 341 (1996).

322. M. Ciafaloni, hep-ph/9709390.

323. L.P.A. Haakman et al, hep-ph/9707262.

324. R.D. Ball and S. Forte, Phys. Lett. B 405, 317 (1997), Proc. of DIS97, Chicago, hep-ph/9706459.

325. A.R. White, Proc. of AUP Workshop on QCD, Paris 1996, p283.

326. Askew et al, Phys. Lett. B 325, 212 (1994).

327. I. Bojak and M.Ernst, hep-ph/9702282.

328. H. Navelet, R. Peschanski, Ch. Royon, S. Wallon, Phys. Lett. B 385, 357 (1996).

329. V.R. Zoller, Proc. of DIS97, Chicago, hep-ph/9706370;

N.N.Nikolaev et al, JETP, Lett. 66, 138 (1997).

330. M. Braun and G.P.Vacca, hep-ph/9706314.

331. S. Catani and F. Hautmann,

Nucl. Phys. B 427, 475 (1995); Phys. Lett. B 315, 517 (1993);

S. Catani. M. Ciafaloni and F. Hautmann,

Nucl. Phys. B 366, 135 (!991); Phys. Lett. B 242, 97 (1990);

J. Collins and R.K. Ellis, Nucl. Phys. B 360, 3 (1991).

332. J. Kwiecinski and A.D. Martin, Phys. Lett. B 353, 123 (1995).

333. M. Ciafaloni, Phys. Lett. B 356, 74 (1995);

S. Catani, Proc. of DIS95, Paris Eds. J.F. Laporte and Y. Sirois, p281;

R.D. Ball and S. Forte, Phys. Lett. B 358, 365 (1995), Proc. of DIS95, Paris, Eds. J.F. Laporte and Y. Sirois, p273.

334. H. Li, Phys. Lett. B 405, 347 (1997).

335. G. Camici and M. Ciafaloni, Nucl. Phys. B 496, 305 (1997).

336. S. Catani, Z. Phys. C 70, 263 (1996).

337. R.K. Ellis, F. Hautmann and B.R. Webber, Phys. Lett. B 348, 582 (1995).

338. J. Blümlein, S. Riemersma, A.Vogt, Acta Physica Polonica B28, 211 (1997); Proc. of SPIN96, Amsterdam p218; Nucl. Phys. Proc. Supp. 51C, 30 (1996); Proc. of DIS96, Rome, Eds G. D'Agostini \& A. Nigro, World Scientific 1997, p572.

339. J. Blümlein and A. Vogt, Proc. of DIS97, Chicago, hep-ph/9706371; hep-ph/9707488.

340. R.S. Thorne, private communication.

341. R.D. Ball and S. Forte, Phys. Lett. B 359, 362 (1995).

342. S. Catani, Z. Phys. C 75, 665 (1997).

343. R.D. Ball and S. Forte, Proc. of DIS95, Paris, p273.

344. R.K. Ellis, F. Hautmann and B.R. Webber, Phys. Lett. B 348, 582 (1995);

R.D. Ball and S. Forte, Phys. Lett. B 351, 513 (1995); Proc. of XXX Recontres de Moriond, 1995, p143; Acta Physica Polonica B26, 2097 (1995); Proc. of DIS96, Rome, Eds G. D'Agostini \& A. Nigro, World Scientific 1997, p172;

J.R. Forshaw, R.G. Roberts and R.S. Thorne, Phys. Lett. B 356, 79 (1995);

R.S. Thorne, Proc. of EPS Conference, Brussels 1995, edited by J. Lemone, C. VanderVelde and F. Verbeure, p123.

345. J.R. Forshaw, R.G. Roberts and R.S. Thorne, Phys. Lett. B 356, 79 (1995).

346. I. Bojak and M. Ernst, Phys. Lett. B 397, 296 (1997).

347. R.S. Thorne, Proc. of DIS96, Rome, Eds G. D'Agostini \& A. Nigro, World Scientific 1997, p234; Proc. of the Workshop on Future Physics at HERA, DESY 1996 eds G. Ingelman, A. De Roeck \& R. Klanner, Vol I, p107; Proc. of DIS97, Chicago, hep-ph/9706233. 
348. A.D. Martin, Acta Physica Polonica B27, 1287 (1996);

M.G. Ryskin, Yu.M. Shabelskii, Phys. Atom. Nucl 58, 1782 (1995).

349. V. del Duca et al, Phys. Rev. D 51, 4756 (1995).

350. W.L. vanNeerven, Proc. of the Workshop on future Physics at HERA, DESY 1996 eds G. Ingelman, A. De Roeck \& R. Klanner, Vol I, p56.

351. R.D. Ball and S. Forte, Phys. Lett. B 351, 313 (1995).

352. A.V. Kotikov and G. Parente, hep-ph/9609439, Proc. of DIS96, Rome, Eds G. D'Agostini \& A. Nigro, World Scientific 1997, p237; hep-ph/9710252.

353. R. Deka and D.K.Choudury, Z. Phys. C 75, 679 (1997);

T. Sarkar and R. Basu, hep-ph/9607232.

354. A.H. Mueller, Columbia preprint CU-TP-658 (1994).

355. J. Bartels, H. Lotter, Phys. Rev. Lett. 309, 400 (1993).

356. J. Kwiecinski et al, Phys. Rev. D 50, 217 (1994); K. Golec-Biernat et al, Phys. Lett. B 335, 220 (1994).

357. A.H. Mueller, Nucl. Phys. B (Proc. Suppl.) 18C (1990) 125;

J. Phys. G17 (1991) 1443;

A.H. Mueller and H. Navelet, Nucl. Phys. B 282, 727 (1987);

V. del Duca and C. R, Schmidt, Phys. Rev. D 49, 4510 (1994);

W.J.Stirling, Nucl. Phys. B 423, 56 (1994);

V. del Duca, hep-ph/9707348;

J. Bartels et al, Z. Phys. C 76, 75 (1997).

358. J. Bartels, A. De Roeck and M. Loewe, Z. Phys. C 54, 635 (1992);

J. Kwiecinski, A.D. Martin and P.J. Sutton, Phys. Lett. B 287, 254 (1992),

Phys. Rev. D 46, 921 (1992);

W.K. Tang, Phys. Lett. B 278, 363 (1991).

359. A.J. Askew et al, Phys. Lett. B 338, 92 (1994);

J. Kwiecinski et al., Phys. Rev. D 54, 1874 (1996);

J. Kwiecinski et al., hep-ph/9707375.

360. V.T.Kim and G.B. Pivovarov, hep-ph/9709433.

361. J. Bartels et al, Phys. Lett. B 384, 300 (1996).

362. B. Andersson et al, Phys.Rep. 97, 31 (1983);

T. Sjöstrand, Comp. Phys. Comm. 39 (1986) 347;

T. Sjöstrand and M. Bengtsson, Comp. Phys. Comm. 43 (1987) 367;

T. Sjöstrand, CERN-TH-6488-92 (1992).

363. G. Marchesini et al., Comp. Phys. Comm. 67 (1992) 46;

HERWIG MC Version 5.9, G. Marchesini et al, hep-ph/9607393.

364. C.R. Schmidt, Phys. Rev. Lett. 78, 4531 (1997).

365. L.H. Orr and W.J. Stirling, hep-ph/9706529.

366. H. Kharraziha and L. Lönnblad, hep-ph/9709424;

B. Anersson et al, hep-ph/9711403

367. H1, S. Aid et al., Phys. Lett. B 356, 118 (1995).

368. G. Ingelman, A. Edin, J. Rathsman, Comp. Phys. Comm. 101 (1997) 108;

A. Edin et al, J. Phys. G. 22, 943 (1996).

369. L. Lönnblad, J. Phys. G. 22, 947 (1996).

370. A. Edin, G. Ingelman and J. Rathsman, Z. Phys. C 75, 57 (1997), Phys. Lett. B 336, 371 (1996),

371. H1, C. Adloff et al., paper pa02-73, submitted to ICHEP 96, Warsaw, July 1996.

372. N. A. Pavel, Proc. of DIS96, Rome, Ed.s G. D'Agostini, A. Nigro, (1997) 502.

373. M. Kuhlen Phys. Lett. B 382, 441 (1996).

374. H1, C. Adloff et al., Nucl. Phys. B 485, 3 (1997).

375. J. Kwiecinski, S.C. Lang, A.D. Martin, DTP-97-56, hep-ph/9707240. 
376. A.H. Mueller, J. Phys. G. 17, 1443 (1991);

J. Bartels et al, Z. Phys. C 54, 635 (1992);

W.K. Tung, Phys. Lett. B 278, 363 (1992);

J. Kwiecinski et al, Phys. Rev. D 46, 921 (1992), Phys. Lett. B 287, 254 (1992).

377. H1, paper pa03-049, submitted to ICHEP 96, Warsaw, Poland, July 1996.

378. ZEUS, paper N-659, submitted to EPS/HEP Conference 97, Jerusalem, August 1997.

379. J. Kwiecinski et al, Phys. Rev. D 54, 1874 (1996), Phys. Rev. D 55, 1273 (1997).

380. J. Kwiecinski et al, Acta Physica Polonica B27, 3455 (1996).

381. J. Bartels, A. De Roeck and M. Wüsthoff, Proc. of the Workshop on Future Physics at HERA, DESY 1996, Eds. G. Ingelman,.A. De Roeck, R. Klanner, p598.

382. E. Mirkes and D. Zeppenfeld, Acta Physica Polonica B27, 1323 (1996), Phys. Rev. Lett. 78, 428 (1997), Proc. of the Workshop on Future Physics at HERA, DESY 1996, eds G. Ingelman, A. De Roeck \& R. Klanner, p588, hep-ph/9706437, hep-ph/9711224.

383. S. Catani and M.H. Seymour, Phys. Lett. B 378, 287 (1996), Nucl. Phys. B 485, 291 (1997), Phys. Rev. D 55, 6189 (1997).

384. D. Graudenz, hep-ph/9710244.

385. H. Jung, hep-ph/9709425.

386. J. Bartels, A. De Roeck, H. Lotter, Phys. Lett. B 389, 742 (1996).

387. S.J. Brodsky, F. Hautmann, D.E. Soper, Phys. Rev. Lett. 78, 803 (1997).

388. J.R. Cudell et al, hep-ph/9601336, hep-ph/9701312.

389. A. Donnachie and P.V. Landshoff, Z. Phys. C 61, 139 (1994).

390. T. Aid et al, Z. Phys. C 69, 27 (1995).

391. M. Derrick et al, Z. Phys. C 63, 408 (1995).

392. A. Levy, Proc. of DIS96, Rome Eds. A. Negri and G. D'Agostini, and Tel-Aviv preprint TAUP-2349-96 (1996);

A. Levy, DESY preprint DESY 97-013 (1997).

393. B. Badelek, J. Kwiecinski, Rev. Mod. Phys. 68 (1996) 445.

394. H. Abramowicz, E.M. Levin, A. Levy and U. Maor, Phys. Lett. B 269, 465 (1991). We show the parametrizations as presented in: A. Marcus PhD. thesis Tel-Aviv University TAUP 2350-96 (1996) (unpublished).

395. A. Capella et al., Phys. Lett. B 337, 358 (1994); E. Gotsman, E.M. Levin and U. Maor, Phys. Lett. B 379, 186 (1996); J.R.Cudell, P.V. Landshoff and A. Donnachie, Nucl. Phys. B 482, 241 (1996).

396. E.M. Levin, CBPF-NF-010/95, Lectures to the Gleb Wataghiin School, Campinas, Brazil, 1994, p158.

397. B. Badelek and J. Kwiecinski, Phys. Lett. B 295, 263 (1992), Z. Phys. C 43, 251 (1989).

398. D. Schildknecht and H. Spiesberger Bielefeld Uni. preprint BI-TP-97-25 (1997).

399. J.D. Sakurai and D. Schildknecht, Phys. Lett. B 40, 121 (1972);

B. Gorczyca and D. Schildknecht, Phys. Lett. B 47, 71 (1973).

400. G. Kerley and G. Shaw, Manchester University preprint MC-TH-97-12 (1997).

401. S.M. Troshin and N.E.Tyurin, Phys. Rev. D 55, 7305 (1997).

402. A.V. Kotikov, hep-ph/9507320.

403. E. Gotsman, E.M. Levin and U. Maor, hep-ph/9708275.

404. V. Shekelyan, to be published in Proc. of the $199^{7}$ Symposium on Lepton and Photon Interactions, Hamburg, July 1997, eds. A. De Roeck and A. Wagner.

405. A. D. Martin, private communication and Nucl. Phys. B 392, 162 (1993).

406. D. Acosta, U. Bassler, invited talks at EPS HEP97 Conference, Jerusalem, August 1997, http://www. cern. ch/hep97/. 
407. H1, C. Adloff et al, Z. Phys. C 74, 191 (1997).

408. ZEUS, J. Breitweg et al, Z. Phys. C 74, 207 (1997).

409. Particle Data Group, R.M. Barnett et al, Phys. Rev. D 54, 1 (1996).

410. C. Pascaud \& F. Zomer, preprint LAL 95-05 (1995);

M. Botje, M. Klein \& C. Pascaud, Proc. of the Workshop on Future Physics at HERA, DESY 1996, eds G. Ingelman, A. De Roeck \& R. Klanner, Vol.1 p33 (1996); M. Botje, Proc. of DIS97, Chicago, hep-ph/9707289.

411. G. Wolf, DESY preprint DESY 97-047, hep-ex/9704006.

412. U. Bassler \& G. Bernardi, DESY preprint DESY 97-136.

413. S. Kuhlmann, H. L. Lai \& W. K. Tung, Phys. Lett. B 409, 271 (1997).

414. S. Rock \& P. Bosted, hep-ph/9706436.

415. E.D. Bloom \& F.J. Gilman, Phys. Rev. Lett. 25, 1140 (1970).

416. J.F. Gunion \& R. Vogt, hep-ph/9706252.

417. W. Melnitchouk \& A.W. Thomas, hep-ph/9707387.

418. A. Szczurek and A. Budzabowski, Phys. Lett. B 408, 275 (1997).

419. N.I. Kochelev, hep-ph/9710540.

420. G. Altarelli et al, CERN preprint CERN-TH/97-40, hep-ph/9703276.

421. B. Straub, to be published in Proc. of the 1997 Symposium on Lepton Photon Interactions, Hamburg, July 1997, eds. A. De Roeck and A. Wagner, http: //www.desy.de/lp97/.

422. E. Elsen, to be published in Proc. of EPS/HEP Conference 97, Jerusalem, August 1997, http: //www. cern. ch/hep97/.

423. Proc. of the Workshop on Future Physics at HERA, DESY 1996, eds G. Ingelman, A. De Roeck \& R. Klanner, 2 Vols. DESY 1996. 